\title{
A Practical Solution to Stereodefined Tetrasubstituted Olefins
}

Jianxin Dai, Minyan Wang, Guobi Chai,

Chunling $\mathrm{Fu}$, and Shengming $\mathrm{Ma}^{*}$

Laboratory of Molecular Recognition and Synthesis, Department of Chemistry,

Zhejiang University, Hangzhou 310027, People's Republic of China

E-mail:masm@sioc.ac.cn; Fax: (+86)21-64167510

Table of Contents

\begin{tabular}{|l|c|}
\hline General information & $\mathrm{S} 2$ \\
\hline Experimental details and analytical data & $\mathrm{S} 2-\mathrm{S} 35$ \\
\hline References & $\mathrm{S} 36$ \\
\hline${ }^{1} \mathrm{H}$ NMR, ${ }^{13} \mathrm{C}$ NMR, and NOESY Spectra & S37-S144 \\
\hline
\end{tabular}


General Information: ${ }^{1} \mathrm{H}$ NMR and ${ }^{13} \mathrm{C}$ NMR spectra were recorded in $\mathrm{CDCl}_{3}$ using a $300 \mathrm{MHz}$ NMR spectrometer $\left({ }^{1} \mathrm{H}\right.$ at $300 \mathrm{MHz}$ and ${ }^{13} \mathrm{C}$ at $\left.75 \mathrm{MHz}\right)$. Mass spectra (MS) and high-resolution mass spectra (HRMS) were carried out using electron impact ionization (EI) or electron spray ionization (ESI). 2,3-Allenols were prepared using the reduction reaction of 2,3-allenoates with DIBAL-H according to the literature. ${ }^{1}$ 2,3-Allenals were prepared using the iron-catalyzed aerobic oxidation of 2,3-allenols according to the literature. ${ }^{2} \mathrm{Et}_{2} \mathrm{Zn}$ (1.5 $\mathrm{M}$ in toluene) was purchased from Acros. $\mathrm{Et}_{2} \mathrm{Zn}\left(2.0 \mathrm{M}\right.$ in toluene) and $n-\mathrm{Bu}_{2} \mathrm{Zn}(1.0 \mathrm{M}$ in toluene) were purchased from Energy Chemicals. $i-\operatorname{Pr}_{2} \operatorname{Zn}(1.0 \mathrm{M}$ in toluene) was purchased from Sigma-Aldrich. $\mathrm{Ph}_{2} \mathrm{Zn}$ was purchased from Alfa Aesar and kept in a glove box. Toluene, THF, and $\mathrm{Et}_{2} \mathrm{O}$ were freshly distilled from $\mathrm{Na}$ wire/benzophenone before use. Other commercially available reagents were purchased and used without further purification.

\section{Experimental details and analytical data}

\section{Synthesis of 2,3-allenals}

1. 2-Ethyltetradeca-2,3-dienal (1a) (djx-3-075)

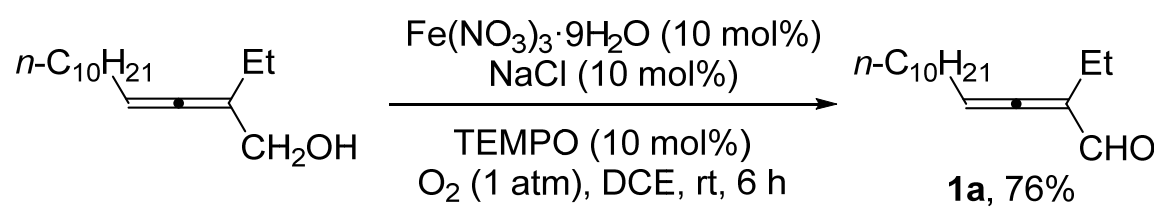

Typical Procedure I: To a dry three-neck flask were added $\mathrm{Fe}\left(\mathrm{NO}_{3}\right)_{3} \cdot 9 \mathrm{H}_{2} \mathrm{O}$ (0.5657 g, $1.4 \mathrm{mmol})$, TEMPO (0.2185 g, $1.4 \mathrm{mmol}), \mathrm{NaCl}(0.0821 \mathrm{~g}, 1.4 \mathrm{mmol})$, and DCE $(40 \mathrm{~mL})$ under an oxygen atmosphere. Then 2-ethyltetradeca-2,3-dien-1-ol (3.3319 g, $14.0 \mathrm{mmol}) / \mathrm{DCE}(10 \mathrm{~mL})$ was added with stirring at room temperature. After being stirred for $6 \mathrm{~h}$, the reaction was complete as monitored by TCL. After the solvent was removed by evaporation, the residue was purified by flash chromatography on silica gel to afford 1a $(2.5118 \mathrm{~g}, 76 \%)$ (eluent: petroleum ether/diethyl ether $=100 / 1)$ as a liquid: ${ }^{1} \mathrm{H}$ NMR $\left(300 \mathrm{MHz}, \mathrm{CDCl}_{3}\right) \delta 9.53(\mathrm{~s}, 1 \mathrm{H}$, $\mathrm{CHO}), 5.81-5.70(\mathrm{~m}, 1 \mathrm{H},=\mathrm{CH}), 2.25-2.12\left(\mathrm{~m}, 4 \mathrm{H}, \mathrm{CH}_{2} \times 2\right), 1.54-1.19\left(\mathrm{~m}, 16 \mathrm{H}, \mathrm{CH}_{2}\right.$ 
$\times 8), 1.03\left(\mathrm{t}, J=7.5 \mathrm{~Hz}, 3 \mathrm{H}, \mathrm{CH}_{3}\right), 0.88\left(\mathrm{t}, J=6.8 \mathrm{~Hz}, 3 \mathrm{H}, \mathrm{CH}_{3}\right) ;{ }^{13} \mathrm{C} \mathrm{NMR}(75 \mathrm{MHz}$, $\left.\mathrm{CDCl}_{3}\right) \delta 218.0,192.6,113.4,97.0,31.8,29.5,29.3,29.2,28.9,27.9,22.6,17.9,14.0$, 12.0; IR (neat) $v\left(\mathrm{~cm}^{-1}\right) 2958,2926,2854,2807,2756,2700,1943,1686,1459,1378$, 1209, 1177, 1062; MS (70 ev, EI) m/z (\%) $236\left(\mathrm{M}^{+}, 1.80\right), 109$ (100); HRMS calcd for $\mathrm{C}_{16} \mathrm{H}_{28} \mathrm{O}\left[\mathrm{M}^{+}\right]: 236.2140$, found: 236.2144 .

\section{2-Ethyltrideca-2,3-dienal (1b) (djx-2-191)}
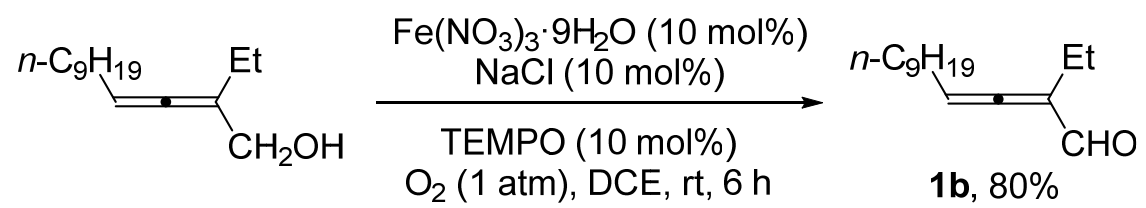

Following Typical Procedure I, the reaction of $\mathrm{Fe}\left(\mathrm{NO}_{3}\right)_{3} \cdot 9 \mathrm{H}_{2} \mathrm{O}(0.1214 \mathrm{~g}, 0.3$ mmol), TEMPO (0.0471 g, $0.3 \mathrm{mmol}), \mathrm{NaCl}(0.0178 \mathrm{~g}, 0.3 \mathrm{mmol}), \mathrm{DCE}(15 \mathrm{~mL})$, and 2-ethyltrideca-2,3-dien-1-ol (0.6720 g, $3.0 \mathrm{mmol}) / \mathrm{DCE}(5 \mathrm{~mL})$ for $6 \mathrm{~h}$ afforded 1b $(0.5327 \mathrm{~g}, 80 \%)$ (eluent: petroleum ether $\left(30-60{ }^{\circ} \mathrm{C}\right) / \mathrm{ethyl}$ acetate $\left.=100 / 1\right)$ as a liquid: ${ }^{1} \mathrm{H}$ NMR (300 MHz, $\left.\mathrm{CDCl}_{3}\right) \delta 9.53(\mathrm{~s}, 1 \mathrm{H}, \mathrm{CHO}), 5.80-5.70(\mathrm{~m}, 1 \mathrm{H}, \mathrm{C}=\mathrm{CH})$, 2.29-2.08 (m, 4H, $\left.\mathrm{CH}_{2} \times 2\right), 1.55-1.18\left(\mathrm{~m}, 14 \mathrm{H}, \mathrm{CH}_{2} \times 7\right), 1.03(\mathrm{t}, J=7.4 \mathrm{~Hz}, 3 \mathrm{H}$, $\left.\mathrm{CH}_{3}\right), 0.88\left(\mathrm{t}, J=6.9 \mathrm{~Hz}, 3 \mathrm{H}, \mathrm{CH}_{3}\right) ;{ }^{13} \mathrm{C} \mathrm{NMR}\left(75 \mathrm{MHz}, \mathrm{CDCl}_{3}\right) \delta 218.0,192.7,113.4$, 97.0, 31.8, 29.5, 29.3, 29.2, 28.9, 27.9, 22.6, 17.9, 14.0, 12.0; IR (neat) $v\left(\mathrm{~cm}^{-1}\right) 2958$, 2926, 2855, 2807, 2753, 2697, 1943, 1686, 1459, 1378, 1210, 1177, 1063; MS (70 ev, EI) $m / z(\%) 222\left(\mathrm{M}^{+}, 4.40\right), 109$ (100); HRMS calcd for $\mathrm{C}_{15} \mathrm{H}_{26} \mathrm{O}\left[\mathrm{M}^{+}\right]$: 222.1984, found: 222.1982 .

\section{2-Methyltrideca-2,3-dienal (1c) (djx-3-017)}
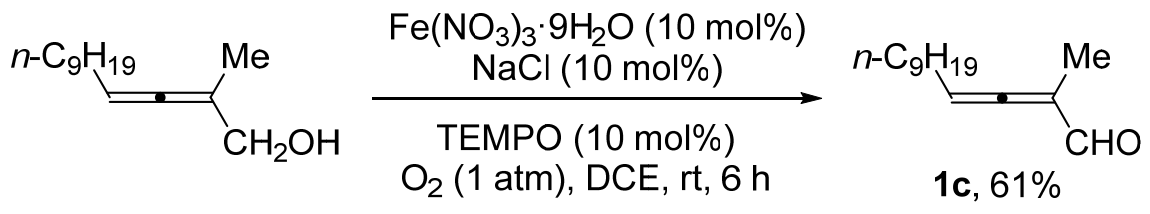

Following Typical Procedure I, the reaction of $\mathrm{Fe}\left(\mathrm{NO}_{3}\right)_{3} \cdot 9 \mathrm{H}_{2} \mathrm{O}(0.0810 \mathrm{~g}, 0.2$ mmol), TEMPO (0.0315 g, $0.2 \mathrm{mmol}), \mathrm{NaCl}(0.0119 \mathrm{~g}, 0.2 \mathrm{mmol}), \mathrm{DCE}(10 \mathrm{~mL})$, and 2-methyltrideca-2,3-dien-1-ol (0.4200 g, $2.0 \mathrm{mmol}) / \mathrm{DCE}(5 \mathrm{~mL})$ for $6 \mathrm{~h}$ afforded 1c $(0.2543 \mathrm{~g}, 61 \%)$ (eluent: petroleum ether $\left(30-60{ }^{\circ} \mathrm{C}\right) /$ ethyl acetate $\left.=100 / 1\right)$ as a 
liquid: ${ }^{1} \mathrm{H}$ NMR $\left(300 \mathrm{MHz}, \mathrm{CDCl}_{3}\right) \delta 9.52(\mathrm{~s}, 1 \mathrm{H}, \mathrm{CHO}), 5.72-5.63(\mathrm{~m}, 1 \mathrm{H},=\mathrm{CH})$, 2.22-2.10 (m, 2H, $\left.\mathrm{CH}_{2}\right), 1.77\left(\mathrm{~d}, J=2.7 \mathrm{~Hz}, 3 \mathrm{H}, \mathrm{CH}_{3}\right), 1.53-1.20\left(\mathrm{~m}, 14 \mathrm{H}, \mathrm{CH}_{2} \times 7\right)$, $0.88\left(\mathrm{t}, J=6.8 \mathrm{~Hz}, 3 \mathrm{H}, \mathrm{CH}_{3}\right) ;{ }^{13} \mathrm{C} \mathrm{NMR}\left(75 \mathrm{MHz}, \mathrm{CDCl}_{3}\right) \delta 218.5,193.0,106.4,95.5$, 31.8, 29.5, 29.3, 29.2, 28.9, 28.8, 27.8, 22.6, 14.0, 10.8; IR (neat) $v\left(\mathrm{~cm}^{-1}\right) 2956,2926$, 2855, 1945, 1687, 1465, 1372, 1221; MS (70 ev, EI) m/z (\%) $208\left(\mathrm{M}^{+}, 7.97\right), 95$ (100); HRMS calcd for $\mathrm{C}_{14} \mathrm{H}_{24} \mathrm{O}\left[\mathrm{M}^{+}\right]$: 208.1827, found: 208.1829.

\section{2-Propylundeca-2,3-dienal (1d) (djx-3-009)}

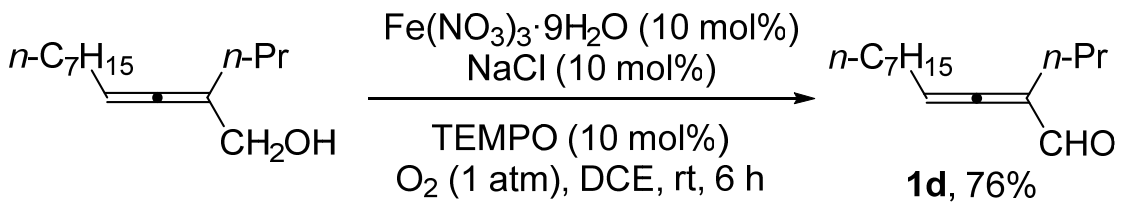

Following Typical Procedure I, the reaction of $\mathrm{Fe}\left(\mathrm{NO}_{3}\right)_{3} \cdot 9 \mathrm{H}_{2} \mathrm{O}(0.0809 \mathrm{~g}, 0.2$ mmol), TEMPO (0.0313 g, $0.2 \mathrm{mmol}), \mathrm{NaCl}(0.0118 \mathrm{~g}, 0.2 \mathrm{mmol}), \mathrm{DCE}(10 \mathrm{~mL})$, and 2-propylundeca-2,3-dien-1-ol (0.4201 g, $2.0 \mathrm{mmol}) / \mathrm{DCE}(3 \mathrm{~mL})$ for $6 \mathrm{~h}$ afforded 1d $(0.3175 \mathrm{~g}, 76 \%)$ (eluent: petroleum ether $\left(30-60{ }^{\circ} \mathrm{C}\right) / \mathrm{ethyl}$ acetate $\left.=100 / 1\right)$ as a liquid: ${ }^{1} \mathrm{H}$ NMR $\left(300 \mathrm{MHz}, \mathrm{CDCl}_{3}\right) \delta 9.52(\mathrm{~s}, 1 \mathrm{H}, \mathrm{CHO}), 5.76-5.67(\mathrm{~m}, 1 \mathrm{H},=\mathrm{CH})$, 2.25-2.05 (m, 4H, $\left.\mathrm{CH}_{2} \times 2\right), 1.55-1.20\left(\mathrm{~m}, 12 \mathrm{H}, \mathrm{CH}_{2} \times 6\right), 0.98-0.84\left(\mathrm{~m}, 6 \mathrm{H}, \mathrm{CH}_{3} \times 2\right)$; ${ }^{13} \mathrm{C}$ NMR $\left(75 \mathrm{MHz}, \mathrm{CDCl}_{3}\right) \delta$ 218.4, 192.8, 111.5, 96.4, 31.7, 28.93, 28.88, 27.9, 26.6, 22.5, 20.9, 14.0, 13.6; IR (neat) $v\left(\mathrm{~cm}^{-1}\right)$ 2959, 2928, 2856, 2801, 2756, 2694, 1943, 1686, 1463, 1379, 1209, 1175, 1093; MS (70 ev, EI) $m / z(\%) 208\left(\mathrm{M}^{+}, 1.34\right), 95$ (100); HRMS calcd for $\mathrm{C}_{14} \mathrm{H}_{24} \mathrm{O}\left[\mathrm{M}^{+}\right]$: 208.1827, found: 208.1828 .

5. 2-Propyltrideca-2,3-dienal (1e) (djx-3-014)

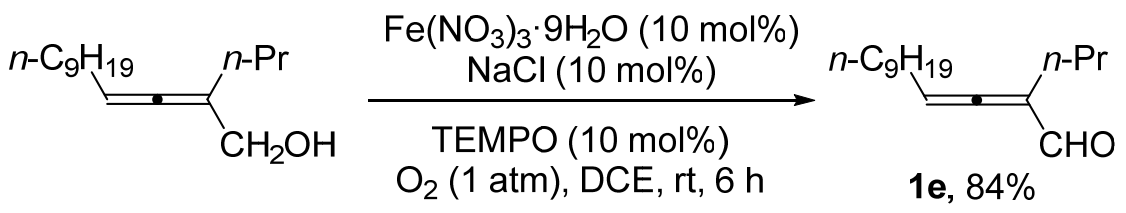

Following Typical Procedure I, the reaction of $\mathrm{Fe}\left(\mathrm{NO}_{3}\right)_{3} \cdot 9 \mathrm{H}_{2} \mathrm{O}(0.0810 \mathrm{~g}, 0.2$ mmol), TEMPO (0.0313 g, $0.2 \mathrm{mmol}), \mathrm{NaCl}(0.0118 \mathrm{~g}, 0.2 \mathrm{mmol}), \mathrm{DCE}(10 \mathrm{~mL})$, and 2-propyltrideca-2,3-dien-1-ol (0.4761 g, $2.0 \mathrm{mmol}) / \mathrm{DCE}(3 \mathrm{~mL})$ for $6 \mathrm{~h}$ afforded 1e $(0.3978 \mathrm{~g}, 84 \%)$ (eluent: petroleum ether $\left(30-60{ }^{\circ} \mathrm{C}\right) /$ ethyl acetate $\left.=100 / 1\right)$ as a 
liquid: ${ }^{1} \mathrm{H}$ NMR $\left(300 \mathrm{MHz}, \mathrm{CDCl}_{3}\right) \delta 9.52(\mathrm{~s}, 1 \mathrm{H}, \mathrm{CHO})$, 5.76-5.67 (m, 1H, $\left.=\mathrm{CH}\right)$, 2.25-2.05 (m, 4H, $\left.\mathrm{CH}_{2} \times 2\right), 1.54-1.20\left(\mathrm{~m}, 16 \mathrm{H}, \mathrm{CH}_{2} \times 8\right), 0.98-0.82\left(\mathrm{~m}, 6 \mathrm{H}, \mathrm{CH}_{3} \times 2\right)$; ${ }^{13} \mathrm{C}$ NMR $\left(75 \mathrm{MHz}, \mathrm{CDCl}_{3}\right) \delta 218.4,192.8,111.5,96.4,31.8,29.5,29.3,29.2,28.9$, 27.9, 26.6, 22.6, 20.9, 14.0, 13.7; IR (neat) $v\left(\mathrm{~cm}^{-1}\right)$ 2959, 2926, 2855, 2806, 2755, 2699, 1942, 1687, 1463, 1378, 1207, 1174, 1091; MS (70 ev, EI) m/z (\%) $236\left(\mathrm{M}^{+}\right.$, 3.50), 123 (100); HRMS calcd for $\mathrm{C}_{16} \mathrm{H}_{28} \mathrm{O}\left[\mathrm{M}^{+}\right]$: 236.2140, found: 236.2142.

\section{2-Propyltetradeca-2,3-dienal (1f) (djx-3-045)}

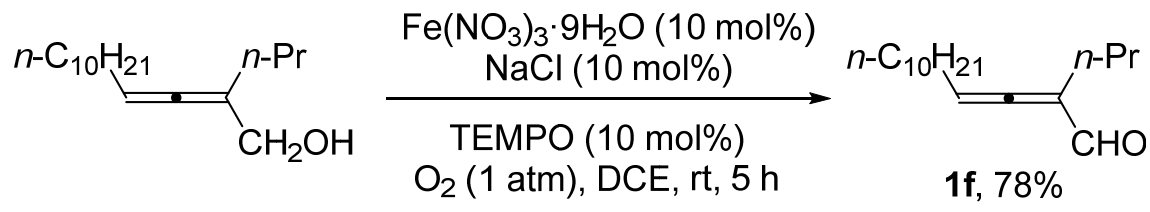

Following Typical Procedure I, the reaction of $\mathrm{Fe}\left(\mathrm{NO}_{3}\right)_{3} \cdot 9 \mathrm{H}_{2} \mathrm{O}(0.1211 \mathrm{~g}, 0.3$ mmol), TEMPO (0.0468 g, $0.3 \mathrm{mmol}), \mathrm{NaCl}(0.0177 \mathrm{~g}, 0.3 \mathrm{mmol}), \mathrm{DCE}(15 \mathrm{~mL})$, and 2-propyltetradeca-2,3-dien-1-ol $(0.7557 \mathrm{~g}, 3.0 \mathrm{mmol}) / \mathrm{DCE}(5 \mathrm{~mL})$ for $5 \mathrm{~h}$ afforded 1f $(0.5851 \mathrm{~g}, 78 \%)$ (eluent: petroleum ether $\left(30-60{ }^{\circ} \mathrm{C}\right) /$ diethyl ether $\left.=100 / 1\right)$ as a liquid: ${ }^{1} \mathrm{H}$ NMR $\left(300 \mathrm{MHz}, \mathrm{CDCl}_{3}\right) \delta 9.52(\mathrm{~s}, 1 \mathrm{H}, \mathrm{CHO}), 5.76-5.67(\mathrm{~m}, 1 \mathrm{H}$, $=\mathrm{CH}), 2.24-2.06\left(\mathrm{~m}, 4 \mathrm{H}, \mathrm{CH}_{2} \times 2\right), 1.53-1.20\left(\mathrm{~m}, 18 \mathrm{H}, \mathrm{CH}_{2} \times 9\right), 0.93(\mathrm{t}, J=7.4 \mathrm{~Hz}$, $\left.3 \mathrm{H}, \mathrm{CH}_{3}\right), 0.88\left(\mathrm{t}, J=6.5 \mathrm{~Hz}, 3 \mathrm{H}, \mathrm{CH}_{3}\right) ;{ }^{13} \mathrm{C} \mathrm{NMR}\left(75 \mathrm{MHz}, \mathrm{CDCl}_{3}\right) \delta 218.5,192.8$, $111.5,96.4,31.8,29.5,29.29,29.27,29.0,28.0,26.6,22.6,21.0,14.0,13.7$; IR (neat) $v\left(\mathrm{~cm}^{-1}\right) 2958,2926,2855,2806,2755,2699,1942,1687,1464,1378,1206,1174$, 1084; MS (70 ev, EI) $m / z(\%) 250\left(\mathrm{M}^{+}, 23.33\right), 137$ (100); HRMS calcd for $\mathrm{C}_{17} \mathrm{H}_{30} \mathrm{O}$ $\left[\mathrm{M}^{+}\right]: 250.2297$, found: 250.2299 .

\section{2-Propyl-4-cyclohexylbuta-2,3-dienal (1g) (djx-2-197)}
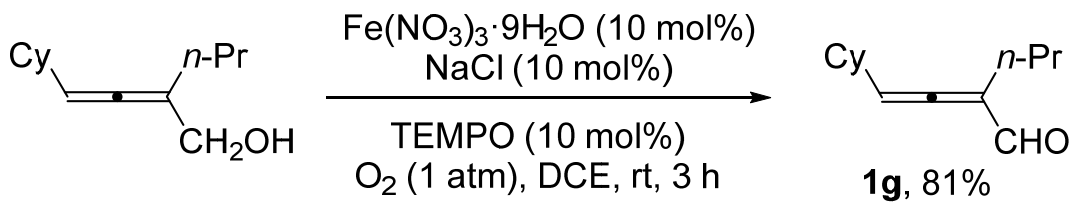

1g, $81 \%$

Following Typical Procedure I, the reaction of $\mathrm{Fe}\left(\mathrm{NO}_{3}\right)_{3} \cdot 9 \mathrm{H}_{2} \mathrm{O}(0.1214 \mathrm{~g}, 0.3$ mmol), TEMPO (0.0470 g, $0.3 \mathrm{mmol}), \mathrm{NaCl}(0.0177 \mathrm{~g}, 0.3 \mathrm{mmol}), \mathrm{DCE}(15 \mathrm{~mL})$, and 2-propyl-4-cyclohexylbuta-2,3-dien-1-ol (0.5822 g, $3.0 \mathrm{mmol}) / \mathrm{DCE}(5 \mathrm{~mL})$ for 3 
$\mathrm{h}$ afforded $1 \mathrm{~g}(0.4660 \mathrm{~g}, 81 \%)$ (eluent: petroleum ether $\left(30-60{ }^{\circ} \mathrm{C}\right) /$ ethyl acetate $=$ 100/1) as a liquid: ${ }^{1} \mathrm{H}$ NMR $\left(300 \mathrm{MHz}, \mathrm{CDCl}_{3}\right) \delta 9.49$ (s, 1H, CHO), 5.78-5.70 (m, $1 \mathrm{H},=\mathrm{CH}), 2.26-2.05\left(\mathrm{~m}, 3 \mathrm{H}, \mathrm{CH}\right.$ and $\left.\mathrm{CH}_{2}\right), 1.90-1.60(\mathrm{~m}, 5 \mathrm{H}, 5 \mathrm{H}$ from $\mathrm{Cy})$, 1.54-1.06 (m, 7H, 5H from Cy and $\left.\mathrm{CH}_{2}\right), 0.93\left(\mathrm{t}, J=7.4 \mathrm{~Hz}, 3 \mathrm{H}, \mathrm{CH}_{3}\right) ;{ }^{13} \mathrm{C} \mathrm{NMR}(75$ $\left.\mathrm{MHz}, \mathrm{CDCl}_{3}\right) \delta 217.6,192.5,112.5,102.2,37.0,32.9,26.5,25.7,20.9,13.6$; IR (neat) $v\left(\mathrm{~cm}^{-1}\right)$ 2962, 2927, 2853, 2798, 1941, 1684, 1449, 1231, 1208, 1180, 1090; MS (70 ev, EI) $m / z(\%) 192\left(\mathrm{M}^{+}, 11.54\right), 149$ (100); HRMS calcd for $\mathrm{C}_{13} \mathrm{H}_{20} \mathrm{O}\left[\mathrm{M}^{+}\right]: 192.1514$, found: 192.1515 .

8. 5-Phenyl-2-propylpenta-2,3-dienal (1h) (djx-3-061)

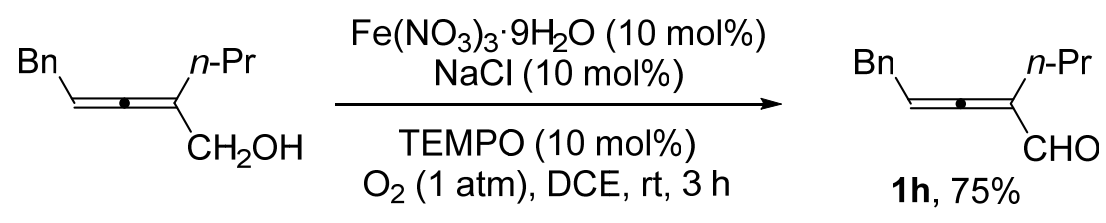

Following Typical Procedure I, the reaction of $\mathrm{Fe}\left(\mathrm{NO}_{3}\right)_{3} \cdot 9 \mathrm{H}_{2} \mathrm{O}(0.1215 \mathrm{~g}, 0.3$ mmol), TEMPO (0.0471 g, $0.3 \mathrm{mmol}), \mathrm{NaCl}$ (0.0176 g, $0.3 \mathrm{mmol}), \mathrm{DCE}(15 \mathrm{~mL})$, and 5-phenyl-2-propylpenta-2,3-dien-1-ol (0.6058 g, $3.0 \mathrm{mmol}) / \mathrm{DCE}(5 \mathrm{~mL})$ for $3 \mathrm{~h}$ afforded $\mathbf{1 h}(0.4511 \mathrm{~g}, 75 \%)$ (eluent: petroleum ether $\left(30-60{ }^{\circ} \mathrm{C}\right) /$ diethyl ether $\left.=100 / 1\right)$ as a liquid: ${ }^{1} \mathrm{H} \mathrm{NMR}\left(300 \mathrm{MHz}, \mathrm{CDCl}_{3}\right) \delta 9.51(\mathrm{~s}, 1 \mathrm{H}, \mathrm{CHO}), 7.35-7.17$ (m, 5H, ArH), $5.88\left(\mathrm{tt}, J_{1}=7.2 \mathrm{~Hz}, J_{2}=4.8 \mathrm{~Hz}, 1 \mathrm{H},=\mathrm{CH}\right), 3.48\left(\mathrm{~d}, J=7.2 \mathrm{~Hz}, 2 \mathrm{H}, \mathrm{CH}_{2}\right), 2.21-2.02$ $\left(\mathrm{m}, 2 \mathrm{H}, \mathrm{CH}_{2}\right), 1.41$ (sextet, $\left.J=7.4 \mathrm{~Hz}, 2 \mathrm{H}, \mathrm{CH}_{2}\right), 0.89$ (t, $\left.J=7.4 \mathrm{~Hz}, 3 \mathrm{H}, \mathrm{CH}_{3}\right) ;{ }^{13} \mathrm{C}$ $\mathrm{NMR}\left(75 \mathrm{MHz}, \mathrm{CDCl}_{3}\right) \delta 218.6,192.3,138.5,128.5,128.3,126.6,111.7,96.2,34.5$, 26.4, 20.8, 13.6; IR (neat) $v\left(\mathrm{~cm}^{-1}\right)$ 3085, 3063, 3028, 2961, 2931, 2872, 2837, 2807, 2753, 2701, 1943, 1682, 1603, 1495, 1454, 1435, 1379, 1212, 1193, 1074, 1030; MS (70 ev, EI) $m / z(\%) 200\left(\mathrm{M}^{+}, 21.90\right), 91$ (100); HRMS calcd for $\mathrm{C}_{14} \mathrm{H}_{16} \mathrm{O}\left[\mathrm{M}^{+}\right]$: 200.1201, found: 200.1201 .

9. 7-Chloro-2-propylhepta-2,3-dienal (1i) (djx-3-049)

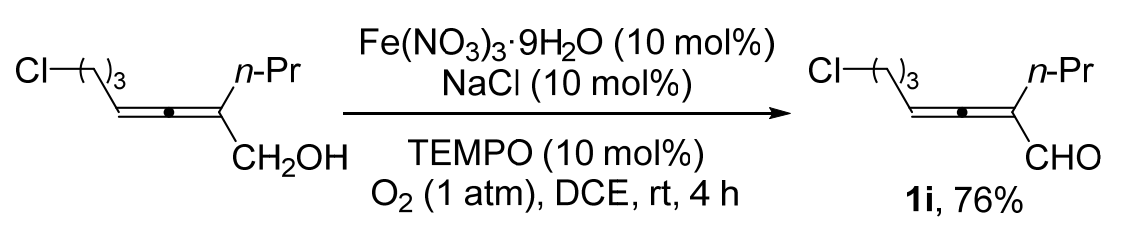


Following Typical Procedure I, the reaction of $\mathrm{Fe}\left(\mathrm{NO}_{3}\right)_{3} \cdot 9 \mathrm{H}_{2} \mathrm{O}(0.1415 \mathrm{~g}, 0.35$ mmol), TEMPO (0.0546 g, $0.35 \mathrm{mmol}), \mathrm{NaCl}(0.0207 \mathrm{~g}, 0.35 \mathrm{mmol}), \mathrm{DCE}(20 \mathrm{~mL})$, and 7-chloro-2-propylhepta-2,3-dien-1-ol (0.6612 g, $3.5 \mathrm{mmol}) / \mathrm{DCE}(5 \mathrm{~mL})$ for $4 \mathrm{~h}$ afforded 1i $(0.5003 \mathrm{~g}, 76 \%)$ (eluent: petroleum ether $\left(30-60{ }^{\circ} \mathrm{C}\right) /$ diethyl ether $\left.=50 / 1\right)$ as a liquid: ${ }^{1} \mathrm{H}$ NMR $\left(300 \mathrm{MHz}, \mathrm{CDCl}_{3}\right) \delta 9.53(\mathrm{~s}, 1 \mathrm{H}, \mathrm{CHO}), 5.79-5.70(\mathrm{~m}, 1 \mathrm{H}$, $=\mathrm{CH}), 3.62\left(\mathrm{t}, J=6.5 \mathrm{~Hz}, 2 \mathrm{H}, \mathrm{CH}_{2}\right), 2.42-2.31\left(\mathrm{~m}, 2 \mathrm{H}, \mathrm{CH}_{2}\right), 2.20-2.12\left(\mathrm{~m}, 2 \mathrm{H}, \mathrm{CH}_{2}\right)$, 2.01-1.89 (m, 2H, $\mathrm{CH}_{2}$ ), 1.46 (sextet, $J=7.4 \mathrm{~Hz}, 2 \mathrm{H}, \mathrm{CH}_{2}$ ), 0.93 (t, $J=7.4 \mathrm{~Hz}, 3 \mathrm{H}$, $\left.\mathrm{CH}_{3}\right) ;{ }^{13} \mathrm{C} \mathrm{NMR}\left(75 \mathrm{MHz}, \mathrm{CDCl}_{3}\right) \delta 218.2,192.3,111.9,95.0,43.8,31.4,26.5,25.1$, 20.9, 13.6; IR (neat) $v\left(\mathrm{~cm}^{-1}\right) 2961,2932,2872,2810,2758,2704,1943,1682,1445$, 1373, 1309, 1288, 1250, 1212, 1174, 1085; MS (70 ev, EI) $m / z(\%) 188\left(\mathrm{M}^{+}\left({ }^{37} \mathrm{Cl}\right)\right.$, 6.08), $186\left(\mathrm{M}^{+}\left({ }^{35} \mathrm{Cl}\right), 19.28\right), 123(100)$; HRMS calcd for $\mathrm{C}_{10} \mathrm{H}_{15} \mathrm{O}^{35} \mathrm{Cl}\left[\mathrm{M}^{+}\right]$: 186.0811 , found: 186.0814 .

10. 2-Propyltrideca-2,3,12-trienal (1j) (djx-3-030)

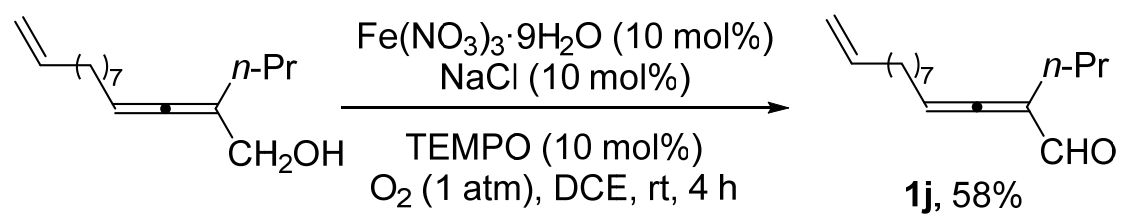

Following Typical Procedure I, the reaction of $\mathrm{Fe}\left(\mathrm{NO}_{3}\right)_{3} \cdot 9 \mathrm{H}_{2} \mathrm{O}(0.1213 \mathrm{~g}, 0.3$ mmol), TEMPO (0.0469 g, $0.3 \mathrm{mmol}), \mathrm{NaCl}(0.0177 \mathrm{~g}, 0.3 \mathrm{mmol}), \mathrm{DCE}(15 \mathrm{~mL})$, and 2-propyltrideca-2,3,12-trien-1-ol $(0.7070 \mathrm{~g}, 3.0 \mathrm{mmol}) / \mathrm{DCE}(5 \mathrm{~mL})$ for $4 \mathrm{~h}$ afforded 1j (0.4212 g, 58\%, purity: 96\%) (eluent: petroleum ether $\left(30-60{ }^{\circ} \mathrm{C}\right) /$ diethyl ether $=200 / 1)$ as a liquid: ${ }^{1} \mathrm{H}$ NMR $\left(300 \mathrm{MHz}, \mathrm{CDCl}_{3}\right) \delta 9.52(\mathrm{~s}, 1 \mathrm{H}, \mathrm{CHO})$, 5.89-5.66 (m, $2 \mathrm{H},=\mathrm{CH} \times 2), 5.05-4.88\left(\mathrm{~m}, 2 \mathrm{H},=\mathrm{CH}_{2}\right), 2.22-1.96\left(\mathrm{~m}, 6 \mathrm{H}, \mathrm{CH}_{2} \times 3\right)$, 1.55-1.20 (m, $\left.12 \mathrm{H}, \mathrm{CH}_{2} \times 6\right), 0.93\left(\mathrm{t}, J=7.5 \mathrm{~Hz}, 3 \mathrm{H}, \mathrm{CH}_{3}\right) ;{ }^{13} \mathrm{C} \mathrm{NMR}(75 \mathrm{MHz}$, $\left.\mathrm{CDCl}_{3}\right) \delta 218.5,192.8,139.0,114.2,111.5,96.4,33.7,29.1,28.95,28.92,28.87,28.8$, 27.9, 26.6, 20.9, 13.7; IR (neat) $v\left(\mathrm{~cm}^{-1}\right)$ 3077, 2960, 2928, 2855, 2804, 2755, 2701, 1943, 1720, 1686, 1640, 1463, 1206, 1175; MS (70 ev, EI) $m / z(\%) 234\left(\mathrm{M}^{+}, 1.10\right), 41$ (100); HRMS calcd for $\mathrm{C}_{16} \mathrm{H}_{26} \mathrm{O}\left[\mathrm{M}^{+}\right]$: 234.1984, found: 234.1991 .

11. 2-Propyl-4-(p-tolyl)buta-2,3-dienal (1k) (djx-3-181) 


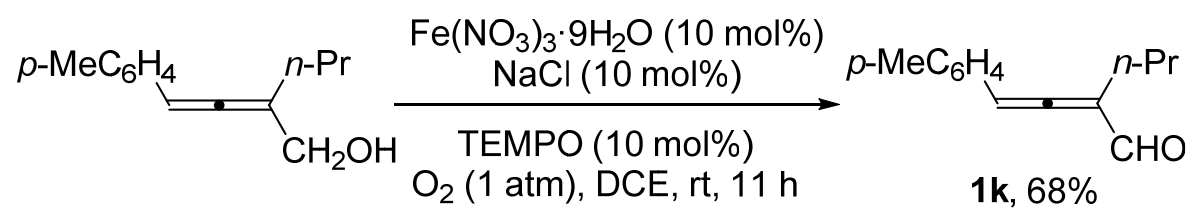

Following Typical Procedure I, the reaction of $\mathrm{Fe}\left(\mathrm{NO}_{3}\right)_{3} \cdot 9 \mathrm{H}_{2} \mathrm{O}(0.1613 \mathrm{~g}, 0.4$ mmol), TEMPO (0.0622 g, $0.4 \mathrm{mmol}), \mathrm{NaCl}(0.0231 \mathrm{~g}, 0.4 \mathrm{mmol}), \mathrm{DCE}(20 \mathrm{~mL})$, and 2-propyl-4-(p-tolyl)buta-2,3-dien-1-ol (0.8032 g, $4.0 \mathrm{mmol}) / \mathrm{DCE}(5 \mathrm{~mL})$ for $11 \mathrm{~h}$ afforded 1k (0.5438 g, 68\%) (eluent: petroleum ether $\left(30-60{ }^{\circ} \mathrm{C}\right) /$ diethyl ether $\left.=100 / 1\right)$ as a liquid: ${ }^{1} \mathrm{H} \mathrm{NMR}\left(300 \mathrm{MHz}, \mathrm{CDCl}_{3}\right) \delta 9.61(\mathrm{~s}, 1 \mathrm{H}, \mathrm{CHO}), 7.24-7.08(\mathrm{~m}, 4 \mathrm{H}, \mathrm{ArH})$, $6.71(\mathrm{t}, J=2.9 \mathrm{~Hz}, 1 \mathrm{H},=\mathrm{CH}), 2.41-2.19\left(\mathrm{~m}, 5 \mathrm{H}, \mathrm{CH}_{3}\right.$ and $\left.\mathrm{CH}_{2}\right), 1.60-1.45(\mathrm{~m}, 2 \mathrm{H}$, $\left.\mathrm{CH}_{2}\right), 0.95\left(\mathrm{t}, J=7.4 \mathrm{~Hz}, 3 \mathrm{H}, \mathrm{CH}_{3}\right) ;{ }^{13} \mathrm{C} \mathrm{NMR}\left(75 \mathrm{MHz}, \mathrm{CDCl}_{3}\right) \delta 220.6,191.7$, 138.3, 129.7, 128.5, 127.2, 114.9, 99.6, 27.0, 21.2, 21.1, 13.8; IR (neat) $v\left(\mathrm{~cm}^{-1}\right) 3021$, 2961, 2930, 2872, 1929, 1715, 1683, 1513, 1462, 1456, 1202, 1192, 1162, 1089, 1052; MS (70 ev, EI) $m / z(\%) 200\left(\mathrm{M}^{+}, 32.32\right), 128$ (100); HRMS calcd for $\mathrm{C}_{14} \mathrm{H}_{16} \mathrm{O}\left[\mathrm{M}^{+}\right]$: 200.1201, found: 200.1208 .

12. 2-Butyl-4-phenylbuta-2,3-dienal (1l) (djx-2-192)
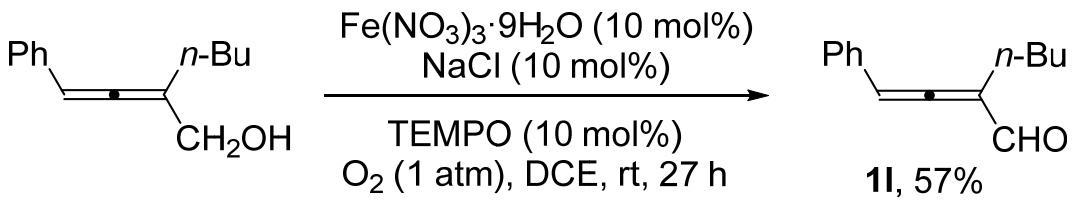

Following Typical Procedure I, the reaction of $\mathrm{Fe}\left(\mathrm{NO}_{3}\right)_{3} \cdot 9 \mathrm{H}_{2} \mathrm{O}(0.1213 \mathrm{~g}, 0.3$ mmol), TEMPO (0.0469 g, $0.3 \mathrm{mmol}), \mathrm{NaCl}(0.0177 \mathrm{~g}, 0.3 \mathrm{mmol}), \mathrm{DCE}(15 \mathrm{~mL})$, and 2-butyl-4-phenylbuta-2,3-dien-1-ol (0.6062 g, $3.0 \mathrm{mmol}) / \mathrm{DCE}(5 \mathrm{~mL})$ for $27 \mathrm{~h}$ afforded 11 (0.3401 g, 57\%) (eluent: petroleum ether $\left(30-60{ }^{\circ} \mathrm{C}\right) /$ diethyl ether $\left.=100 / 1\right)$ as a liquid: ${ }^{1} \mathrm{H}$ NMR (300 MHz, $\left.\mathrm{CDCl}_{3}\right) \delta 9.62(\mathrm{~s}, 1 \mathrm{H}, \mathrm{CHO}), 7.41-7.23(\mathrm{~m}, 5 \mathrm{H}, \mathrm{ArH})$, $6.74(\mathrm{t}, J=2.7 \mathrm{~Hz}, 1 \mathrm{H},=\mathrm{CH}), 2.44-2.22\left(\mathrm{~m}, 2 \mathrm{H}, \mathrm{CH}_{2}\right), 1.56-1.30\left(\mathrm{~m}, 4 \mathrm{H}, \mathrm{CH}_{2} \times 2\right)$, $0.90\left(\mathrm{t}, J=7.4 \mathrm{~Hz}, 3 \mathrm{H}, \mathrm{CH}_{3}\right) ;{ }^{13} \mathrm{C} \mathrm{NMR}\left(75 \mathrm{MHz}, \mathrm{CDCl}_{3}\right) \delta 220.5,191.6,131.5$, 128.9, 128.2, 127.2, 115.1, 99.8, 29.8, 24.6, 22.4, 13.8; IR (neat) $v\left(\mathrm{~cm}^{-1}\right) 3085,3064$, 3032, 2957, 2929, 2872, 2860, 2752, 2705, 1931, 1686, 1599, 1497, 1459, 1201, 1158, 1072, 1028; MS (70 ev, EI) m/z (\%) $200\left(\mathrm{M}^{+}, 6.06\right), 129$ (100); HRMS calcd for $\mathrm{C}_{14} \mathrm{H}_{16} \mathrm{O}\left[\mathrm{M}^{+}\right]: 200.1201$, found: 200.1200 . 
13. 2-Butyl-4-(p-tolyl)buta-2,3-dienal (1m) (djx-3-142)

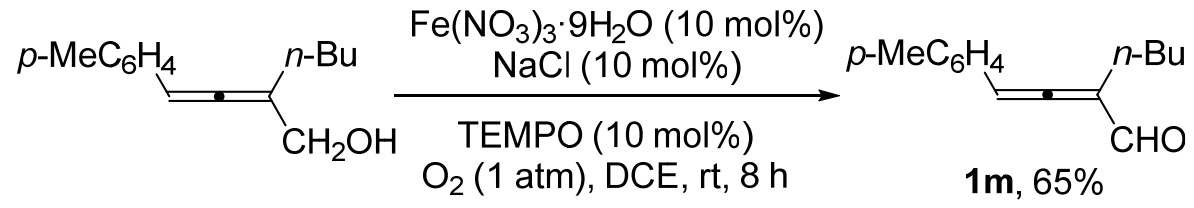

Following Typical Procedure I, the reaction of $\mathrm{Fe}\left(\mathrm{NO}_{3}\right)_{3} \cdot 9 \mathrm{H}_{2} \mathrm{O}(0.1857 \mathrm{~g}, 0.46$ mmol), TEMPO (0.0719 g, $0.46 \mathrm{mmol}), \mathrm{NaCl}(0.0270 \mathrm{~g}, 0.46 \mathrm{mmol}), \mathrm{DCE}(25 \mathrm{~mL})$, and 2-butyl-4-(p-tolyl)buta-2,3-dien-1-ol (0.9926 g, $4.6 \mathrm{mmol}) / \mathrm{DCE}(5 \mathrm{~mL})$ for $8 \mathrm{~h}$ afforded 1m (0.6385 g, 65\%) (eluent: petroleum ether $\left(30-60{ }^{\circ} \mathrm{C}\right) /$ diethyl ether $=$ 100/1) as a liquid: ${ }^{1} \mathrm{H}$ NMR $\left(300 \mathrm{MHz}, \mathrm{CDCl}_{3}\right) \delta 9.60(\mathrm{~s}, 1 \mathrm{H}, \mathrm{CHO}), 7.23-7.13(\mathrm{~m}$, $4 \mathrm{H}, \mathrm{ArH}), 6.71(\mathrm{t}, J=2.7 \mathrm{~Hz}, 1 \mathrm{H},=\mathrm{CH}), 2.43-2.18\left(\mathrm{~m}, 5 \mathrm{H}, \mathrm{CH}_{3}\right.$ and $\left.\mathrm{CH}_{2}\right), 1.55-1.28$ $\left(\mathrm{m}, 4 \mathrm{H}, \mathrm{CH}_{2} \times 2\right), 0.90\left(\mathrm{t}, J=7.2 \mathrm{~Hz}, 3 \mathrm{H}, \mathrm{CH}_{3}\right) ;{ }^{13} \mathrm{C} \mathrm{NMR}\left(75 \mathrm{MHz}, \mathrm{CDCl}_{3}\right) \delta 220.5$, 191.7, 138.2, 129.7, 128.5, 127.1, 115.1, 99.7, 29.8, 24.7, 22.4, 21.2, 13.8; IR (neat) $v$ $\left(\mathrm{cm}^{-1}\right)$ 2957, 2928, 2872, 2860, 1929, 1686, 1513, 1465, 1456, 1199, 1189; MS (70 ev, EI) $m / z(\%) 214\left(\mathrm{M}^{+}, 10.30\right), 143(100)$; HRMS calcd for $\mathrm{C}_{15} \mathrm{H}_{18} \mathrm{O}\left[\mathrm{M}^{+}\right]$: 214.1358, found: 214.1357.

14. 2-Allyltrideca-2,3-dienal (1n) (djx-3-066)

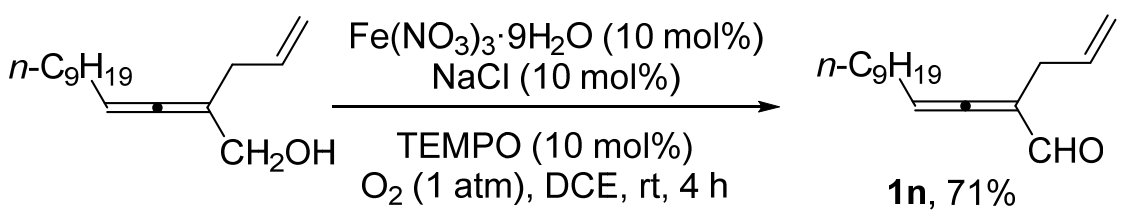

Following Typical Procedure I, the reaction of $\mathrm{Fe}\left(\mathrm{NO}_{3}\right)_{3} \cdot 9 \mathrm{H}_{2} \mathrm{O}(0.1215 \mathrm{~g}, 0.3$ mmol), TEMPO (0.0473 g, $0.3 \mathrm{mmol}), \mathrm{NaCl}(0.0177 \mathrm{~g}, 0.3 \mathrm{mmol}), \mathrm{DCE}(15 \mathrm{~mL})$, and 2-allyltrideca-2,3-dien-1-ol (0.7085 g, $3.0 \mathrm{mmol}) / \mathrm{DCE}(5 \mathrm{~mL})$ for $4 \mathrm{~h}$ afforded $\mathbf{1 n}$ $(0.4984 \mathrm{~g}, 71 \%)$ (eluent: petroleum ether $\left(30-60{ }^{\circ} \mathrm{C}\right) /$ diethyl ether $\left.=200 / 1\right)$ as a liquid: ${ }^{1} \mathrm{H}$ NMR $\left(300 \mathrm{MHz}, \mathrm{CDCl}_{3}\right) \delta 9.53(\mathrm{~s}, 1 \mathrm{H}, \mathrm{CHO}), 5.90-5.68(\mathrm{~m}, 2 \mathrm{H},=\mathrm{CH} \times 2)$, 5.19-4.96 (m, 2H, = $\left.\mathrm{CH}_{2}\right), 2.94\left(\mathrm{~d}, J=5.7 \mathrm{~Hz}, 2 \mathrm{H}, \mathrm{CH}_{2}\right), 2.28-2.08\left(\mathrm{~m}, 2 \mathrm{H}, \mathrm{CH}_{2}\right)$, $1.58-1.18\left(\mathrm{~m}, 14 \mathrm{H}, \mathrm{CH}_{2} \times 7\right), 0.88\left(\mathrm{t}, J=6.3 \mathrm{~Hz}, 3 \mathrm{H}, \mathrm{CH}_{3}\right) ;{ }^{13} \mathrm{C} \mathrm{NMR}(75 \mathrm{MHz}$, $\left.\mathrm{CDCl}_{3}\right) \delta 218.4,192.0,134.6,116.3,109.9,97.1,31.8,29.5,29.3,29.2,28.9,28.8$, 27.8, 22.6, 14.0; IR (neat) $v\left(\mathrm{~cm}^{-1}\right) 3081,2926,2855,2810,2750,2694,1943,1686$, 1644, 1465, 1435, 1202, 1163; MS (70 ev, EI) m/z (\%) $234\left(\mathrm{M}^{+}, 17.33\right), 121$ (100); 
HRMS calcd for $\mathrm{C}_{16} \mathrm{H}_{26} \mathrm{O}\left[\mathrm{M}^{+}\right]$: 234.1984, found: 234.1982.

15. 2-Benzylpenta-2,3-dienal (10) (djx-3-022)

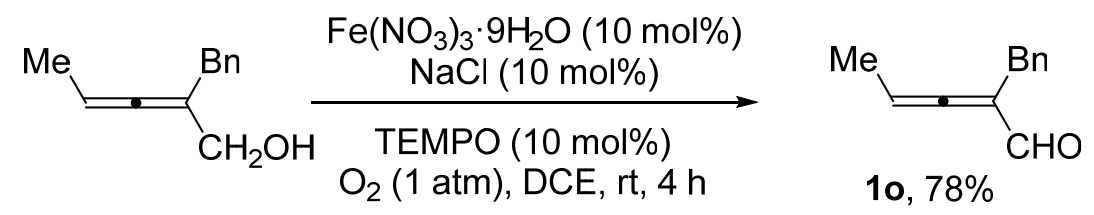

Following Typical Procedure I, the reaction of $\mathrm{Fe}\left(\mathrm{NO}_{3}\right)_{3} \cdot 9 \mathrm{H}_{2} \mathrm{O}(0.0688 \mathrm{~g}, 0.17$ mmol), TEMPO (0.0266 g, $0.17 \mathrm{mmol}), \mathrm{NaCl}(0.0100 \mathrm{~g}, 0.17 \mathrm{mmol}), \mathrm{DCE}(10 \mathrm{~mL})$, and 2-benzylpenta-2,3-dien-1-ol (0.2954 g, $1.7 \mathrm{mmol}) / \mathrm{DCE}(3 \mathrm{~mL})$ for $4 \mathrm{~h}$ afforded $10(0.2174 \mathrm{~g}, 78 \%)$ (eluent: petroleum ether $\left(30-60{ }^{\circ} \mathrm{C}\right) /$ diethyl ether $\left.=100 / 1\right)$ as a liquid: ${ }^{1} \mathrm{H}$ NMR (300 MHz, $\left.\mathrm{CDCl}_{3}\right) \delta 9.55$ (s, 1H, CHO), 7.34-7.14 (m, 5H, ArH), 5.72-5.59 (m, $1 \mathrm{H},=\mathrm{CH}), 3.59-3.42\left(\mathrm{~m}, 2 \mathrm{H}, \mathrm{CH}_{2}\right), 1.75\left(\mathrm{~d}, J=7.5 \mathrm{~Hz}, 3 \mathrm{H}, \mathrm{CH}_{3}\right) ;{ }^{13} \mathrm{C}$ NMR (75 MHz, $\left.\mathrm{CDCl}_{3}\right) \delta 219.3,191.9,138.9,128.8,128.2,126.2,110.8,91.8,31.2$, 13.0; IR (neat) $v\left(\mathrm{~cm}^{-1}\right)$ 3085, 3062, 3028, 2979, 2955, 2926, 2829, 2800, 2743, 2692, 1946, 1682, 1602, 1495, 1453, 1371, 1205, 1145, 1073, 1030; MS (70 ev, EI) m/z (\%) $172\left(\mathrm{M}^{+}, 23.35\right), 129$ (100); HRMS calcd for $\mathrm{C}_{12} \mathrm{H}_{12} \mathrm{O}\left[\mathrm{M}^{+}\right]$: 172.0888, found: 172.0886 .

16. 2-Benzylhepta-2,3-dienal (1p) (djx-3-033)

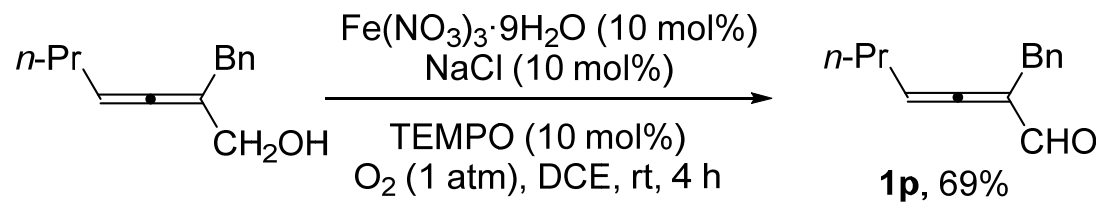

Following Typical Procedure I, the reaction of $\mathrm{Fe}\left(\mathrm{NO}_{3}\right)_{3} \cdot 9 \mathrm{H}_{2} \mathrm{O}(0.1215 \mathrm{~g}, 0.3$ mmol), TEMPO (0.0469 g, $0.3 \mathrm{mmol}), \mathrm{NaCl}(0.0177 \mathrm{~g}, 0.3 \mathrm{mmol}), \mathrm{DCE}(15 \mathrm{~mL})$, and 2-benzylhepta-2,3-dien-1-ol (0.6050 g, $3.0 \mathrm{mmol}) / \mathrm{DCE}(5 \mathrm{~mL})$ for $4 \mathrm{~h}$ afforded $1 p(0.4153 \mathrm{~g}, 69 \%)$ (eluent: petroleum ether $\left(30-60{ }^{\circ} \mathrm{C}\right) /$ diethyl ether $\left.=50 / 1\right)$ as a liquid: ${ }^{1} \mathrm{H}$ NMR (300 MHz, $\left.\mathrm{CDCl}_{3}\right) \delta 9.55$ (s, 1H, CHO), 7.30-7.12 (m, 5H, ArH), $5.65\left(\mathrm{tt}, J_{1}=7.2 \mathrm{~Hz}, J_{2}=4.8 \mathrm{~Hz}, 1 \mathrm{H},=\mathrm{CH}\right), 3.58-3.42\left(\mathrm{~m}, 2 \mathrm{H}, \mathrm{CH}_{2}\right), 2.15-1.95(\mathrm{~m}$, $\left.2 \mathrm{H}, \mathrm{CH}_{2}\right), 1.37$ (sext, $\left.J=7.4 \mathrm{~Hz}, 2 \mathrm{H}, \mathrm{CH}_{2}\right), 0.87\left(\mathrm{t}, J=7.4 \mathrm{~Hz}, 3 \mathrm{H}, \mathrm{CH}_{3}\right) ;{ }^{13} \mathrm{C} \mathrm{NMR}^{\mathrm{N}}$ $\left(75 \mathrm{MHz}, \mathrm{CDCl}_{3}\right) \delta 218.7,191.9,138.9,128.8,128.2,126.1,111.4,96.9,31.2,29.7$, 
22.0, 13.4; IR (neat) $v\left(\mathrm{~cm}^{-1}\right) 3084,3063,3028,2960,2931,2872,2807,2753,2697$, 1944, 1682, 1602, 1495, 1454, 1435, 1195, 1147, 1104, 1073, 1030; MS (70 ev, EI) $m / z(\%) 200\left(\mathrm{M}^{+}, 20.01\right)$; HRMS calcd for $\mathrm{C}_{14} \mathrm{H}_{16} \mathrm{O}\left[\mathrm{M}^{+}\right]$: 200.1201, found: 200.1199 .

\section{Synthesis of tetrasubstituted enals}

1. 2,3-Diethyltetradec-2(Z)-enal ((Z)-2a) (djx-3-109)

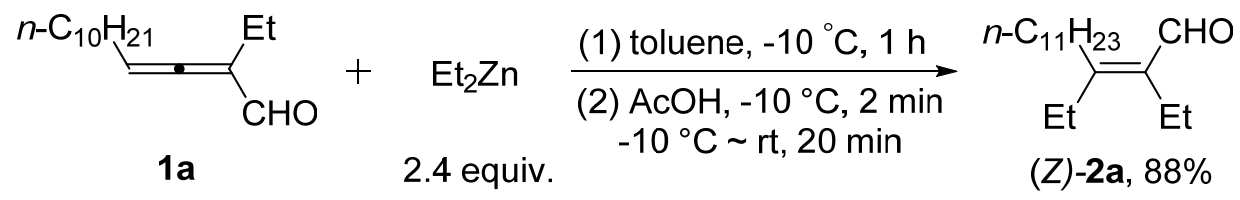

Typical Procedure II: To a dry Schlenk flask were added 1a $(0.2358 \mathrm{~g}, 1.0 \mathrm{mmol})$ and toluene $(20 \mathrm{~mL})$ under a nitrogen atmosphere. Then a solution of $\mathrm{Et}_{2} \mathrm{Zn}(1.2 \mathrm{~mL}$, 2.0 $\mathrm{M}$ in toluene, $2.4 \mathrm{mmol}$ ) was added via a syringe within 4 min with stirring at -10 ${ }^{\circ} \mathrm{C}$. After being stirred for $1 \mathrm{~h}$ at $-10{ }^{\circ} \mathrm{C}$, acetic acid $(2 \mathrm{~mL})$ was added via a syringe to the reaction mixture within $2 \mathrm{~min}$ at $-10{ }^{\circ} \mathrm{C}$ with stirring, followed by warming up to room temperature. After 20 min with stirring, ethyl acetate $(20 \mathrm{~mL})$ was added. The resulting mixture was washed sequentially with an aqueous solution of $\mathrm{HCl}(5 \%, 20$ $\mathrm{mL})$, a saturated aqueous solution of $\mathrm{NaHCO}_{3}(20 \mathrm{~mL})$, and brine $(20 \mathrm{~mL})$. The combined aqueous layer was extracted with ethyl acetate $(20 \mathrm{~mL} \times 2)$. The combined organic layer was dried over anhydrous $\mathrm{Na}_{2} \mathrm{SO}_{4}$. After filtration and evaporation, the residue was added $35 \mu \mathrm{L}$ of dibromomethane for the NMR analysis and purified by flash chromatography on silica gel (eluent: petroleum ether $\left(30-60{ }^{\circ} \mathrm{C}\right) /$ diethyl ether $=$ $100 / 1)$ to afford $(Z)-2 a(0.2339 \mathrm{~g}, 88 \%)$ as a liquid: ${ }^{1} \mathrm{H}$ NMR $\left(300 \mathrm{MHz}, \mathrm{CDCl}_{3}\right) \delta$ $10.07(\mathrm{~s}, 1 \mathrm{H}, \mathrm{CHO}), 2.53\left(\mathrm{t}, J=8.0 \mathrm{~Hz}, 2 \mathrm{H}, \mathrm{CH}_{2}\right), 2.36-2.19\left(\mathrm{~m}, 4 \mathrm{H}, \mathrm{CH}_{2} \times 2\right)$, 1.54-1.42 (m, 2H, $\left.\mathrm{CH}_{2}\right), 1.41-1.18\left(\mathrm{~m}, 16 \mathrm{H}, \mathrm{CH}_{2} \times 8\right), 1.10$ (t, J=7.7 Hz, 3H, $\mathrm{CH}_{3}$ ), $0.94\left(\mathrm{t}, J=7.5 \mathrm{~Hz}, 3 \mathrm{H}, \mathrm{CH}_{3}\right), 0.88\left(\mathrm{t}, J=6.6 \mathrm{~Hz}, 3 \mathrm{H}, \mathrm{CH}_{3}\right) ;{ }^{13} \mathrm{C}$ NMR $(75 \mathrm{MHz}$, $\left.\mathrm{CDCl}_{3}\right) \delta 191.1,163.8,137.7,31.7,30.7,30.0,29.54,29.45,29.4,29.25,29.18,27.3$, 22.5, 18.2, 13.9, 12.7; IR (neat) $v\left(\mathrm{~cm}^{-1}\right)$ 2961, 2925, 2872, 2854, 2752, 1668, 1620, 1463, 1397, 1376, 1337, 1287, 1253, 1155, 1059; MS (70 ev, EI) $m / z(\%) 266\left(\mathrm{M}^{+}\right.$, 31.47), 237 (100); HRMS calcd for $\mathrm{C}_{18} \mathrm{H}_{34} \mathrm{O}\left[\mathrm{M}^{+}\right]$: 266.2610, found: 266.2613. 


\section{2,3-Diethyltridec-2(Z)-enal ((Z)-2b) (djx-2-190)}

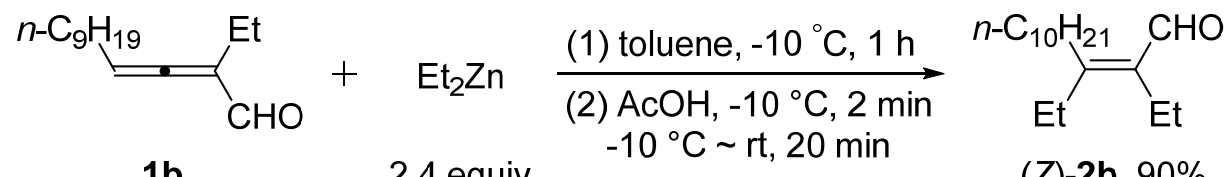

Following Typical Procedure II, the reaction of $\mathbf{1 b}(0.2221 \mathrm{~g}, 1.0 \mathrm{mmol})$, toluene $(20 \mathrm{~mL})$, a solution of $\mathrm{Et}_{2} \mathrm{Zn}(1.6 \mathrm{~mL}, 1.5 \mathrm{M}$ in toluene, $2.4 \mathrm{mmol})$, and $\mathrm{AcOH}(2 \mathrm{~mL})$ afforded (Z)-2b $(0.2273 \mathrm{~g}, 90 \%)$ (eluent: petroleum ether $\left(30-60{ }^{\circ} \mathrm{C}\right) /$ diethyl ether $=$ 100/1) as a liquid: ${ }^{1} \mathrm{H}$ NMR (300 MHz, $\left.\mathrm{CDCl}_{3}\right) \delta 10.07$ (s, 1H, CHO), 2.53 (t, $J=7.8$ $\left.\mathrm{Hz}, 2 \mathrm{H}, \mathrm{CH}_{2}\right), 2.34-2.20\left(\mathrm{~m}, 4 \mathrm{H}, \mathrm{CH}_{2} \times 2\right), 1.57-1.42\left(\mathrm{~m}, 2 \mathrm{H}, \mathrm{CH}_{2}\right), 1.41-1.19(\mathrm{~m}$, $\left.14 \mathrm{H}, \mathrm{CH}_{2} \times 7\right), 1.10\left(\mathrm{t}, J=7.5 \mathrm{~Hz}, 3 \mathrm{H}, \mathrm{CH}_{3}\right), 0.94\left(\mathrm{t}, J=7.7 \mathrm{~Hz}, 3 \mathrm{H}, \mathrm{CH}_{3}\right), 0.88(\mathrm{t}, J$ $\left.=6.8 \mathrm{~Hz}, 3 \mathrm{H}, \mathrm{CH}_{3}\right) ;{ }^{13} \mathrm{C} \mathrm{NMR}\left(75 \mathrm{MHz}, \mathrm{CDCl}_{3}\right) \delta 191.2,163.9,137.7,31.7,30.7$, $30.0,29.6,29.41,29.39,29.3,29.2,27.3,22.5,18.2,13.9,12.7$; IR (neat) $v\left(\mathrm{~cm}^{-1}\right)$ 2961, 2926, 2855, 2752, 1669, 1618, 1464, 1376, 1336, 1287, 1251, 1155, 1060; MS (70 ev, EI) $m / z(\%) 252\left(\mathrm{M}^{+}, 30.82\right), 223$ (100); HRMS calcd for $\mathrm{C}_{17} \mathrm{H}_{32} \mathrm{O}\left[\mathrm{M}^{+}\right]$: 252.2453, found: 252.2448 .

3. 3-Ethyl-2-methyltridec-2(Z)-enal ((Z)-2c) (djx-3-018)

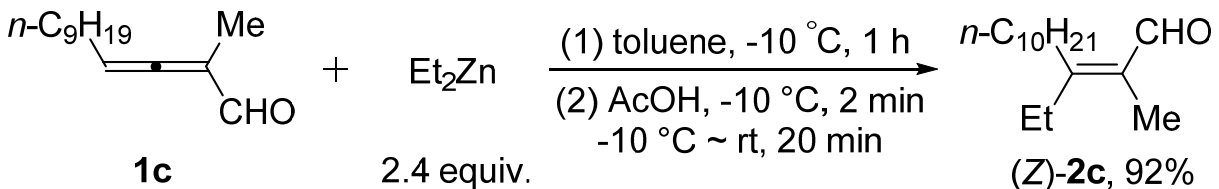

Following Typical Procedure II, the reaction of 1c (0.2076 g, $1.0 \mathrm{mmol})$, toluene $(20 \mathrm{~mL})$, a solution of $\mathrm{Et}_{2} \mathrm{Zn}(1.6 \mathrm{~mL}, 1.5 \mathrm{M}$ in toluene, $2.4 \mathrm{mmol})$, and $\mathrm{AcOH}(2 \mathrm{~mL})$ afforded (Z)-2c $(0.2181 \mathrm{~g}, 92 \%)$ (eluent: petroleum ether $\left(30-60{ }^{\circ} \mathrm{C}\right) /$ diethyl ether $=$ 100/1) as a liquid: ${ }^{1} \mathrm{H}$ NMR (300 MHz, $\left.\mathrm{CDCl}_{3}\right) \delta 10.09$ (s, 1H, CHO), 2.55 (t, $J=7.8$ $\left.\mathrm{Hz}, 2 \mathrm{H}, \mathrm{CH}_{2}\right), 2.28$ (q, $\left.J=7.5 \mathrm{~Hz}, 2 \mathrm{H}, \mathrm{CH}_{2}\right), 1.75\left(\mathrm{~s}, 3 \mathrm{H}, \mathrm{CH}_{3}\right), 1.58-1.41(\mathrm{~m}, 2 \mathrm{H}$, $\left.\mathrm{CH}_{2}\right), 1.40-1.17\left(\mathrm{~m}, 14 \mathrm{H}, \mathrm{CH}_{2} \times 7\right), 1.08\left(\mathrm{t}, J=7.7 \mathrm{~Hz}, 3 \mathrm{H}, \mathrm{CH}_{3}\right), 0.88(\mathrm{t}, J=6.8 \mathrm{~Hz}$, $\left.3 \mathrm{H}, \mathrm{CH}_{3}\right) ;{ }^{13} \mathrm{C} \mathrm{NMR}\left(75 \mathrm{MHz}, \mathrm{CDCl}_{3}\right) \delta 191.4,164.5,131.6,31.8,30.5,30.4,29.6$, 29.5, 29.4, 29.3, 29.2, 28.1, 22.6, 14.0, 11.9, 10.1; IR (neat) $v\left(\mathrm{~cm}^{-1}\right) 2957,2925$, 2855, 2752, 1671, 1623, 1467, 1396, 1377, 1325, 1282, 1156, 1029; MS (70 ev, EI) 
$m / z(\%) 238\left(\mathrm{M}^{+}, 27.08\right), 43$ (100); HRMS calcd for $\mathrm{C}_{16} \mathrm{H}_{30} \mathrm{O}\left[\mathrm{M}^{+}\right]: 238.2297$, found: 238.2297 .

4. 3-Ethyl-2-propylundec-2(Z)-enal ((Z)-2d) (djx-3-010)

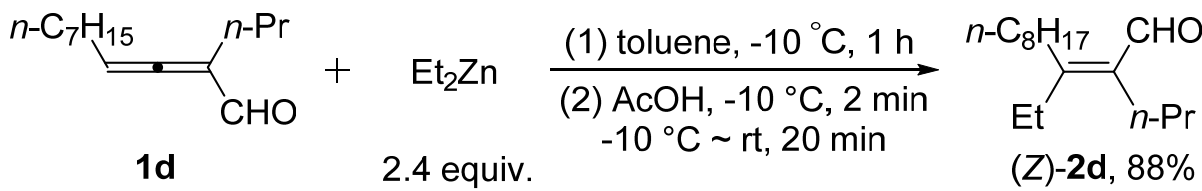

Following Typical Procedure II, the reaction of 1d (0.2078 g, $1.0 \mathrm{mmol})$, toluene $(20 \mathrm{~mL})$, a solution of $\mathrm{Et}_{2} \mathrm{Zn}(1.6 \mathrm{~mL}, 1.5 \mathrm{M}$ in toluene, $2.4 \mathrm{mmol})$, and $\mathrm{AcOH}(2 \mathrm{~mL})$ afforded (Z)-2d (0.2090 g, 88\%) (eluent: petroleum ether $\left(30-60{ }^{\circ} \mathrm{C}\right) /$ diethyl ether $=$ 100/1) as a liquid: ${ }^{1} \mathrm{H}$ NMR (300 MHz, $\left.\mathrm{CDCl}_{3}\right) \delta 10.07$ (s, 1H, CHO), 2.53 (t, $J=7.8$ $\left.\mathrm{Hz}, 2 \mathrm{H}, \mathrm{CH}_{2}\right), 2.35-2.15\left(\mathrm{~m}, 4 \mathrm{H}, \mathrm{CH}_{2} \times 2\right), 1.60-1.42\left(\mathrm{~m}, 2 \mathrm{H}, \mathrm{CH}_{2}\right), 1.41-1.18(\mathrm{~m}$, $\left.12 \mathrm{H}, \mathrm{CH}_{2} \times 6\right), 1.09\left(\mathrm{t}, J=7.5 \mathrm{~Hz}, 3 \mathrm{H}, \mathrm{CH}_{3}\right), 0.96-0.82\left(\mathrm{~m}, 6 \mathrm{H}, \mathrm{CH}_{3} \times 2\right) ;{ }^{13} \mathrm{C} \mathrm{NMR}$ $\left(75 \mathrm{MHz}, \mathrm{CDCl}_{3}\right) \delta 191.6,164.5,136.3,31.7,30.9,30.0,29.6,29.3,29.1,27.5,27.0$, 22.7, 22.5, 14.1, 14.0, 12.7; IR (neat) $v\left(\mathrm{~cm}^{-1}\right)$ 2959, 2927, 2869, 2857, 2753, 1670, 1617, 1464, 1394, 1377, 1346, 1154, 1090, 1066; MS (70 ev, EI) $m / z(\%) 238\left(\mathrm{M}^{+}\right.$, 45.52), 41 (100); HRMS calcd for $\mathrm{C}_{16} \mathrm{H}_{30} \mathrm{O}\left[\mathrm{M}^{+}\right]$: 238.2297, found: 238.2300.

5. 3-Ethyl-2-propyltridec-2(Z)-enal ((Z)-2e) (djx-3-015)

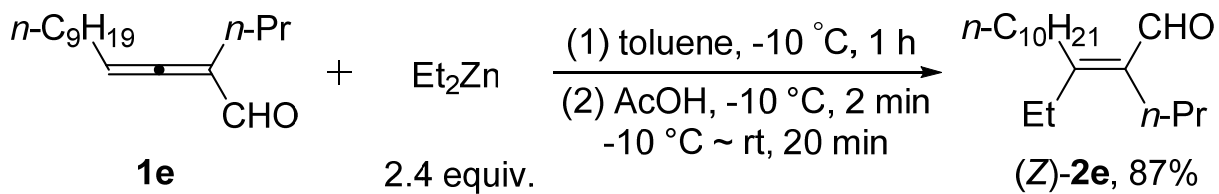

Following Typical Procedure II, the reaction of 1e $(0.2359 \mathrm{~g}, 1.0 \mathrm{mmol})$, toluene $(20 \mathrm{~mL})$, a solution of $\mathrm{Et}_{2} \mathrm{Zn}(1.6 \mathrm{~mL}, 1.5 \mathrm{M}$ in toluene, $2.4 \mathrm{mmol})$, and $\mathrm{AcOH}(2 \mathrm{~mL})$ afforded $(Z)-2 \mathbf{e}(0.2314 \mathrm{~g}, 87 \%)$ (eluent: petroleum ether $\left(30-60{ }^{\circ} \mathrm{C}\right) /$ diethyl ether $=$ 100/1) as a liquid: ${ }^{1} \mathrm{H}$ NMR (300 MHz, $\left.\mathrm{CDCl}_{3}\right) \delta 10.07$ (s, $\left.1 \mathrm{H}, \mathrm{CHO}\right), 2.53$ (t, J=7.8 $\left.\mathrm{Hz}, 2 \mathrm{H}, \mathrm{CH}_{2}\right), 2.35-2.15\left(\mathrm{~m}, 4 \mathrm{H}, \mathrm{CH}_{2} \times 2\right), 1.57-1.41\left(\mathrm{~m}, 2 \mathrm{H}, \mathrm{CH}_{2}\right), 1.40-1.19(\mathrm{~m}$, $\left.16 \mathrm{H}, \mathrm{CH}_{2} \times 8\right), 1.09\left(\mathrm{t}, J=7.7 \mathrm{~Hz}, 3 \mathrm{H}, \mathrm{CH}_{3}\right), 0.95-0.81\left(\mathrm{~m}, 6 \mathrm{H}, \mathrm{CH}_{3} \times 2\right) ;{ }^{13} \mathrm{C} \mathrm{NMR}$ $\left(75 \mathrm{MHz}, \mathrm{CDCl}_{3}\right) \delta 191.5,164.4,136.3,31.8,30.9,30.0,29.6,29.5,29.4,29.3,29.2$, 27.5, 27.0, 22.7, 22.6, 14.1, 14.0, 12.7; IR (neat) $v\left(\mathrm{~cm}^{-1}\right) 2959,2926,2855,2753$, 
1669, 1617, 1464, 1397, 1377, 1348, 1153, 1067; MS (70 ev, EI) m/z (\%) $266\left(\mathrm{M}^{+}\right.$, 35.18), 43 (100); HRMS calcd for $\mathrm{C}_{18} \mathrm{H}_{34} \mathrm{O}\left[\mathrm{M}^{+}\right]$: 266.2610, found: 266.2614.

\section{3-Ethyl-2-propyltetradec-2(Z)-enal ((Z)-2f) (djx-3-058)}

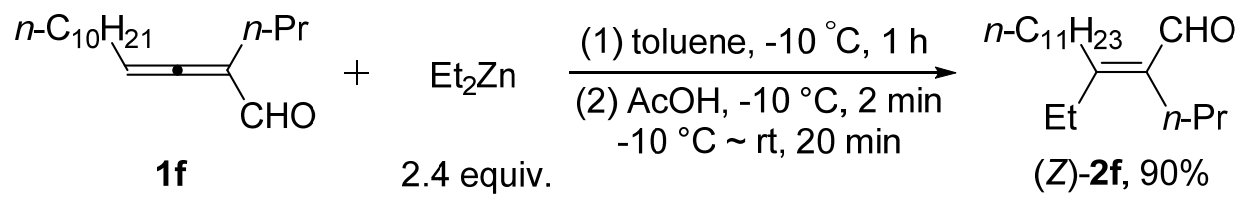

Following Typical Procedure II, the reaction of $\mathbf{1 f}(0.2501 \mathrm{~g}, 1.0 \mathrm{mmol})$, toluene $(20 \mathrm{~mL})$, a solution of $\mathrm{Et}_{2} \mathrm{Zn}(1.2 \mathrm{~mL}, 2.0 \mathrm{M}$ in toluene, $2.4 \mathrm{mmol})$, and $\mathrm{AcOH}(2 \mathrm{~mL})$ afforded $(Z)-2 f(0.2521 \mathrm{~g}, 90 \%)$ (eluent: petroleum ether $\left(30-60{ }^{\circ} \mathrm{C}\right) /$ diethyl ether $=$ 100/1) as a liquid: ${ }^{1} \mathrm{H}$ NMR (300 MHz, $\left.\mathrm{CDCl}_{3}\right) \delta 10.07$ (s, 1H, CHO), $2.53(\mathrm{t}, J=7.8$ $\left.\mathrm{Hz}, 2 \mathrm{H}, \mathrm{CH}_{2}\right), 2.35-2.15\left(\mathrm{~m}, 4 \mathrm{H}, \mathrm{CH}_{2} \times 2\right), 1.57-1.41\left(\mathrm{~m}, 2 \mathrm{H}, \mathrm{CH}_{2}\right), 1.40-1.20(\mathrm{~m}$, $\left.18 \mathrm{H}, \mathrm{CH}_{2} \times 9\right), 1.09\left(\mathrm{t}, J=7.5 \mathrm{~Hz}, 3 \mathrm{H}, \mathrm{CH}_{3}\right), 0.96-0.82\left(\mathrm{~m}, 6 \mathrm{H}, \mathrm{CH}_{3} \times 2\right) ;{ }^{13} \mathrm{C} \mathrm{NMR}$ $\left(75 \mathrm{MHz}, \mathrm{CDCl}_{3}\right) \delta 191.5,164.4,136.3,31.8,30.9,30.0,29.6,29.5,29.4,29.3,29.2$, 27.5, 27.0, 22.7, 22.6, 14.1, 14.0, 12.7; IR (neat) $v\left(\mathrm{~cm}^{-1}\right)$ 2959, 2926, 2869, 2854, $2753,1670,1618,1465,1397,1377,1349,1326,1230,1154,1067$; MS (70 ev, EI) $m / z(\%) 280\left(\mathrm{M}^{+}, 62.68\right), 237$ (100); HRMS calcd for $\mathrm{C}_{19} \mathrm{H}_{36} \mathrm{O}\left[\mathrm{M}^{+}\right]$: 280.2766, found: 280.2764 .

Five gram scale synthesis of $(Z)-2 f(d j x-3-065)$

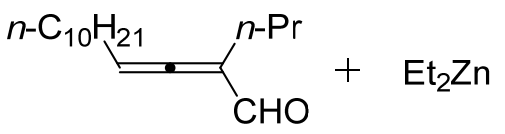

1f, $5.25 \mathrm{~g}, 21 \mathrm{mmol} \quad 2.4$ equiv. $\underset{\text { (1) toluene, }-10^{\circ} \mathrm{C}, 12 \mathrm{~h}}{\stackrel{\mathrm{AcOH},}{\mathrm{C}}-10^{\circ} \mathrm{C}, 20 \mathrm{~min}}$ $-10^{\circ} \mathrm{C} \sim \mathrm{rt}, 3 \mathrm{~h}$

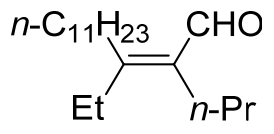

(Z)-2f, 93\%, $5.45 \mathrm{~g}$

To a dry three-neck flask (1 L) were added 1f $(5.2502 \mathrm{~g}, 21 \mathrm{mmol})$ and toluene $(420 \mathrm{~mL})$ under a nitrogen atmosphere. Then a solution of $\mathrm{Et}_{2} \mathrm{Zn}(25.2 \mathrm{~mL}, 2.0 \mathrm{M}$ in toluene, $50.4 \mathrm{mmol}$ ) was added dropwise within $20 \mathrm{~min}$ with stirring at $-10{ }^{\circ} \mathrm{C}$. After being stirred for $12 \mathrm{~h}$ at $-10{ }^{\circ} \mathrm{C}$, acetic acid $(42 \mathrm{~mL})$ was added dropwise to the reaction mixture within $20 \mathrm{~min}$ at $-10{ }^{\circ} \mathrm{C}$, followed by warming up to room temperature with stirring. After $3 \mathrm{~h}$, ethyl acetate $(200 \mathrm{~mL})$ was added. The resulting mixture was washed sequentially with an aqueous solution of $\mathrm{HCl}(5 \%, 200 \mathrm{~mL})$, a saturated aqueous solution of $\mathrm{NaHCO}_{3}(200 \mathrm{~mL})$, and brine $(200 \mathrm{~mL})$. The combined 
aqueous layer was extracted with ethyl acetate $(50 \mathrm{~mL} \times 2)$. The combined organic layer was dried over anhydrous $\mathrm{Na}_{2} \mathrm{SO}_{4}$. After filtration and evaporation, the residue was purified by flash chromatography on silica gel (eluent: petroleum ether (30-60 $\left.{ }^{\circ} \mathrm{C}\right) /$ diethyl ether $\left.=100 / 1\right)$ to afford $(Z)-2 f(5.4479 \mathrm{~g}, 93 \%)$ as a liquid: ${ }^{1} \mathrm{H}$ NMR (300 $\left.\mathrm{MHz}, \mathrm{CDCl}_{3}\right) \delta 10.07(\mathrm{~s}, 1 \mathrm{H}, \mathrm{CHO}), 2.53\left(\mathrm{t}, J=8.0 \mathrm{~Hz}, 2 \mathrm{H}, \mathrm{CH}_{2}\right), 2.37-2.15(\mathrm{~m}, 4 \mathrm{H}$, $\left.\mathrm{CH}_{2} \times 2\right), 1.59-1.18\left(\mathrm{~m}, 20 \mathrm{H}, \mathrm{CH}_{2} \times 10\right), 1.09\left(\mathrm{t}, J=7.5 \mathrm{~Hz}, 3 \mathrm{H}, \mathrm{CH}_{3}\right), 0.96-0.83(\mathrm{~m}$, $\left.6 \mathrm{H}, \mathrm{CH}_{3} \times 2\right) .{ }^{13} \mathrm{C} \mathrm{NMR}\left(75 \mathrm{MHz}, \mathrm{CDCl}_{3}\right) \delta 191.6,164.5,136.4,31.9,30.9,30.1$, 29.7, 29.6, 29.5, 29.4, 29.3, 27.6, 27.1, 22.8, 22.7, 14.2, 14.1, 12.7.

7. 3-(Cyclohexylmethyl)-2-propylpent-2(Z)-enal ((Z)-2g) (djx-2-198)

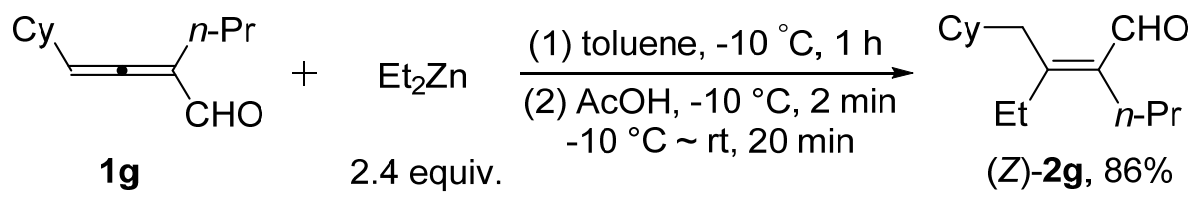

Following Typical Procedure II, the reaction of $1 \mathrm{~g}(0.1921 \mathrm{~g}, 1.0 \mathrm{mmol})$, toluene $(20 \mathrm{~mL})$, a solution of $\mathrm{Et}_{2} \mathrm{Zn}(1.6 \mathrm{~mL}, 1.5 \mathrm{M}$ in toluene, $2.4 \mathrm{mmol})$, and $\mathrm{AcOH}(2 \mathrm{~mL})$ afforded $(Z)-2 \mathrm{~g}(0.1911 \mathrm{~g}, 86 \%)$ (eluent: petroleum ether $\left(30-60{ }^{\circ} \mathrm{C}\right) /$ diethyl ether $=$ 100/1) as a liquid: ${ }^{1} \mathrm{H}$ NMR (300 MHz, $\left.\mathrm{CDCl}_{3}\right) \delta 10.05$ (s, 1H, CHO), 2.45 (d, J= 7.2 $\left.\mathrm{Hz}, 2 \mathrm{H}, \mathrm{CH}_{2}\right), 2.34-2.17\left(\mathrm{~m}, 4 \mathrm{H}, \mathrm{CH}_{2} \times 2\right), 1.81-1.59\left(\mathrm{~m}, 5 \mathrm{H}, \mathrm{CH}\right.$ and $\left.\mathrm{CH}_{2} \times 2\right)$, 1.55-1.13 (m, 6H, $\left.\mathrm{CH}_{2} \times 3\right), 1.08\left(\mathrm{t}, J=7.5 \mathrm{~Hz}, 3 \mathrm{H}, \mathrm{CH}_{3}\right), 1.03-0.85\left(\mathrm{~m}, 5 \mathrm{H}, \mathrm{CH}_{2}\right.$ and $\left.\mathrm{CH}_{3}\right) ;{ }^{13} \mathrm{C} \mathrm{NMR}\left(75 \mathrm{MHz}, \mathrm{CDCl}_{3}\right) \delta 191.4,162.6,137.6,38.2,36.9,33.2,27.5,27.1$, 26.1, 22.7, 14.1, 12.6; IR (neat) $v\left(\mathrm{~cm}^{-1}\right)$ 2960, 2926, 2853, 2755, 1668, 1615, 1449, 1397, 1376, 1349, 1324, 1293, 1269, 1154, 1093, 1068, 1038; MS (70 ev, EI) m/z (\%) $222\left(\mathrm{M}^{+}, 23.11\right), 55$ (100); HRMS calcd for $\mathrm{C}_{15} \mathrm{H}_{26} \mathrm{O}\left[\mathrm{M}^{+}\right]$: 222.1984, found: 222.1986 .

8. 3-Ethyl-5-phenyl-2-propylpent-2(Z)-enal ((Z)-2h) (djx-3-063)

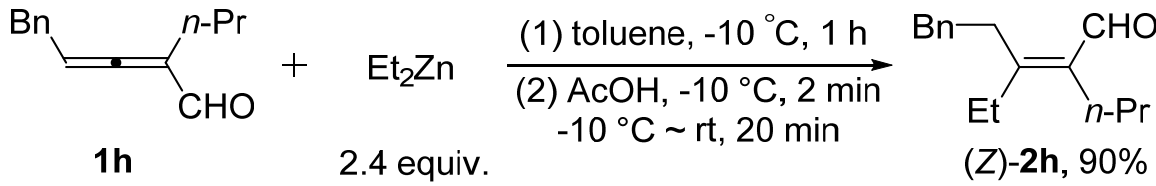

Following Typical Procedure II, the reaction of $\mathbf{1 h}(0.1997 \mathrm{~g}, 1.0 \mathrm{mmol})$, toluene 
$(20 \mathrm{~mL})$, a solution of $\mathrm{Et}_{2} \mathrm{Zn}(1.2 \mathrm{~mL}, 2.0 \mathrm{M}$ in toluene, $2.4 \mathrm{mmol})$, and $\mathrm{AcOH}(2 \mathrm{~mL})$ afforded $(Z)-2 \mathbf{h}(0.2068 \mathrm{~g}, 90 \%)$ (eluent: petroleum ether $\left(30-60{ }^{\circ} \mathrm{C}\right) /$ diethyl ether $=$ 50/1) as a liquid: ${ }^{1} \mathrm{H} \mathrm{NMR}\left(300 \mathrm{MHz}, \mathrm{CDCl}_{3}\right) \delta 9.86(\mathrm{~s}, 1 \mathrm{H}, \mathrm{CHO}), 7.31-7.23(\mathrm{~m}, 2 \mathrm{H}$, ArH), 7.22-7.08 (m, 3H, ArH), 2.88-2.73 (m, 4H, $\left.\mathrm{CH}_{2} \times 2\right), 2.28$ (q, J=7.7 Hz, 2H, $\left.\mathrm{CH}_{2}\right), 2.23-2.14\left(\mathrm{~m}, 2 \mathrm{H}, \mathrm{CH}_{2}\right), 1.38-1.23\left(\mathrm{~m}, 2 \mathrm{H}, \mathrm{CH}_{2}\right), 1.10\left(\mathrm{t}, J=7.5 \mathrm{~Hz}, 3 \mathrm{H}, \mathrm{CH}_{3}\right)$, $0.90\left(\mathrm{t}, J=7.4 \mathrm{~Hz}, 3 \mathrm{H}, \mathrm{CH}_{3}\right) ;{ }^{13} \mathrm{C} \mathrm{NMR}\left(75 \mathrm{MHz}, \mathrm{CDCl}_{3}\right) \delta 191.1,162.0,140.3$, $136.9,128.4,128.3,126.2,36.5,31.8,27.3,27.1,22.6,14.2,12.6$; IR (neat) $v\left(\mathrm{~cm}^{-1}\right)$ $3085,3062,3027,2962,2933,2872,2756,1667,1615,1496,1464,1454,1397,1377$, 1347, 1323, 1292, 1152, 1075; MS (70 ev, EI) m/z (\%) $230\left(\mathrm{M}^{+}, 1.05\right), 91$ (100); HRMS calcd for $\mathrm{C}_{16} \mathrm{H}_{22} \mathrm{O}\left[\mathrm{M}^{+}\right]: 230.1671$, found: 230.1673 .

9. 7-Chloro-3-ethyl-2-propylhept-2(Z)-enal ((Z)-2i) (djx-3-050)

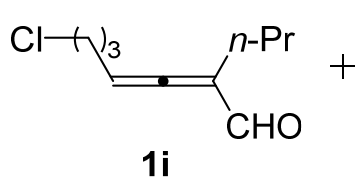

$1 \mathbf{i}$
$\mathrm{Et}_{2} \mathrm{Zn}$

2.4 equiv. $\underset{(1) \text { toluene, }-10^{\circ} \mathrm{C}, 1 \mathrm{~h}}{\mathrm{(2)} \mathrm{AcOH},-10^{\circ} \mathrm{C}, 2 \mathrm{~min}}$
$-10^{\circ} \mathrm{C} \sim \mathrm{rt}, 20 \mathrm{~min}$

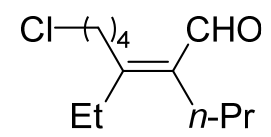

(Z)-2i, $87 \%$

Following Typical Procedure II, the reaction of $1 \mathbf{i}(0.1870 \mathrm{~g}, 1.0 \mathrm{mmol})$, toluene $(20 \mathrm{~mL})$, a solution of $\mathrm{Et}_{2} \mathrm{Zn}(1.6 \mathrm{~mL}, 1.5 \mathrm{M}$ in toluene, $2.4 \mathrm{mmol})$, and $\mathrm{AcOH}(2 \mathrm{~mL})$ afforded (Z)-2i $(0.1890 \mathrm{~g}, 87 \%)$ (eluent: petroleum ether $\left(30-60{ }^{\circ} \mathrm{C}\right) /$ diethyl ether $=$ 20/1) as a liquid: ${ }^{1} \mathrm{H}$ NMR $\left(300 \mathrm{MHz}, \mathrm{CDCl}_{3}\right) \delta 10.07$ (s, $\left.1 \mathrm{H}, \mathrm{CHO}\right), 3.57$ (t, $J=6.3$ $\left.\mathrm{Hz}, 2 \mathrm{H}, \mathrm{CH}_{2}\right), 2.58\left(\mathrm{t}, J=8.0 \mathrm{~Hz}, 2 \mathrm{H}, \mathrm{CH}_{2}\right), 2.37-2.16\left(\mathrm{~m}, 4 \mathrm{H}, \mathrm{CH}_{2} \times 2\right), 1.90-1.77$ (m, 2H, $\mathrm{CH}_{2}$ ), 1.74-1.60 (m, 2H, $\left.\mathrm{CH}_{2}\right), 1.40-1.24\left(\mathrm{~m}, 2 \mathrm{H}, \mathrm{CH}_{2}\right), 1.11$ (t, J=7.5 Hz, $\left.3 \mathrm{H}, \mathrm{CH}_{3}\right), 0.91\left(\mathrm{t}, J=7.4 \mathrm{~Hz}, 3 \mathrm{H}, \mathrm{CH}_{3}\right) ;{ }^{13} \mathrm{C} \mathrm{NMR}\left(75 \mathrm{MHz}, \mathrm{CDCl}_{3}\right) \delta 191.3,163.2$, 136.8, 44.4, 32.2, 29.1, 27.8, 27.4, 27.1, 22.7, 14.2, 12.7; IR (neat) $v\left(\mathrm{~cm}^{-1}\right)$ 2961, 2932, 2871, 2756, 1667, 1616, 1462, 1456, 1399, 1377, 1348, 1301, 1225, 1154, 1067; MS (70 ev, EI) m/z (\%) $218\left(\mathrm{M}^{+}\left({ }^{37} \mathrm{Cl}\right), 17.40\right), 216\left(\mathrm{M}^{+}\left({ }^{35} \mathrm{Cl}\right), 48.51\right), 55(100)$; HRMS calcd for $\mathrm{C}_{12} \mathrm{H}_{21} \mathrm{O}^{35} \mathrm{Cl}\left[\mathrm{M}^{+}\right]$: 216.1281, found: 216.1279 .

10. 3-Ethyl-2-propyltrideca-2(Z),12-dienal ((Z)-2j) (djx-3-031) 


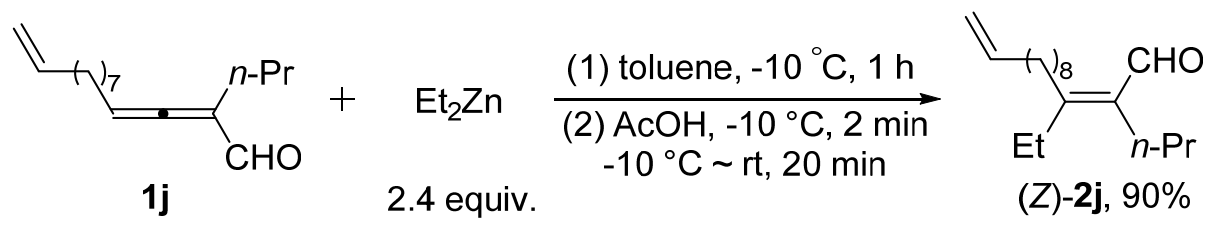

Following Typical Procedure II, the reaction of $\mathbf{1 j}(0.2338$ g, $1.0 \mathrm{mmol}$, purity: $96 \%$ ), toluene $(20 \mathrm{~mL})$, a solution of $\mathrm{Et}_{2} \mathrm{Zn}(1.2 \mathrm{~mL}, 2.0 \mathrm{M}$ in toluene, $2.4 \mathrm{mmol})$, and AcOH (2 mL) afforded (Z)-2j (0.2374 g, 90\%, purity: 96\%) (eluent: petroleum ether $\left(30-60{ }^{\circ} \mathrm{C}\right) /$ diethyl ether $\left.=100 / 1\right)$ as a liquid: ${ }^{1} \mathrm{H} \mathrm{NMR}\left(300 \mathrm{MHz}, \mathrm{CDCl}_{3}\right) \delta 10.07(\mathrm{~s}$, $1 \mathrm{H}, \mathrm{CHO}), 5.90-5.72(\mathrm{~m}, 1 \mathrm{H},=\mathrm{CH}), 5.05-4.88\left(\mathrm{~m}, 2 \mathrm{H},=\mathrm{CH}_{2}\right), 2.53(\mathrm{t}, J=7.8 \mathrm{~Hz}, 2 \mathrm{H}$, $\left.\mathrm{CH}_{2}\right), 2.36-2.15\left(\mathrm{~m}, 4 \mathrm{H}, \mathrm{CH}_{2} \times 2\right), 2.04\left(\mathrm{q}, 2 \mathrm{H}, J=7.0 \mathrm{~Hz}, \mathrm{CH}_{2}\right), 1.57-1.20(\mathrm{~m}, 14 \mathrm{H}$, $\left.\mathrm{CH}_{2} \times 7\right), 1.09\left(\mathrm{t}, J=7.7 \mathrm{~Hz}, 3 \mathrm{H}, \mathrm{CH}_{3}\right), 0.90\left(\mathrm{t}, J=7.4 \mathrm{~Hz}, 3 \mathrm{H}, \mathrm{CH}_{3}\right) ;{ }^{13} \mathrm{C} \mathrm{NMR}(75$ $\left.\mathrm{MHz}, \mathrm{CDCl}_{3}\right) \delta 191.5,164.4,139.0,136.3,114.1,33.7,30.9,30.0,29.6,29.2,28.9$, 28.8, 27.5, 27.0, 22.7, 14.1, 12.7; IR (neat) $v\left(\mathrm{~cm}^{-1}\right)$ 3076, 2962, 2928, 2856, 2754, 1735, 1669, 1641, 1617, 1464, 1376, 1291, 1229, 1153; MS (70 ev, EI) m/z (\%) 264 $\left(\mathrm{M}^{+}, 20.23\right), 55$ (100); HRMS calcd for $\mathrm{C}_{18} \mathrm{H}_{32} \mathrm{O}\left[\mathrm{M}^{+}\right]$: 264.2453, found: 264.2451.

\section{3-(4-methylbenzyl)-2-propylpent-2(Z)-enal ((Z)-2k) (djx-3-186)}

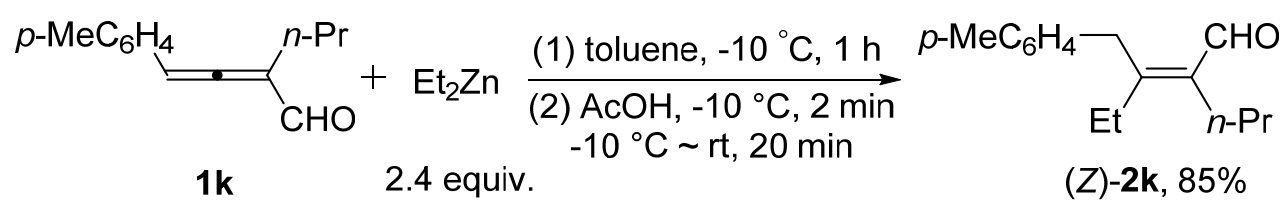

Following Typical Procedure II, the reaction of $\mathbf{1 k}(0.1999$ g, $1.0 \mathrm{mmol})$, toluene $(20 \mathrm{~mL})$, a solution of $\mathrm{Et}_{2} \mathrm{Zn}(1.2 \mathrm{~mL}, 2.0 \mathrm{M}$ in toluene, $2.4 \mathrm{mmol})$, and $\mathrm{AcOH}(2 \mathrm{~mL})$ afforded $(Z)-2 \mathbf{k}(0.1956 \mathrm{~g}, 85 \%)$ (eluent: petroleum ether $\left(30-60{ }^{\circ} \mathrm{C}\right) /$ diethyl ether $=$ 100/1) as a liquid: ${ }^{1} \mathrm{H}$ NMR (300 $\left.\mathrm{MHz}, \mathrm{CDCl}_{3}\right) \delta 10.18(\mathrm{~s}, 1 \mathrm{H}, \mathrm{CHO}), 7.11(\mathrm{~d}, J=7.8$ $\mathrm{Hz}, 2 \mathrm{H}, \mathrm{ArH}), 7.04$ (d, $J=8.1 \mathrm{~Hz}, 2 \mathrm{H}, \mathrm{ArH}), 3.90$ (s, 2H, $\left.\mathrm{CH}_{2}\right), 2.38-2.17$ (m, 7H, $\mathrm{CH}_{3}$ and $\left.\mathrm{CH}_{2} \times 2\right), 1.48-1.31\left(\mathrm{~m}, 2 \mathrm{H}, \mathrm{CH}_{2}\right), 1.06\left(\mathrm{t}, J=7.5 \mathrm{~Hz}, 3 \mathrm{H}, \mathrm{CH}_{3}\right), 0.94(\mathrm{t}, J=$ $\left.7.4 \mathrm{~Hz}, 3 \mathrm{H}, \mathrm{CH}_{3}\right) ;{ }^{13} \mathrm{C} \mathrm{NMR}\left(75 \mathrm{MHz}, \mathrm{CDCl}_{3}\right) \delta$ 192.0, 161.1, 137.9, 136.2, 135.4, 129.3, 128.3, 34.6, 27.4, 27.0, 22.8, 20.9, 14.3, 12.7; IR (neat) $v\left(\mathrm{~cm}^{-1}\right)$ 3048, 3021, 2962, 2932, 2871, 2755, 1668, 1619, 1513, 1463, 1399, 1377, 1346, 1326, 1294, 1150, 1117, 1108, 1067, 1045; MS (70 ev, EI) m/z (\%) $230\left(\mathrm{M}^{+}, 19.69\right), 187$ (100); HRMS calcd for $\mathrm{C}_{16} \mathrm{H}_{22} \mathrm{O}\left[\mathrm{M}^{+}\right]$: 230.1671, found: 230.1666 . 
12. 3-Benzyl-2-butylpent-2(Z)-enal ((Z)-2l) (djx-2-193)

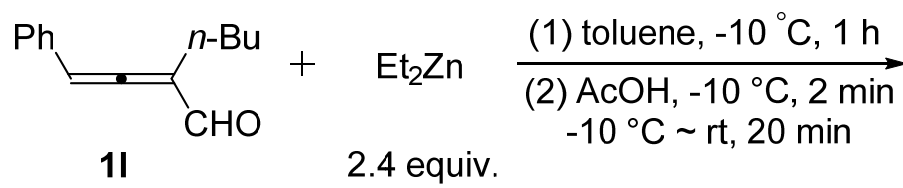<smiles>CCC(C=O)=C(C=O)Cc1ccccc1</smiles>

(Z)-2I, $90 \%$

Following Typical Procedure II, the reaction of $\mathbf{1 l}(0.2003 \mathrm{~g}, 1.0 \mathrm{mmol})$, toluene $(20 \mathrm{~mL})$, a solution of $\mathrm{Et}_{2} \mathrm{Zn}$ in toluene $(1.6 \mathrm{~mL}, 1.5 \mathrm{M}, 2.4 \mathrm{mmol})$, and $\mathrm{AcOH}(2 \mathrm{~mL})$ afforded $(Z)-2 \mathrm{l}(0.2063 \mathrm{~g}, 90 \%)$ (eluent: petroleum ether $\left(30-60{ }^{\circ} \mathrm{C}\right) /$ diethyl ether $=$ 100/1) as a liquid: ${ }^{1} \mathrm{H}$ NMR $\left(300 \mathrm{MHz}, \mathrm{CDCl}_{3}\right) \delta 10.17(\mathrm{~s}, 1 \mathrm{H}, \mathrm{CHO}), 7.35-7.10(\mathrm{~m}$, 5H, ArH), $3.92\left(\mathrm{~s}, 2 \mathrm{H}, \mathrm{CH}_{2}\right), 2.33\left(\mathrm{t}, J=7.5 \mathrm{~Hz}, 2 \mathrm{H}, \mathrm{CH}_{2}\right), 2.22(\mathrm{q}, J=7.6 \mathrm{~Hz}, 2 \mathrm{H}$, $\mathrm{CH}_{2}$ ), 1.45-1.28 (m, 4H, $\left.\mathrm{CH}_{2} \times 2\right), 1.05$ (t, $\left.J=7.5 \mathrm{~Hz}, 3 \mathrm{H}, \mathrm{CH}_{3}\right), 0.92$ (t, $J=6.9 \mathrm{~Hz}$, $\left.3 \mathrm{H}, \mathrm{CH}_{3}\right) ;{ }^{13} \mathrm{C}$ NMR $\left(75 \mathrm{MHz}, \mathrm{CDCl}_{3}\right) \delta 191.5,160.2,138.3,138.1,128.5,128.3$, 126.4, 34.8, 31.6, 26.9, 25.0, 22.8, 13.8, 12.5; IR (neat) $v\left(\mathrm{~cm}^{-1}\right) 3062,3027,2959$, 2932, 2872, 2756, 1668, 1617, 1601, 1495, 1453, 1399, 1377, 1277, 1151, 1072, 1028; MS (70 ev, EI) $m / z(\%) 230\left(\mathrm{M}^{+}, 17.38\right), 145$ (100); HRMS calcd for $\mathrm{C}_{16} \mathrm{H}_{22} \mathrm{O}\left[\mathrm{M}^{+}\right]$: 230.1671, found: 230.1670 .

13. 2-Butyl-3-(4-methylbenzyl)pent-2(Z)-enal ((Z)-2m) (djx-3-145)

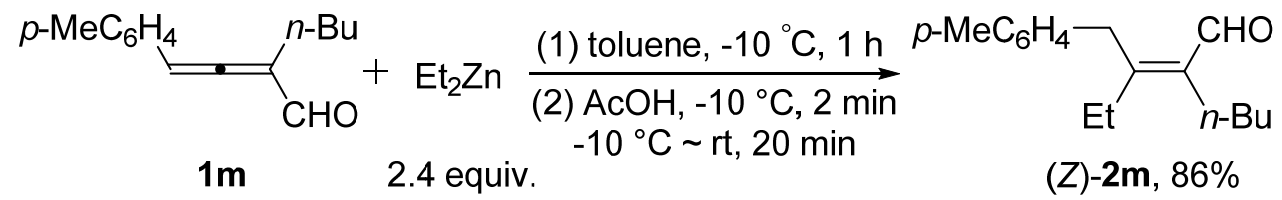

Following Typical Procedure II, the reaction of $\mathbf{1 m}(0.2141 \mathrm{~g}, 1.0 \mathrm{mmol})$, toluene $(20 \mathrm{~mL})$, a solution of $\mathrm{Et}_{2} \mathrm{Zn}$ in toluene $(1.2 \mathrm{~mL}, 2.0 \mathrm{M}, 2.4 \mathrm{mmol})$, and $\mathrm{AcOH}(2 \mathrm{~mL})$ afforded $(Z)-2 \mathbf{m}(0.2098 \mathrm{~g}, 86 \%)$ (eluent: petroleum ether $\left(30-60{ }^{\circ} \mathrm{C}\right) /$ diethyl ether $=$ 100/1) as a liquid: ${ }^{1} \mathrm{H}$ NMR $\left(300 \mathrm{MHz}, \mathrm{CDCl}_{3}\right) \delta 10.18(\mathrm{~s}, 1 \mathrm{H}, \mathrm{CHO}), 7.10(\mathrm{~d}, J=8.1$ $\mathrm{Hz}, 2 \mathrm{H}, \mathrm{ArH}), 7.03$ (d, $J=7.8 \mathrm{~Hz}, 2 \mathrm{H}, \mathrm{ArH}), 3.89$ (s, 2H, $\left.\mathrm{CH}_{2}\right), 2.37-2.27$ (m, 5H, $\mathrm{CH}_{3}$ and $\left.\mathrm{CH}_{2}\right), 2.22\left(\mathrm{q}, J=7.6 \mathrm{~Hz}, 2 \mathrm{H}, \mathrm{CH}_{2}\right), 1.44-1.27\left(\mathrm{~m}, 4 \mathrm{H}, \mathrm{CH}_{2} \times 2\right), 1.06(\mathrm{t}, J=$ $\left.7.7 \mathrm{~Hz}, 3 \mathrm{H}, \mathrm{CH}_{3}\right), 0.92\left(\mathrm{t}, J=6.8 \mathrm{~Hz}, 3 \mathrm{H}, \mathrm{CH}_{3}\right) ;{ }^{13} \mathrm{C} \mathrm{NMR}\left(75 \mathrm{MHz}, \mathrm{CDCl}_{3}\right) \delta 191.8$, $160.8,138.1,136.1,135.3,129.3,128.3,34.5,31.7,26.9,25.1,23.0,20.8,13.9,12.6$; IR (neat) $v\left(\mathrm{~cm}^{-1}\right) 3048,3021,2958,2931,2872,2757,1667,1620,1513,1460,1398$, $1377,1335,1278,1185,1151,1118,1105,1072,1043,1021 ;$ MS (70 ev, EI) $m / z(\%)$ 
$244\left(\mathrm{M}^{+}, 16.09\right), 187$ (100); HRMS calcd for $\mathrm{C}_{17} \mathrm{H}_{24} \mathrm{O}\left[\mathrm{M}^{+}\right]$: 244.1827, found: 244.1829 .

14. 2-Allyl-3-ethyltridec-2(Z)-enal ((Z)-2n) (djx-3-068)

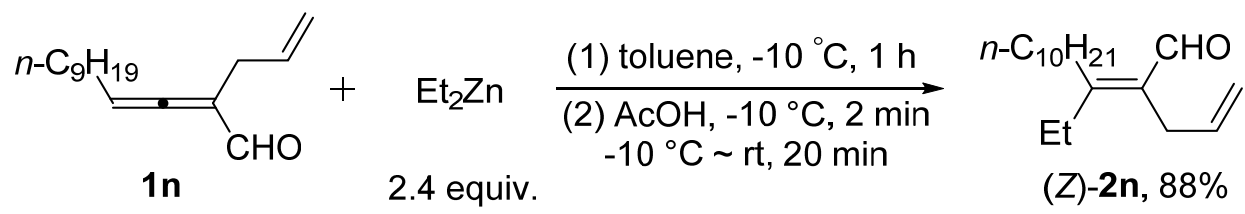

Following Typical Procedure II, the reaction of 1n (0.2343 g, $1.0 \mathrm{mmol})$, toluene $(20 \mathrm{~mL})$, a solution of $\mathrm{Et}_{2} \mathrm{Zn}(1.6 \mathrm{~mL}, 1.5 \mathrm{M}$ in toluene, $2.4 \mathrm{mmol})$, and $\mathrm{AcOH}(2 \mathrm{~mL})$ afforded (Z)-2n (0.2324 g, 88\%) (eluent: petroleum ether $\left(30-60{ }^{\circ} \mathrm{C}\right) /$ diethyl ether $=$ 100/1) as a liquid: ${ }^{1} \mathrm{H}$ NMR $\left(300 \mathrm{MHz}, \mathrm{CDCl}_{3}\right) \delta 10.09$ (s, 1H, CHO), 5.85-5.68 (m, $1 \mathrm{H},=\mathrm{CH}), 4.99-4.87\left(\mathrm{~m}, 2 \mathrm{H},=\mathrm{CH}_{2}\right), 3.02\left(\mathrm{dd}, J_{l}=5.7 \mathrm{~Hz}, J_{2}=0.9 \mathrm{~Hz}, 2 \mathrm{H}, \mathrm{CH}_{2}\right)$, $2.58\left(\mathrm{t}, J=8.0 \mathrm{~Hz}, 2 \mathrm{H}, \mathrm{CH}_{2}\right), 2.29$ (q, $\left.J=7.4 \mathrm{~Hz}, 2 \mathrm{H}, \mathrm{CH}_{2}\right), 1.60-1.45\left(\mathrm{~m}, 2 \mathrm{H}, \mathrm{CH}_{2}\right)$, 1.43-1.18 (m, 14H, $\left.\mathrm{CH}_{2} \times 7\right), 1.09\left(\mathrm{t}, J=7.7 \mathrm{~Hz}, 3 \mathrm{H}, \mathrm{CH}_{3}\right), 0.88$ (t, $J=6.2 \mathrm{~Hz}, 3 \mathrm{H}$, $\left.\mathrm{CH}_{3}\right) ;{ }^{13} \mathrm{C} \mathrm{NMR}\left(75 \mathrm{MHz}, \mathrm{CDCl}_{3}\right) \delta 190.6,165.8,135.8,133.4,114.3,31.7,30.7$, $30.2,29.6,29.40,29.36,29.2,29.1,28.8,27.6,22.5,13.9,12.3$; IR (neat) $v\left(\mathrm{~cm}^{-1}\right)$ 3079, 2957, 2925, 2855, 2752, 1671, 1638, 1619, 1467, 1396, 1378, 1328, 1153, 1068; MS (70 ev, EI) m/z (\%) $264\left(\mathrm{M}^{+}, 6.56\right), 123$ (100); HRMS calcd for $\mathrm{C}_{18} \mathrm{H}_{32} \mathrm{O}$ $\left[\mathrm{M}^{+}\right]: 264.2453$, found: 264.2452 .

15. 2-Benzyl-3-ethylpent-2-enal (2o) (djx-3-021)<smiles>CC=C=C(C=O)CBr</smiles>

10

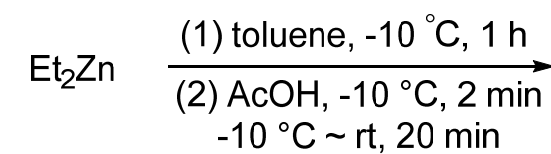

2.4 equiv.

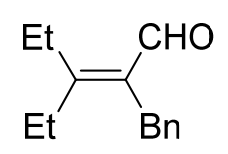

2o, $88 \%$

Following Typical Procedure II, the reaction of $10(0.1718 \mathrm{~g}, 1.0 \mathrm{mmol})$, toluene $(20 \mathrm{~mL})$, a solution of $\mathrm{Et}_{2} \mathrm{Zn}(1.6 \mathrm{~mL}, 1.5 \mathrm{M}$ in toluene, $2.4 \mathrm{mmol})$, and $\mathrm{AcOH}(2 \mathrm{~mL})$ afforded $20(0.1777 \mathrm{~g}, 88 \%)$ (eluent: petroleum ether $\left(30-60{ }^{\circ} \mathrm{C}\right) /$ diethyl ether $\left.=50 / 1\right)$ as a liquid: ${ }^{1} \mathrm{H}$ NMR $\left(300 \mathrm{MHz}, \mathrm{CDCl}_{3}\right) \delta 10.18(\mathrm{~s}, 1 \mathrm{H}, \mathrm{CHO}), 7.27-7.18(\mathrm{~m}, 2 \mathrm{H}$, ArH), 7.17-7.07 (m, 3H, ArH), 3.65 (s, 2H, $\left.\mathrm{CH}_{2}\right), 2.64$ (q, J=7.6 Hz, 2H, $\left.\mathrm{CH}_{2}\right), 2.31$ (q, $\left.J=7.5 \mathrm{~Hz}, 2 \mathrm{H}, \mathrm{CH}_{2}\right), 1.19\left(\mathrm{t}, J=7.7 \mathrm{~Hz}, 3 \mathrm{H}, \mathrm{CH}_{3}\right), 0.99$ (t, $\left.J=7.5 \mathrm{~Hz}, 3 \mathrm{H}, \mathrm{CH}_{3}\right)$; 
${ }^{13} \mathrm{C}$ NMR $\left(75 \mathrm{MHz}, \mathrm{CDCl}_{3}\right) \delta$ 191.2, 167.8, 140.2, 134.3, 128.2, 127.9, 125.6, 30.1, 27.7, 23.3, 15.3, 12.1; IR (neat) $v\left(\mathrm{~cm}^{-1}\right)$ 3084, 3061, 3027, 2972, 2935, 2875, 2755, 1667, 1617, 1494, 1453, 1378, 1328, 1281, 1246, 1153, 1074, 1042, 1030; MS (70 ev, EI) $m / z(\%) 202\left(\mathrm{M}^{+}, 59.88\right), 91(100)$; HRMS calcd for $\mathrm{C}_{14} \mathrm{H}_{18} \mathrm{O}\left[\mathrm{M}^{+}\right]$: 202.1358, found: 202.1360 .

16. 3-Butyl-2-propylundec-2(Z)-enal ((Z)-2p) (djx-3-027)

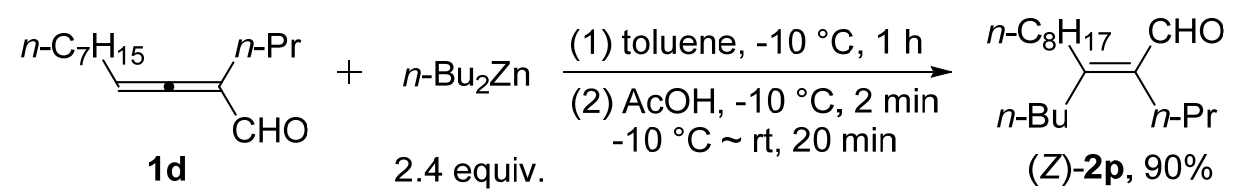

Following Typical Procedure II, the reaction of $1 \mathbf{d}(0.2079$ g, $1.0 \mathrm{mmol})$, toluene $(20 \mathrm{~mL})$, a solution of $n-\mathrm{Bu}_{2} \mathrm{Zn}$ in toluene $(2.4 \mathrm{~mL}, 1.0 \mathrm{M}, 2.4 \mathrm{mmol})$, and AcOH (2 $\mathrm{mL})$ afforded $(Z)-2 \mathbf{p}(0.2387 \mathrm{~g}, 90 \%)$ (eluent: petroleum ether $\left(30-60{ }^{\circ} \mathrm{C}\right) /$ diethyl ether $=100 / 1)$ as a liquid: ${ }^{1} \mathrm{H}$ NMR $\left(300 \mathrm{MHz}, \mathrm{CDCl}_{3}\right) \delta 10.07(\mathrm{~s}, 1 \mathrm{H}, \mathrm{CHO}), 2.52(\mathrm{t}$, $\left.J=7.8 \mathrm{~Hz}, 2 \mathrm{H}, \mathrm{CH}_{2}\right), 2.33-2.14\left(\mathrm{~m}, 4 \mathrm{H}, \mathrm{CH}_{2} \times 2\right), 1.57-1.17\left(\mathrm{~m}, 18 \mathrm{H}, \mathrm{CH}_{2} \times 9\right)$, 1.00-0.83 (m, 9H, $\left.\mathrm{CH}_{3} \times 3\right) ;{ }^{13} \mathrm{C} \mathrm{NMR}\left(75 \mathrm{MHz}, \mathrm{CDCl}_{3}\right) \delta 191.6,163.5,136.6,34.5$, 31.8, 31.0, 30.6, 29.7, 29.3, 29.1, 27.2, 23.1, 22.7, 22.6, 14.2, 14.0, 13.9; IR (neat) v $\left(\mathrm{cm}^{-1}\right) 2958,2927,2858,2753,1668,1616,1465,1397,1378,1348,1154,1083 ; \mathrm{MS}$ (70 ev, EI) $m / z(\%) 266\left(\mathrm{M}^{+}, 37.72\right), 126(100)$; HRMS calcd for $\mathrm{C}_{18} \mathrm{H}_{34} \mathrm{O}\left[\mathrm{M}^{+}\right]$: 266.2610, found: 266.2611 .

17. 3-Butyl-2-propyltetradec-2(Z)-enal ((Z)-2q) (djx-3-046)

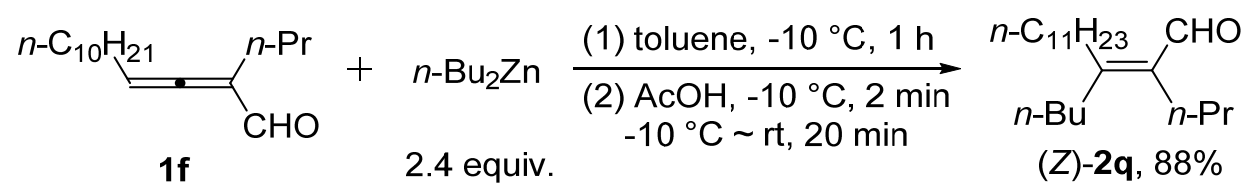

Following Typical Procedure II, the reaction of $\mathbf{1 f}(0.2502 \mathrm{~g}, 1.0 \mathrm{mmol})$, toluene $(20 \mathrm{~mL})$, a solution of $n-\mathrm{Bu}_{2} \mathrm{Zn}(2.4 \mathrm{~mL}, 1.0 \mathrm{M}$ in toluene, $2.4 \mathrm{mmol})$, and $\mathrm{AcOH}(2$ $\mathrm{mL})$ afforded $(Z)-2 q(0.2710 \mathrm{~g}, 88 \%)$ (eluent: petroleum ether $\left(30-60{ }^{\circ} \mathrm{C}\right) /$ diethyl ether $=200 / 1)$ as a liquid: ${ }^{1} \mathrm{H}$ NMR $\left(300 \mathrm{MHz}, \mathrm{CDCl}_{3}\right) \delta 10.07(\mathrm{~s}, 1 \mathrm{H}, \mathrm{CHO}), 2.53(\mathrm{t}$, $\left.J=8.0 \mathrm{~Hz}, 2 \mathrm{H}, \mathrm{CH}_{2}\right), 2.31-2.15\left(\mathrm{~m}, 4 \mathrm{H}, \mathrm{CH}_{2} \times 2\right), 1.57-1.21\left(\mathrm{~m}, 24 \mathrm{H}, \mathrm{CH}_{2} \times 12\right)$, 
1.00-0.83 (m, 9H, $\left.\mathrm{CH}_{3} \times 3\right) ;{ }^{13} \mathrm{C}$ NMR $\left(75 \mathrm{MHz} \mathrm{CDCl}_{3}\right) \delta 191.5,163.4,136.6,34.5$, $31.8,31.0,30.57,30.55,29.6,29.52,29.45,29.32,29.26,27.2,23.1,22.7,22.6,14.2$, 14.0, 13.8; IR (neat) $v\left(\mathrm{~cm}^{-1}\right)$ 2958, 2926, 2855, 2752, 1670, 1615, 1464, 1397, 1378, 1348, 1311, 1271, 1229, 1152, 1081; MS (70 ev, EI) m/z (\%) $308\left(\mathrm{M}^{+}, 25.06\right), 43$ (100); HRMS calcd for $\mathrm{C}_{21} \mathrm{H}_{40} \mathrm{O}\left[\mathrm{M}^{+}\right]$: 308.3079 , found: 308.3080 .

18. 3-Phenethyl-2-propylhept-2(Z)-enal ((Z)-2r) (djx-3-062)

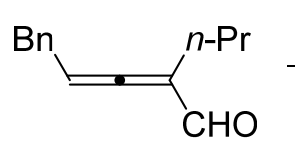

$1 \mathrm{~h}$

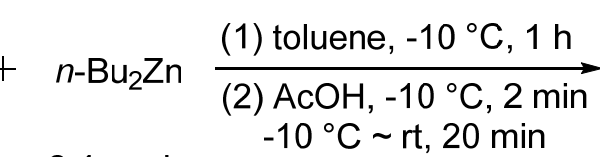

2.4 equiv.

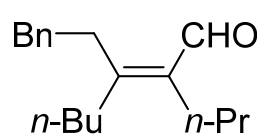

(Z)-2r, $89 \%$

Following Typical Procedure II, the reaction of $\mathbf{1 h}(0.1998$ g, $1.0 \mathrm{mmol})$, toluene (20 mL), a solution of $n-\mathrm{Bu}_{2} \mathrm{Zn}(2.4 \mathrm{~mL}, 1.0 \mathrm{M}$ in toluene, $2.4 \mathrm{mmol})$, and $\mathrm{AcOH}(2$ $\mathrm{mL})$ afforded $(Z)-2 \mathbf{r}(0.2296 \mathrm{~g}, 89 \%)$ (eluent: petroleum ether $\left(30-60{ }^{\circ} \mathrm{C}\right) /$ diethyl ether $=50 / 1)$ as a liquid: ${ }^{1} \mathrm{H}$ NMR $\left(300 \mathrm{MHz}, \mathrm{CDCl}_{3}\right) \delta 9.87(\mathrm{~s}, 1 \mathrm{H}, \mathrm{CHO}), 7.31-7.23(\mathrm{~m}$, 2H, ArH), 7.23-7.09 (m, 3H, ArH), 2.88-2.73 (m, 4H, $\left.\mathrm{CH}_{2} \times 2\right), 2.31-2.13(\mathrm{~m}, 4 \mathrm{H}$, $\left.\mathrm{CH}_{2} \times 2\right), 1.54-1.21\left(\mathrm{~m}, 6 \mathrm{H}, \mathrm{CH}_{2} \times 3\right), 1.10-0.85\left(\mathrm{~m}, 6 \mathrm{H}, \mathrm{CH}_{3} \times 2\right) ;{ }^{13} \mathrm{C}$ NMR $(75$ $\left.\mathrm{MHz}, \mathrm{CDCl}_{3}\right) \delta 191.1,160.9,140.4,137.3,128.4,128.3,126.3,36.6,34.2,32.3,30.5$, 27.3, 23.0, 22.6, 14.2, 13.8; IR (neat) $v\left(\mathrm{~cm}^{-1}\right) 3085,3063,3027,2959,2931,2871$, 2756, 1668, 1614, 1496, 1464, 1454, 1397, 1379, 1379, 1347, 1150, 1097, 1075, 1031; MS (70 ev, EI) m/z (\%) $258\left(\mathrm{M}^{+}, 4.46\right), 91$ (100); HRMS calcd for $\mathrm{C}_{18} \mathrm{H}_{26} \mathrm{O}\left[\mathrm{M}^{+}\right]$: 258.1984, found: 258.1985 .

19. 3-Butyl-7-chloro-2-propylhept-2(Z)-enal ((Z)-2s) (djx-3-051)

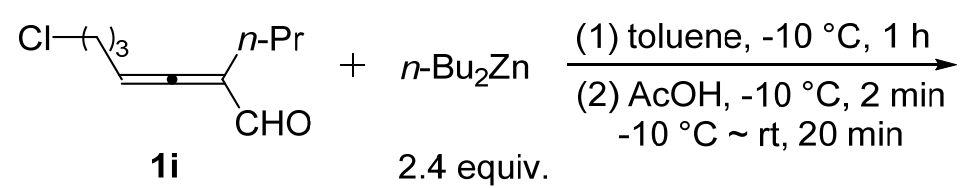

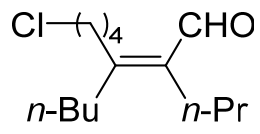

(Z)-2s, $86 \%$

Following Typical Procedure II, the reaction of 1i (0.1865 g, $1.0 \mathrm{mmol})$, toluene (20 mL), a solution of $n-\mathrm{Bu}_{2} \mathrm{Zn}(2.4 \mathrm{~mL}, 1.0 \mathrm{M}$ in toluene, $2.4 \mathrm{mmol})$, and $\mathrm{AcOH}(2$ $\mathrm{mL})$ afforded $(Z)-2 \mathrm{~s}(0.2107 \mathrm{~g}, 86 \%)$ (eluent: petroleum ether $\left(30-60{ }^{\circ} \mathrm{C}\right) /$ diethyl ether $=30 / 1)$ as a liquid: ${ }^{1} \mathrm{H}$ NMR $\left(300 \mathrm{MHz}, \mathrm{CDCl}_{3}\right) \delta 10.07(\mathrm{~s}, 1 \mathrm{H}, \mathrm{CHO}), 3.56(\mathrm{t}, J=6.3$ 
$\left.\mathrm{Hz}, 2 \mathrm{H}, \mathrm{CH}_{2}\right), 2.58\left(\mathrm{t}, J=8.0 \mathrm{~Hz}, 2 \mathrm{H}, \mathrm{CH}_{2}\right), 2.32-2.14\left(\mathrm{~m}, 4 \mathrm{H}, \mathrm{CH}_{2} \times 2\right), 1.90-1.76$ (m, 2H, $\left.\mathrm{CH}_{2}\right), 1.74-1.59\left(\mathrm{~m}, 2 \mathrm{H}, \mathrm{CH}_{2}\right), 1.52-1.23\left(\mathrm{~m}, 6 \mathrm{H}, \mathrm{CH}_{2} \times 3\right), 1.00-0.85(\mathrm{~m}, 6 \mathrm{H}$, $\left.\mathrm{CH}_{3} \times 2\right) ;{ }^{13} \mathrm{C} \mathrm{NMR}\left(75 \mathrm{MHz}, \mathrm{CDCl}_{3}\right) \delta 191.2,162.0,137.0,44.4,34.3,32.1,30.5$, 29.6, 27.8, 27.2, 23.0, 22.6, 14.1, 13.8; IR (neat) $v\left(\mathrm{~cm}^{-1}\right)$ 2959, 2929, 2871, 2756, 1667, 1616, 1464, 1399, 1378, 1347, 1311, 1269, 1228, 1153, 1080; MS (70 ev, EI) $m / z(\%) 246\left(\mathrm{M}^{+}\left({ }^{37} \mathrm{Cl}\right), 8.05\right), 244\left(\mathrm{M}^{+}\left({ }^{35} \mathrm{Cl}\right), 24.25\right), 55$ (100); HRMS calcd for $\mathrm{C}_{14} \mathrm{H}_{25} \mathrm{O}^{35} \mathrm{Cl}\left[\mathrm{M}^{+}\right]: 244.1594$, found: 244.1597 .

20. 2-Allyl-3-butyltridec-2(Z)-enal ((Z)-2t) (djx-3-069)

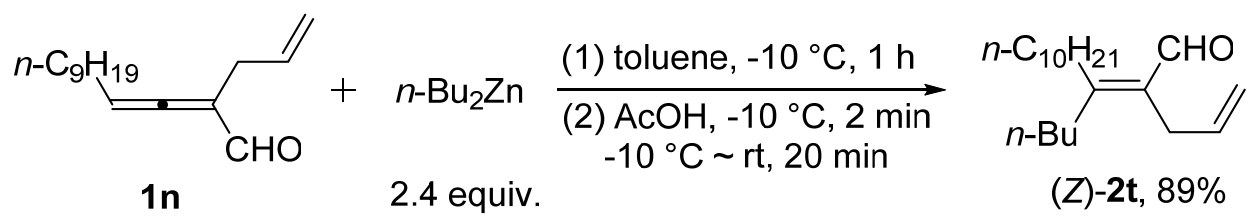

Following Typical Procedure II, the reaction of 1 n $(0.2341 \mathrm{~g}, 1.0 \mathrm{mmol})$, toluene $(20 \mathrm{~mL})$, a solution of $n-\mathrm{Bu}_{2} \mathrm{Zn}$ in toluene $(2.4 \mathrm{~mL}, 1.0 \mathrm{M}, 2.4 \mathrm{mmol})$, and $\mathrm{AcOH}(2$ $\mathrm{mL})$ afforded $(Z)-2 \mathrm{t}(0.2598 \mathrm{~g}, 89 \%)$ (eluent: petroleum ether $\left(30-60{ }^{\circ} \mathrm{C}\right) /$ diethyl ether $=100 / 1)$ as a liquid: ${ }^{1} \mathrm{H}$ NMR $\left(300 \mathrm{MHz}, \mathrm{CDCl}_{3}\right) \delta 10.08(\mathrm{~s}, 1 \mathrm{H}, \mathrm{CHO}), 5.84-5.68(\mathrm{~m}$, $1 \mathrm{H},=\mathrm{CH}), 4.99-4.87\left(\mathrm{~m}, 2 \mathrm{H},=\mathrm{CH}_{2}\right), 3.02\left(\mathrm{dt}, J_{1}=5.9 \mathrm{~Hz}, J_{2}=1.6 \mathrm{~Hz}, 2 \mathrm{H}, \mathrm{CH}_{2}\right)$, $2.57\left(\mathrm{t}, J=8.0 \mathrm{~Hz}, 2 \mathrm{H}, \mathrm{CH}_{2}\right), 2.25\left(\mathrm{t}, J=7.8 \mathrm{~Hz}, 2 \mathrm{H}, \mathrm{CH}_{2}\right), 1.60-1.15\left(\mathrm{~m}, 20 \mathrm{H}, \mathrm{CH}_{2} \times\right.$ 10), 0.99-0.81 (m, 6H, $\left.\left.\mathrm{CH}_{3} \times 2\right) ;{ }^{13} \mathrm{C} \mathrm{NMR} \mathrm{(75} \mathrm{MHz,} \mathrm{CDCl}_{3}\right) \delta 190.8,165.0,135.9$, 133.8, 114.4, 34.6, 31.8, 30.9, 30.8, 30.3, 29.7, 29.5, 29.4, 29.3, 29.2, 29.0, 23.1, 22.6, 14.0, 13.8; IR (neat) $v\left(\mathrm{~cm}^{-1}\right) 3079,2957,2926,2856,2752,1669,1638,1617,1466$, 1379, 1330, 1153, 1098; MS (70 ev, EI) m/z (\%) $292\left(\mathrm{M}^{+}, 8.02\right), 41$ (100); HRMS calcd for $\mathrm{C}_{20} \mathrm{H}_{36} \mathrm{O}\left[\mathrm{M}^{+}\right]$: 292.2766, found: 292.2769 .

\section{3-(Cyclohexylmethyl)-4-methyl-2-propylpent-2(E)-enal ((E)-2u) (djx-3-043)}

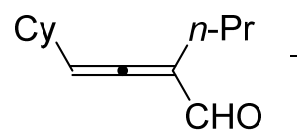

$1 \mathrm{~g}$<smiles>[R17]P</smiles>

2.4 equiv.

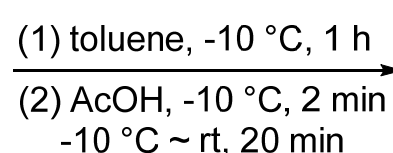
$-10^{\circ} \mathrm{C} \sim \mathrm{rt}, 20 \mathrm{~min}$

Following Typical Procedure II, the reaction of $1 \mathrm{~g}(0.1922 \mathrm{~g}, 1.0 \mathrm{mmol})$, toluene $(20 \mathrm{~mL})$, a solution of $i-\operatorname{Pr}_{2} \mathrm{Zn}(2.4 \mathrm{~mL}, 1.0 \mathrm{M}$ in toluene, $2.4 \mathrm{mmol})$, and $\mathrm{AcOH}(2$ 
$\mathrm{mL})$ afforded $(E)-2 \mathbf{u}(0.1980 \mathrm{~g}, 84 \%)$ (eluent: petroleum ether $\left(30-60{ }^{\circ} \mathrm{C}\right) /$ diethyl ether $=100 / 1)$ as a liquid: ${ }^{1} \mathrm{H}$ NMR $\left(300 \mathrm{MHz}, \mathrm{CDCl}_{3}\right) \delta 10.05(\mathrm{~s}, 1 \mathrm{H}, \mathrm{CHO}), 3.04$ (heptet, $J=7.0 \mathrm{~Hz}, 1 \mathrm{H}, \mathrm{CH}), 2.41$ (d, $J=7.2 \mathrm{~Hz}, 2 \mathrm{H}, \mathrm{CH}_{2}$ ), 2.32-2.22 (m, 2H, $\mathrm{CH}_{2}$ ), 1.78-1.59 (m, 5H, CH and $\left.\mathrm{CH}_{2} \times 2\right), 1.51-1.04\left(\mathrm{~m}, 12 \mathrm{H}, \mathrm{CH}_{2} \times 3\right.$ and $\left.\mathrm{CH}_{3} \times 2\right)$, 1.01-0.84 (m, 5H, $\mathrm{CH}_{3}$ and $\left.\mathrm{CH}_{2}\right) ;{ }^{13} \mathrm{C}$ NMR (75 MHz, $\left.\mathrm{CDCl}_{3}\right) \delta 192.6,165.0,137.5$, 39.6, 33.6, 33.5, 32.6, 27.2, 26.4, 26.2, 23.0, 21.0, 14.2; IR (neat) $v\left(\mathrm{~cm}^{-1}\right)$ 2961, 2927, 2872, 2852, 2759, 1667, 1601, 1462, 1449, 1397, 1384, 1364, 1349, 1313, 1267, 1183, 1148, 1087, 1035; MS (70 ev, EI) m/z (\%) $236\left(\mathrm{M}^{+}, 15.61\right), 193$ (100); HRMS calcd for $\mathrm{C}_{16} \mathrm{H}_{28} \mathrm{O}\left[\mathrm{M}^{+}\right]$: 236.2140, found: 236.2138.

\section{7-Chloro-3-phenyl-2-propylhept-2(E)-enal ((E)-2v) (djx-3-157)}

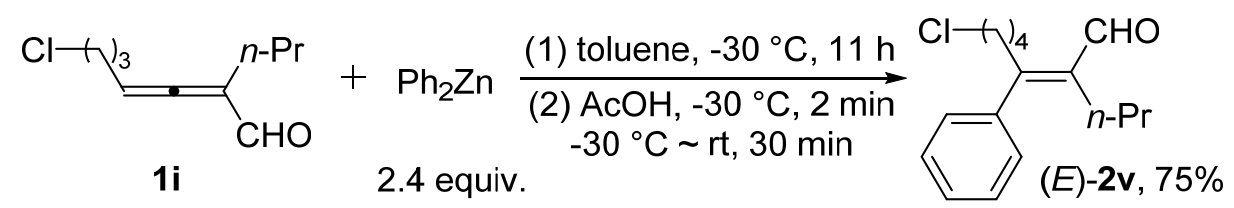

Typical Procedure III: To a dry Schlenk flask $(100 \mathrm{~mL})$ were added 1i $(0.1869$ g, $1.0 \mathrm{mmol})$ and toluene $(20 \mathrm{~mL})$ under a nitrogen atmosphere. Then a suspension of $\mathrm{Ph}_{2} \mathrm{Zn}(0.5391 \mathrm{~g}, 2.4 \mathrm{mmol}, 98 \%)$ in toluene $(20 \mathrm{~mL})$ was added via a syringe within 4 min with stirring at $-30{ }^{\circ} \mathrm{C}$. After being stirred for $11 \mathrm{~h}$ at $-30{ }^{\circ} \mathrm{C}$, acetic acid $(2 \mathrm{~mL})$ was added via a syringe to the reaction mixture within 2 min at $-30{ }^{\circ} \mathrm{C}$, followed by warming up to room temperature with stirring. After $30 \mathrm{~min}$, ethyl acetate $(20 \mathrm{~mL})$ was added. The resulting mixture was washed sequentially with an aqueous solution of $\mathrm{HCl}(5 \%, 20 \mathrm{~mL})$, a saturated aqueous solution of $\mathrm{NaHCO}_{3}(20 \mathrm{~mL})$, and brine $(20$ $\mathrm{mL})$. The combined aqueous layer was extracted with ethyl acetate $(20 \mathrm{~mL} \times 2)$. The combined organic layer was dried over anhydrous $\mathrm{Na}_{2} \mathrm{SO}_{4}$. After filtration and evaporation, the residue was added $35 \mu \mathrm{L}$ of dibromomethane for the NMR analysis and purified by flash chromatography on silica gel to afford $(E)-2 \mathbf{v}(0.1982 \mathrm{~g}, 75 \%)$ (eluent: petroleum ether $\left(30-60{ }^{\circ} \mathrm{C}\right) /$ diethyl ether $\left.=30 / 1\right)$ as a liquid: ${ }^{1} \mathrm{H}$ NMR $(300$ $\left.\mathrm{MHz}, \mathrm{CDCl}_{3}\right) \delta 10.27$ (s, 1H, CHO), 7.46-7.29 (m, 3H, ArH), 7.17-7.09 (m, 2H, ArH), $3.48\left(\mathrm{t}, J=6.5 \mathrm{~Hz}, 2 \mathrm{H}, \mathrm{CH}_{2}\right), 2.89$ (t, $\left.J=7.7 \mathrm{~Hz}, 2 \mathrm{H}, \mathrm{CH}_{2}\right), 2.09-1.98\left(\mathrm{~m}, 2 \mathrm{H}, \mathrm{CH}_{2}\right)$, 1.85-1.71 (m, 2H, $\left.\mathrm{CH}_{2}\right), 1.64-1.49\left(\mathrm{~m}, 2 \mathrm{H}, \mathrm{CH}_{2}\right), 1.33-1.17\left(\mathrm{~m}, 2 \mathrm{H}, \mathrm{CH}_{2}\right), 0.71(\mathrm{t}, J=$ 
$\left.7.4 \mathrm{~Hz}, 3 \mathrm{H}, \mathrm{CH}_{3}\right) ;{ }^{13} \mathrm{C} \mathrm{NMR}\left(75 \mathrm{MHz}, \mathrm{CDCl}_{3}\right) \delta 191.6,159.5,141.1,138.3,128.3$, 127.6, 126.9, 44.3, 32.04, 31.95, 29.0, 26.1, 22.5, 14.1; IR (neat) $v\left(\mathrm{~cm}^{-1}\right) 3078,3058$, 3019, 2960, 2931, 2869, 2752, 1668, 1611, 1596, 1490, 1463, 1442, 1394, 1346, 1319, 1274, 1199, 1106, 1074, 1026; MS (70 ev, EI) $m / z(\%) 266\left(\mathrm{M}^{+}\left({ }^{37} \mathrm{Cl}\right), 20.77\right), 264$ $\left(\mathrm{M}^{+}\left({ }^{35} \mathrm{Cl}\right), 62.47\right), 173$ (100); HRMS calcd for $\mathrm{C}_{16} \mathrm{H}_{21} \mathrm{O}^{35} \mathrm{Cl}\left[\mathrm{M}^{+}\right]$: 264.1281, found: 264.1277 .

\section{2-Butyl-3-phenyl-4-(p-tolyl)but-2(E)-enal ((E)-2w) (djx-3-150)}

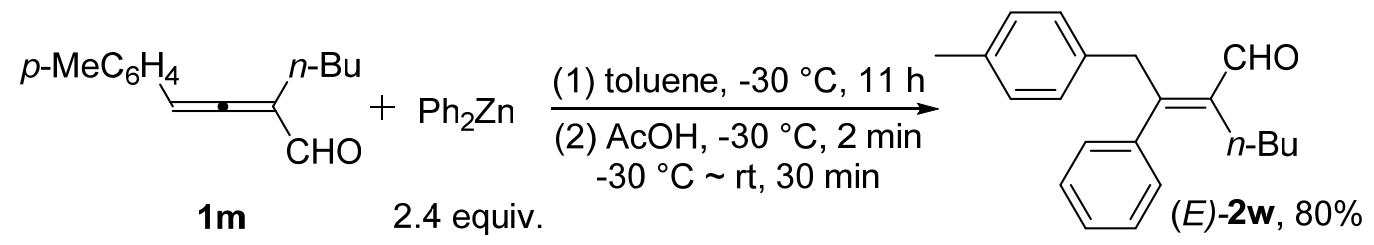

Following Typical Procedure III, the reaction of $1 \mathrm{~m}(0.2138 \mathrm{~g}, 1.0 \mathrm{mmol})$, toluene $(20 \mathrm{~mL})$, a suspension of $\mathrm{Ph}_{2} \mathrm{Zn}(0.5387 \mathrm{~g}, 2.4 \mathrm{mmol}, 98 \%)$ in toluene (20 $\mathrm{mL})$, and $\mathrm{AcOH}(2 \mathrm{~mL})$ afforded $(E)-2 \mathbf{x}(0.2334 \mathrm{~g}, 80 \%)$ (eluent: petroleum ether $\left(30-60{ }^{\circ} \mathrm{C}\right) /$ diethyl ether $\left.=50 / 1\right)$ as a liquid: ${ }^{1} \mathrm{H}$ NMR $\left(300 \mathrm{MHz}, \mathrm{CDCl}_{3}\right) \delta 10.41(\mathrm{~s}$, 1H, CHO), 7.32-7.18 (m, 3H, ArH), 7.06-6.87 (m, 6H, ArH), 4.13 (s, 2H, $\left.\mathrm{CH}_{2}\right), 2.26$ (s, 3H, $\left.\mathrm{CH}_{3}\right), 2.13\left(\mathrm{t}, J=7.8 \mathrm{~Hz}, 2 \mathrm{H}, \mathrm{CH}_{2}\right), 1.33-1.06\left(\mathrm{~m}, 4 \mathrm{H}, \mathrm{CH}_{2} \times 2\right), 0.72$ (t, $J=$ $\left.7.2 \mathrm{~Hz}, 3 \mathrm{H}, \mathrm{CH}_{3}\right) ;{ }^{13} \mathrm{C} \mathrm{NMR}\left(75 \mathrm{MHz}, \mathrm{CDCl}_{3}\right) \delta 192.1,157.5,141.1,138.9,136.0$, 134.3, 129.1, 128.5, 128.0, 127.5, 127.1, 38.1, 31.5, 26.7, 22.6, 20.9, 13.6; IR (neat) $v$ $\left(\mathrm{cm}^{-1}\right)$ 3078, 3051, 3021, 3000, 2957, 2928, 2860, 2825, 2751, 1674, 1667, 1614, $1596,1575,1513,1489,1455,1442,1394,1379,1334,1308,1186,1108,1075$, 1023; MS (70 ev, EI) m/z (\%) $293\left(\mathrm{M}^{+}+1,5.98\right), 292\left(\mathrm{M}^{+}, 27.01\right), 235$ (100); HRMS calcd for $\mathrm{C}_{21} \mathrm{H}_{24} \mathrm{O}\left[\mathrm{M}^{+}\right]$: 292.1827, found: 292.1825 .

\section{2-Benzyl-3-ethylhept-2(Z)-enal ((Z)-2x) (djx-3-034)}

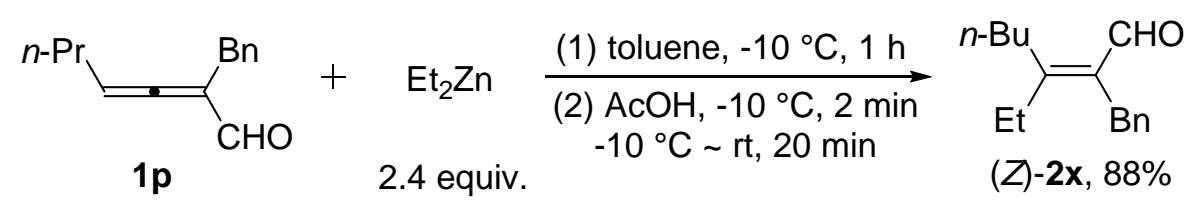

Following Typical Procedure II, the reaction of $\mathbf{1 p}(0.2001 \mathrm{~g}, 1.0 \mathrm{mmol})$, toluene $(20 \mathrm{~mL})$, a solution of $\mathrm{Et}_{2} \mathrm{Zn}(1.6 \mathrm{~mL}, 1.5 \mathrm{M}$ in toluene, $2.4 \mathrm{mmol})$, and $\mathrm{AcOH}(2 \mathrm{~mL})$ 
afforded (Z)-2x (0.2015 g, 88\%) (eluent: petroleum ether $\left(30-60{ }^{\circ} \mathrm{C}\right) /$ diethyl ether $=$ 50/1) as a liquid: ${ }^{1} \mathrm{H}$ NMR $\left(300 \mathrm{MHz}, \mathrm{CDCl}_{3}\right) \delta 10.18(\mathrm{~s}, 1 \mathrm{H}, \mathrm{CHO}), 7.28-7.04(\mathrm{~m}$, $5 \mathrm{H}, \mathrm{ArH}), 3.65$ (s, 2H, $\mathrm{CH}_{2}$ ), 2.60 (t, $\left.J=7.8 \mathrm{~Hz}, 2 \mathrm{H}, \mathrm{CH}_{2}\right), 2.30$ (q, J=7.7 Hz, 2H, $\left.\mathrm{CH}_{2}\right), 1.60-1.30\left(\mathrm{~m}, 4 \mathrm{H}, \mathrm{CH}_{2} \times 2\right), 1.04-0.87\left(\mathrm{~m}, 6 \mathrm{H}, \mathrm{CH}_{3} \times 2\right) ;{ }^{13} \mathrm{C} \mathrm{NMR}(75 \mathrm{MHz}$, $\left.\mathrm{CDCl}_{3}\right) \delta 191.1,166.4,140.2,134.8,128.2,127.8,125.6,33.0,30.2,29.9,28.0,22.8$, 13.7, 12.1; IR (neat) $v\left(\mathrm{~cm}^{-1}\right) 3062,3027,2961,2932,2873,2750,1668,1615,1495$, 1453, 1378, 1282, 1152, 1074, 1030; MS (70 ev, EI) m/z (\%) $230\left(\mathrm{M}^{+}, 43.53\right), 91$ (100); HRMS calcd for $\mathrm{C}_{16} \mathrm{H}_{22} \mathrm{O}\left[\mathrm{M}^{+}\right]$: 230.1671, found: 230.1671 .

25. 2-Benzyl-3-ethylhept-2(E)-enal ((E)-2x) (djx-4-041)

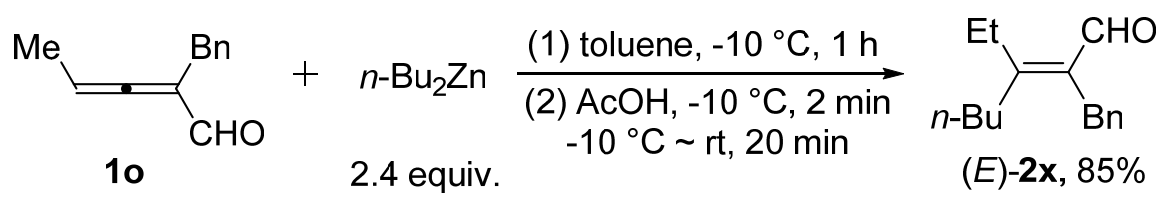

Following Typical Procedure II, the reaction of $10(0.1721 \mathrm{~g}, 1.0 \mathrm{mmol})$, toluene $(20 \mathrm{~mL})$, a solution of $n-\mathrm{Bu}_{2} \mathrm{Zn}$ in toluene $(2.4 \mathrm{~mL}, 1.0 \mathrm{M}, 2.4 \mathrm{mmol})$, and $\mathrm{AcOH}(2$ $\mathrm{mL})$ afforded $(E)-2 \mathbf{x}(0.1954 \mathrm{~g}, 85 \%)$ (eluent: petroleum ether $\left(30-60{ }^{\circ} \mathrm{C}\right) /$ diethyl ether $=100 / 1)$ as a liquid: ${ }^{1} \mathrm{H}$ NMR $\left(300 \mathrm{MHz}, \mathrm{CDCl}_{3}\right) \delta 10.18(\mathrm{~s}, 1 \mathrm{H}, \mathrm{CHO})$, 7.27-7.17 (m, 2H, ArH), 7.16-7.05 (m, 3H, ArH), 3.64 (s, 2H, $\left.\mathrm{CH}_{2}\right), 2.62$ (q, $J=7.6$ $\left.\mathrm{Hz}, 2 \mathrm{H}, \mathrm{CH}_{2}\right), 2.36-2.19\left(\mathrm{~m}, 2 \mathrm{H}, \mathrm{CH}_{2}\right), 1.39-1.23\left(\mathrm{~m}, 4 \mathrm{H}, \mathrm{CH}_{2} \times 2\right), 1.18(\mathrm{t}, J=7.7$ $\left.\mathrm{Hz}, 3 \mathrm{H}, \mathrm{CH}_{3}\right), 0.87$ (t, $\left.J=6.9 \mathrm{~Hz}, 3 \mathrm{H}, \mathrm{CH}_{3}\right) ;{ }^{13} \mathrm{C} \mathrm{NMR}\left(75 \mathrm{MHz}, \mathrm{CDCl}_{3}\right) \delta 191.0$, $166.7,140.3,134.6,128.1,127.8,125.6,34.7,30.3,29.9,23.8,23.0,15.3,13.7$; IR (neat) $v\left(\mathrm{~cm}^{-1}\right) 3084,3061,3027,2961,2932,2873,2754,1667,1615,1494,1453$, 1398, 1378, 1340, 1329, 1280, 1206, 1152, 1074, 1030; MS (70 ev, EI) m/z (\%) 230 $\left(\mathrm{M}^{+}, 35.57\right), 91$ (100); HRMS calcd for $\mathrm{C}_{16} \mathrm{H}_{22} \mathrm{O}\left[\mathrm{M}^{+}\right]$: 230.1671, found: 230.1671 . 
Table S1. The reaction of $1 \mathrm{a}$ with $\mathrm{Et}_{2} \mathrm{Zn}$ quenched with carboxylic acids bearing different $\mathrm{p} K_{\mathrm{a}}$ values under the optimized conditions.

\begin{tabular}{|c|c|c|c|c|c|}
\hline \multirow{3}{*}{ entry } & \multirow{2}{*}{\multicolumn{3}{|c|}{ 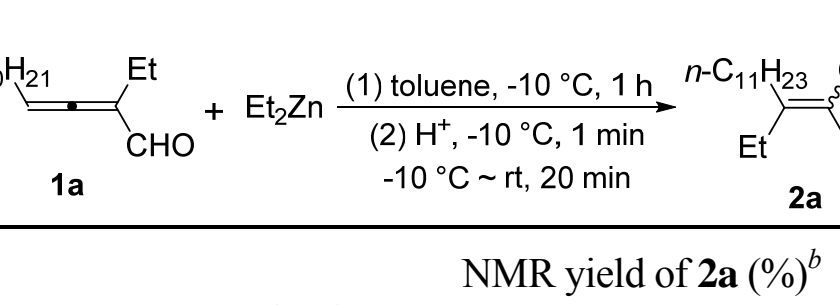 }} & \multirow{2}{*}{\multicolumn{2}{|c|}{$\frac{\mathrm{Na}}{\mathrm{NMR} \text { yield of } 3 \mathbf{a}(\%)^{b}}$}} \\
\hline & & & & & \\
\hline & proton source $\left(\mathrm{p} K_{\mathrm{a}}\right)$ & $(Z)-\mathbf{2 a}$ & $(E)-\mathbf{2 a}$ & $(Z)-3 a$ & $(E)-\mathbf{3 a}$ \\
\hline 1 & $\mathrm{CHF}_{2} \mathrm{COOH}(1.24)$ & $\sim 52$ & $\sim 21$ & 9.5 & 1.1 \\
\hline 2 & $\mathrm{CF}_{3} \mathrm{CH}_{2} \mathrm{COOH}(3.07)$ & 86 & n.d. & 1.7 & n.d. \\
\hline 3 & HCOOH (3.77) & 88 & n.d. & 1.4 & n.d. \\
\hline 4 & $\mathrm{CH}_{3} \mathrm{COOH}(4.76)$ & 97 & n.d. & n.d. & n.d. \\
\hline 5 & $\mathrm{CH}_{3} \mathrm{CH}_{2} \mathrm{COOH}(4.87)$ & 94 & n.d. & n.d. & n.d. \\
\hline
\end{tabular}

${ }^{a}$ Reagents and conditions: $1 \mathrm{a}(0.2 \mathrm{mmol}), \mathrm{Et}_{2} \mathrm{Zn}(0.48 \mathrm{mmol})$, toluene $(4 \mathrm{~mL})$, and proton source $(7$ mmol). ${ }^{b}$ Determined by NMR using dibromomethane as the internal standard.

Synthesis of 2-butyl-3-phenyl-4-(p-tolyl)but-2(E)-enal 2-(2,4-dinitrophenyl)hydrazone ((E)-4) ${ }^{3}$ (djx-4-019)

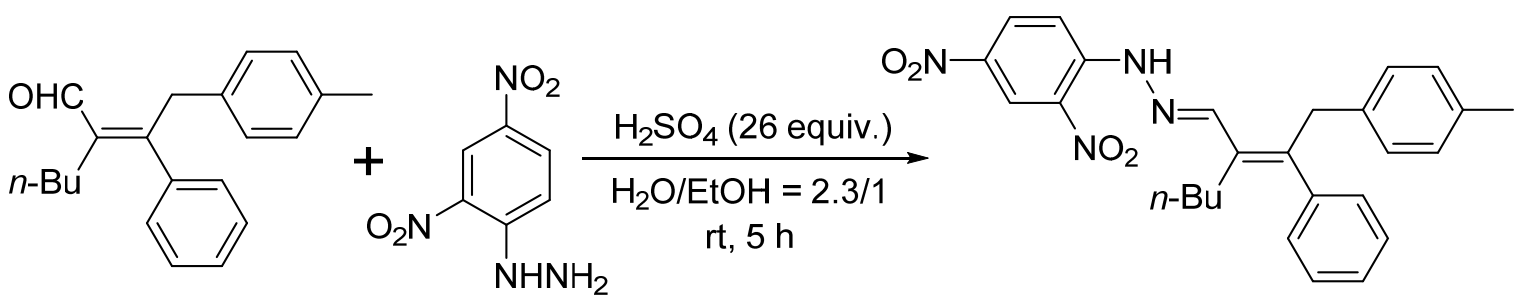
$(E)-\mathbf{2} \mathbf{w}$ $(E)-4,83 \%$

To a Schlenk tube were added 2,4-dinitrophenylhydrazine (0.0196 g, $0.1 \mathrm{mmol})$, $\mathrm{H}_{2} \mathrm{SO}_{4}\left(0.14 \mathrm{~mL}, d=1.84 \mathrm{~g} / \mathrm{cm}^{3}, 0.2576 \mathrm{~g}, 2.6 \mathrm{mmol}\right), \mathrm{EtOH}(0.8 \mathrm{~mL})$, and $\mathrm{H}_{2} \mathrm{O}(4.1$ $\mathrm{mL})$ sequentially under a nitrogen atmosphere. Then a solution of $(E)-2 \mathbf{w}(0.0292 \mathrm{~g}$, $0.1 \mathrm{mmol})$ in $\mathrm{EtOH}(1.0 \mathrm{~mL})$ was added via a syringe within 2 min with stirring. After being stirred for $5 \mathrm{~h}$, the reaction was complete as monitored by TLC. The resulting mixture was extracted with ethyl acetate $(10 \mathrm{~mL} \times 3)$. The combined organic layer was washed with brine $(10 \mathrm{~mL})$ and dried over anhydrous $\mathrm{Na}_{2} \mathrm{SO}_{4}$. After filtration and evaporation, the residue was purified by flash chromatography on silica gel (eluent: petroleum ether/ethyl acetate $=30 / 1)$ to afford $(E)-4(0.0391 \mathrm{~g}, 83 \%)$ as a solid: m.p. 140-142 ${ }^{\circ} \mathrm{C}$ (n-hexane/dichloromethane); ${ }^{1} \mathrm{H}$ NMR (300 MHz, $\left.\mathrm{CDCl}_{3}\right) \delta 11.22$ (s, $1 \mathrm{H}$, 
$\mathrm{NH}), 9.13(\mathrm{~d}, J=2.7 \mathrm{~Hz}, 1 \mathrm{H}, \mathrm{ArH}), 8.37(\mathrm{~s}, 1 \mathrm{H},=\mathrm{CH}), 8.32\left(\mathrm{dd}, J_{1}=9.6 \mathrm{~Hz}, J_{2}=2.7\right.$ Hz, 1H, ArH), 7.94 (d, J=9.6 Hz, 1H, ArH), 7.34-7.20 (m, 3H, ArH), 7.10-6.92 (m, 6H, ArH), $3.94\left(\mathrm{~s}, 2 \mathrm{H}, \mathrm{CH}_{2}\right), 2.39$ (t, $\left.J=7.8 \mathrm{~Hz}, 2 \mathrm{H}, \mathrm{CH}_{2}\right), 2.29$ (s, 3H, $\mathrm{CH}_{3}$ ), 1.56-1.41 (m, 2H, $\left.\mathrm{CH}_{2}\right), 1.33-1.15\left(\mathrm{~m}, 2 \mathrm{H}, \mathrm{CH}_{2}\right), 0.81\left(\mathrm{t}, J=7.2 \mathrm{~Hz}, 3 \mathrm{H}, \mathrm{CH}_{3}\right) ;{ }^{13} \mathrm{C}$ NMR (75 MHz, $\left.\mathrm{CDCl}_{3}\right) \delta 147.9,147.5,144.9,142.1,138.1,136.1,135.0,134.2$, $130.0,129.3,128.4,128.1,127.9,127.2,123.5,116.6,39.7,31.7,28.8,22.9,21.0$, 13.8; IR (KBr) v (cm $\left.{ }^{-1}\right) 3272,2956,2923,2854,1611,1514,1496,1452,1420,1325$, 1303, 1282, 1221, 1195, 1140, 1087; MS (70 ev, EI) $m / z(\%) 473\left(\mathrm{M}^{+}+1,19.64\right), 472$ $\left(\mathrm{M}^{+}, 11.12\right), 325$ (100); Elemental analysis calcd for $\mathrm{C}_{27} \mathrm{H}_{28} \mathrm{~N}_{4} \mathrm{O}_{4}(\%)$ : C, 68.63; $\mathrm{H}$, 5.97; N, 11.86; Found: C, 68.69; H, 6.07; N, 11.95.

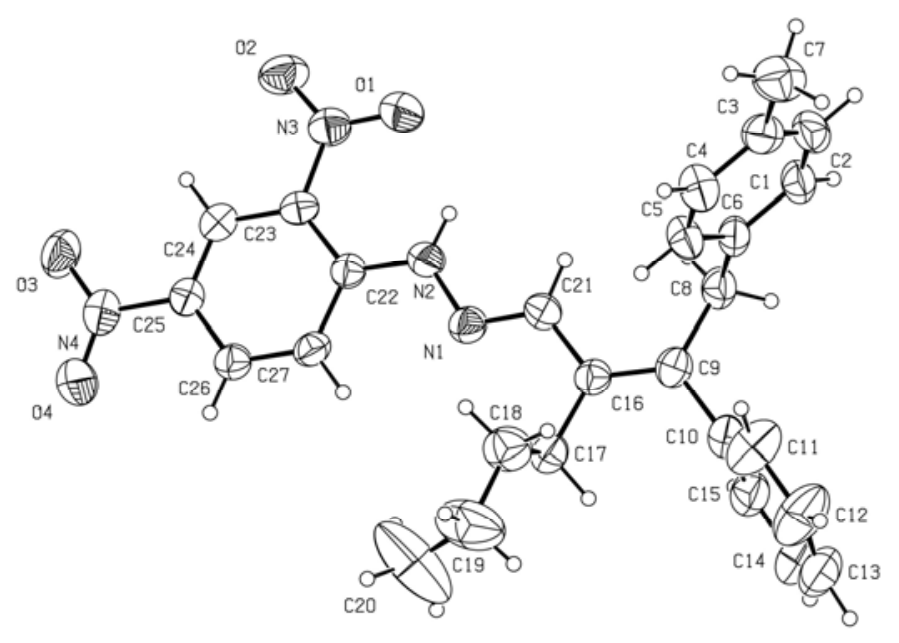

Figure S1. ORTEP Representation of $(E)-\mathbf{4}$.

Synthesis of 3-ethyl-2-propyl-4-(p-tolyl)but-1(Z),3(Z)-dienyl 1-acetate ((1Z,3Z)-5) and 3-ethyl-2-propyl-4-(p-tolyl)but-1(Z),3(E)-dienyl 1-acetate $\quad((1 Z, 3 E)-5)$ $(\mathrm{djx}-3-185)$

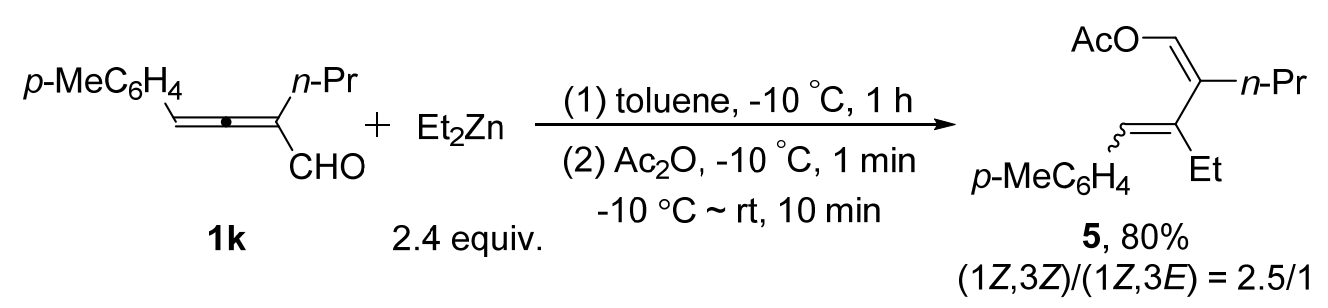

To a dry Schlenk flask $(50 \mathrm{~mL})$ were added $1 \mathbf{k}(0.0998 \mathrm{~g}, 0.5 \mathrm{mmol})$ and toluene $(10 \mathrm{~mL})$ under a nitrogen atmosphere. Then a solution of $\mathrm{Et}_{2} \mathrm{Zn}(0.6 \mathrm{~mL}, 2.0 \mathrm{M}$ in 
toluene, $1.2 \mathrm{mmol}$ ) was added via a syringe within $2 \mathrm{~min}$ with stirring at $-10^{\circ} \mathrm{C}$. After being stirred for $1 \mathrm{~h}$ at $-10{ }^{\circ} \mathrm{C}$, acetic anhydride $(1 \mathrm{~mL})$ was added via a syringe to the reaction mixture within $1 \mathrm{~min}$ at $-10{ }^{\circ} \mathrm{C}$, followed by warming up to room temperature with stirring. After $10 \mathrm{~min}$, ethyl acetate $(10 \mathrm{~mL})$ was added. The resulting mixture was washed sequentially with an aqueous solution of $\mathrm{HCl}(5 \%, 10 \mathrm{~mL})$, a saturated aqueous solution of $\mathrm{NaHCO}_{3}(10 \mathrm{~mL})$, and brine $(10 \mathrm{~mL})$. The combined aqueous layer was extracted with ethyl acetate $(10 \mathrm{~mL} \times 2)$. The combined organic layer was dried over anhydrous $\mathrm{Na}_{2} \mathrm{SO}_{4}$. After filtration and evaporation, the residue was added $35 \mu \mathrm{L}$ of dibromomethane for the ${ }^{1} \mathrm{H}$ NMR analysis $((1 Z, 3 Z) /(1 Z, 3 E)=2.5 / 1)$ and purified by flash chromatography on silica gel (eluent: petroleum ether/diethyl ether = $100 / 1)$ to afford $5(0.1090 \mathrm{~g}, 80 \%,(1 Z, 3 Z) /(1 Z, 3 E)=2.5 / 1)$ as an $E / Z$ mixture. Part of the two isomers were separated by preparative HPLC afforded pure (1Z,3Z)-5 and $(1 Z, 3 E)-5$.

(1Z,3Z)-5 (djx-3-185-1): liquid; ${ }^{1} \mathrm{H}$ NMR (300 MHz, $\left.\mathrm{CDCl}_{3}\right) \delta$ 7.25-7.20 (m, 2H, $\operatorname{ArH})$, 7.07-7.00 (m, 2H, ArH), 6.97 (t, $J=1.4 \mathrm{~Hz}, 1 \mathrm{H},=\mathrm{CH}), 6.29(\mathrm{~s}, 1 \mathrm{H},=\mathrm{CH})$, 2.33-2.24 (m, 5H, $\mathrm{CH}_{2}$ and $\left.\mathrm{CH}_{3}\right), 2.07-1.98\left(\mathrm{~m}, 2 \mathrm{H}, \mathrm{CH}_{2}\right), 1.94\left(\mathrm{~s}, 3 \mathrm{H}, \mathrm{CH}_{3}\right)$, 1.51-1.36 (m, 2H, $\left.\mathrm{CH}_{2}\right), 1.08\left(\mathrm{t}, J=7.5 \mathrm{~Hz}, 3 \mathrm{H}, \mathrm{CH}_{3}\right), 0.88\left(\mathrm{t}, J=7.2 \mathrm{~Hz}, 3 \mathrm{H}, \mathrm{CH}_{3}\right)$; ${ }^{13} \mathrm{C} \mathrm{NMR}\left(75 \mathrm{MHz}, \mathrm{CDCl}_{3}\right) \delta 168.2,139.0,136.1,135.0,130.9,128.7,127.9,125.7$, 125.6, 33.8, 30.6, 21.2, 21.1, 20.6, 14.1, 12.6; IR (neat) $v\left(\mathrm{~cm}^{-1}\right) 3019,2962,2930$, 2872, 1755, 1510, 1463, 1456, 1369, 1219, 1113, 1078; MS (70 ev, EI) m/z (\%) 272 $\left(\mathrm{M}^{+}, 4.34\right), 187$ (100); HRMS calcd for $\mathrm{C}_{18} \mathrm{H}_{24} \mathrm{O}_{2}\left[\mathrm{M}^{+}\right]$: 272.1776 , found: 272.1780.

(1Z,3E)-5 (djx-3-185-2): liquid; ${ }^{1} \mathrm{H}$ NMR $\left(300 \mathrm{MHz}, \mathrm{CDCl}_{3}\right) \delta$ 7.23-7.12 (m, 4H, $\operatorname{ArH}), 7.01(\mathrm{~s}, 1 \mathrm{H},=\mathrm{CH}), 6.32(\mathrm{~s}, 1 \mathrm{H},=\mathrm{CH}), 2.47-2.32\left(\mathrm{~m}, 5 \mathrm{H}, \mathrm{CH}_{2}\right.$ and $\left.\mathrm{CH}_{3}\right)$, 2.17-2.05 (m, 5H, $\mathrm{CH}_{2}$ and $\left.\mathrm{CH}_{3}\right), 1.51-1.37\left(\mathrm{~m}, 2 \mathrm{H}, \mathrm{CH}_{2}\right), 1.00(\mathrm{t}, J=7.5 \mathrm{~Hz}, 3 \mathrm{H}$, $\left.\mathrm{CH}_{3}\right), 0.92\left(\mathrm{t}, J=7.2 \mathrm{~Hz}, 3 \mathrm{H}, \mathrm{CH}_{3}\right) ;{ }^{13} \mathrm{C} \mathrm{NMR}\left(75 \mathrm{MHz}, \mathrm{CDCl}_{3}\right) \delta 168.4,139.1$, 136.1, 134.9, 130.6, 128.9, 128.7, 128.4, 127.7, 34.1, 22.6, 21.2, 21.1, 20.8, 13.7, 12.9; IR (neat) $v\left(\mathrm{~cm}^{-1}\right)$ 3020, 2961, 2929, 2872, 1755, 1510, 1463, 1371, 1219, 1111 , 1076, 1065; MS (70 ev, EI) $m / z(\%) 272\left(\mathrm{M}^{+}, 3.51\right), 187$ (100); HRMS calcd for $\mathrm{C}_{18} \mathrm{H}_{24} \mathrm{O}_{2}\left[\mathrm{M}^{+}\right]$: 272.1776, found: 272.1779 . 
Deuterium-labelling study (djx-3-184)

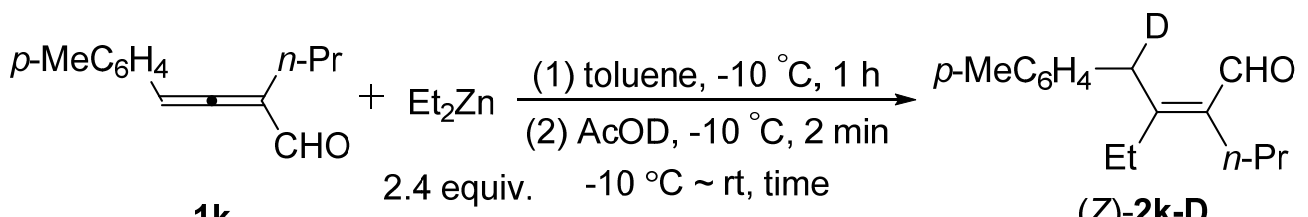

To a dry Schlenk flask $(100 \mathrm{~mL})$ were added $1 \mathbf{k}(0.2001 \mathrm{~g}, 1 \mathrm{mmol})$ and toluene $(20 \mathrm{~mL})$ under a nitrogen atmosphere. Then a solution of $\mathrm{Et}_{2} \mathrm{Zn}$ in toluene $(1.2 \mathrm{~mL}$, 2.0 M, $2.4 \mathrm{mmol}$ ) was added via a syringe within 4 min with stirring at $-10{ }^{\circ} \mathrm{C}$. After being stirred for $1 \mathrm{~h}$ at $-10{ }^{\circ} \mathrm{C}$, DOAc $(2 \mathrm{~mL})$ was added via a syringe to the reaction mixture within 2 min at $-10{ }^{\circ} \mathrm{C}$, followed by warming up to room temperature with stirring. Each of $2.2 \mathrm{~mL}$ of the resulting mixture was taken after $2 \mathrm{~min}, 4 \mathrm{~min}, 6 \mathrm{~min}$, $8 \mathrm{~min}, 10 \mathrm{~min}, 13 \mathrm{~min}, 17 \mathrm{~min}$, and $21 \mathrm{~min}$ respectively via syringes; After evaporation, the deuteration rates of these eight samples were determined by ${ }^{1} \mathrm{H}$ NMR analysis with the results being summarized in Table S2. The residue (21 min) was purified by flash chromatography on silica gel (eluent: petroleum ether (30-60 $\left.{ }^{\circ} \mathrm{C}\right) /$ diethyl ether $\left.=100 / 1\right)$ to afford $(Z)-2 \mathbf{k}-\mathbf{D}(0.0195 \mathrm{~g}, 92 \% \mathrm{D})$ as a liquid: ${ }^{1} \mathrm{H}$ NMR $\left(300 \mathrm{MHz}, \mathrm{CDCl}_{3}\right) \delta 10.18(\mathrm{~s}, 1 \mathrm{H}, \mathrm{CHO}), 7.11(\mathrm{t}, J=7.8 \mathrm{~Hz}, 2 \mathrm{H}, \mathrm{ArH}), 7.04(\mathrm{t}, J=$ 8.1 Hz, 2H, ArH), $3.88(\mathrm{~s}, 1 \mathrm{H}, \mathrm{CHD}), 2.37-2.17\left(\mathrm{~m}, 7 \mathrm{H}, \mathrm{CH}_{3}\right.$ and $\left.\mathrm{CH}_{2} \times 2\right), 1.47-1.32$ $\left(\mathrm{m}, 2 \mathrm{H}, \mathrm{CH}_{2}\right), 1.06\left(\mathrm{t}, J=7.5 \mathrm{~Hz}, 3 \mathrm{H}, \mathrm{CH}_{3}\right), 0.94\left(\mathrm{t}, J=7.2 \mathrm{~Hz}, 3 \mathrm{H}, \mathrm{CH}_{3}\right)$; the following signal is discernible for $(Z)-2 \mathbf{k}$ : $\delta 3.90\left(\mathrm{~s}, 2 \mathrm{H}, \mathrm{CH}_{2}\right) ;{ }^{13} \mathrm{C} \mathrm{NMR}(75 \mathrm{MHz}$, $\left.\mathrm{CDCl}_{3}\right) \delta 192.0,161.1,137.9,136.2,135.3,129.3,128.4,34.3\left(\mathrm{t}, J_{C-D}=19.7 \mathrm{~Hz}\right)$, 30.9, 27.4, 27.0, 22.8, 20.9, 14.3, 12.7; IR (neat) $v\left(\mathrm{~cm}^{-1}\right)$ 3021, 2962, 2932, 2871, $2763,1668,1612,1513,1463,1410,1377,1319,1299,1224,1151,1117,1067$; MS (70 ev, EI) $m / z(\%) 231\left(\mathrm{M}^{+}, 6.02\right), 230$ (16.68), 187 (100); HRMS calcd for $\mathrm{C}_{16} \mathrm{H}_{21} \mathrm{DO}\left[\mathrm{M}^{+}\right]: 231.1733$, found: 231.1730 .

Table S2 D-Incorporation vs. reaction time

\begin{tabular}{|c|c|c|c|c|c|c|c|c|}
\hline Time (min) & 2 & 4 & 6 & 8 & 10 & 13 & 17 & 21 \\
\hline D (\%) & 15 & 22 & 33 & 39 & 48 & 65 & 86 & 92 \\
\hline
\end{tabular}

\section{Synthesis of (Z)-2a from deprotonation of 3a with $\mathbf{E t}_{2} \mathbf{Z n}(\operatorname{djx}-5-042)$}




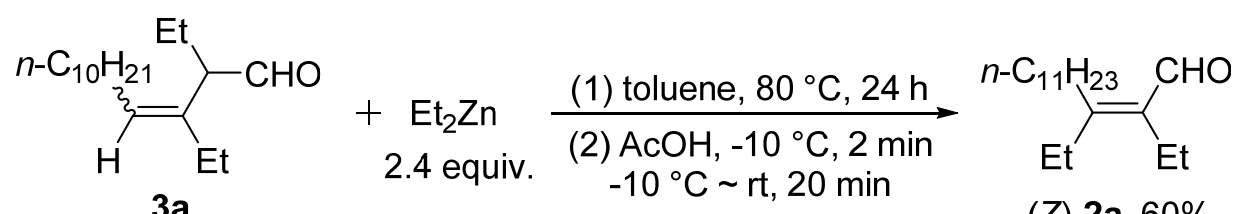

(Z)-2a, 60\%

To a dry Schlenk tube were added 3a $(0.0532 \mathrm{~g}, 0.2 \mathrm{mmol})$ and toluene $(4 \mathrm{~mL})$ under a nitrogen atmosphere. Then a solution of $\mathrm{Et}_{2} \mathrm{Zn}$ in toluene $(0.24 \mathrm{~mL}, 2.0 \mathrm{M}$, $0.48 \mathrm{mmol}$ ) was added within 2 min with stirring at room temperature. After being stirred in an oil bath preheated at $80{ }^{\circ} \mathrm{C}$ for $24 \mathrm{~h}$, $\mathrm{AcOH}(0.4 \mathrm{~mL})$ was added to the reaction mixture within 2 min at $-10{ }^{\circ} \mathrm{C}$, followed by warming up to room temperature with stirring. After $20 \mathrm{~min}$, ethyl acetate $(10 \mathrm{~mL})$ was added. The resulting mixture was washed sequentially with an aqueous solution of $\mathrm{HCl}(5 \%, 10 \mathrm{~mL})$, a saturated aqueous solution of $\mathrm{NaHCO}_{3}(10 \mathrm{~mL})$, and brine $(10 \mathrm{~mL})$. The combined aqueous layer was extracted with ethyl acetate $(10 \mathrm{~mL} \times 2)$. The combined organic layer was dried over anhydrous $\mathrm{Na}_{2} \mathrm{SO}_{4}$. After filtration and evaporation, the residue was purified by flash chromatography on silica gel (eluent: petroleum ether (30-60 $\left.{ }^{\circ} \mathrm{C}\right) /$ diethyl ether $\left.=100 / 1\right)$ to afford $(Z)-2 \mathrm{a}(0.0317 \mathrm{~g}, 60 \%)$ as a liquid: ${ }^{1} \mathrm{H}$ NMR $(300$ $\left.\mathrm{MHz}, \mathrm{CDCl}_{3}\right) \delta 10.06(\mathrm{~s}, 1 \mathrm{H}, \mathrm{CHO}), 2.52\left(\mathrm{t}, J=8.0 \mathrm{~Hz}, 2 \mathrm{H}, \mathrm{CH}_{2}\right), 2.34-2.18(\mathrm{~m}, 4 \mathrm{H}$, $\left.\mathrm{CH}_{2} \times 2\right), 1.56-1.41\left(\mathrm{~m}, 2 \mathrm{H}, \mathrm{CH}_{2}\right), 1.39-1.19\left(\mathrm{~m}, 16 \mathrm{H}, \mathrm{CH}_{2} \times 8\right), 1.10(\mathrm{t}, J=7.7 \mathrm{~Hz}$, $\left.3 \mathrm{H}, \mathrm{CH}_{3}\right), 0.94\left(\mathrm{t}, J=7.4 \mathrm{~Hz}, 3 \mathrm{H}, \mathrm{CH}_{3}\right), 0.88\left(\mathrm{t}, J=6.8 \mathrm{~Hz}, 3 \mathrm{H}, \mathrm{CH}_{3}\right) ;{ }^{13} \mathrm{C}$ NMR $(75$ $\left.\mathrm{MHz}, \mathrm{CDCl}_{3}\right) \delta 191.5,164.1,137.9,31.9,30.9,30.2,29.7,29.6,29.5,29.4,29.3,27.5$, $22.7,18.4,14.1,12.8$.

Synthesis of 4-ethyl-3-propyltrideca-3(Z)-en-1-yne ((Z)-8) ${ }^{4}(\operatorname{djx}-3-119)$

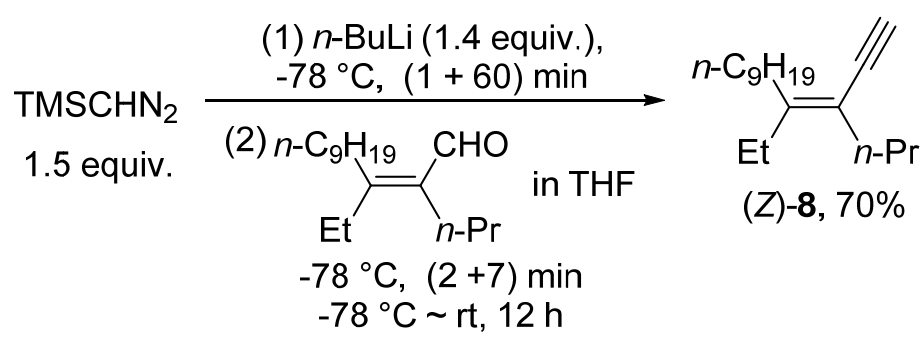

To a dry Schlenk tube was added a solution of (trimethylsilyl)diazomethane in hexane $(0.375 \mathrm{~mL}, 2.0 \mathrm{M}, 0.75 \mathrm{mmol})$ at $-78{ }^{\circ} \mathrm{C}$ under a nitrogen atmosphere. Then a solution of $n$-BuLi $(0.28 \mathrm{~mL}, 2.5 \mathrm{M}$ in hexane, $0.7 \mathrm{mmol})$ was added via a syringe 
within 1 min with stirring at $-78{ }^{\circ} \mathrm{C}$. After $1 \mathrm{~h}$, a solution of (Z)-3-ethyl-2-propyldodec -2-enal $(0.1260 \mathrm{~g}, 0.5 \mathrm{mmol})$ in THF $(3 \mathrm{~mL})$ was added via a syringe to the reaction mixture within 2 min at $-78{ }^{\circ} \mathrm{C}$. The resulting mixture was allowed to stir at $-78{ }^{\circ} \mathrm{C}$ for $7 \mathrm{~min}$, followed by warming up to room temperature. After $12 \mathrm{~h}$ with stirring, the reaction was complete as monitored by TLC. The resulting mixture was quenched with a saturated aqueous solution of $\mathrm{NH}_{4} \mathrm{Cl}(10 \mathrm{~mL})$ and extracted with diethyl ether $(10 \mathrm{~mL} \times 3)$. The combined organic layer was dried over anhydrous $\mathrm{Na}_{2} \mathrm{SO}_{4}$. After filtration and evaporation, the residue was purified by flash chromatography on silica gel (eluent: petroleum ether $\left.\left(30-60{ }^{\circ} \mathrm{C}\right)\right)$ to afford $(Z)-8(0.0868 \mathrm{~g}, 70 \%)$ as a liquid: ${ }^{1} \mathrm{H}$ NMR $\left(300 \mathrm{MHz}, \mathrm{CDCl}_{3}\right) \delta 2.98(\mathrm{~s}, 1 \mathrm{H}, \equiv \mathrm{CH}), 2.33\left(\mathrm{t}, J=7.7 \mathrm{~Hz}, 2 \mathrm{H}, \mathrm{CH}_{2}\right)$, 2.20-2.02 (m, 4H, $\left.\mathrm{CH}_{2} \times 2\right), 1.64-1.15\left(\mathrm{~m}, 16 \mathrm{H}, \mathrm{CH}_{2} \times 8\right), 1.09-0.77\left(\mathrm{~m}, 9 \mathrm{H}, \mathrm{CH}_{3} \times 3\right)$; ${ }^{13} \mathrm{C}$ NMR $\left(75 \mathrm{MHz}, \mathrm{CDCl}_{3}\right) \delta 151.8,116.3,84.7,78.7,34.6,33.5,31.9,29.64,29.59$, 29.5, 29.4, 28.3, 24.2, 22.7, 22.0, 14.1, 13.7, 13.0; IR (neat) $v\left(\mathrm{~cm}^{-1}\right) 3312,2960$, 2925, 2872, 2855, 2088, 1463, 1377, 1261, 1176, 1132, 1064; MS (70 ev, EI) m/z (\%) $248\left(\mathrm{M}^{+}, 11.09\right), 93$ (100); HRMS calcd for $\mathrm{C}_{18} \mathrm{H}_{32}\left[\mathrm{M}^{+}\right]$: 248.2504, found: 248.2509.

Synthesis of 5-ethyl-4-propyltetradec-4(Z)-en-1-yn-3-ol ((Z)-9) ${ }^{5}(\operatorname{djx}-3-153)$

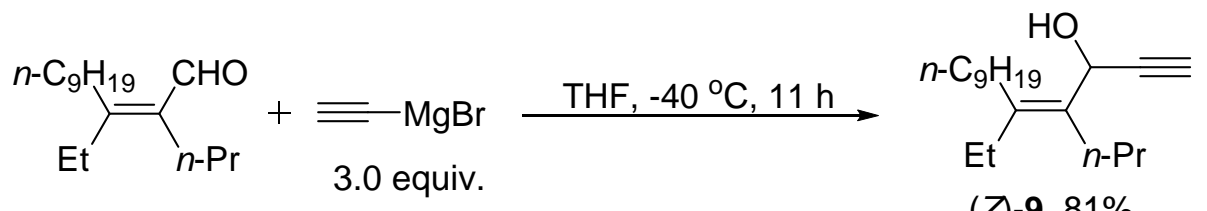

(Z)-9, 81\%

To a dry Schlenk tube was added (Z)-3-ethyl-2-propyldodec-2-enal $(0.1261 \mathrm{~g}, 0.5$ mmol) and THF (2 $\mathrm{mL})$ under a nitrogen atmosphere. Then a solution of ethynylmagnesium bromide in THF $(3.0 \mathrm{~mL}, 0.5 \mathrm{M}, 1.5 \mathrm{mmol})$ was added via a syringe within $2 \mathrm{~min}$ with stirring at $-40{ }^{\circ} \mathrm{C}$. After being stirred for $11 \mathrm{~h}$ at $-40{ }^{\circ} \mathrm{C}$, the reaction was complete as monitored by TLC. The resulting mixture was quenched with a saturated aqueous solution of $\mathrm{NH}_{4} \mathrm{Cl}(5 \mathrm{~mL})$ at $-40{ }^{\circ} \mathrm{C}$, followed by warming up to room temperature. The resulting mixture was extracted with diethyl ether $(10 \mathrm{~mL} \times$ 3). The combined organic layer was dried over anhydrous $\mathrm{Na}_{2} \mathrm{SO}_{4}$. After filtration and evaporation, the residue was purified by flash chromatography on silica gel (eluent: 
petroleum ether/ethyl acetate $=50 / 1)$ to afford $(Z)-9(0.1126 \mathrm{~g}, 81 \%)$ as a liquid: ${ }^{1} \mathrm{H}$ NMR (300 MHz, $\left.\mathrm{CDCl}_{3}\right) \delta 5.29$ (bs, 1H, CH), $2.45(\mathrm{~d}, J=2.1 \mathrm{~Hz}, 1 \mathrm{H}, \equiv \mathrm{CH})$, 2.25-1.91 (m, 7H, $\mathrm{CH}_{2} \times 3$ and $\left.\mathrm{OH}\right), 1.68-1.19\left(\mathrm{~m}, 16 \mathrm{H}, \mathrm{CH}_{2} \times 8\right), 1.05-0.82(\mathrm{~m}, 9 \mathrm{H}$, $\left.\mathrm{CH}_{3} \times 3\right) ;{ }^{13} \mathrm{C} \mathrm{NMR}\left(75 \mathrm{MHz}, \mathrm{CDCl}_{3}\right) \delta 141.3,131.5,84.4,72.8,61.9,31.8,31.1$, $30.6,29.9,29.54,29.52,29.46,29.2,25.3,24.2,22.6,14.6,14.0,13.1$; IR (neat) $v$ $\left(\mathrm{cm}^{-1}\right)$ 3444, 3311, 2959, 2927, 2871, 2855, 2111, 1466, 1376, 1304, 1247, 1067, 1006; MS (70 ev, EI) m/z (\%) $278\left(\mathrm{M}^{+}, 16.10\right), 151$ (100); HRMS calcd for $\mathrm{C}_{19} \mathrm{H}_{34} \mathrm{O}$ $\left[\mathrm{M}^{+}\right]: 278.2610$, found: 278.2613 .

Synthesis of 4-ethyl-3-propyltridec-3(Z)-en-2-ol ((Z)-10) ${ }^{6}$ (djx-3-154)

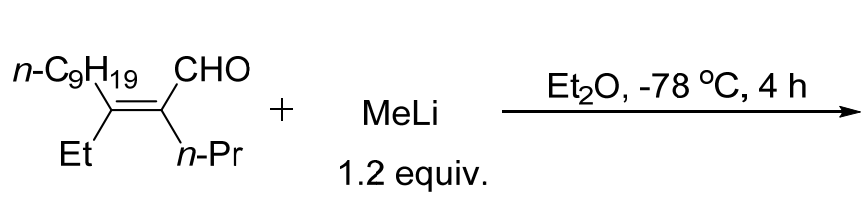

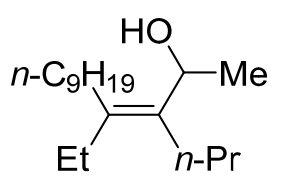

(Z)-10, 85\%

To a dry Schlenk tube were added (Z)-3-ethyl-2-propyldodec-2-enal (0.1261 g, 0.5 mmol) and $\mathrm{Et}_{2} \mathrm{O}(3 \mathrm{~mL})$ under a nitrogen atmosphere. Then a solution of methyllithium in $\mathrm{Et}_{2} \mathrm{O}(0.2 \mathrm{~mL}, 3.0 \mathrm{M}, 0.6 \mathrm{mmol})$ was added via a syringe within 1 min with stirring at $-78{ }^{\circ} \mathrm{C}$. After being stirred for $4 \mathrm{~h}$ at $-78{ }^{\circ} \mathrm{C}$, the reaction was complete as monitored by TLC. The resulting mixture was quenched with a saturated aqueous solution of $\mathrm{NH}_{4} \mathrm{Cl}(3 \mathrm{~mL})$ at $-78{ }^{\circ} \mathrm{C}$, followed by warming up to room temperature. The resulting mixture was extracted with diethyl ether $(10 \mathrm{~mL} \times 3)$. The combined organic layer was dried over anhydrous $\mathrm{Na}_{2} \mathrm{SO}_{4}$. After filtration and evaporation, the residue was purified by flash chromatography on silica gel (eluent: petroleum ether/ethyl acetate $=50 / 1)$ to afford $(Z)-10(0.1138 \mathrm{~g}, 85 \%)$ as a liquid: ${ }^{1} \mathrm{H}$ NMR (300 MHz, $\left.\mathrm{CDCl}_{3}\right) \delta 4.79(\mathrm{q}, J=6.5 \mathrm{~Hz}, 1 \mathrm{H}, \mathrm{CH}), 2.17-1.89\left(\mathrm{~m}, 6 \mathrm{H}, \mathrm{CH}_{2} \times 3\right)$, 1.56-1.16 (m, 20H, $\mathrm{CH}_{2} \times 8, \mathrm{CH}_{3}$, and $\left.\mathrm{OH}\right), 1.04-0.82\left(\mathrm{~m}, 9 \mathrm{H}, \mathrm{CH}_{3} \times 3\right) ;{ }^{13} \mathrm{C} \mathrm{NMR}$ $\left(75 \mathrm{MHz}, \mathrm{CDCl}_{3}\right) \delta 138.2,135.6,67.2,31.8,30.4,30.0,29.9,29.6,29.5,29.3,28.9$, 25.1, 24.9, 22.6, 21.7, 14.8, 14.0, 13.2; IR (neat) $v\left(\mathrm{~cm}^{-1}\right) 3396,2959,2926,2871$, 2855, 1466, 1375, 1254, 1153, 1092, 1051; MS (70 ev, EI) m/z (\%) $268\left(\mathrm{M}^{+}, 4.90\right), 43$ (100); HRMS calcd for $\mathrm{C}_{18} \mathrm{H}_{36} \mathrm{O}\left[\mathrm{M}^{+}\right]$: 268.2766, found: 268.2770 . 
Synthesis of 4-ethyl-3-propyltrideca-1,3(Z)-diene ((Z)-11) ${ }^{7}(\operatorname{djx}-3-124)$

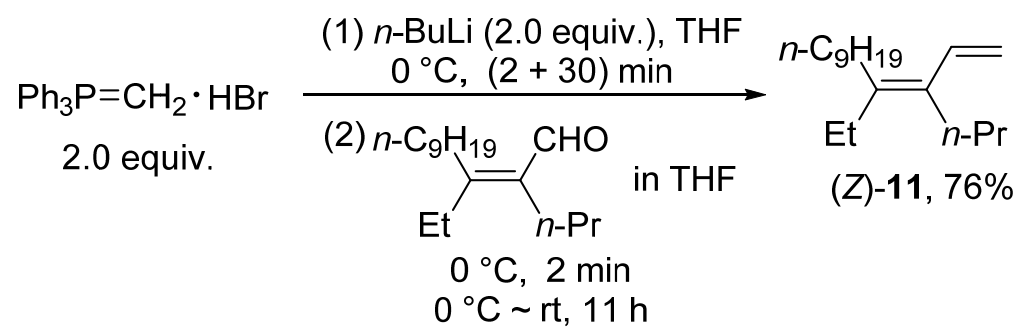

To a dry Schlenk tube were added methyltriphenylphosphonium bromide $(0.3605 \mathrm{~g}$, $1.0 \mathrm{mmol})$ and THF $(2 \mathrm{~mL})$ under a nitrogen atmosphere. Then a solution of $n$-BuLi in hexane $(0.4 \mathrm{~mL}, 2.5 \mathrm{M}, 1.0 \mathrm{mmol})$ was added via a syringe within 2 min with stirring at $0{ }^{\circ} \mathrm{C}$. After $30 \mathrm{~min}$, a solution of (Z)-3-ethyl-2-propyldodec-2-enal (0.1257 $\mathrm{g}, 0.5 \mathrm{mmol})$ in THF $(3 \mathrm{~mL})$ was added via a syringe to the reaction mixture within 2 min at $0{ }^{\circ} \mathrm{C}$, followed by warming up to room temperature with stirring. After being stirred for $11 \mathrm{~h}$, the reaction was complete as monitored by TLC. The resulting mixture was quenched with brine $(10 \mathrm{~mL})$ and extracted with diethyl ether $(10 \mathrm{~mL} \times$ 3). The combined organic layer was dried over anhydrous $\mathrm{Na}_{2} \mathrm{SO}_{4}$. After filtration and evaporation, the residue was purified by flash chromatography on silica gel (eluent: petroleum ether $\left.\left(30-60{ }^{\circ} \mathrm{C}\right)\right)$ to afford $(Z)-11(0.0944 \mathrm{~g}, 76 \%)$ as a liquid: ${ }^{1} \mathrm{H}$ NMR $\left(300 \mathrm{MHz}, \mathrm{CDCl}_{3}\right) \delta 6.68\left(\mathrm{dd}, J_{l}=11.1 \mathrm{~Hz}, J_{2}=8.7 \mathrm{~Hz}, 1 \mathrm{H},=\mathrm{CH}\right), 5.11\left(\mathrm{dd}, J_{l}=8.7\right.$ $\mathrm{Hz}, J_{2}=1.5 \mathrm{~Hz}, 1 \mathrm{H}$, one porton of $\left.=\mathrm{CH}_{2}\right), 4.96\left(\mathrm{dd}, J_{1}=11.1 \mathrm{~Hz}, J_{2}=1.5 \mathrm{~Hz}, 1 \mathrm{H}\right.$, one porton of $\left.=\mathrm{CH}_{2}\right), 2.25-2.06\left(\mathrm{~m}, 6 \mathrm{H}, \mathrm{CH}_{2} \times 3\right), 1.49-1.19\left(\mathrm{~m}, 16 \mathrm{H}, \mathrm{CH}_{2} \times 8\right)$, 1.04-0.76 (m, 9H, $\left.\mathrm{CH}_{3} \times 3\right) ;{ }^{13} \mathrm{C} \mathrm{NMR}\left(75 \mathrm{MHz}, \mathrm{CDCl}_{3}\right) \delta 142.2,134.8,131.2,110.9$, $31.9,31.4,30.0,29.7,29.64,29.59,29.5,29.4,26.2,22.8,22.7,14.4,14.1,13.3$; IR (neat) $v\left(\mathrm{~cm}^{-1}\right) 3087,3018,2958,2926,2869,2855,1624,1593,1464,1412,1376$, 1347, 1302, 1162, 1091, 1066; MS (70 ev, EI) m/z (\%) $251\left(\mathrm{M}^{+}+1,6.70\right), 250\left(\mathrm{M}^{+}\right.$, 32.46), 95 (100); HRMS calcd for $\mathrm{C}_{18} \mathrm{H}_{34}\left[\mathrm{M}^{+}\right]$: 250.2661, found: 250.2656 .

Synthesis of 3-ethyl-2-propyldodec-2(Z)-en-1-ol ((Z)-12) ${ }^{8}\left(\operatorname{djx}^{\mathrm{x}-4-073)}\right.$ 


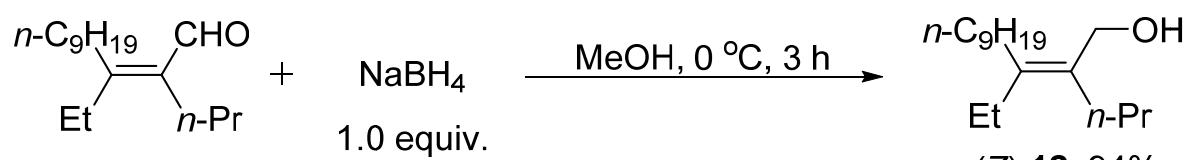

To a dry Schlenk tube was added (Z)-3-ethyl-2-propyldodec-2-enal (0.1260 g, 0.5 mmol) and $\mathrm{MeOH}(4 \mathrm{~mL})$ under a nitrogen atmosphere. Then $\mathrm{NaBH}_{4}(0.0195 \mathrm{~g}, 0.5$ mmol) was added with stirring at $0{ }^{\circ} \mathrm{C}$. After being stirred for $3 \mathrm{~h}$ at $0{ }^{\circ} \mathrm{C}$, the reaction was complete as monitored by TLC. The resulting mixture was quenched with a saturated aqueous solution of $\mathrm{NH}_{4} \mathrm{Cl}(4 \mathrm{~mL})$ and extracted with diethyl ether $(5 \mathrm{~mL} \times$ 3). The combined organic layer was dried over anhydrous $\mathrm{Na}_{2} \mathrm{SO}_{4}$. After filtration and evaporation, the residue was purified by flash chromatography on silica gel (eluent: petroleum ether/ethyl acetate $=20 / 1)$ to afford $(Z)-\mathbf{1 2}(0.1194 \mathrm{~g}, 94 \%)$ as a liquid: ${ }^{1} \mathrm{H}$ NMR (300 MHz, $\left.\mathrm{CDCl}_{3}\right) \delta 4.09\left(\mathrm{~s}, 2 \mathrm{H}, \mathrm{CH}_{2}\right), 2.20-1.97\left(\mathrm{~m}, 6 \mathrm{H}, \mathrm{CH}_{2} \times 3\right), 1.57-1.16$ $\left(\mathrm{m}, 17 \mathrm{H}, \mathrm{CH}_{2} \times 8\right.$ and $\left.\mathrm{OH}\right), 1.05-0.81\left(\mathrm{~m}, 9 \mathrm{H}, \mathrm{CH}_{3} \times 3\right) ;{ }^{13} \mathrm{C} \mathrm{NMR}\left(75 \mathrm{MHz}, \mathrm{CDCl}_{3}\right)$ $\delta 140.6,132.3,61.7,32.1,31.8,31.2,29.9,29.6,29.3,24.8,22.6,22.4,14.2,14.0$, 13.4; IR (neat) $v\left(\mathrm{~cm}^{-1}\right) 3350,2959,2926,2871,2855,1465,1377,1261,1142,1067$, 1008; MS (70 ev, EI) m/z (\%) $254\left(\mathrm{M}^{+}, 11.29\right), 127$ (100); HRMS calcd for $\mathrm{C}_{17} \mathrm{H}_{34} \mathrm{O}$ $\left[\mathrm{M}^{+}\right]: 254.2610$, found: 254.2610 .

Synthesis of 3-ethyl-2-propyldodec-2(Z)-enoic acid ((Z)-13) ${ }^{9}(\operatorname{djx}-4-077)$

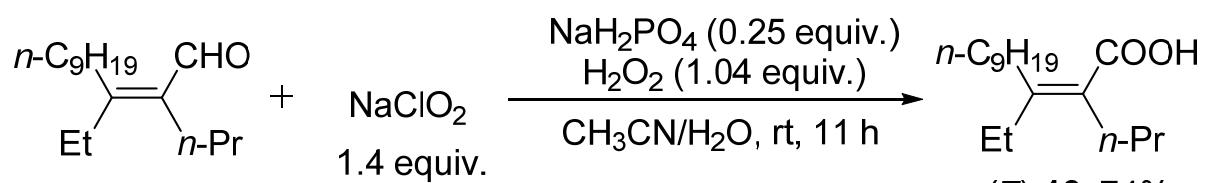

(Z)-13, 74\%

To a Schlenk tube was added (Z)-3-ethyl-2-propyldodec-2-enal $(0.1260 \mathrm{~g}, 0.5$ mmol), $\mathrm{CH}_{3} \mathrm{CN}(2 \mathrm{~mL})$, a solution of $\mathrm{NaH}_{2} \mathrm{PO}_{4}(0.0151 \mathrm{~g}, 0.125 \mathrm{mmol})$ in $\mathrm{H}_{2} \mathrm{O}(0.2$ $\mathrm{mL})$ and a aqueous solution of $\mathrm{H}_{2} \mathrm{O}_{2}\left(52 \mu \mathrm{L}, d=1.13 \mathrm{~g} / \mathrm{cm}^{3}, 0.0588 \mathrm{~g}, 30 \mathrm{wt} . \%, 0.52\right.$ mmol) sequentially. Then a solution of $\mathrm{NaClO}_{2}(0.0788 \mathrm{~g}, 0.7 \mathrm{mmol}, 80 \%)$ in $\mathrm{H}_{2} \mathrm{O}(2$ $\mathrm{mL}$ ) was added dropwise within $10 \mathrm{~min}$ with stirring. After being stirred for $11 \mathrm{~h}$, the reaction was complete as monitored by TLC. The resulting mixture was quenched with water $(50 \mathrm{~mL})$ and the $\mathrm{pH}$ value was adjusted to 10 with an aqueous solution of $\mathrm{NaOH}(1 \mathrm{M})$. The resulting mixture was washed with diethyl ether $(2 \mathrm{~mL} \times 2)$. Then 
the $\mathrm{pH}$ value was adjusted to 3 with an aqueous solution of $\mathrm{HCl}(5 \%)$. The aqueous layer was extracted with ethyl acetate $(10 \mathrm{~mL} \times 3)$. The combined organic layer was dried over anhydrous $\mathrm{Na}_{2} \mathrm{SO}_{4}$. After filtration and evaporation, the residue was purified by flash chromatography on silica gel (eluent: petroleum ether/ethyl acetate = 20/1) to afford (Z)-13 (0.0991 g, 74\%) as a liquid: ${ }^{1} \mathrm{H}$ NMR $\left(300 \mathrm{MHz}, \mathrm{CDCl}_{3}\right) \delta$ 11.93 (brs, 1H, COOH), 2.37 (t, $J=7.7 \mathrm{~Hz}, 2 \mathrm{H}, \mathrm{CH}_{2}$ ), 2.29 (t, $J=8.0 \mathrm{~Hz}, 2 \mathrm{H}, \mathrm{CH}_{2}$ ), $2.16\left(\mathrm{q}, J=7.5 \mathrm{~Hz}, 2 \mathrm{H}, \mathrm{CH}_{2}\right), 1.60-1.14\left(\mathrm{~m}, 16 \mathrm{H}, \mathrm{CH}_{2} \times 8\right), 1.04$ (t, $J=7.5 \mathrm{~Hz}, 3 \mathrm{H}$, $\left.\mathrm{CH}_{3}\right), 0.98-0.78\left(\mathrm{~m}, 6 \mathrm{H}, \mathrm{CH}_{3} \times 2\right) ;{ }^{13} \mathrm{C} \mathrm{NMR}\left(75 \mathrm{MHz}, \mathrm{CDCl}_{3}\right) \delta 175.9,155.0,126.9$, $33.8,31.9,31.3,29.9,29.6,29.44,29.35,29.1,26.6,22.73,22.69,14.1,13.9,12.8$; IR (neat) $v\left(\mathrm{~cm}^{-1}\right)$ 2958, 2926, 2871, 2855, 1687, 1682, 1463, 1378, 1289, 1254, 1229, 1075; MS (70 ev, EI) $m / z(\%) 268\left(\mathrm{M}^{+}, 75.74\right), 239$ (100); HRMS calcd for $\mathrm{C}_{17} \mathrm{H}_{32} \mathrm{O}_{2}$ $\left[\mathrm{M}^{+}\right]:$268.2402, found: 268.2400 . 


\section{References:}

(1) Yin, S.; Ma, S.; Ning, J.; Tao, F. Chin. J. Chem. 2002, 20, 707-710.

(2) Ma, S.; Liu, J.; Li, S.; Chen, B.; Cheng, J.; Kuang, J.; Liu, Y.; Wan, B.; Wang, Y.; Ye, J.; Yu, Q.; Yuan, W.; Yu, S. Adv. Synth. Catal. 2011, 353, 1005-1017.

(3) Crystal data for compound (E)-4: $\mathrm{C}_{27} \mathrm{H}_{28} \mathrm{~N}_{4} \mathrm{O}_{4} ; M W=472.53$; orthorhombic, space group Pbca; final $R$ indices $[I>2 \sigma(I)], R I=0.0952, w R 2=0.2182, R$ indices (all data) $R 1=0.1528, w R 2=0.2569 ; a=8.8093(14) \AA, b=18.443(2) \AA$, $c=31.202(5) \AA ; \alpha=90.00^{\circ}, \beta=90.00^{\circ}, \gamma=90.00^{\circ} ; V=5069.4(13) \AA^{3} ; T=293(2)$ $\mathrm{K} ; Z=8$; reflections collected/unique 18440/4611 $\left(R_{\text {int }}=0.0672\right)$; number of observations $[>2 \sigma(\mathrm{I})] 2565$, parameters: 318 . Supplementary crystallographic data have been deposited at the Cambridge Crystallographic Data Centre, CCDC 1431429.

(4) Sibilska, K.; Sicinski, R. R.; Ochalek, J. T.; Plum, L. A.; DeLuca, H. F. J. Med. Chem. 2014, 57, 8319-8331.

(5) Spencer, W. T.; Frontier, A. J. J. Org. Chem. 2012, 77, 7730-7736.

(6) Mao, G.; Wang, C.; Chen, J.; Muramatsub, A.; Xia, Z. Tetrahedron Lett. 2005, 46, $5369-5372$.

(7) Nani, R. R.; Reisman, S. E. J. Am. Chem. Soc. 2013, 135, 7304-7311.

(8) Schomaker, J. M.; Bhattacharjee, S.; Yan, J.; Borhan, B. J. Am. Chem. Soc. 2007, 129, 1996-2003.

(9) Dalcanale, E. J. Org. Chem. 1986, 51, 567-569. 

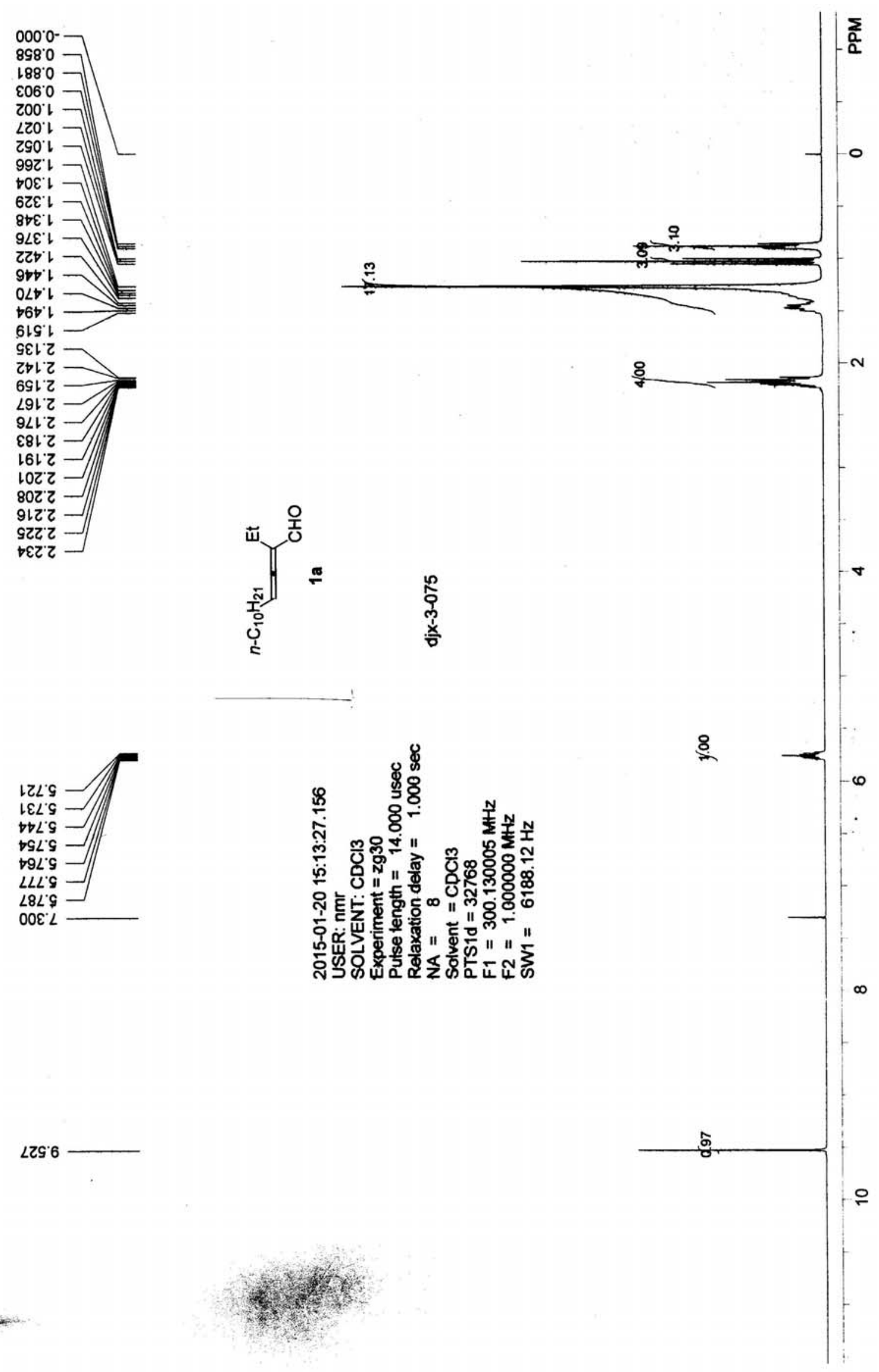

LZS' 6 

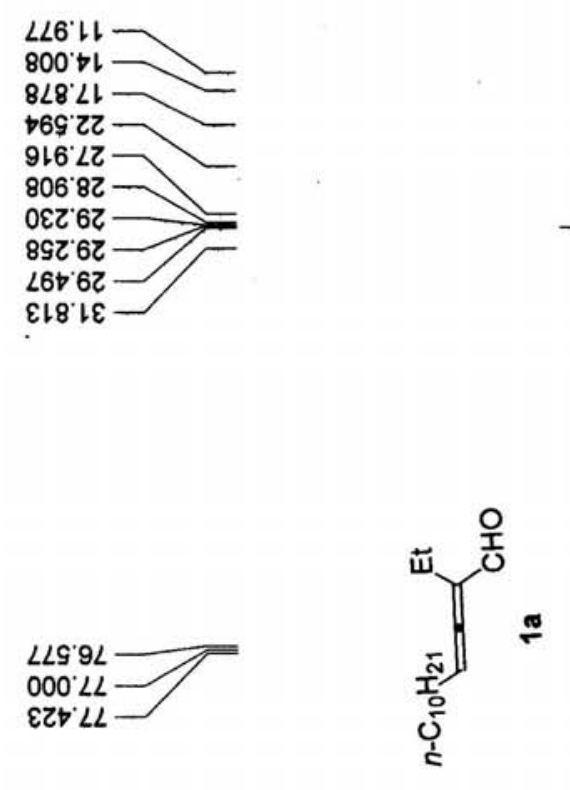

110266

乙Lยะเレ
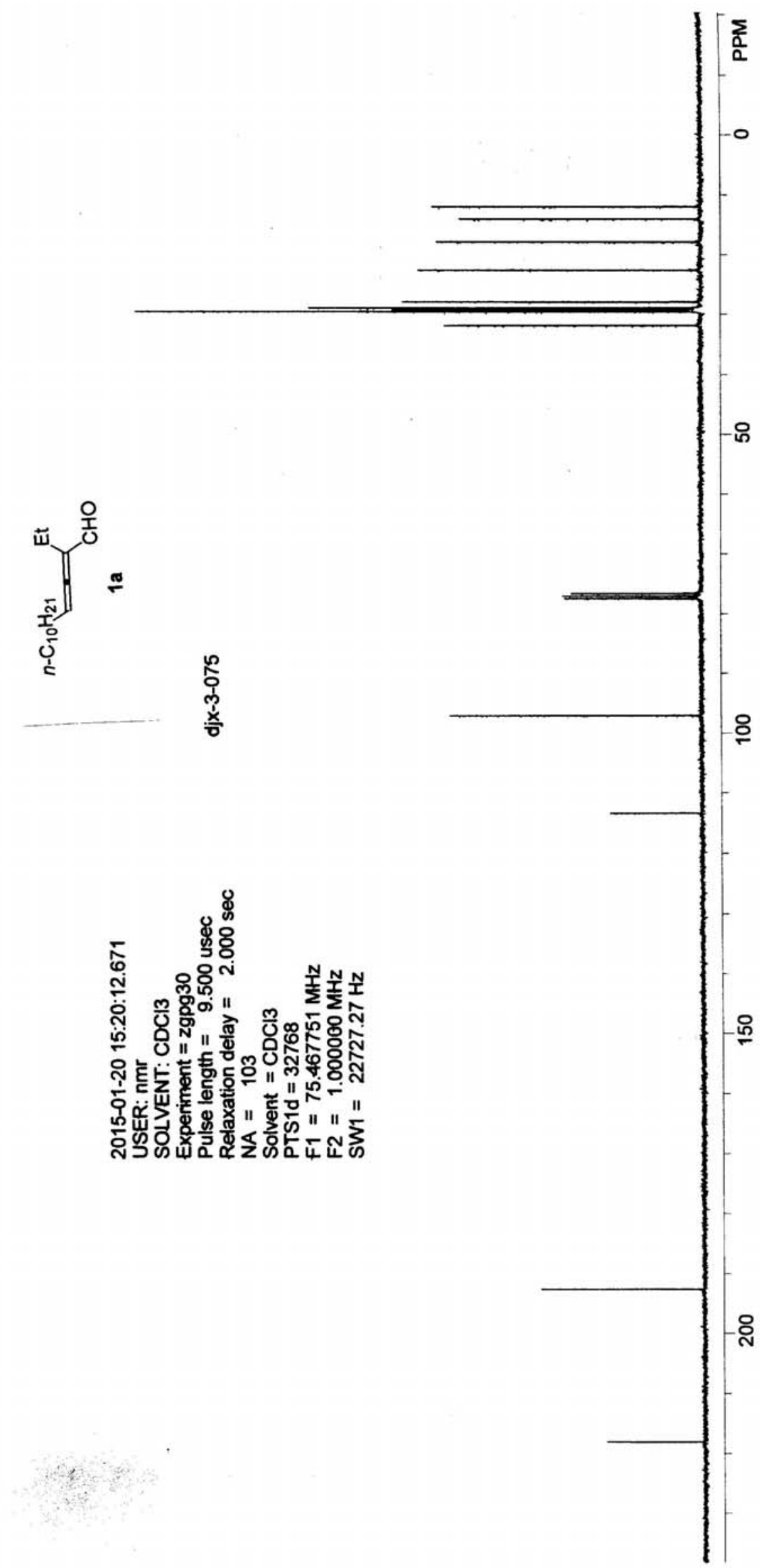

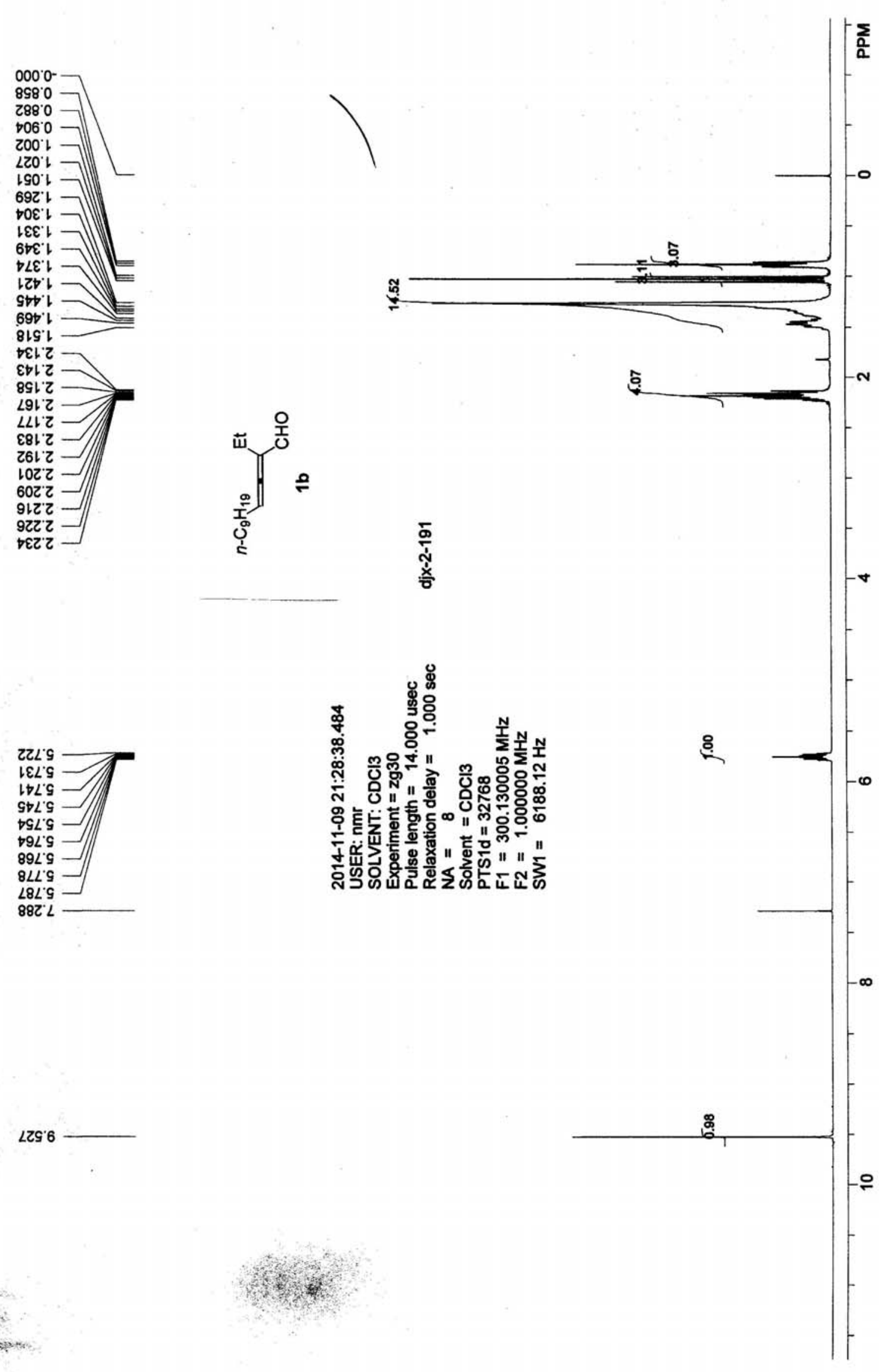

LZS' 6

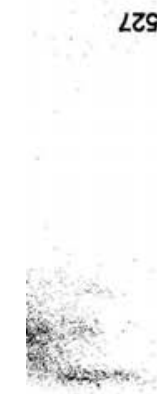


S00'Z

$\angle 20^{\circ}$

$\angle 68^{\circ} \mathrm{LL}$

\&09' 27

$\checkmark \varepsilon 6 . \angle Z$

$\angle 26.82$

เZZ 62

$9 L 262$

69t 62

乌6L'เE

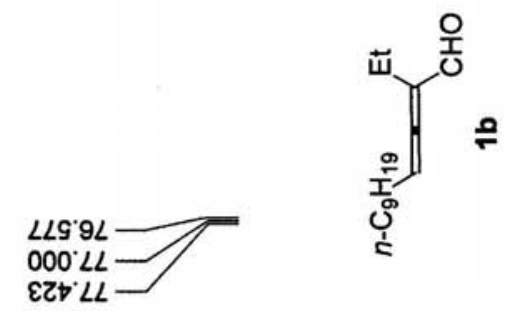

$\angle D O \angle 6$

00tEนL

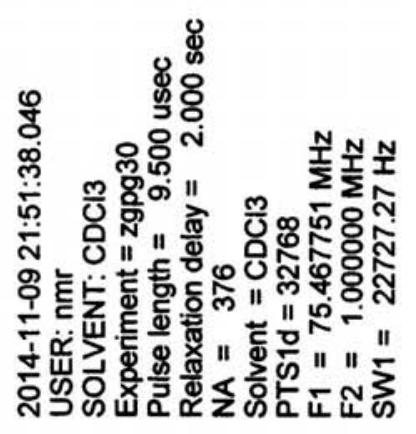

$6 \angle 9261$

$6 \varepsilon 0^{\circ} 812$

ণั่

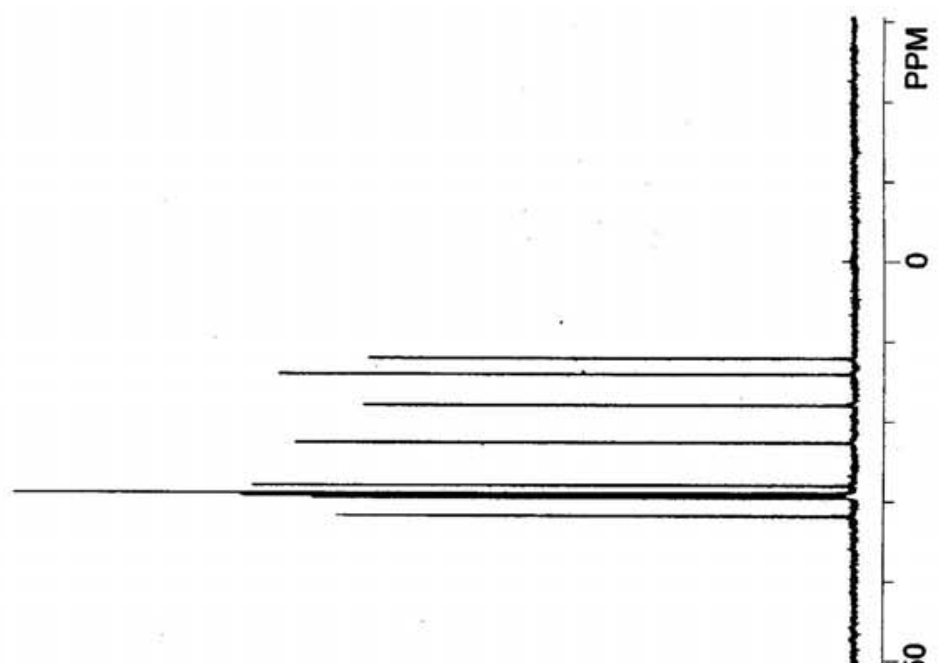

웅

옴

유

怘 

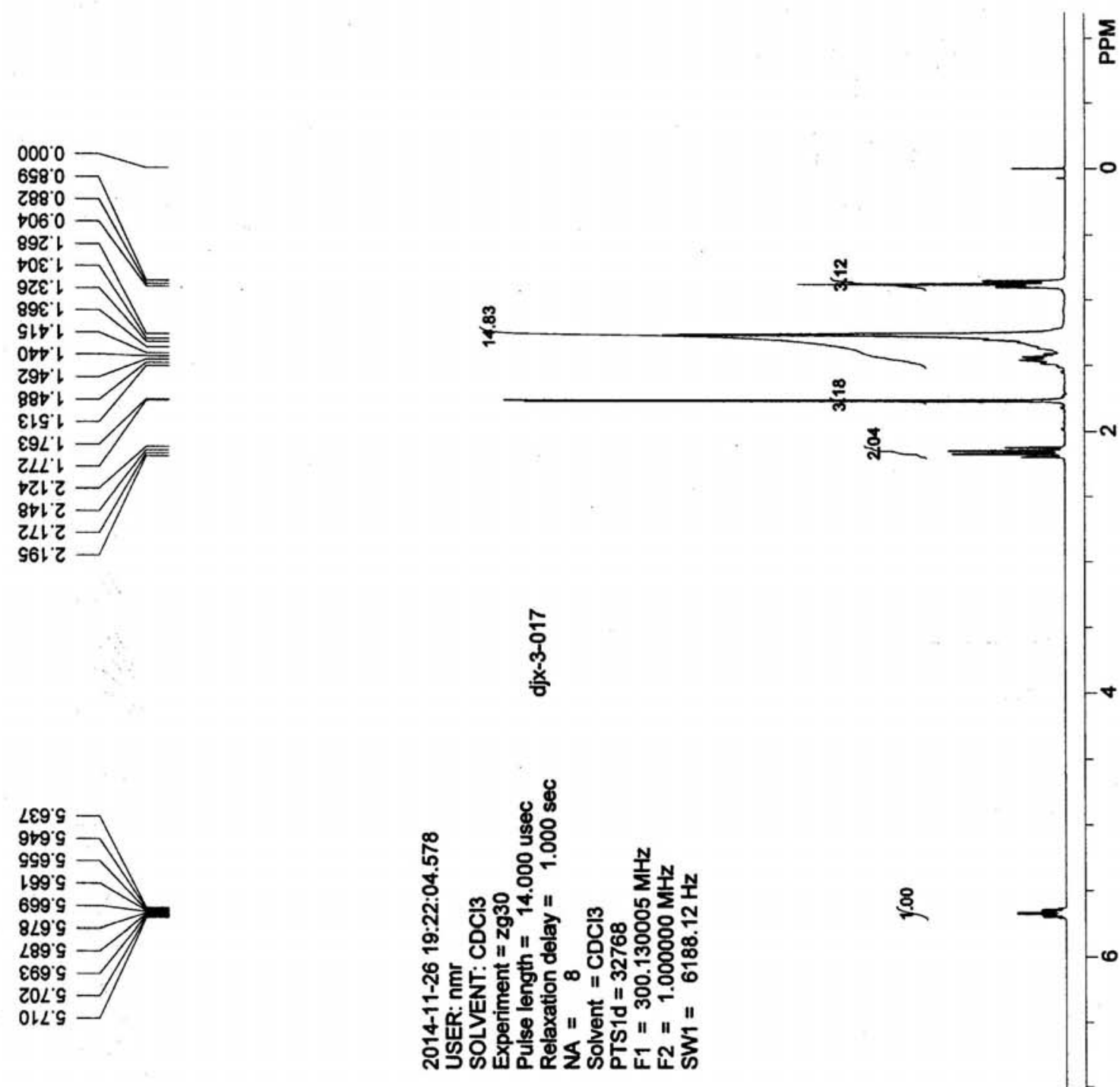

$\varepsilon 8 Z^{\prime} L$

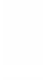



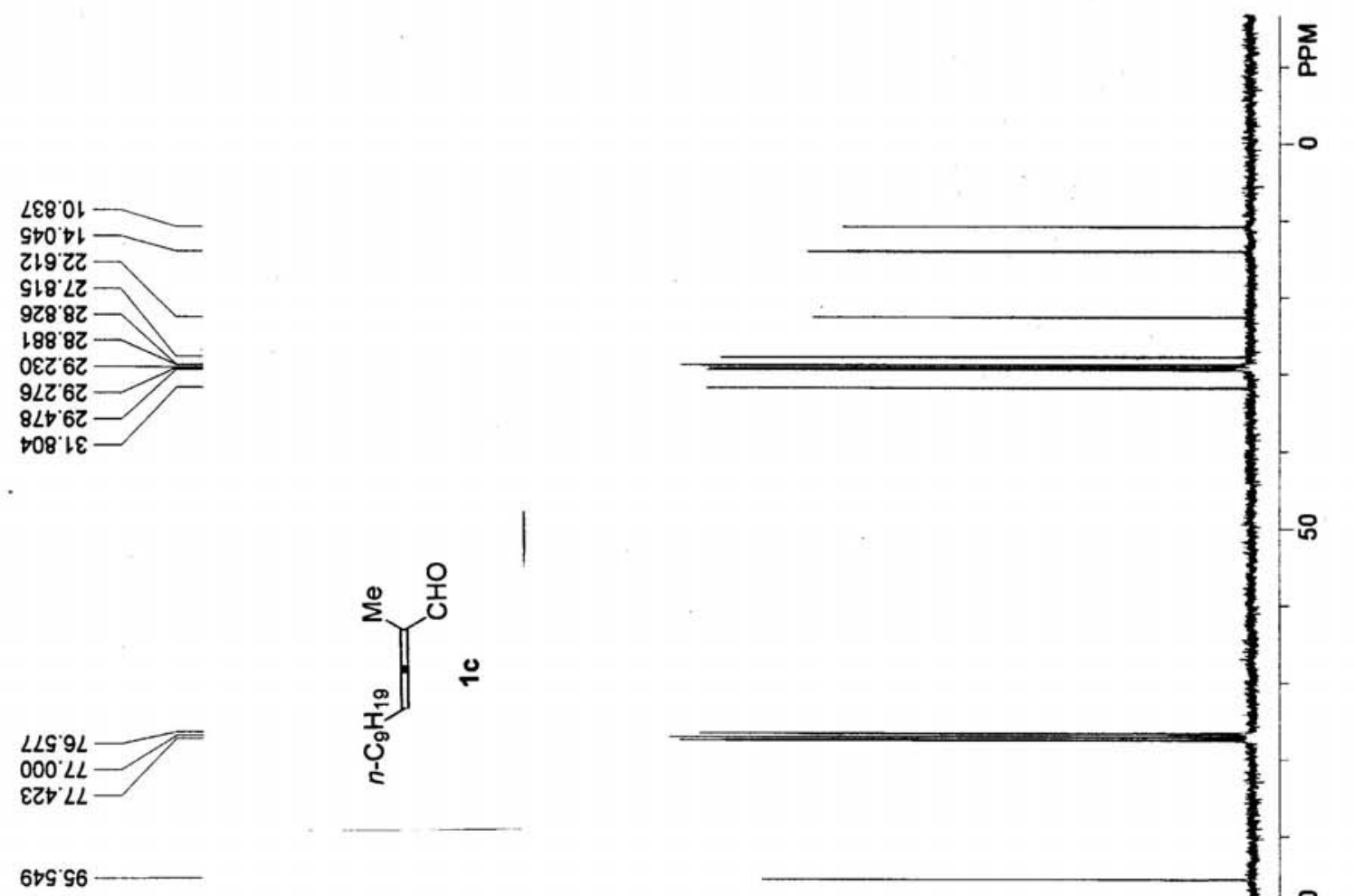

$89 \varepsilon^{\circ} 901$

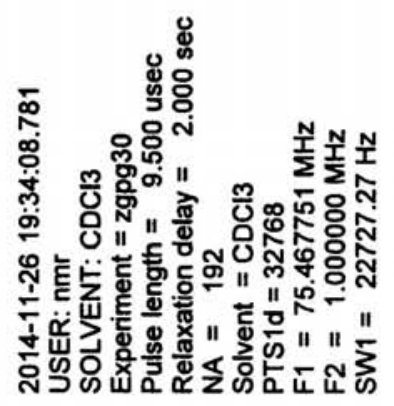

동

$610^{\circ} 861$

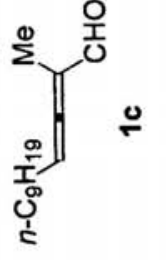

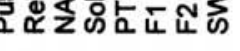

$1 \angle b 812$ 

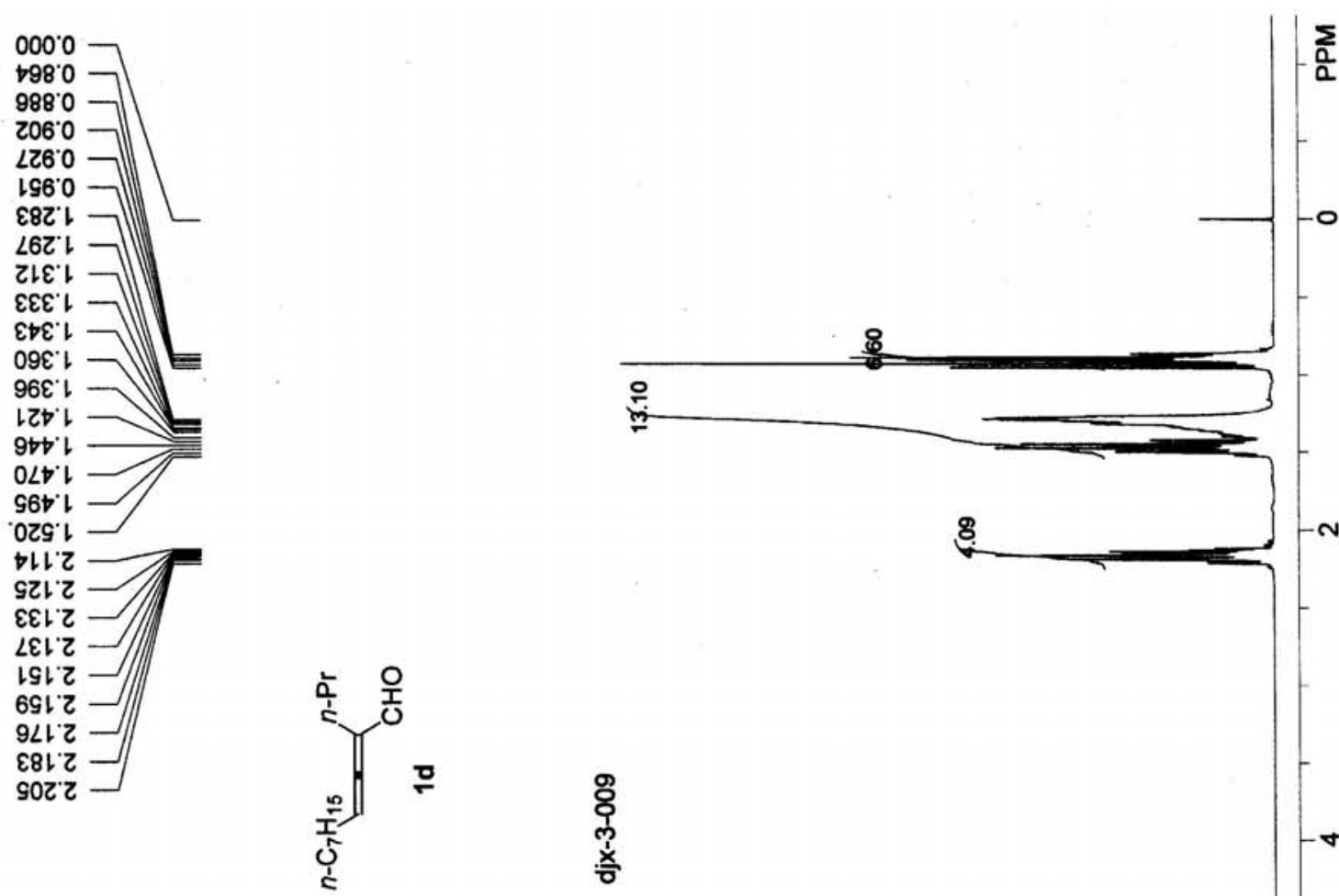

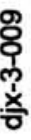
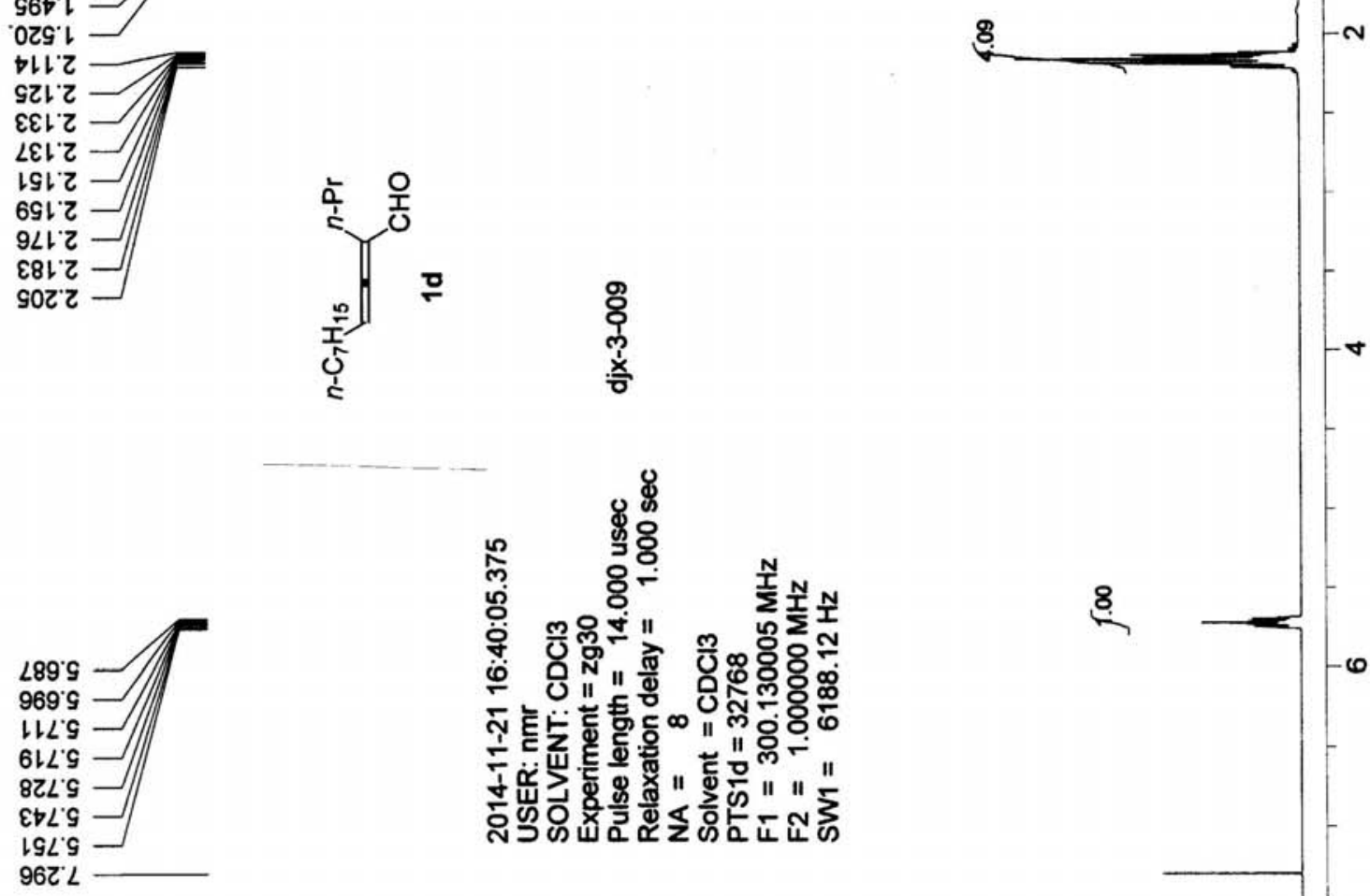

LLG' 6

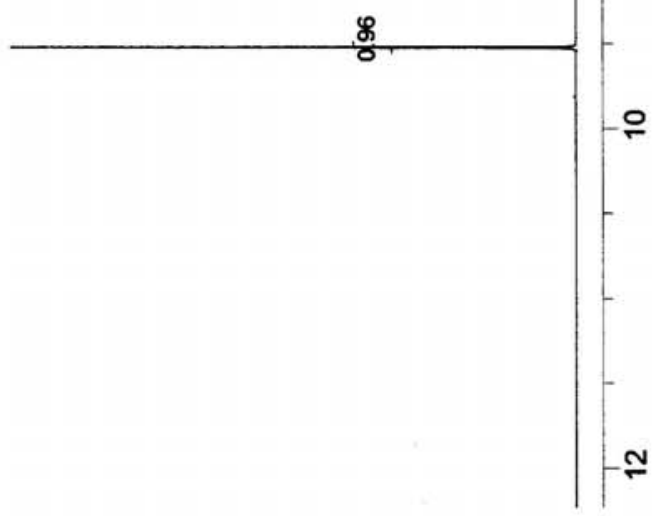



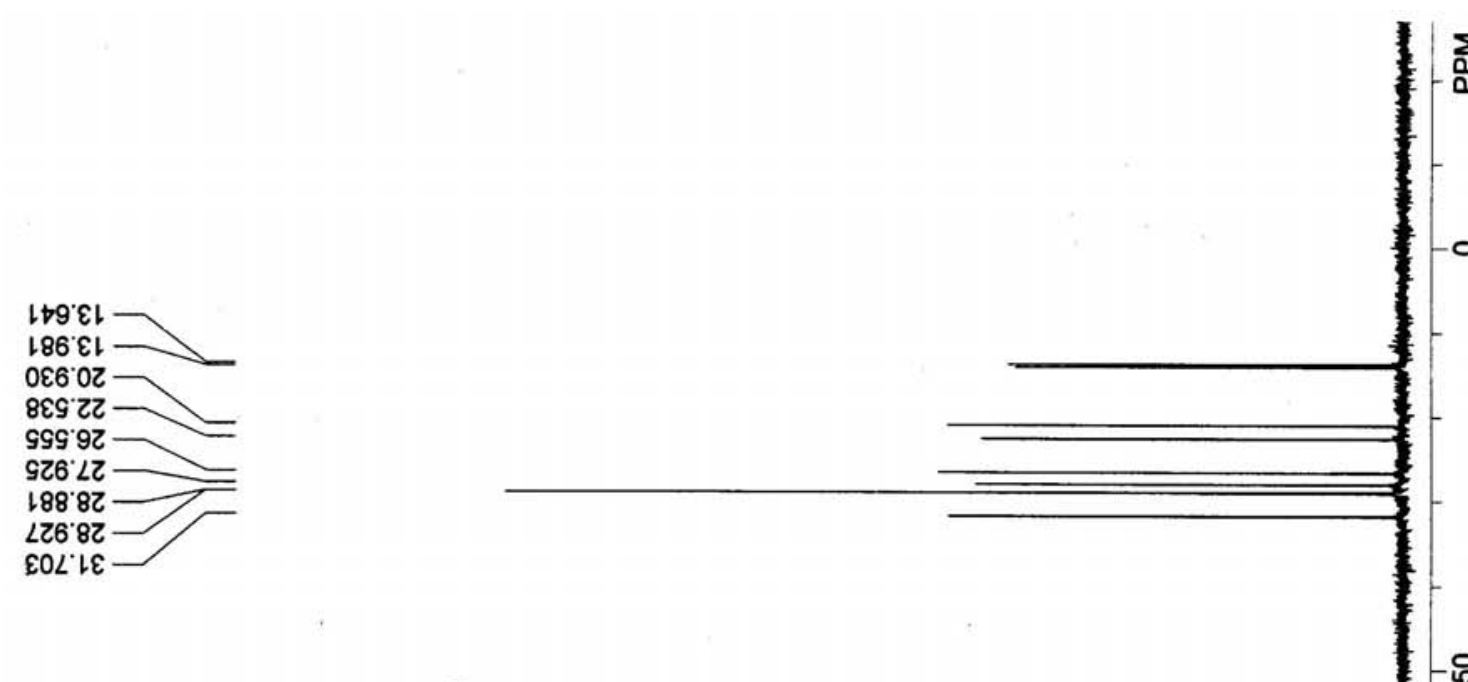

$\angle L G^{\prime} 9 L$

$000 . \angle L$

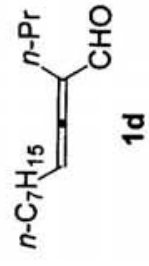

$\rightarrow 0 t 96$

옹
गे
ํㅡㅁ

09เレレー

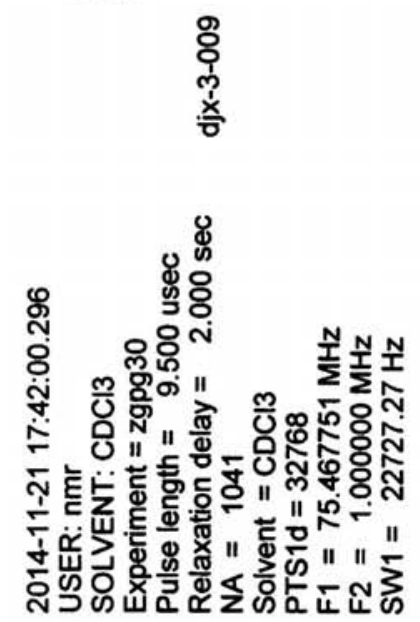

LLL'Z6L

$t+812$ 

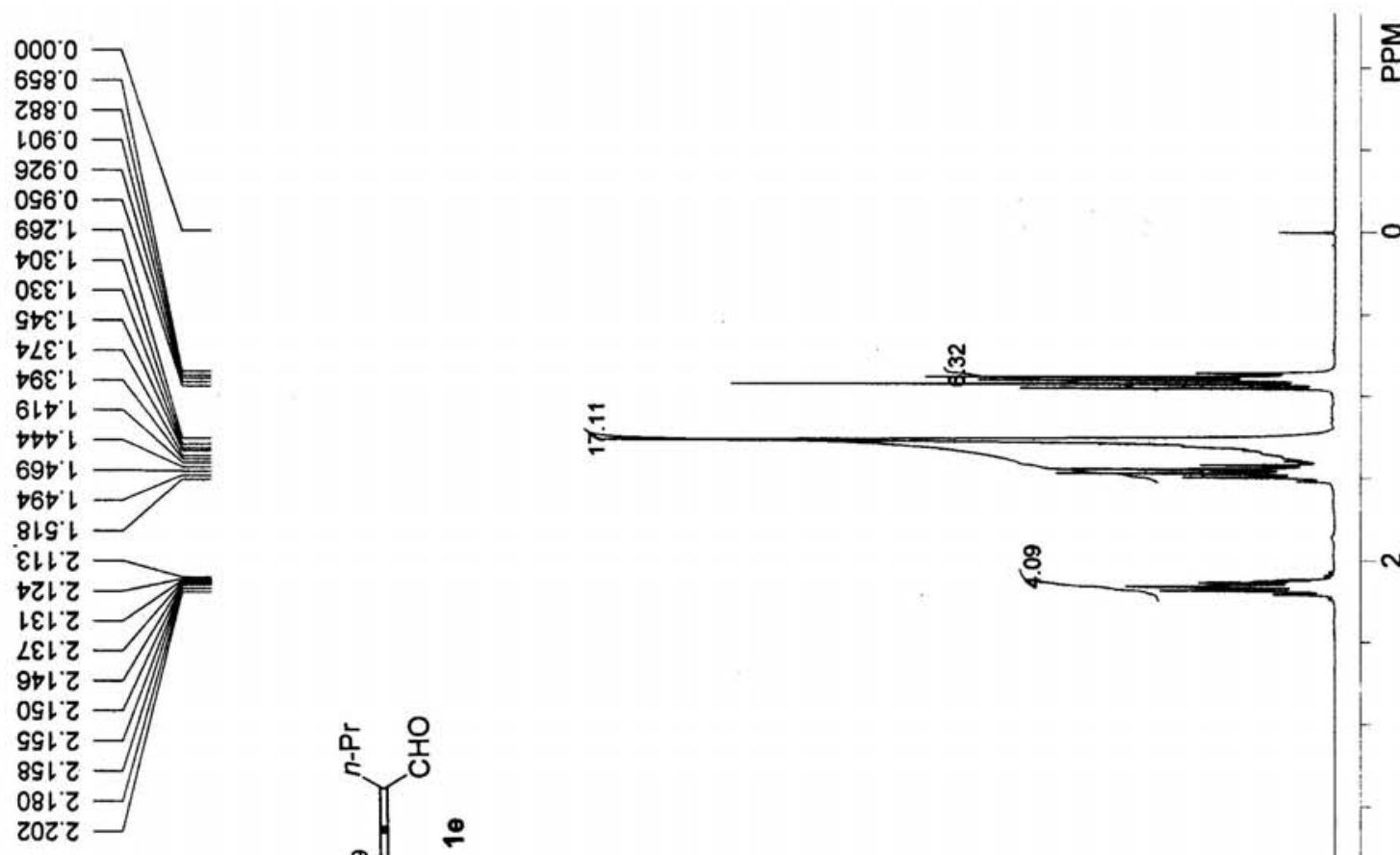

०६'

$\rightarrow \angle \varepsilon^{\circ}$
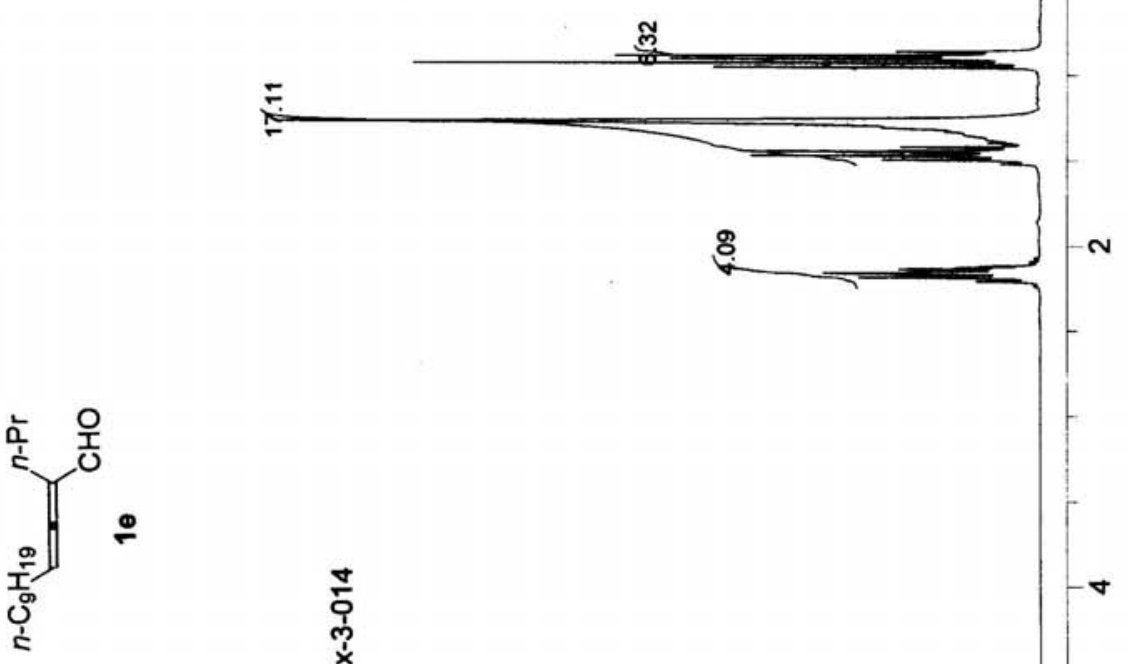

989' 9

$\varepsilon 69^{\circ} \mathrm{s}$

ZOL'

$80 \angle 9$

$\angle L L S$

SZL'

IEL'S

$0 t L ' S$

ot 1

$\forall 6 Z^{\prime} L$

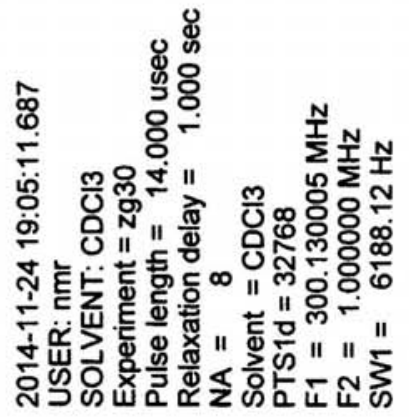

8 

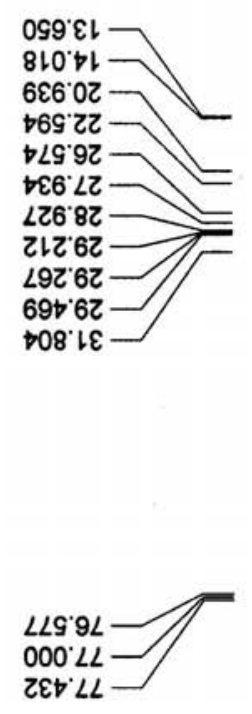

ยเจ 96

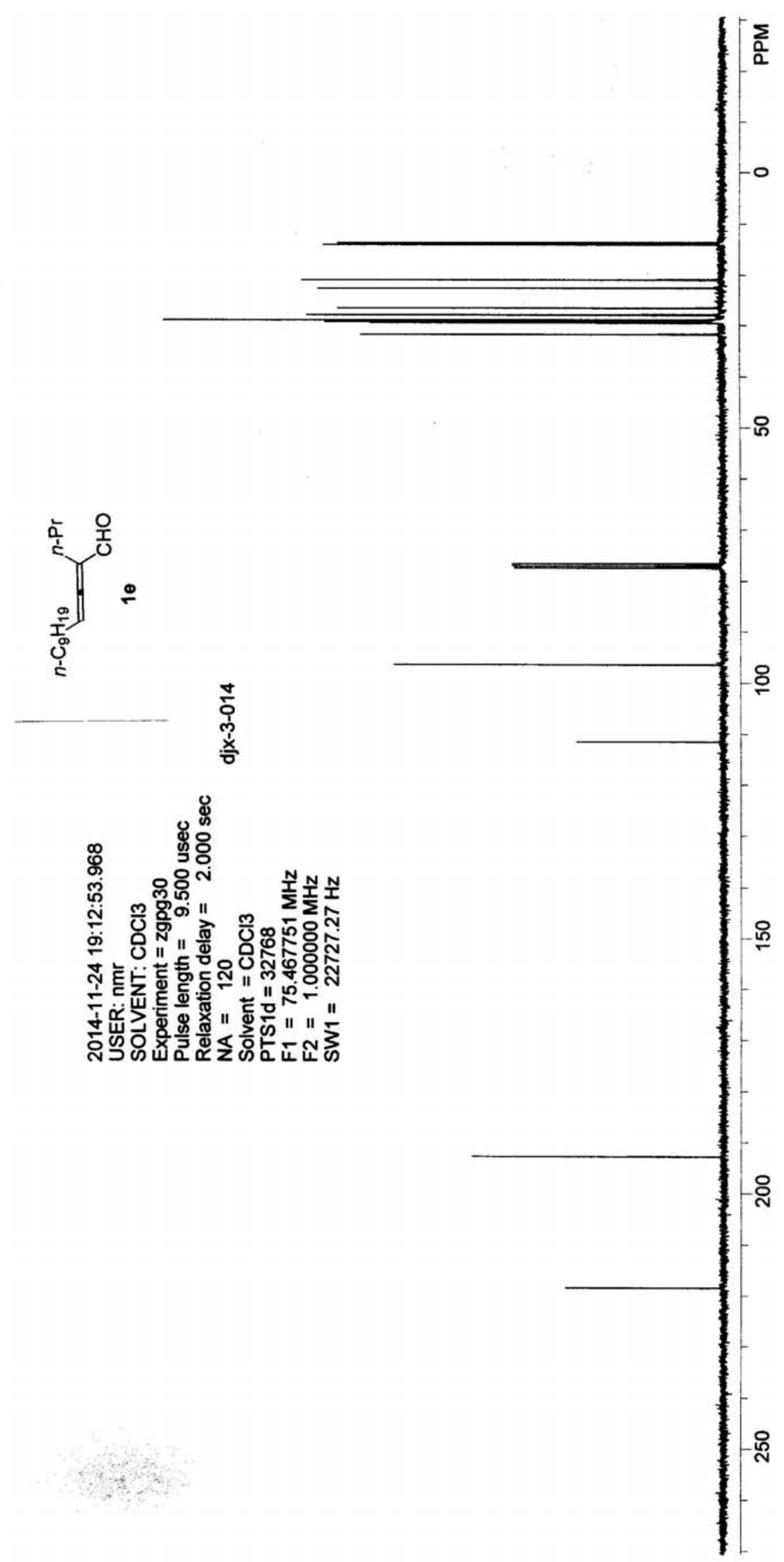

69เレルー

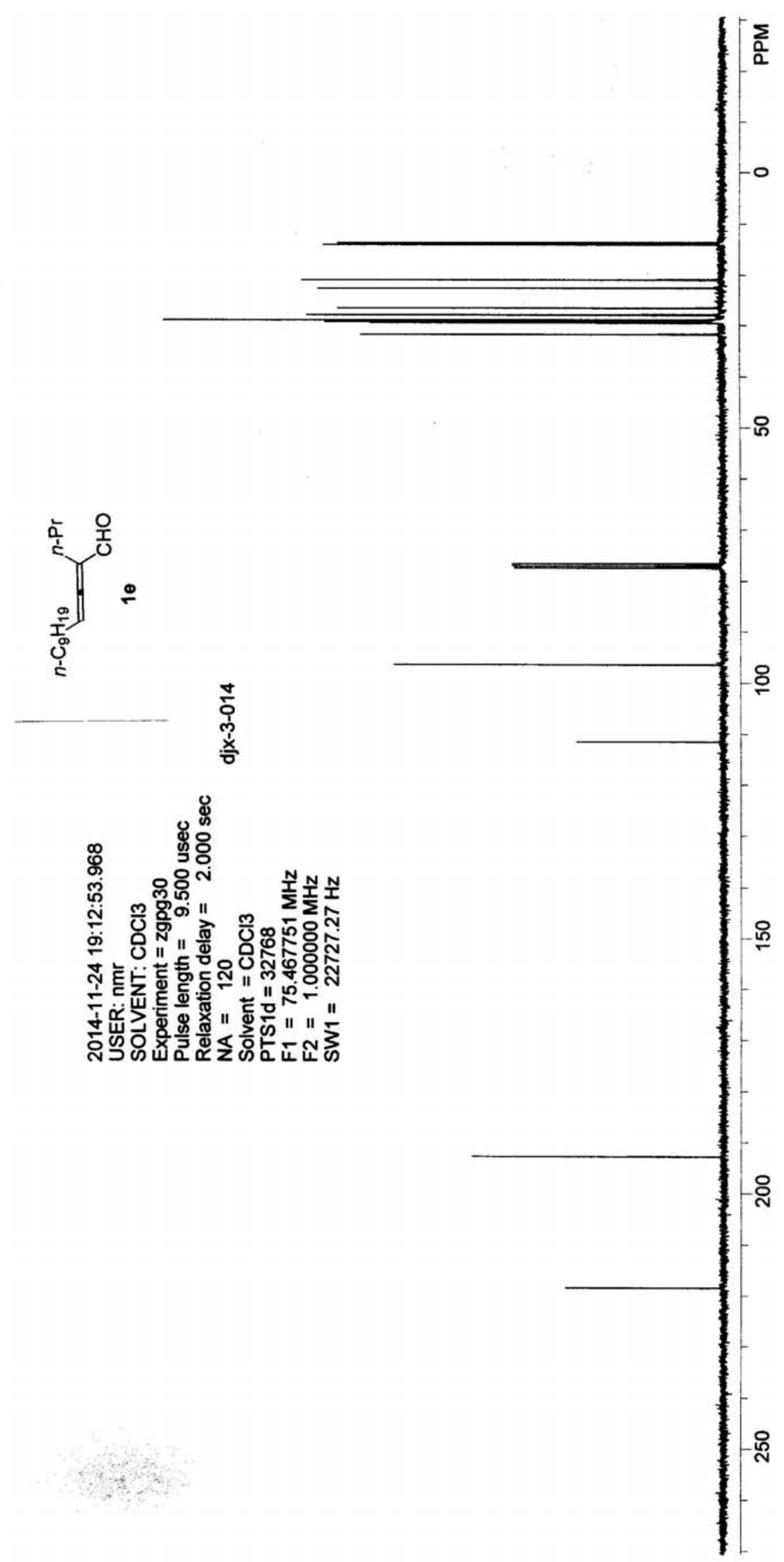

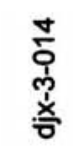

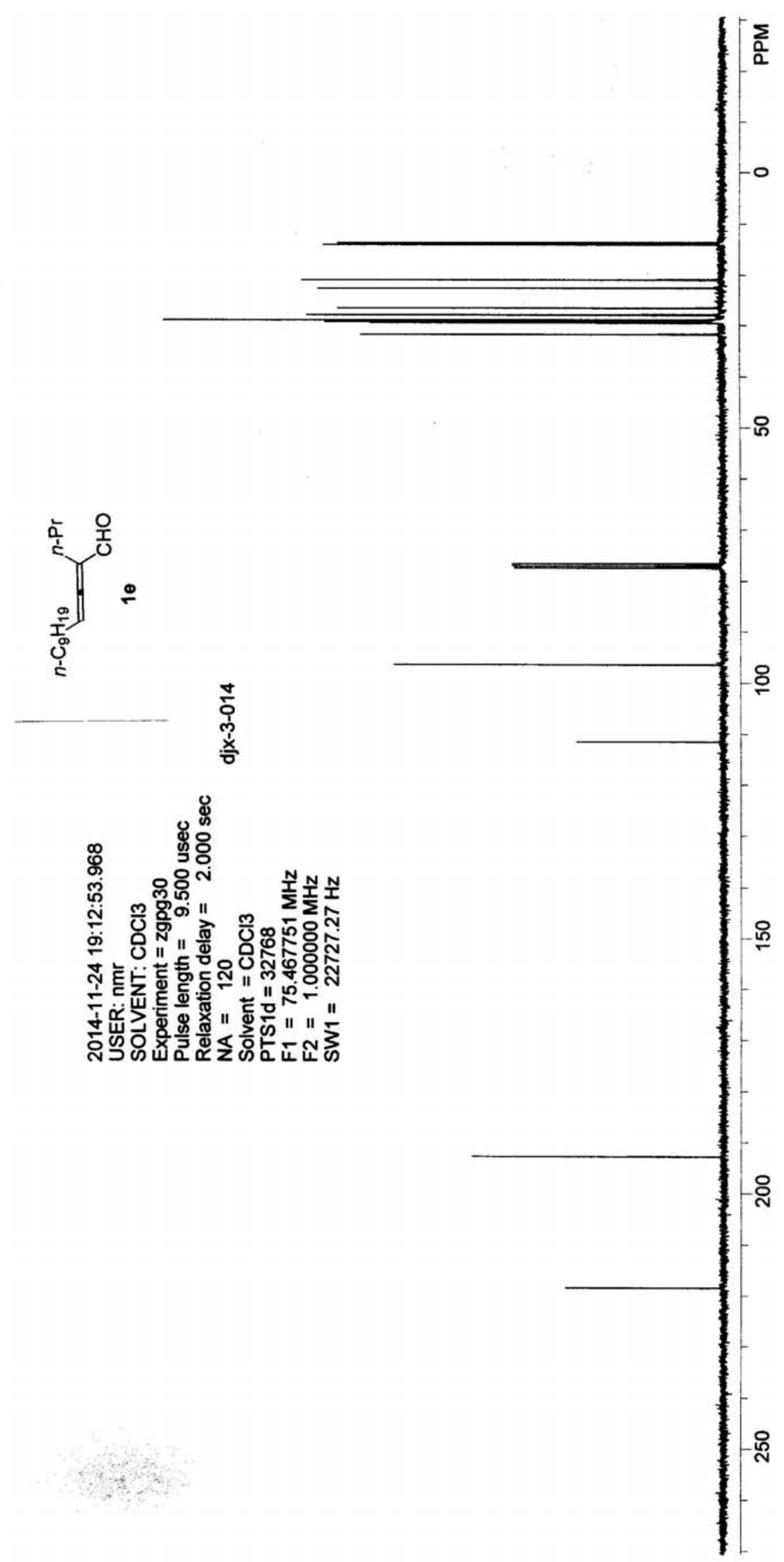

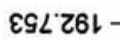

$+612$ 

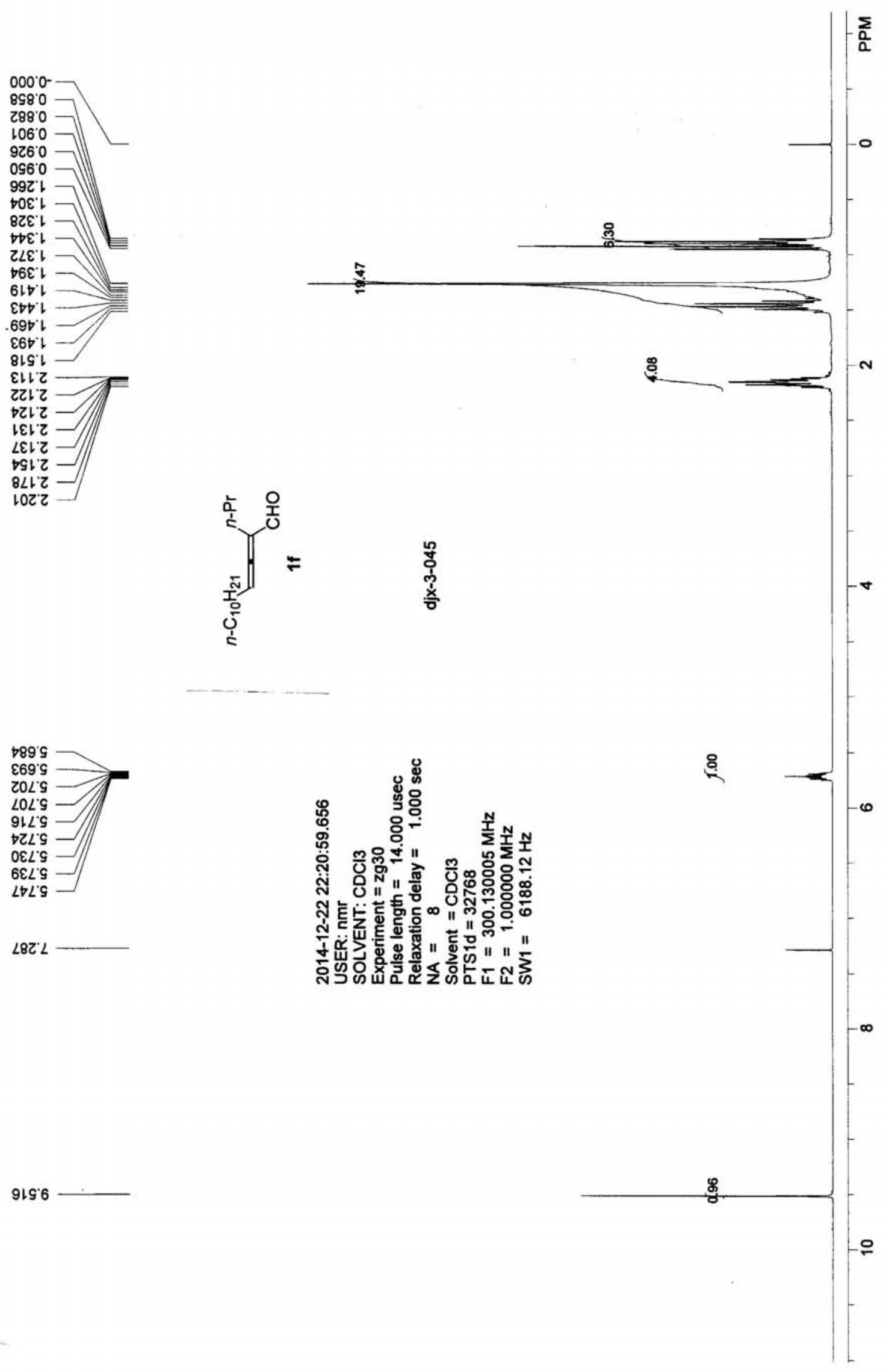

9เง 6 

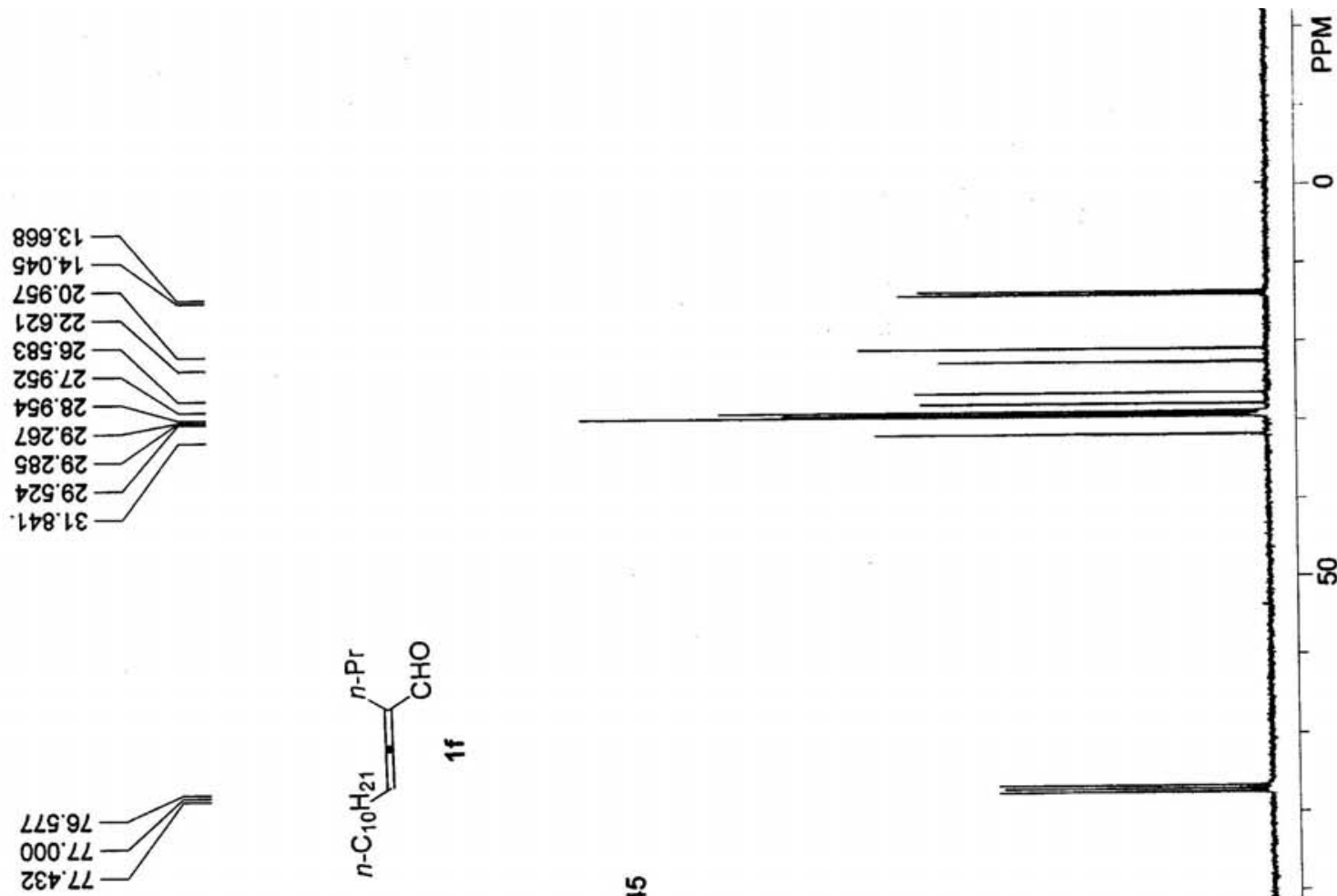

$2 Z \succ^{\prime} 96-$

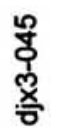

88 เレ乚

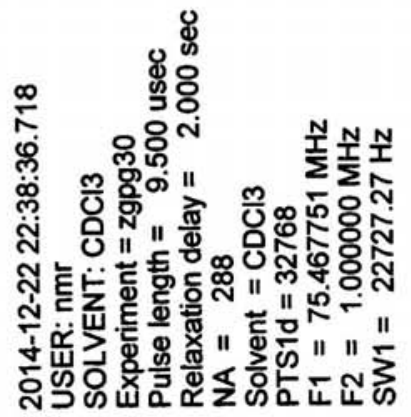

$68 L ' 26 L$

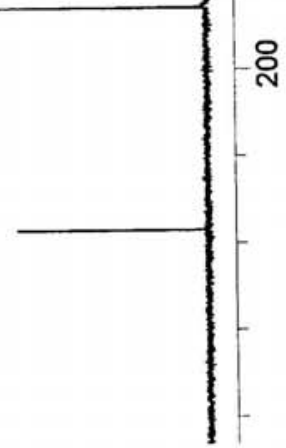



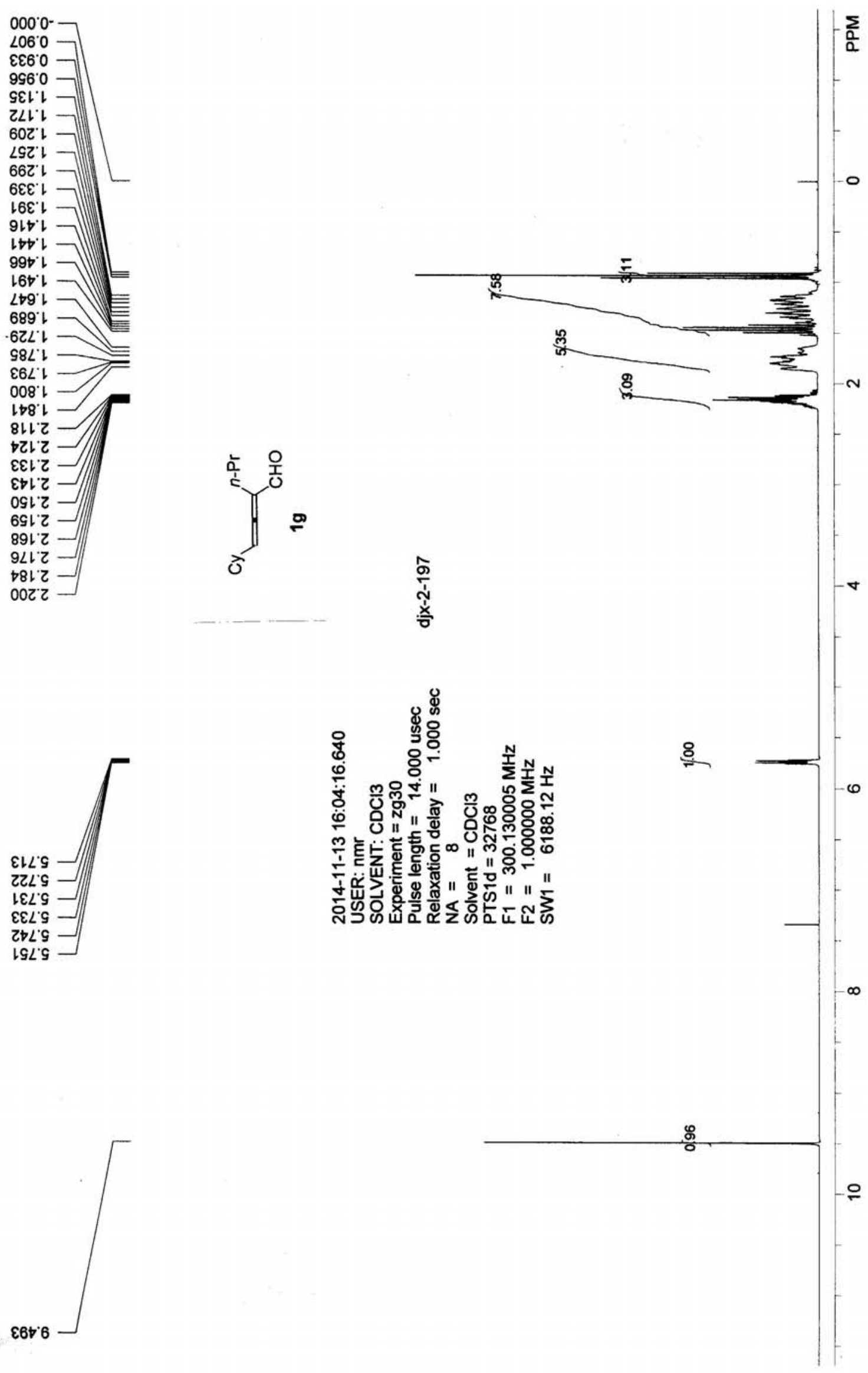

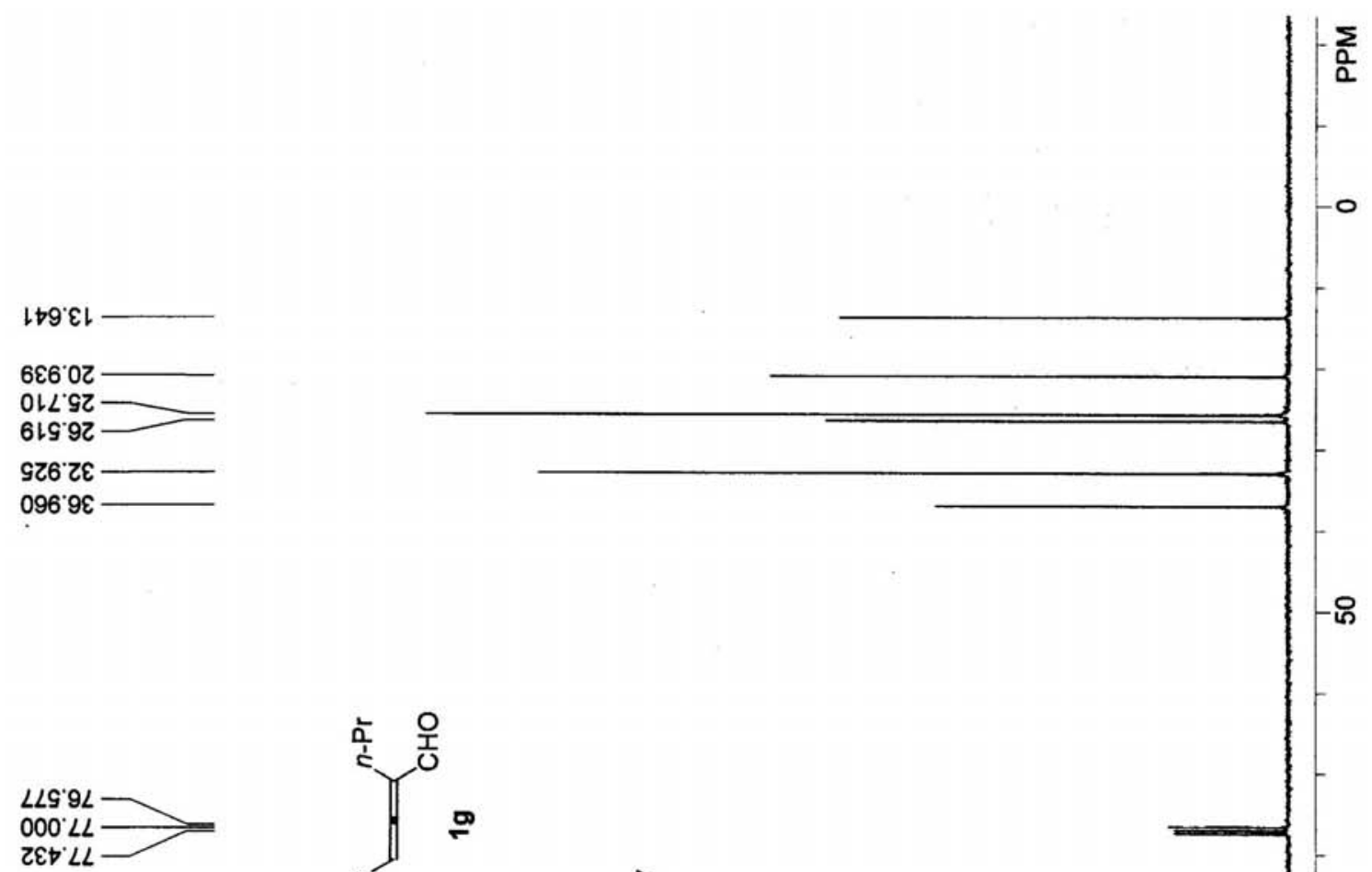<smiles>CC=CC(=CCO)CC</smiles>

ZZZ ZOL -

๕9ャてレ

乙๕૬'乙6เ -

$\forall \forall 9<12$<smiles>[Ge]=C=[Ge]</smiles>

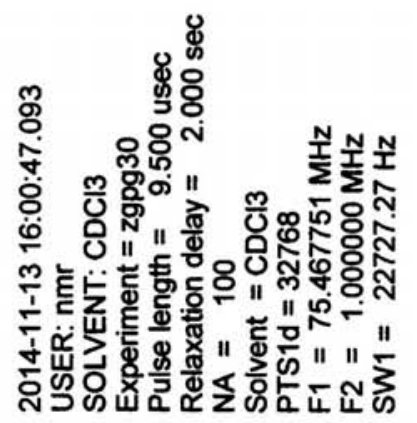

무

衣

움

$\stackrel{2}{\circ}$

- 

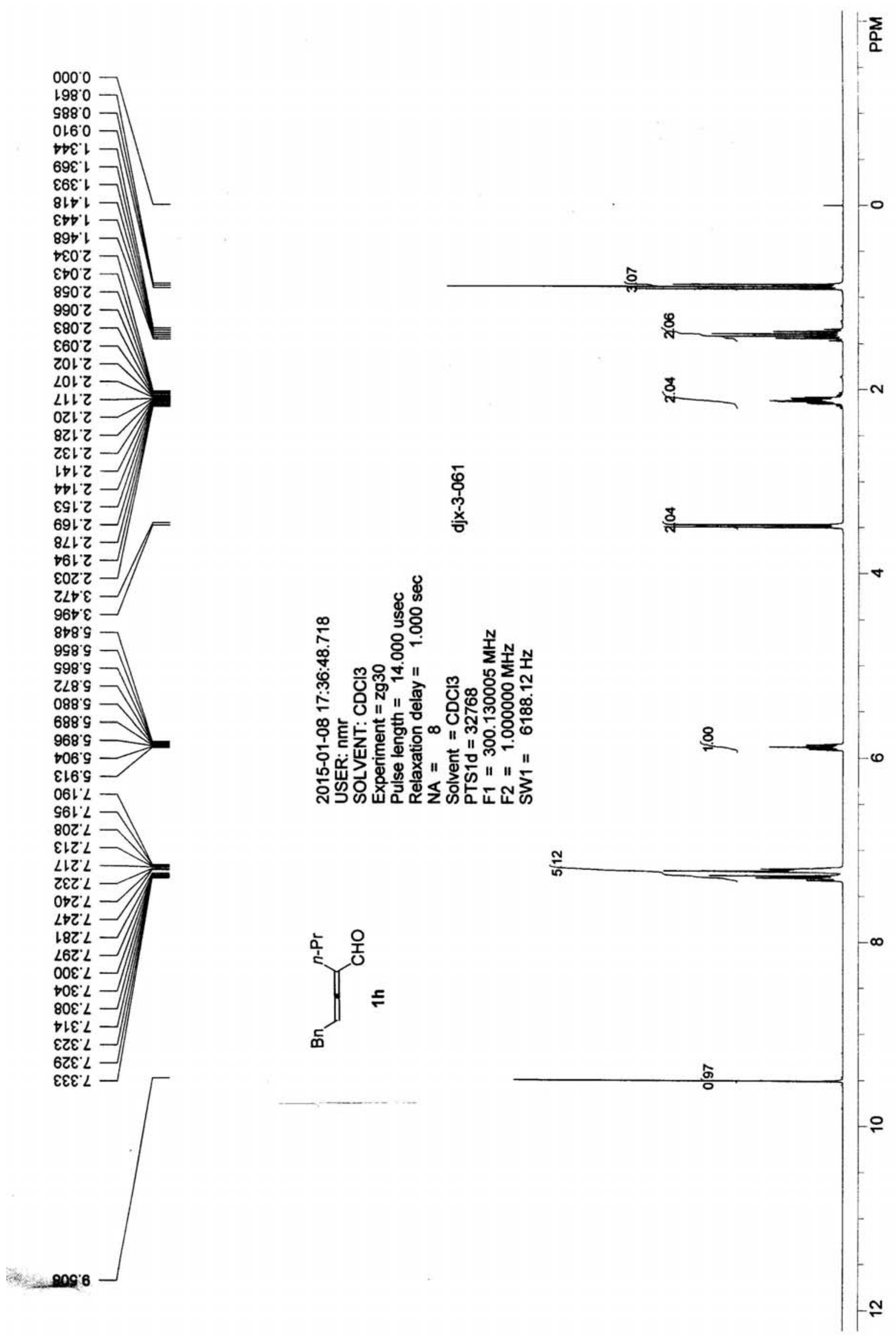


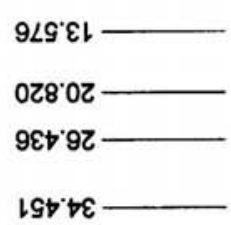

LLG' $9 L$

$000 \%$

$\varepsilon Z \succ L L$

S9l. 96

StLันเ

069 $9 Z$ Z

$66 Z$ ' $8 Z$ L

ह8॰ 821

เZS' $8 \varepsilon$ ।

99Z Z6เ

819.812

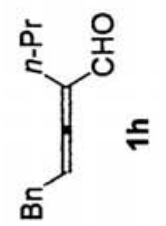

$\bar{y}$
ํ.
x.
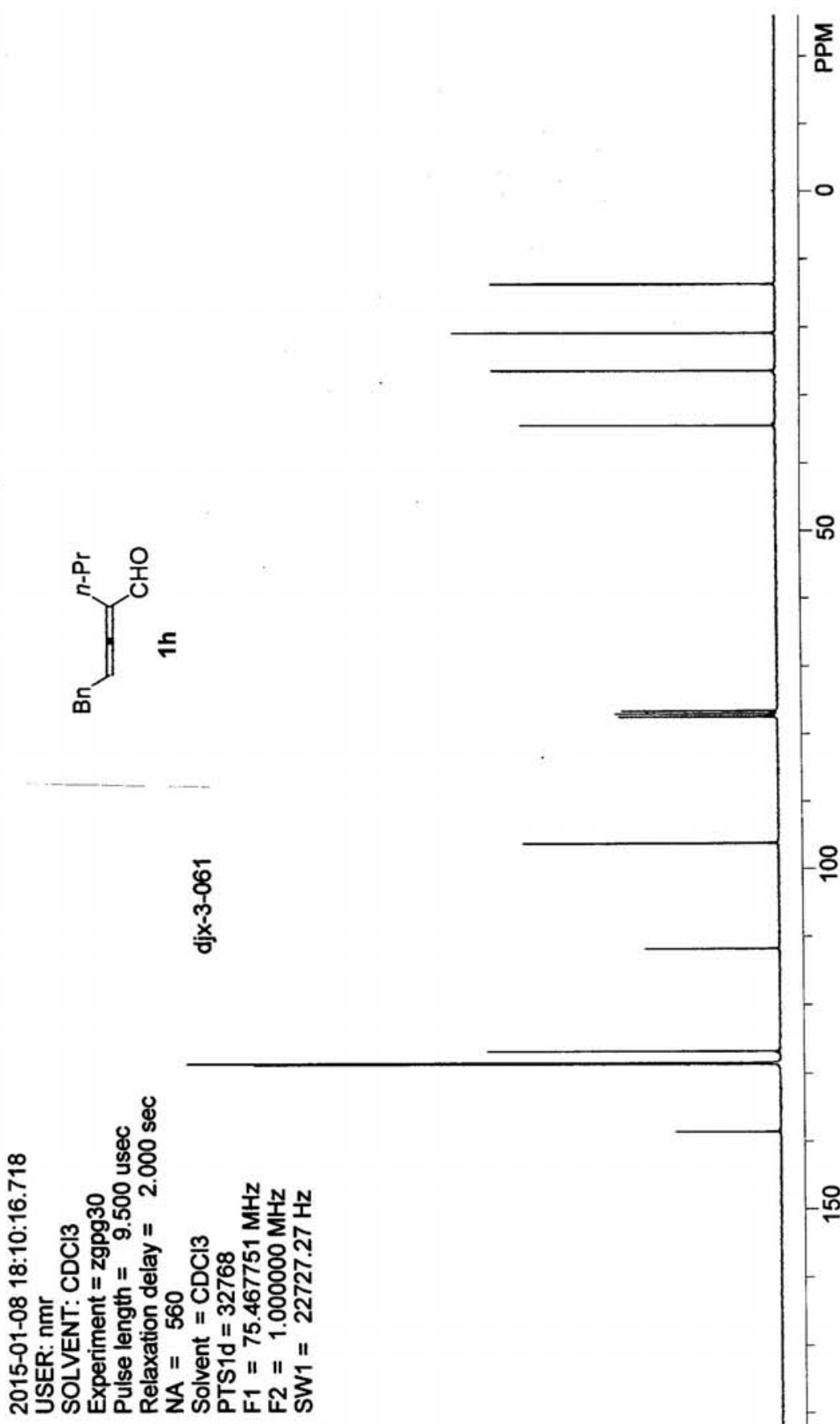

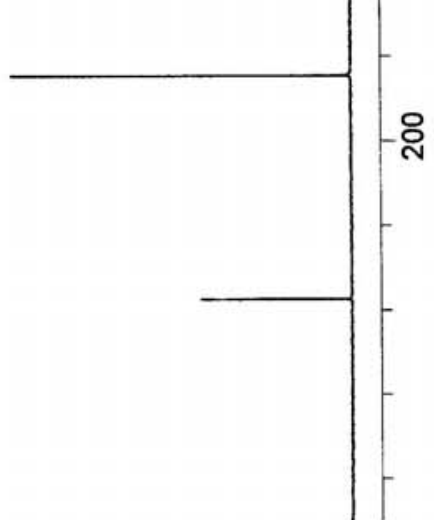



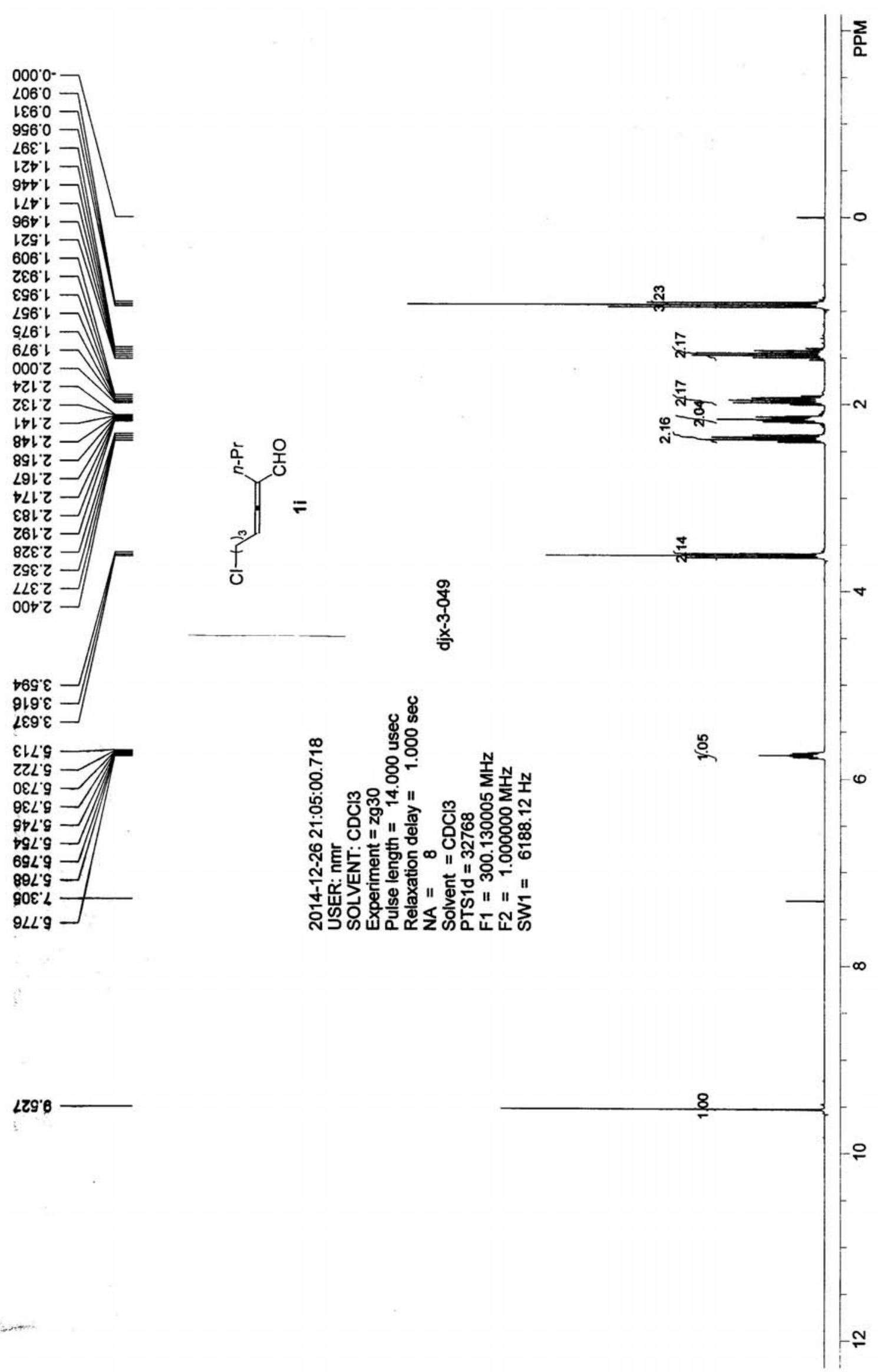

LZQ' 6

EL'S

$Z Z L S$

$\theta \varepsilon L^{\prime}$

$97 L ' S$

tQL'

$69<$ ' 9

$89 L^{\circ}$

$9 L 29$

$-1$

今 8

8

$\sum^{N} N$ T $N$

잉

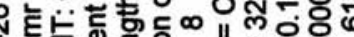

交

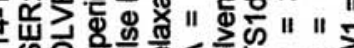

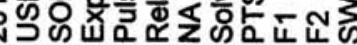



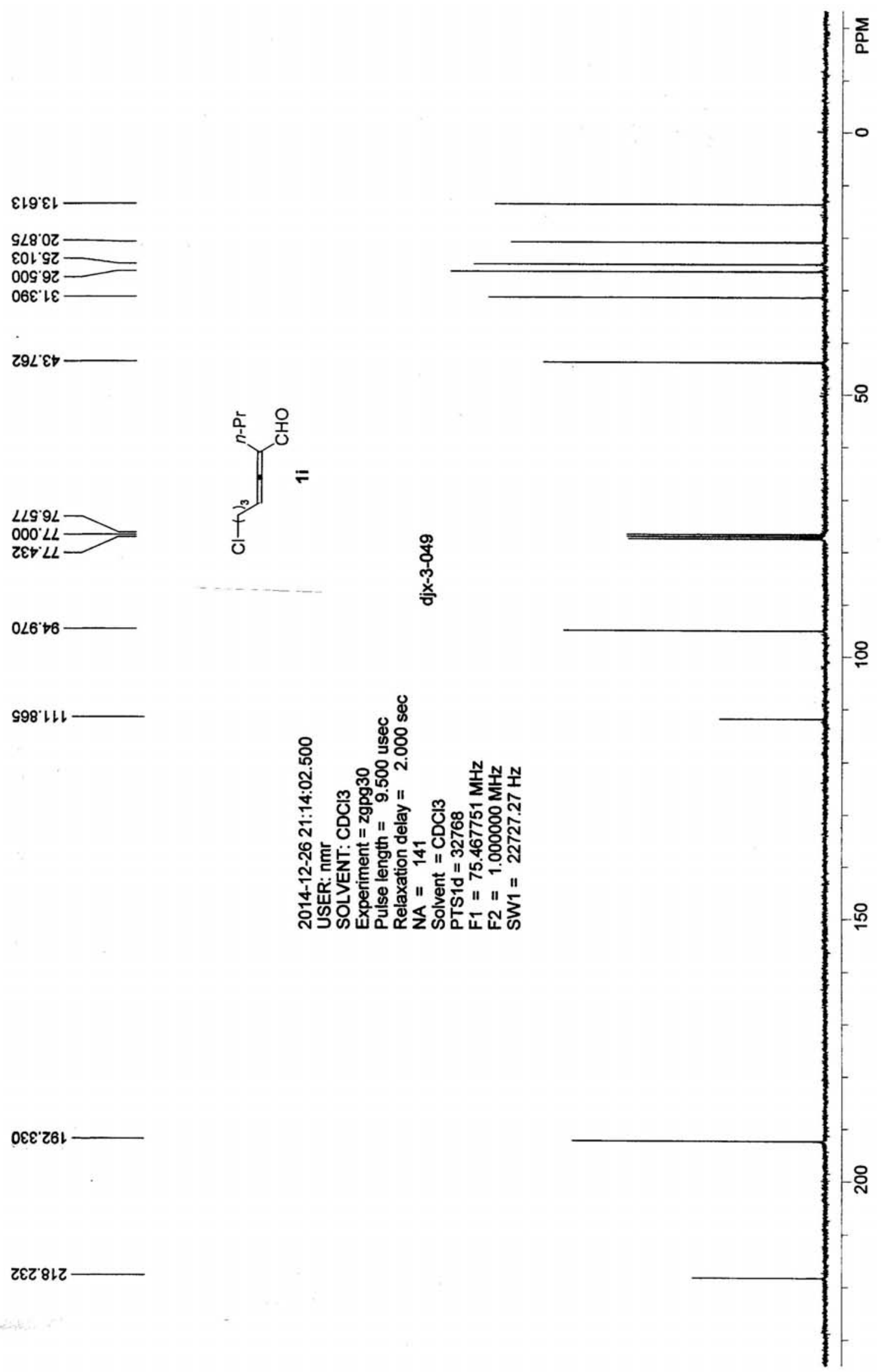

乙๕乙 $8 เ 乙$

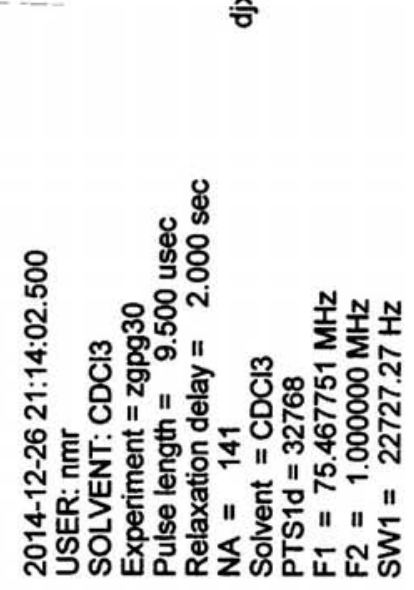

o๕๕'z६เ

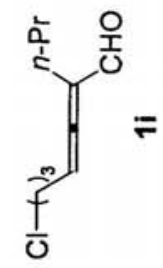

ZET $\angle L$

$0 \angle 6^{\circ} \rightarrow 6$

998เレ 


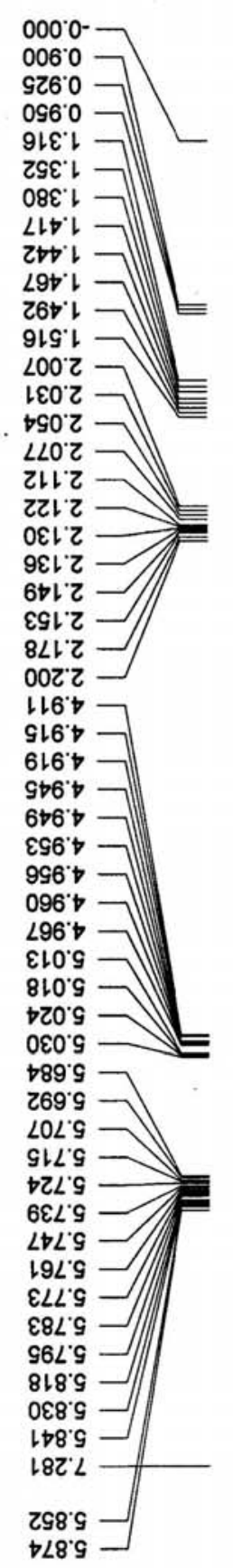

$\mid-\frac{2}{a}$

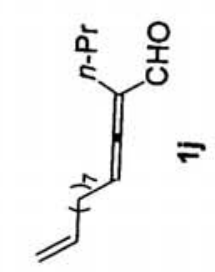

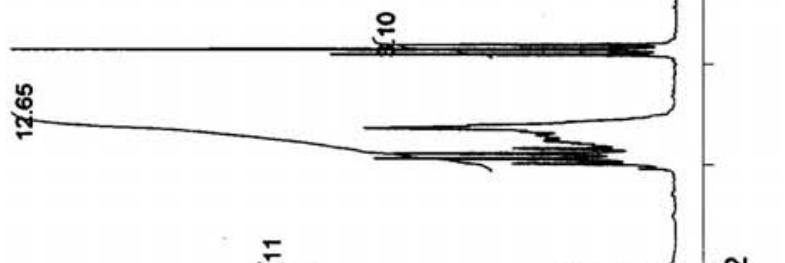

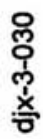
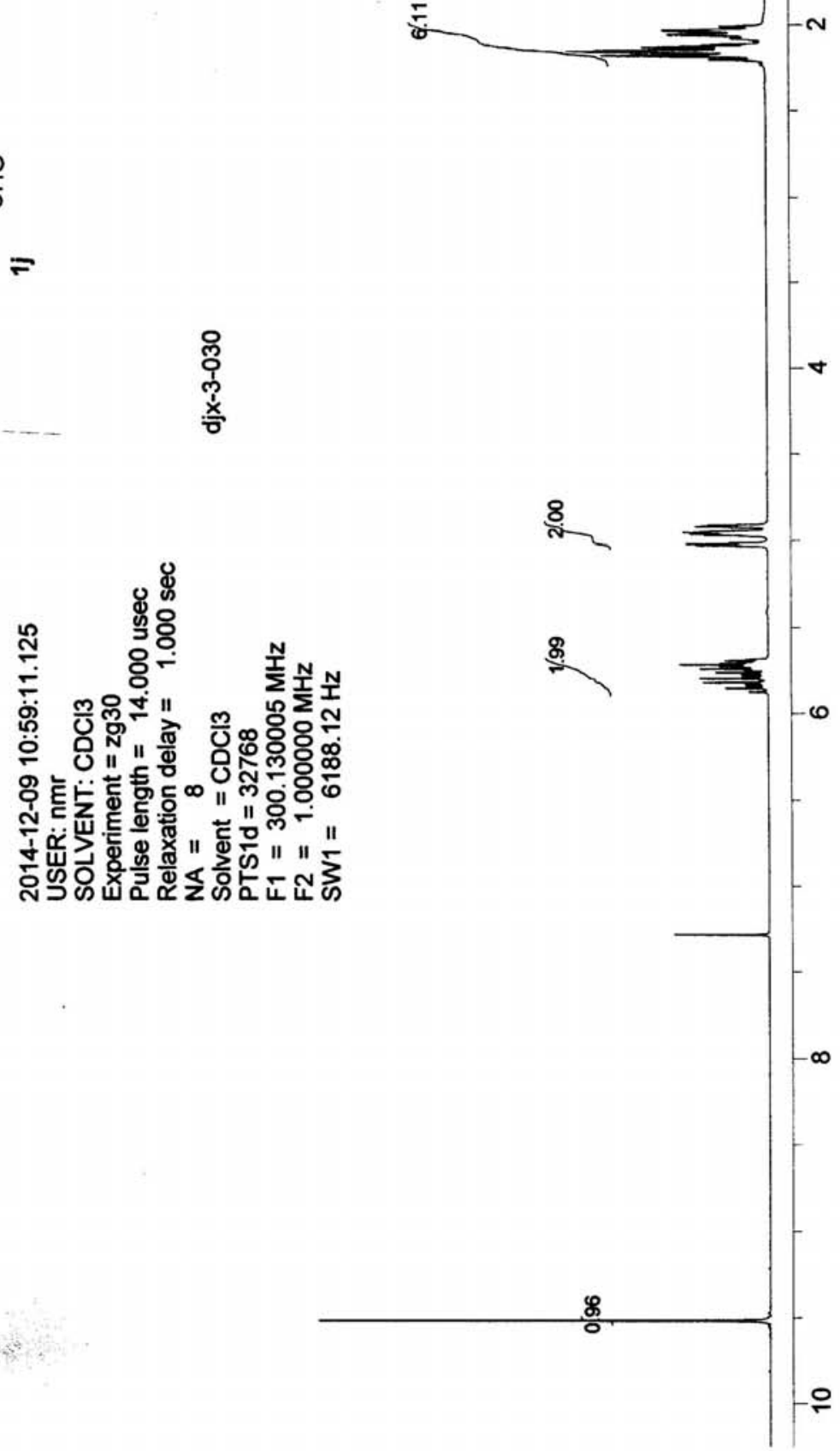

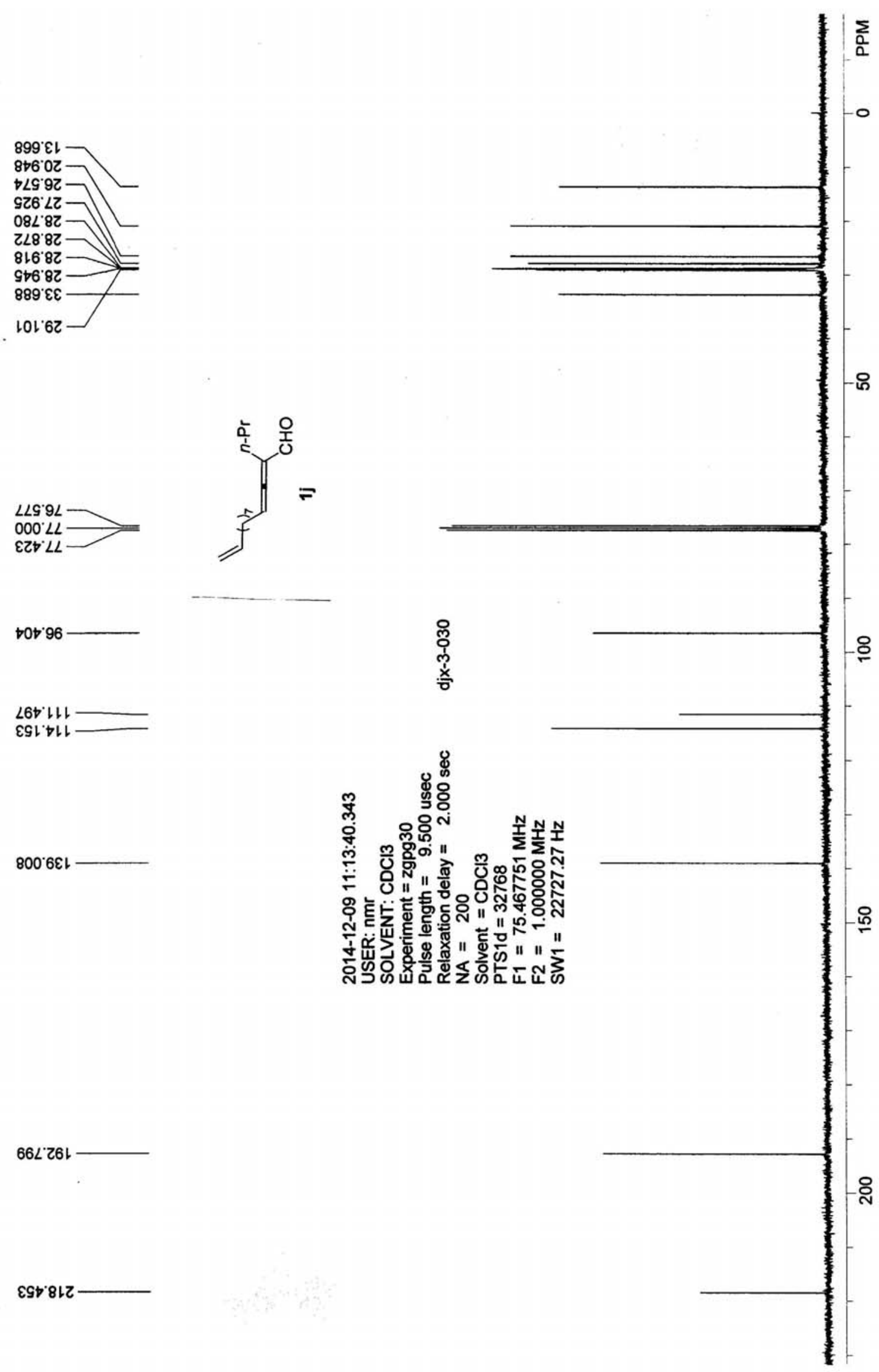

\&૬เ 8เZ -

$66 L^{\prime} Z 6 \vdash$

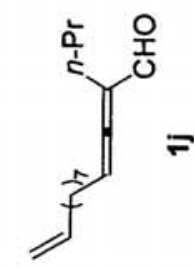

26t'เL

ESL $\rightarrow L L$

$800^{\circ} 6 \varepsilon\llcorner$ 

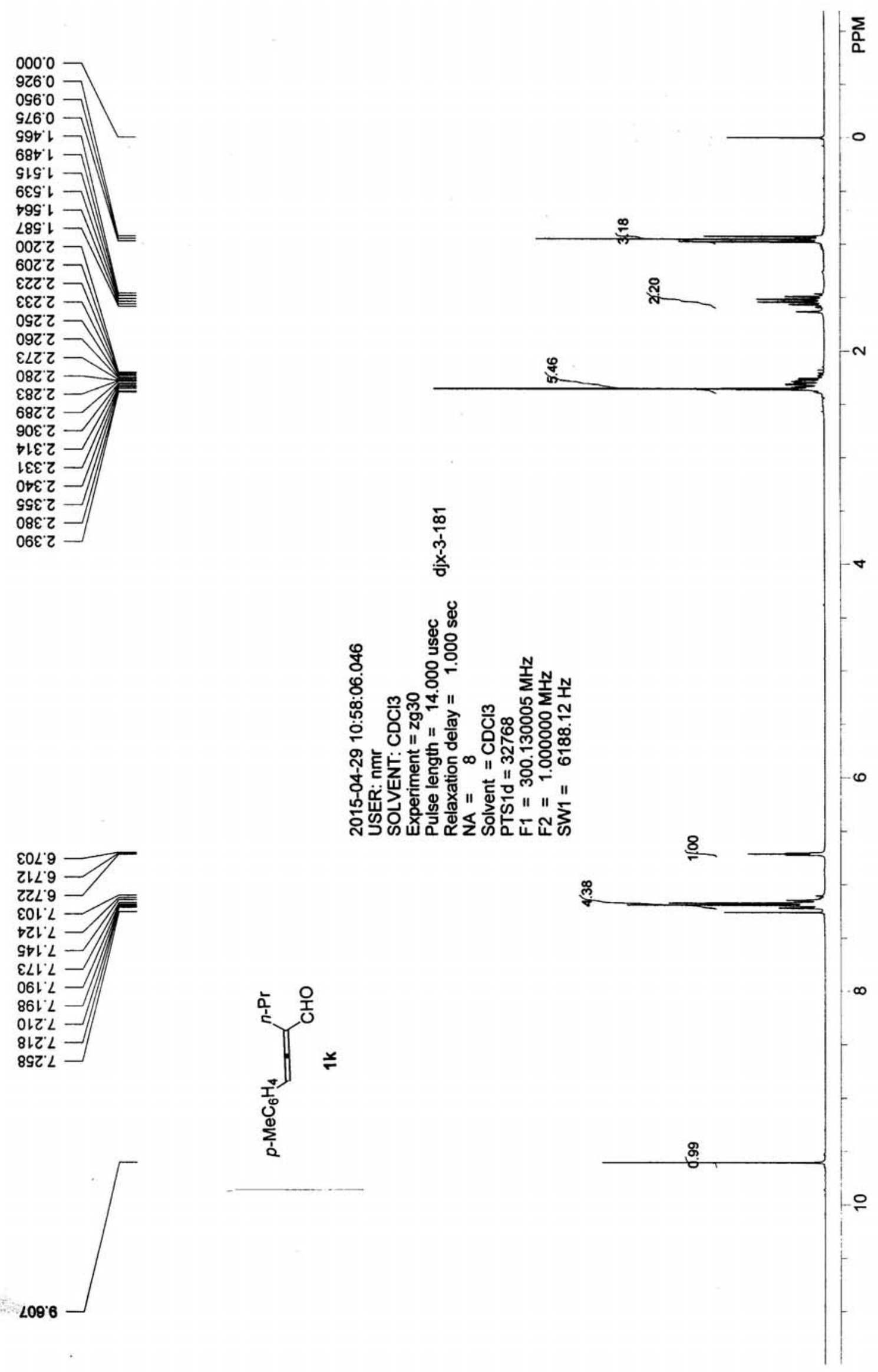
५ว८' $\varepsilon\llcorner$

890.12

ยะ乙 เ乙

$\checkmark 20^{\circ} \angle Z$

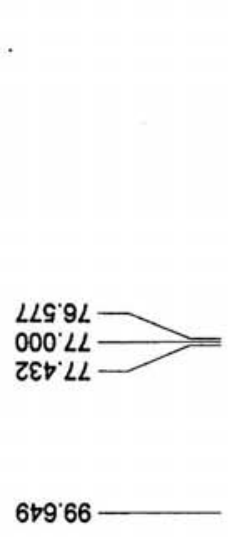

$688 \bullet เ レ$

ISL LZI

S97 82 I

७९ट $8 \varepsilon ।$

s89.0Z2

วะเ'เ6เ

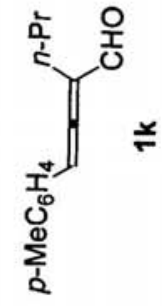

कृ

$\mid$

$\frac{5}{2}$

8

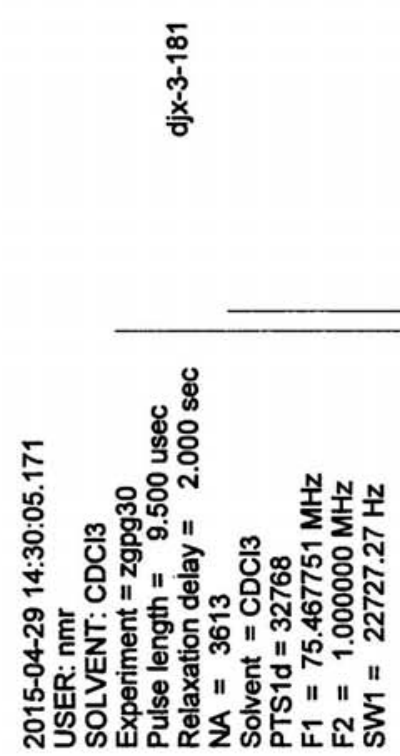

-

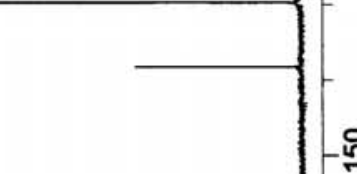



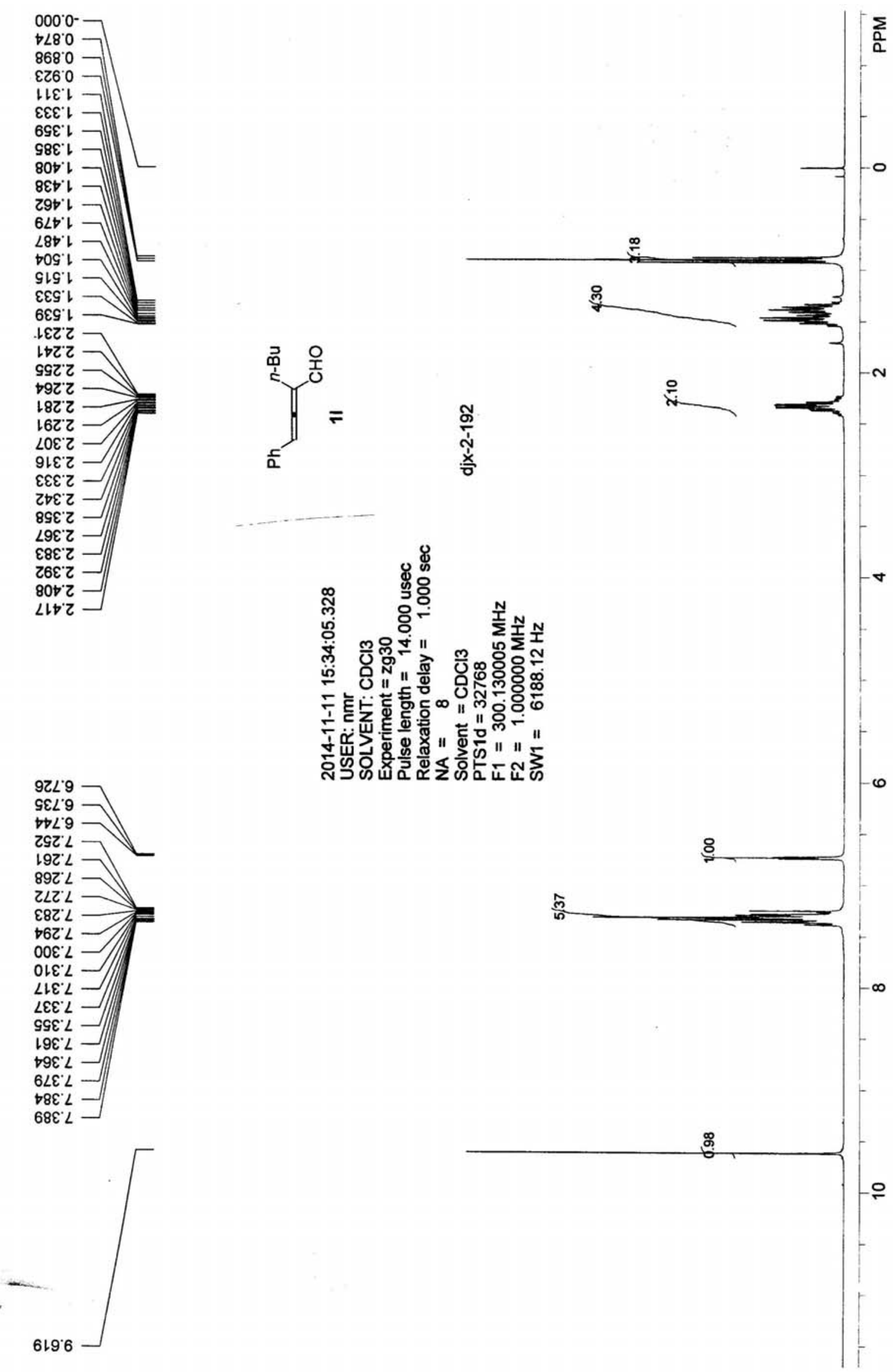

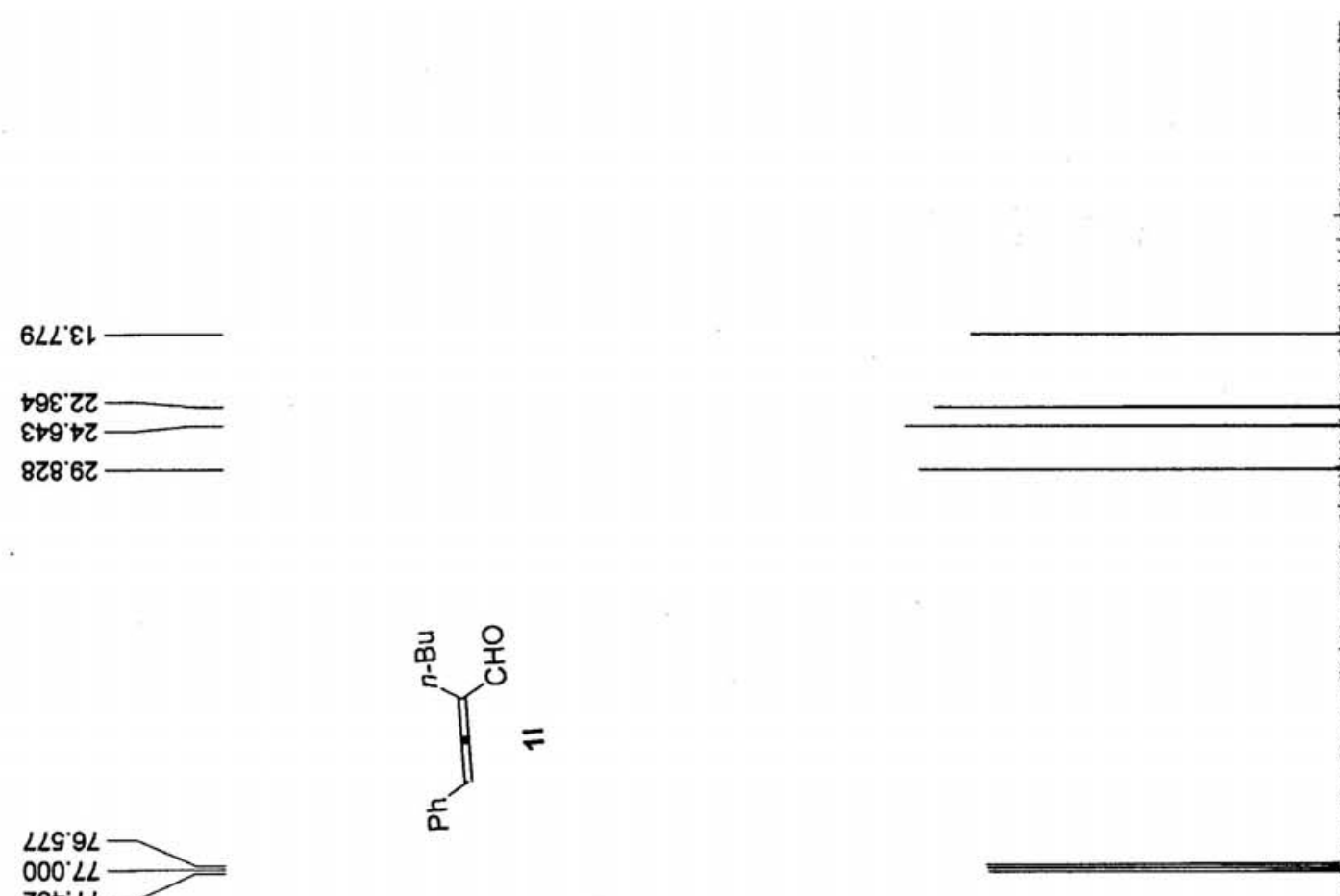

ZEเ $\angle L$

乙๖8 66

๙ั่

OOLSL

$90 Z \angle Z L$

081.821

\&†6. $8 \mathrm{CL}$

$\angle L G^{\prime}|\varepsilon|$

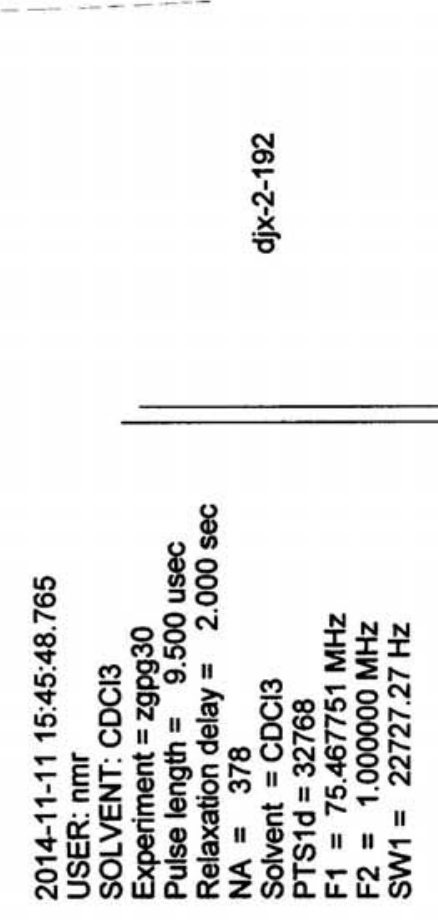

$989 \cdot 16 \downarrow$ 

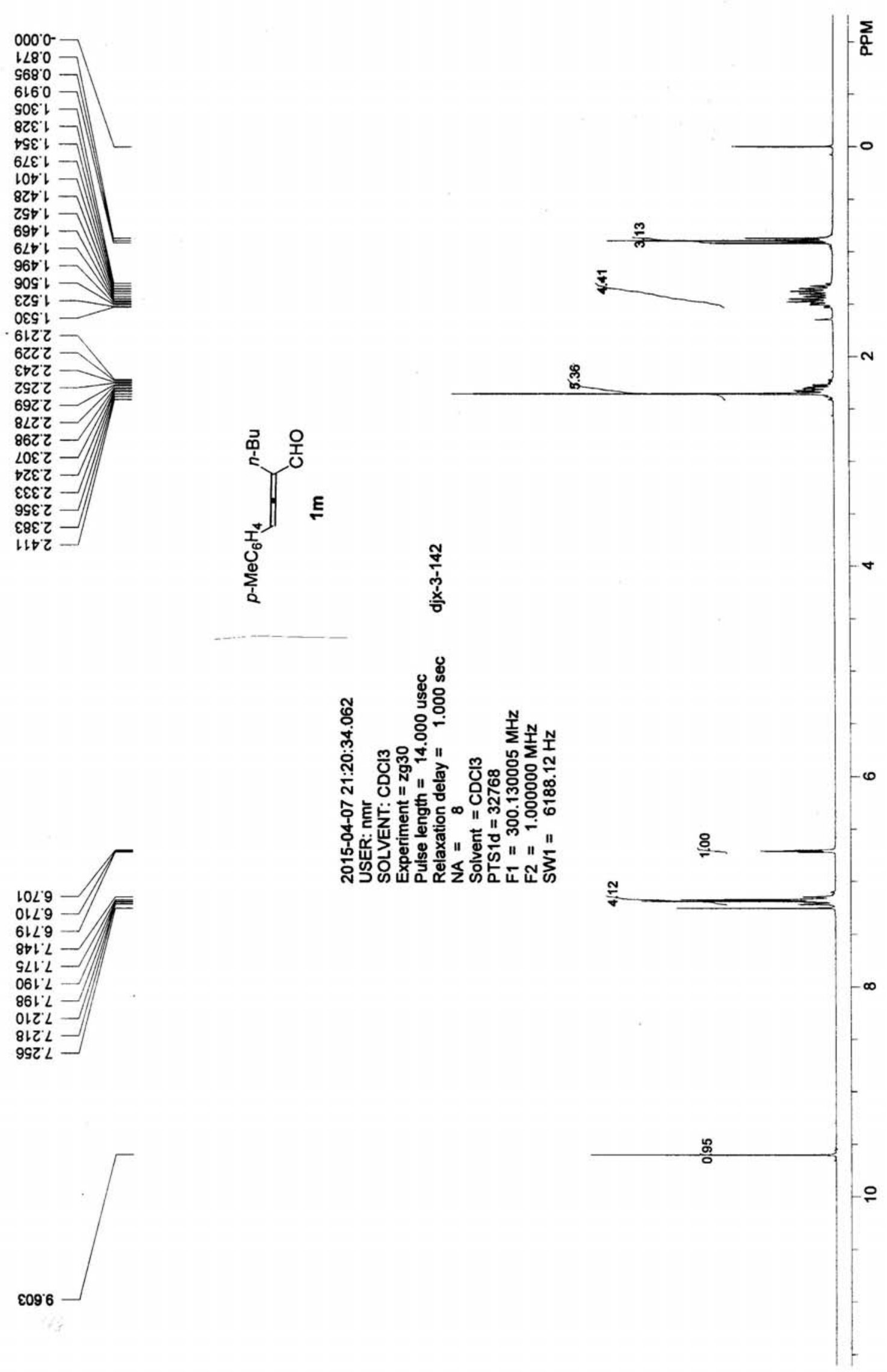

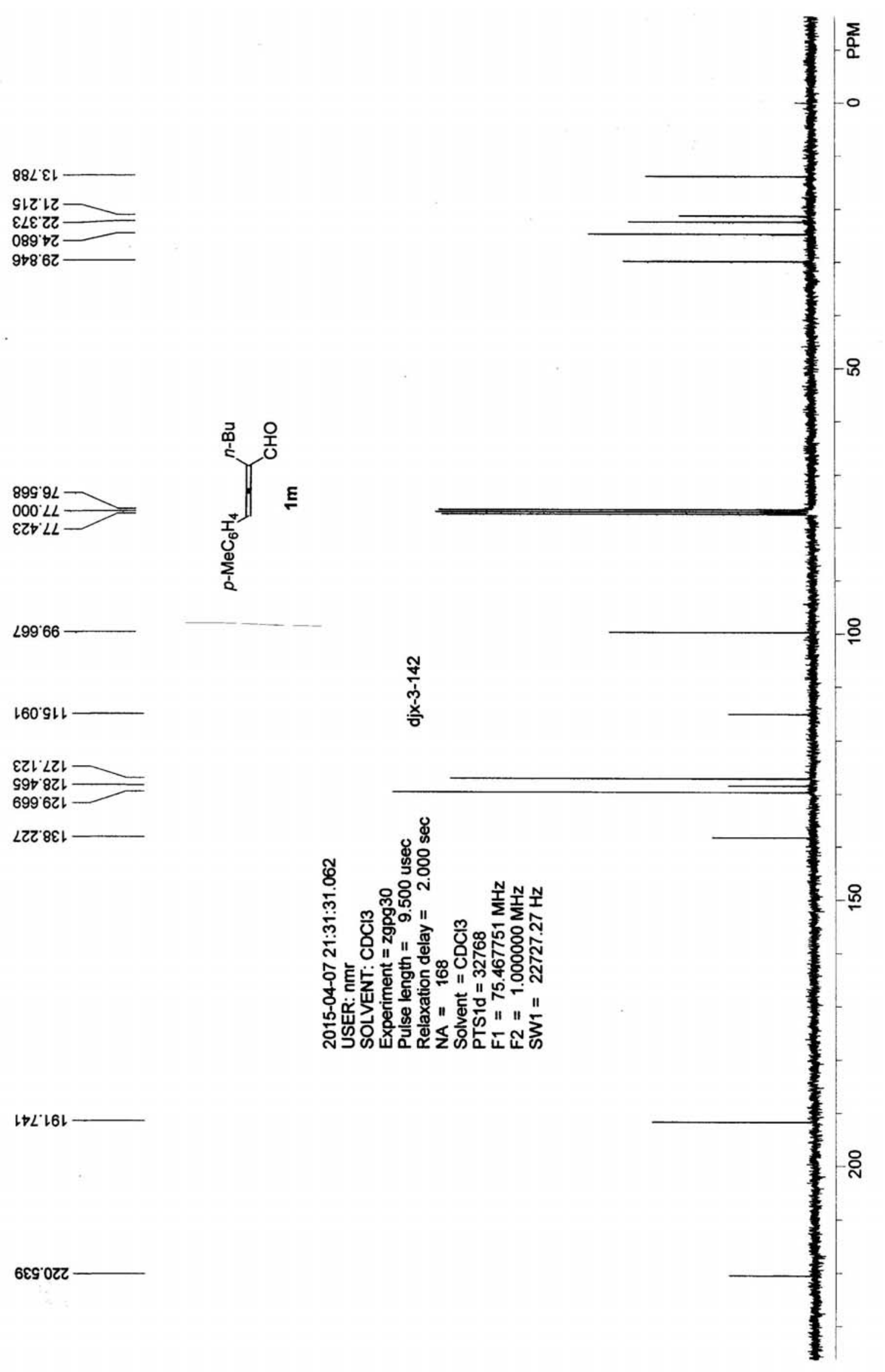

$6 \varepsilon 9.02 z$ 
$000^{\circ} 0$

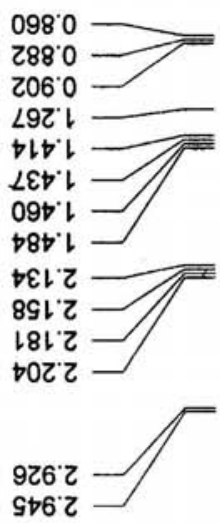

$120^{\circ} \mathrm{S}$

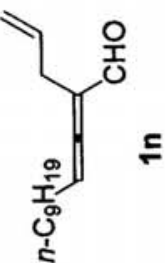

|| \begin{tabular}{l|l}
$\sum_{0}$ \\
0
\end{tabular}

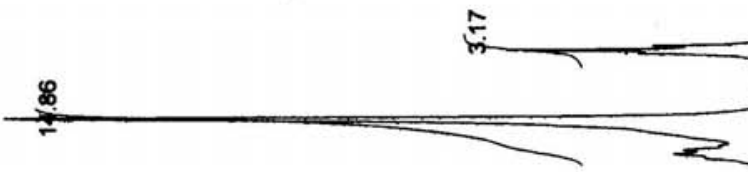

ஜ્ष

S9O'G

OEL' $\mathrm{G}$

$\angle \varepsilon L 97$

ZSL'S

$19 L \cdot 9$

$98 L ' S$

$608^{\circ} 9$

$0 Z 8$ '

¿ह8 9

$\varepsilon \succ 8$ '

S98. 9

$\varepsilon 8 Z^{\circ} L$
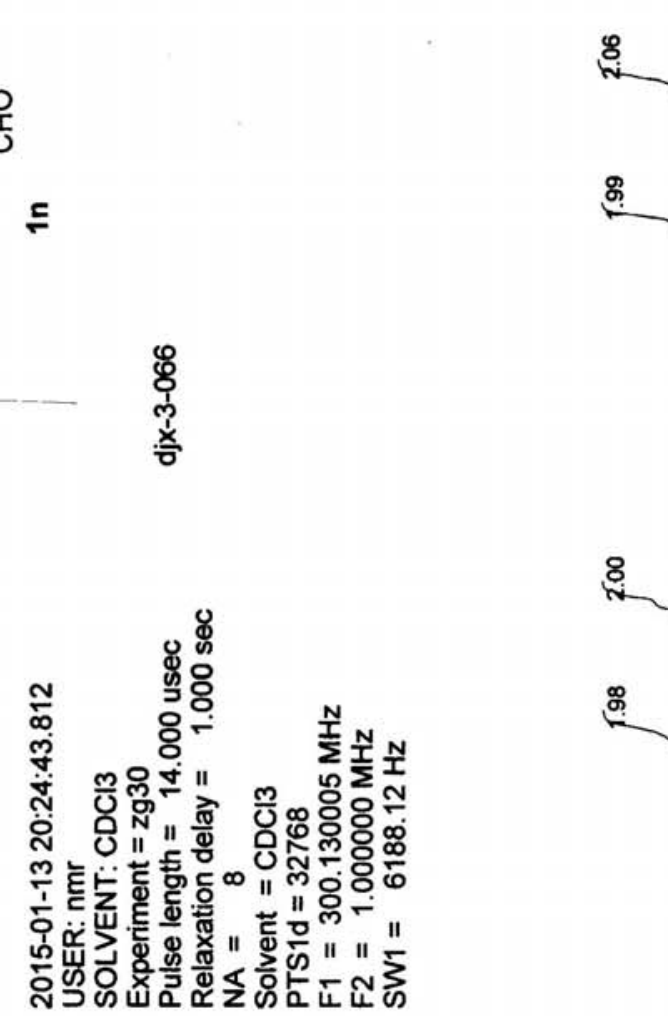

g

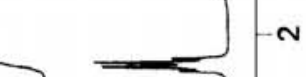

$\underset{\text { g }}{8}$

\%

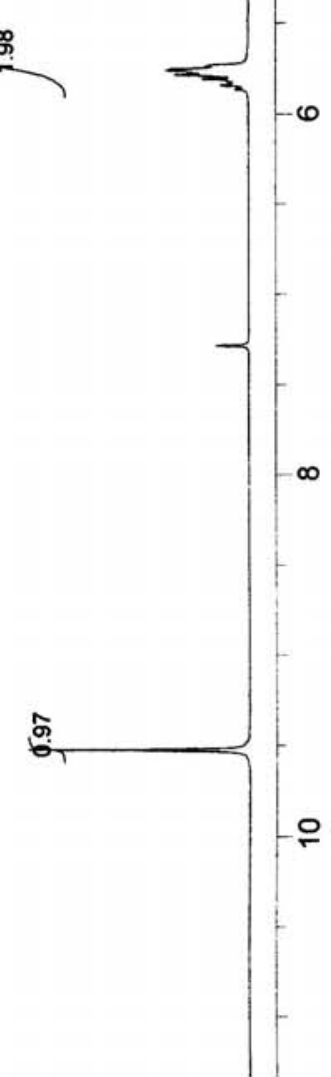



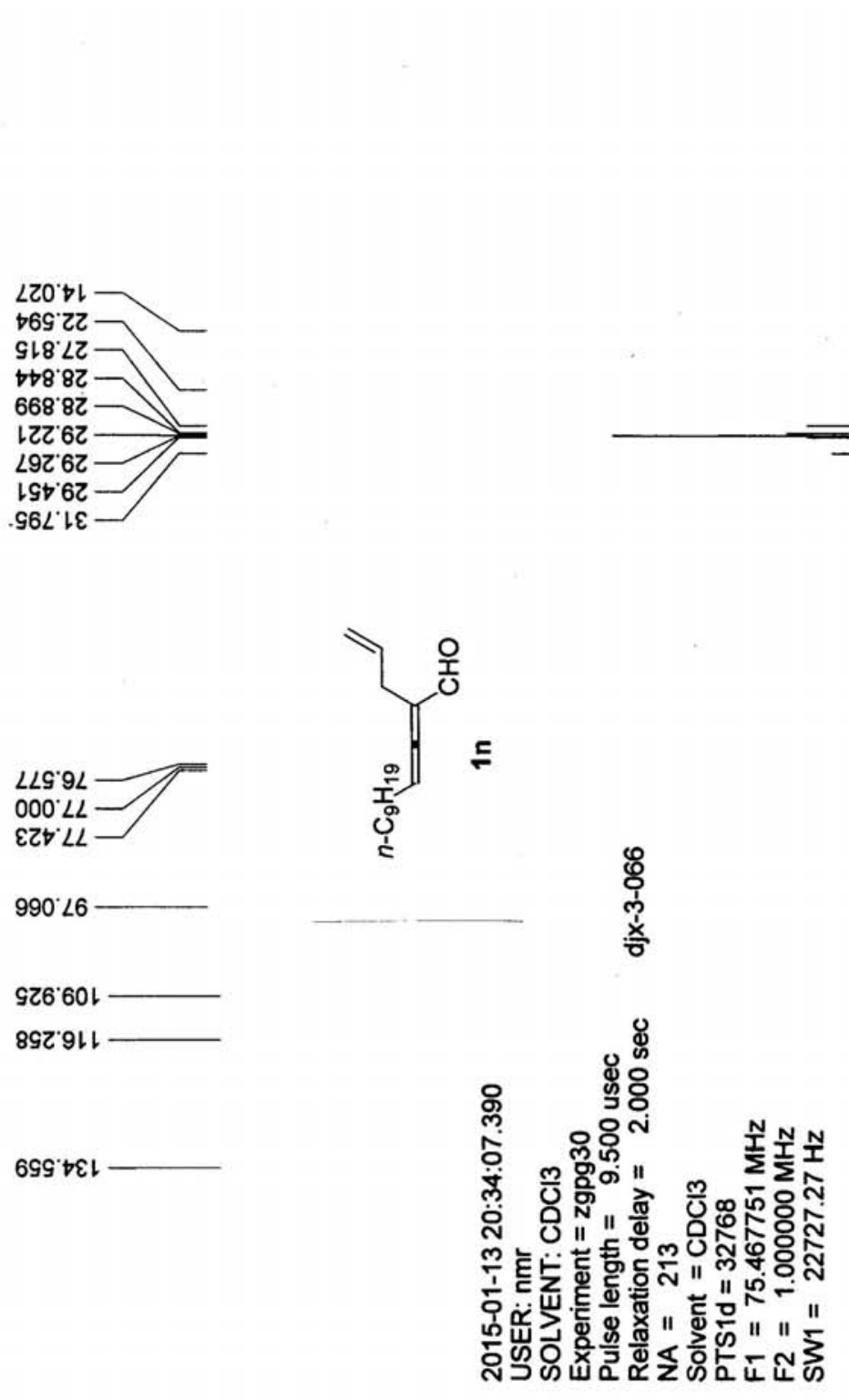

$699^{\circ} \sqcup \varepsilon\llcorner$

$\frac{5}{5}$

9Z6.60 -

89ट 9เ

9๕o' $26 \downarrow$

$\angle 0 T 812$

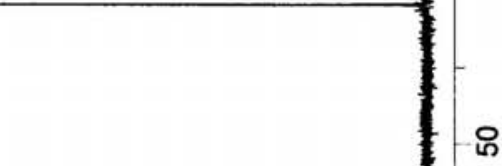




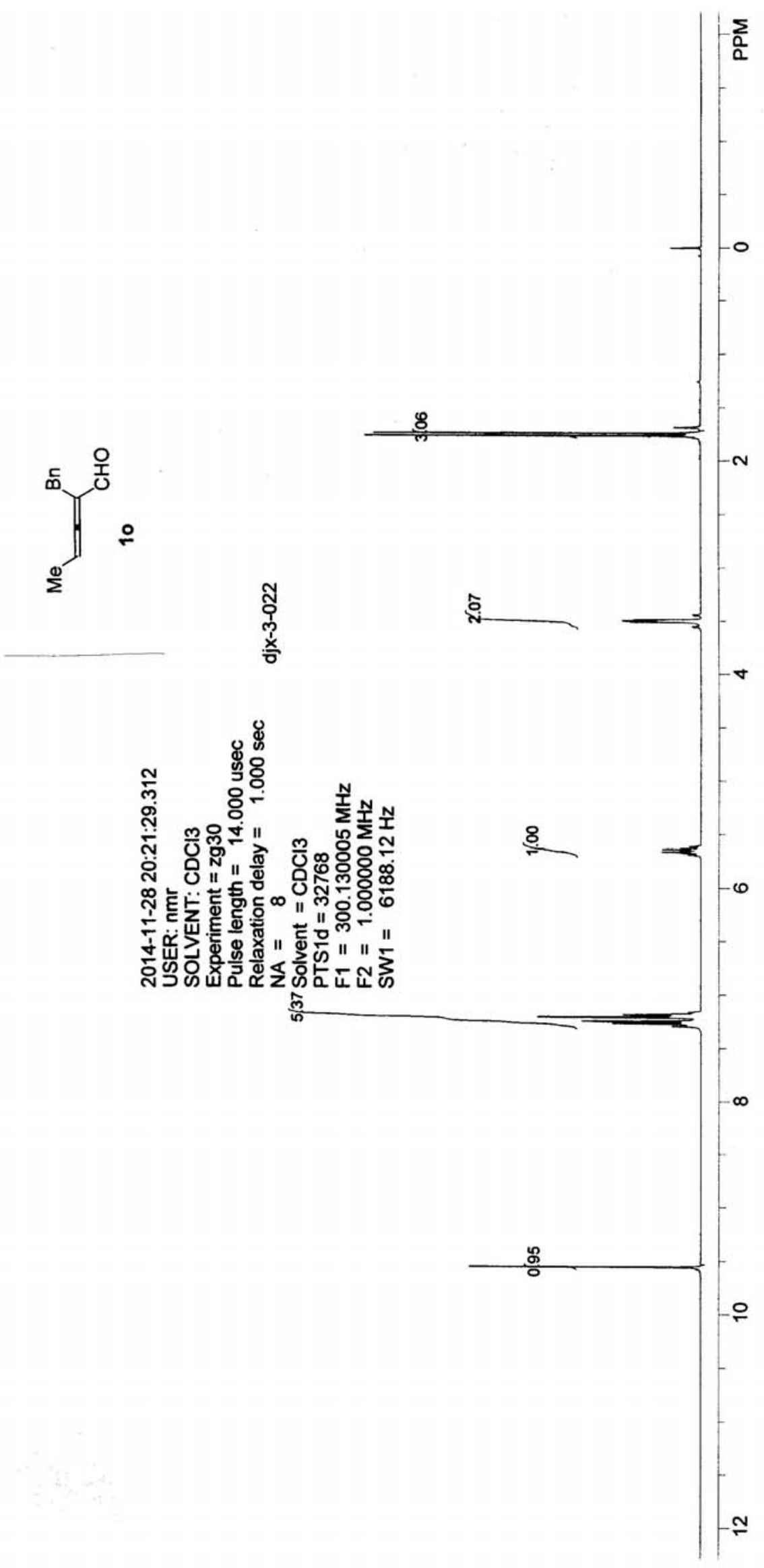


$886^{\circ} \mathrm{ZL}$

$\downarrow \varepsilon て \cdot เ$
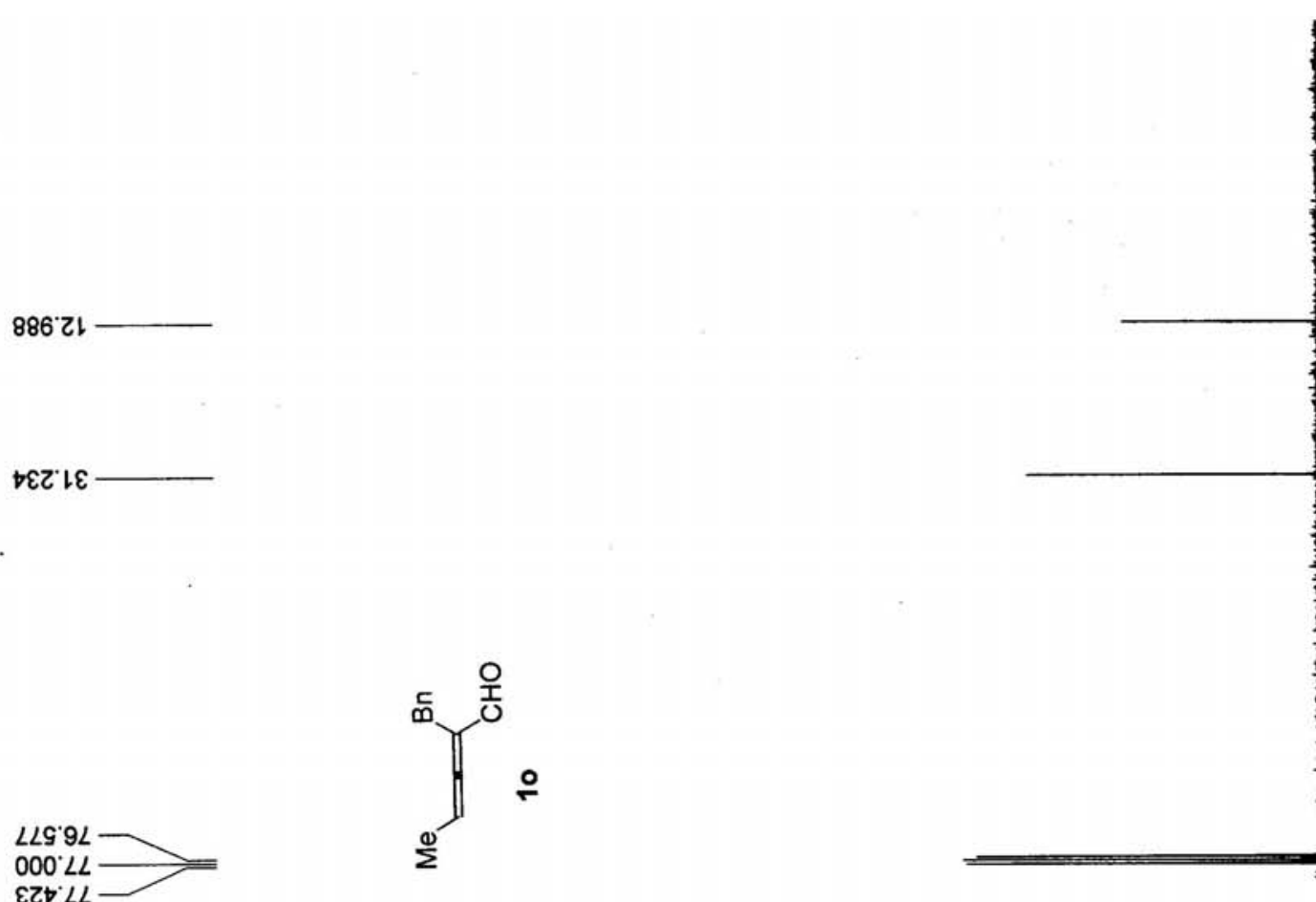

9ะ8 เ6

$86 L^{\circ}$ ㄴㄴ

సิ

†0Z 9 Z -

$\forall \forall Z$ ' $8 Z 1$

OSL $8 Z 1$

งZ6 8ะเ

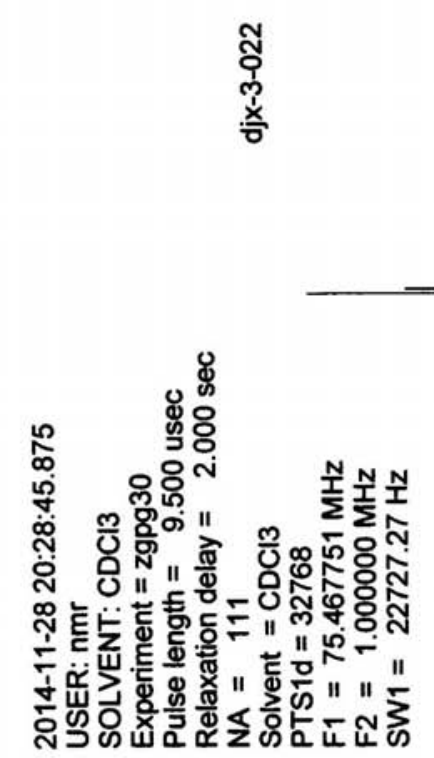

$\angle 06^{\circ} L 6$

682612

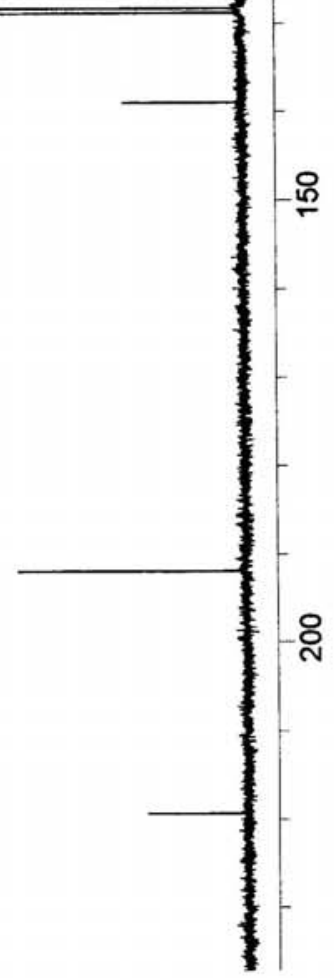



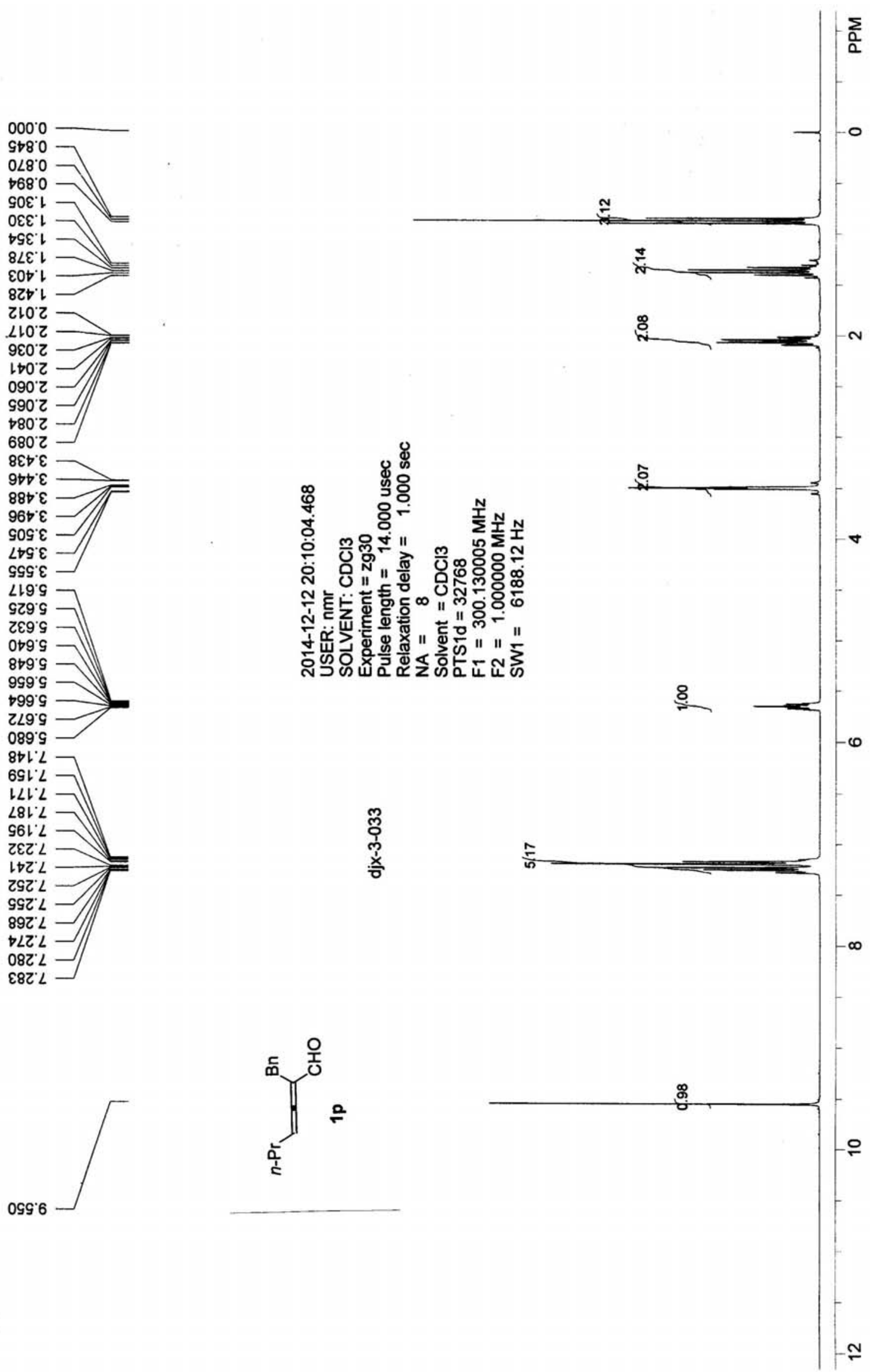


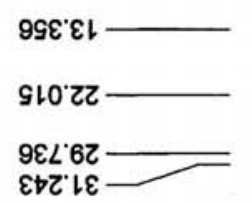

LLG' $9 L$

$000 \% L$

ZE† LL

$168^{\circ} 96$

89ยเレレー

6ع।'9ZL -

081.821

$\angle 8 L ' B Z L$

เ98. $8 \varepsilon 1$

$198^{\circ} 161$

$8 \varepsilon L^{\prime} 812$

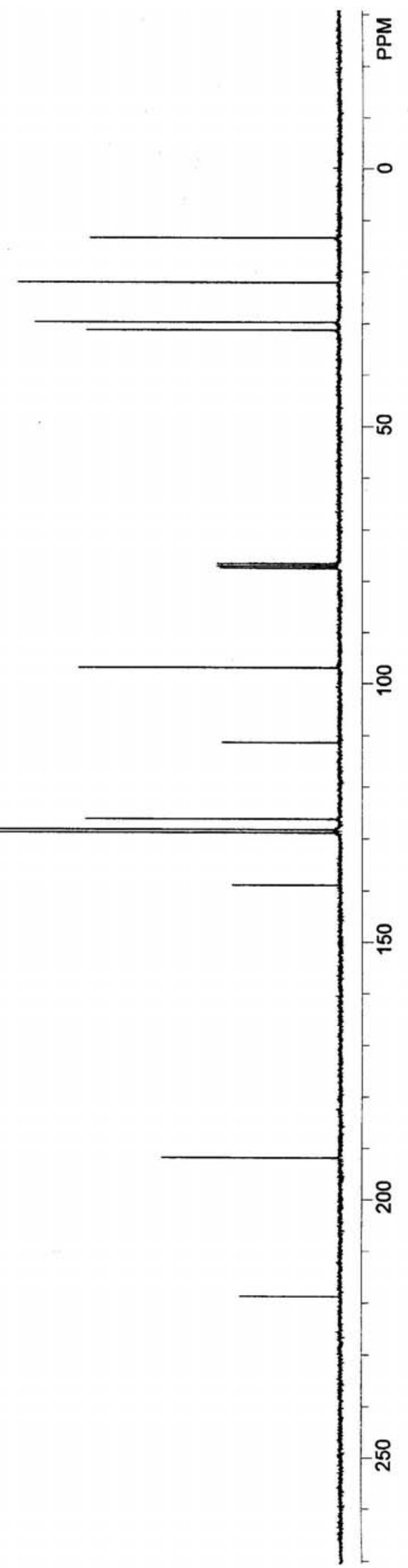



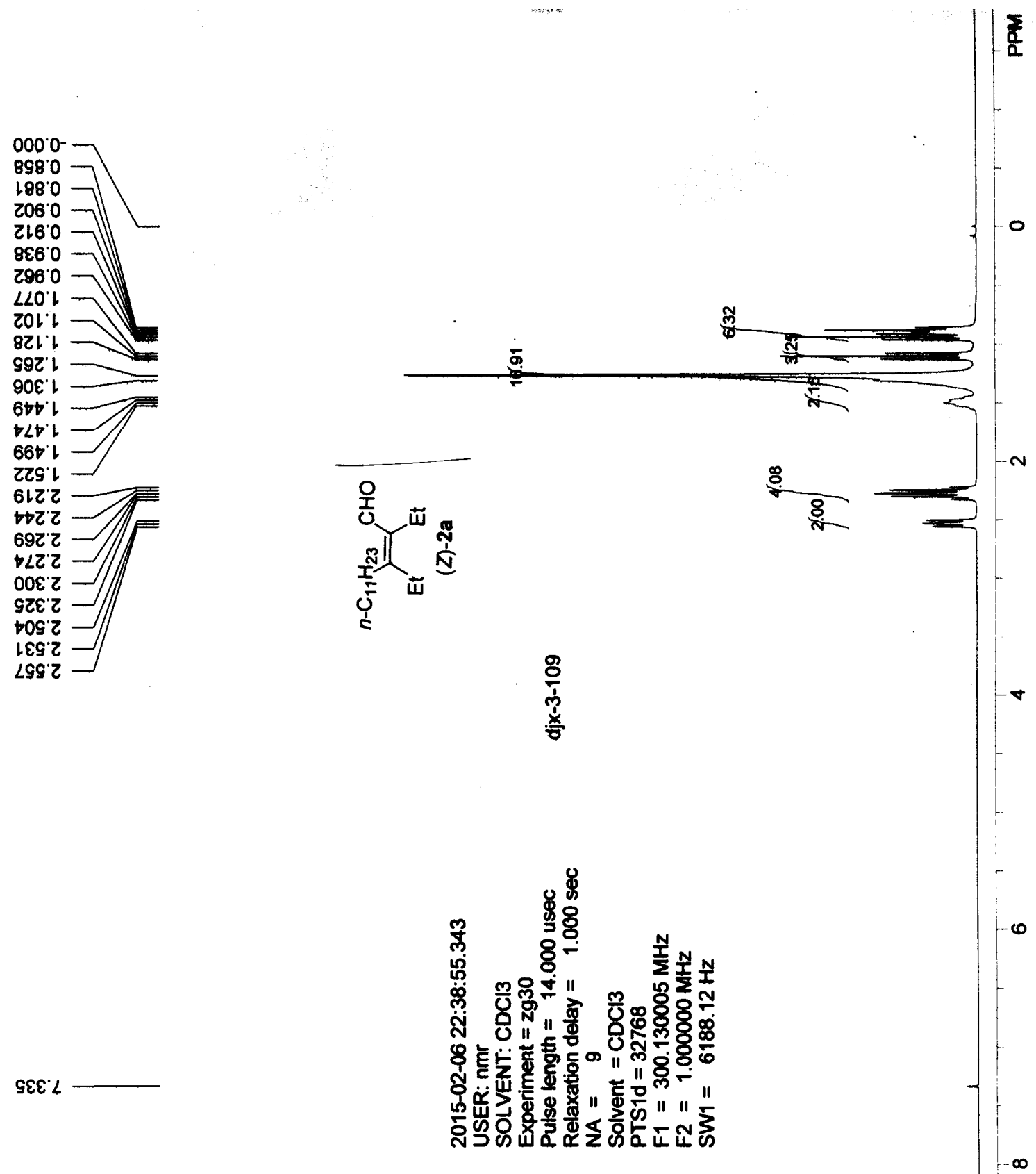

ธะ६. L ণิ宀

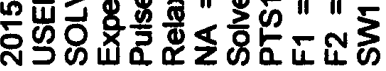

$9900 \mathrm{O}$

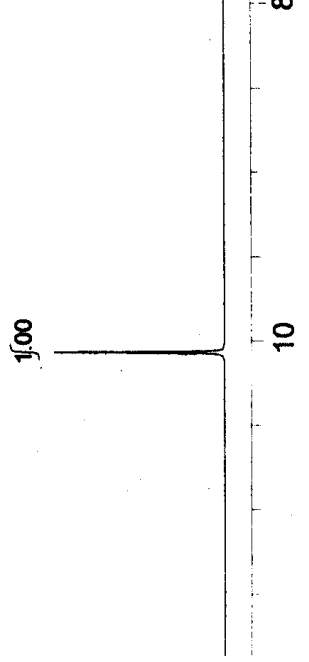




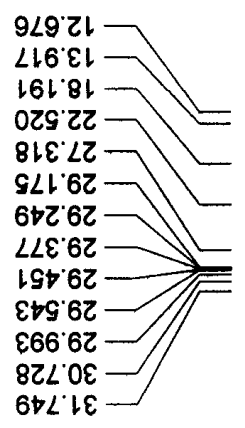

$\mid$ t宫
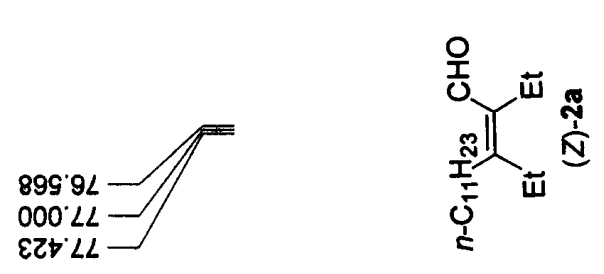

$\frac{8}{0}$
के
xx

$\mid Z L ' \angle E L$

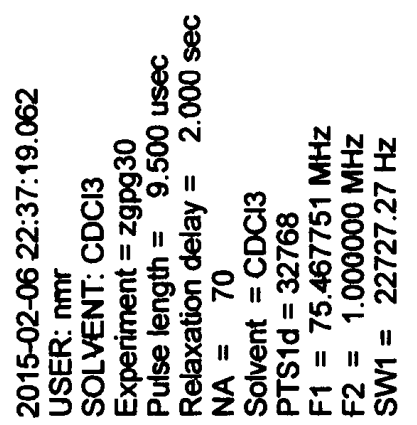

Lا'६9।

$+t 1 \cdot 61$

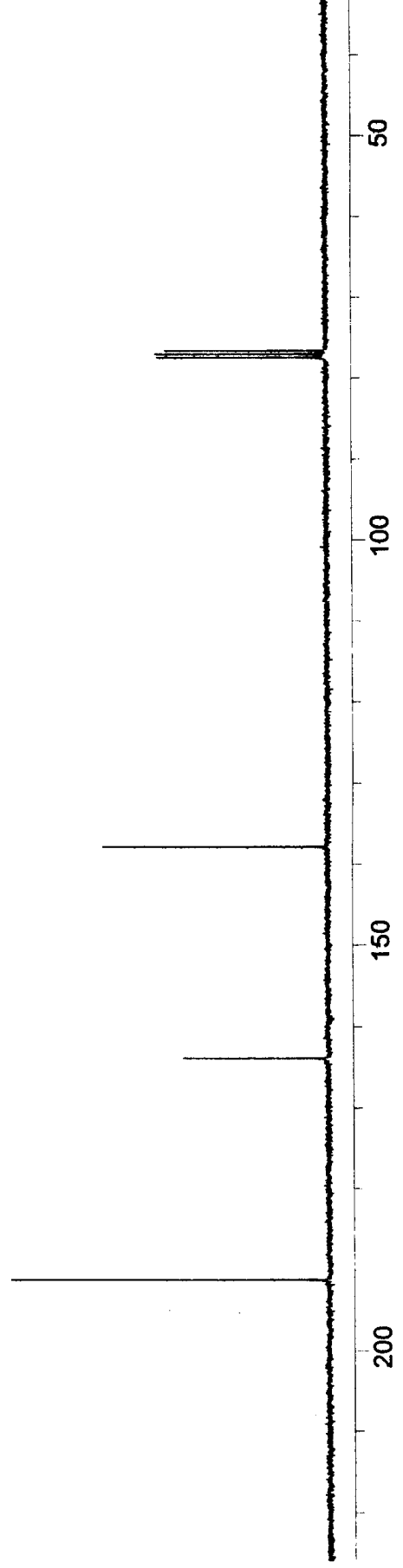




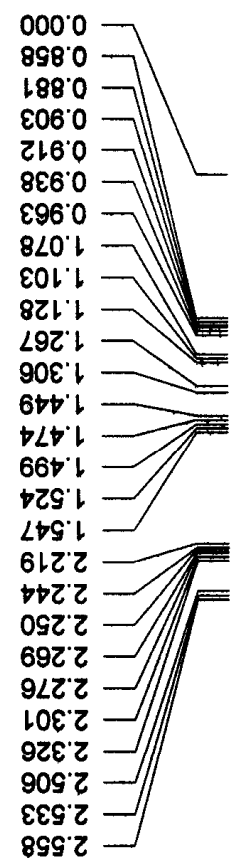

$\llbracket\left[\begin{array}{c}\frac{5}{0} \\ 0\end{array}\right.$

859

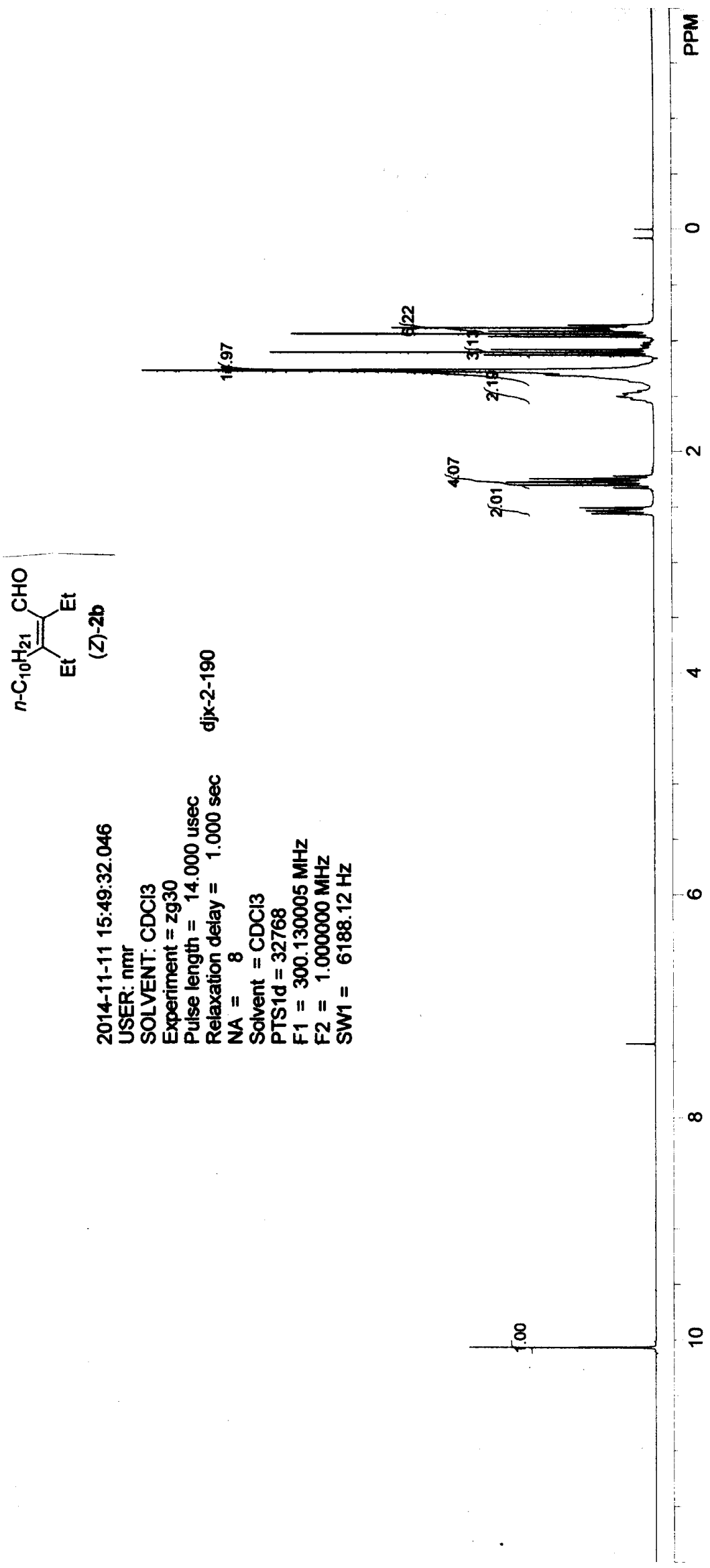



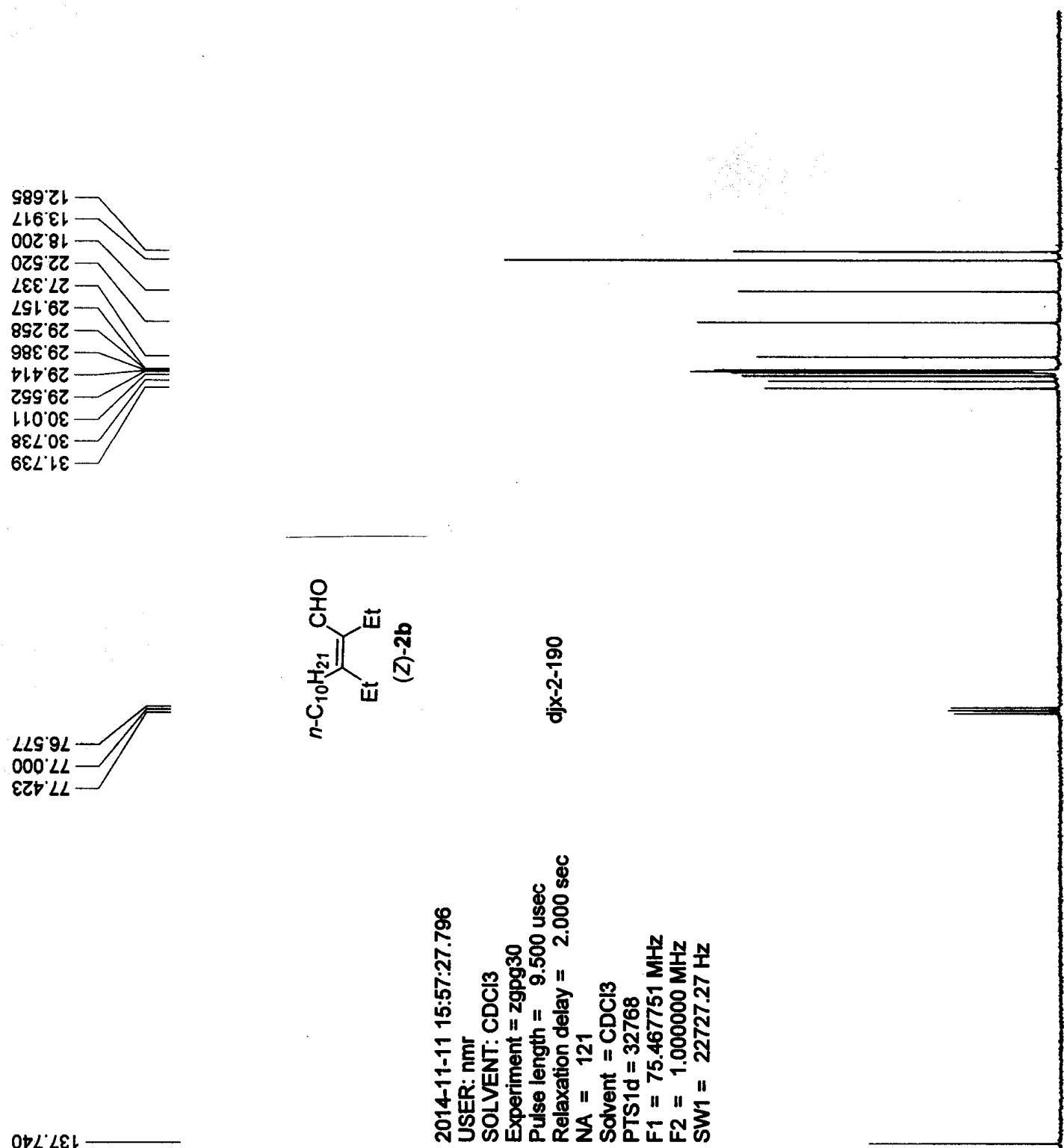

$668^{\prime} \varepsilon 9$

$66 ! \cdot 6 l$

$\frac{8}{\frac{\grave{1}}{x}}$ 

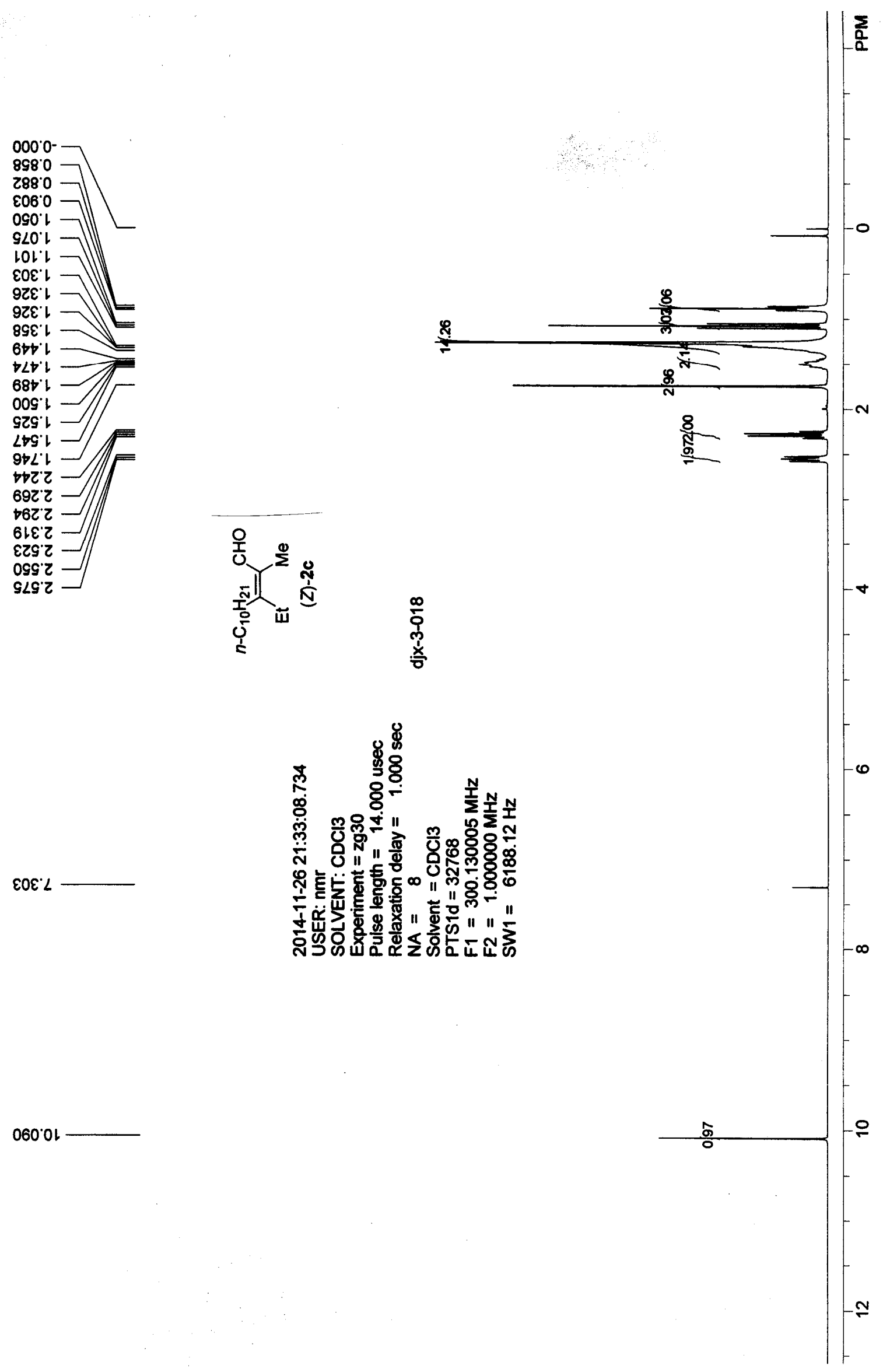

$060^{\circ} \mathrm{OL}$

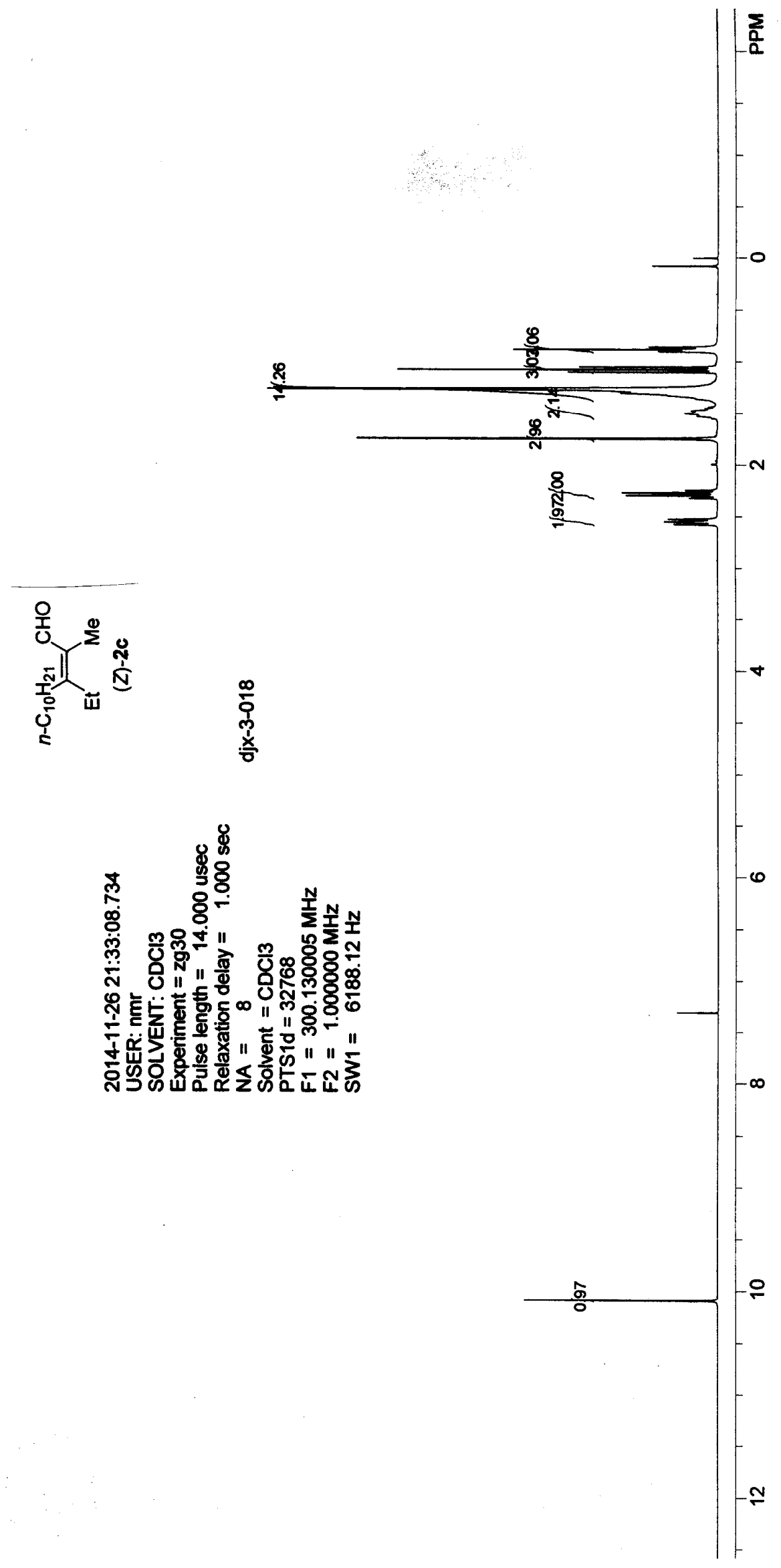

$\varepsilon 0 \varepsilon$ L 

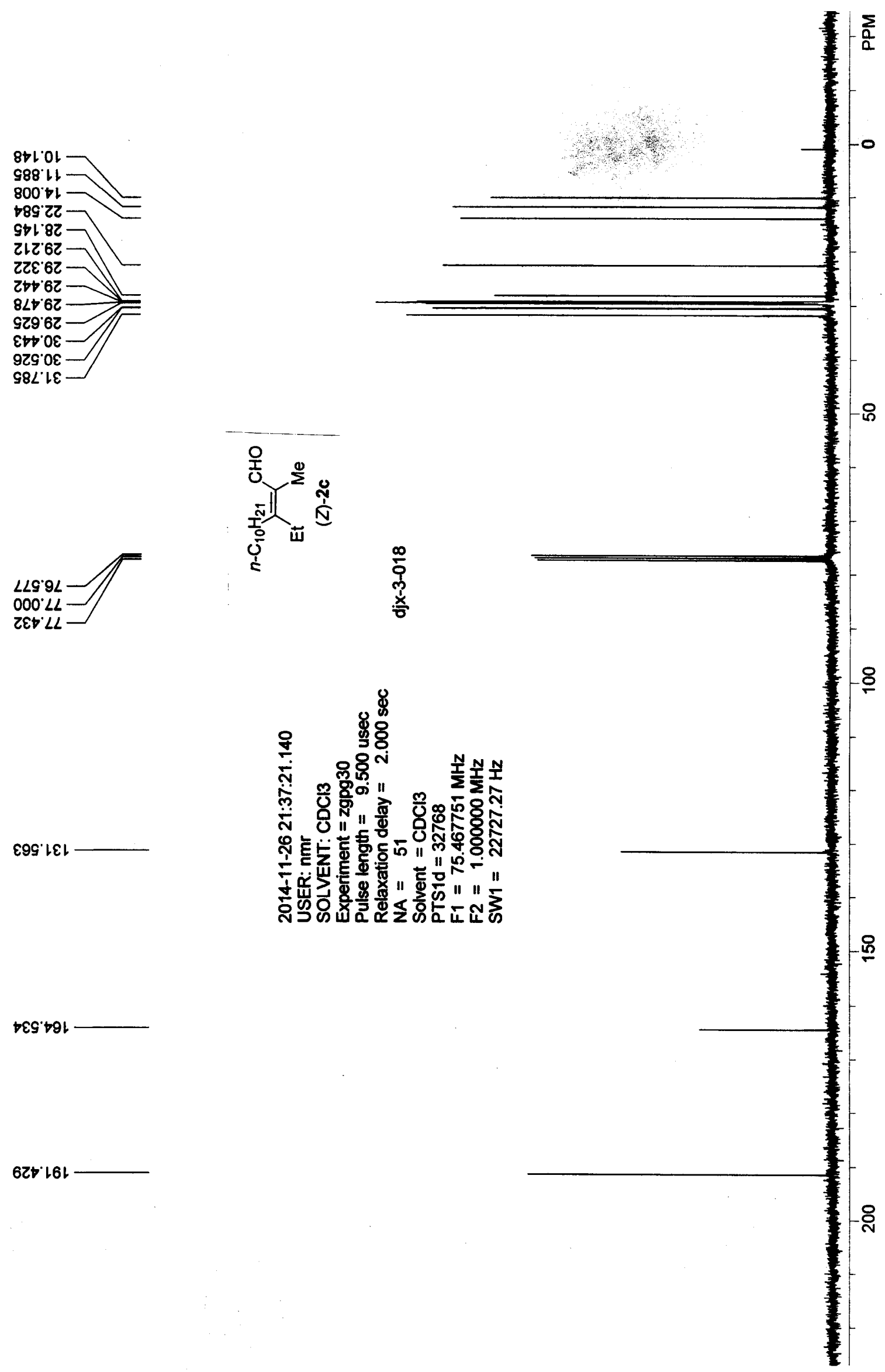

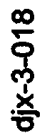

ع9s'เยเ

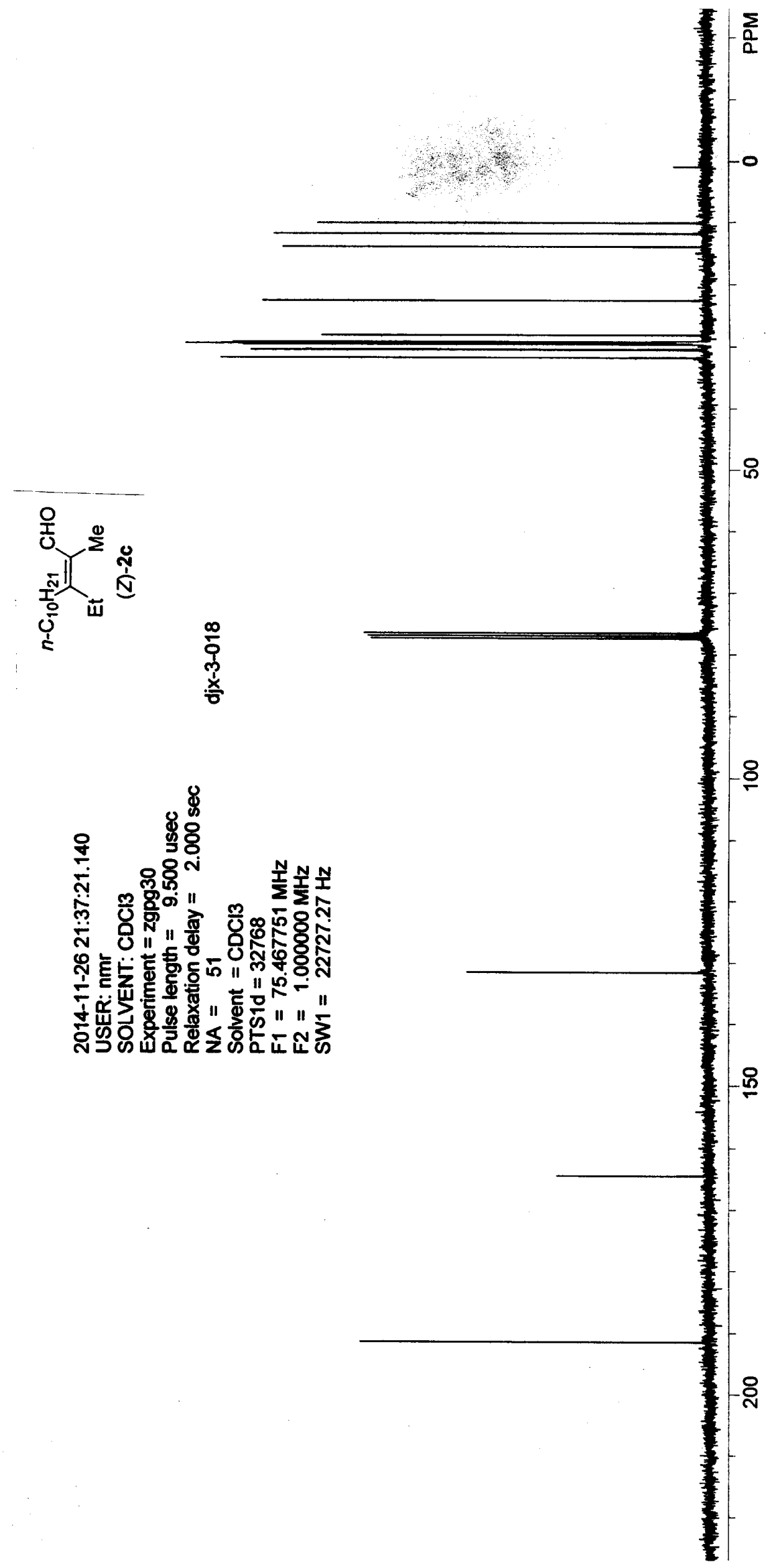

$\downarrow \varepsilon S^{\prime} \triangleright 9 \downarrow$

$62+161$ 

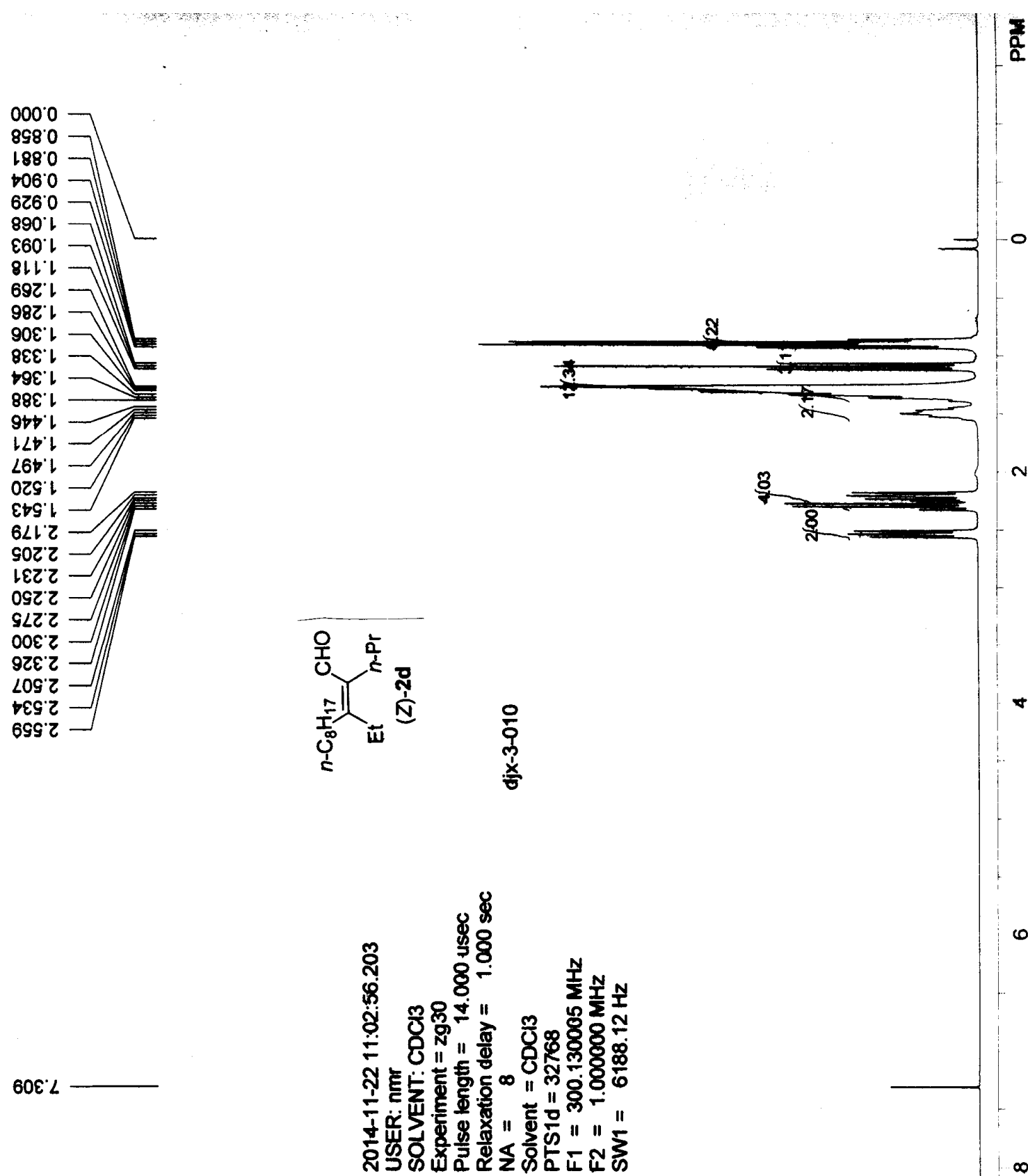

$60 \varepsilon \%$

$1 \angle O^{\circ} O L$

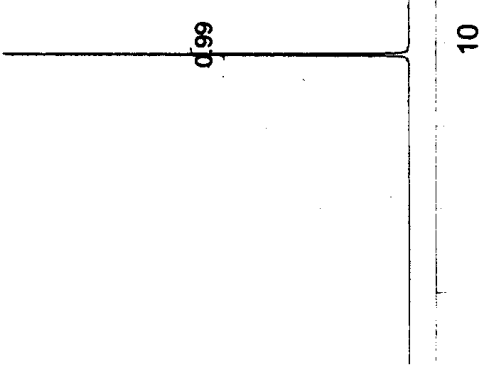



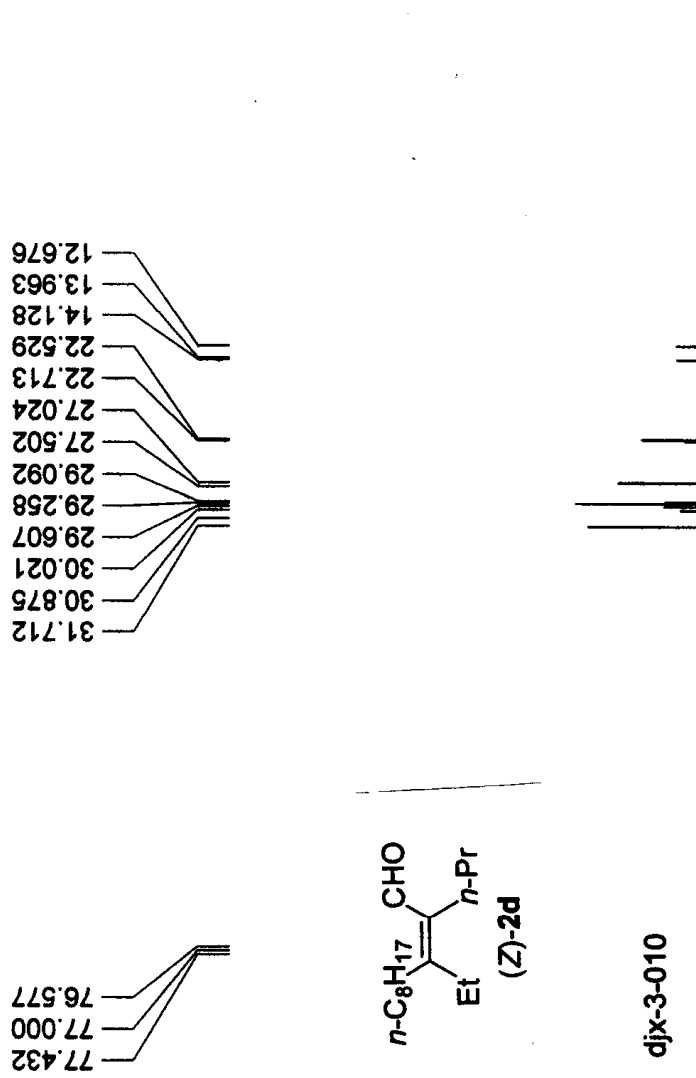

$\angle 8 Z 9 \varepsilon$

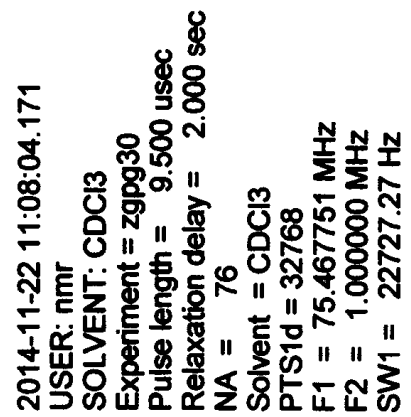

$69 b \vdash 91$

8S9' L6L 

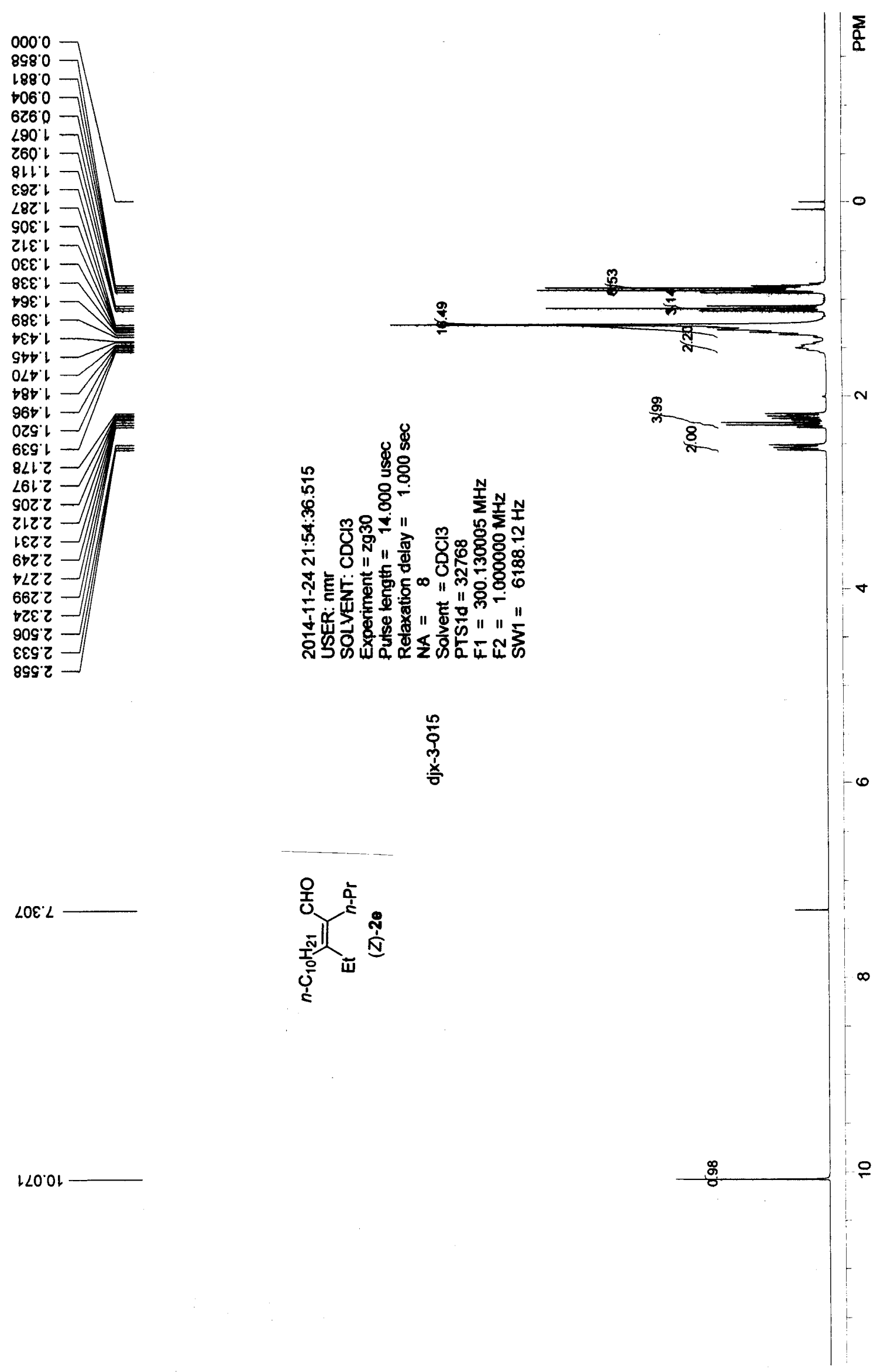

$1 \angle 0^{\circ} O \mathrm{~L}$ 

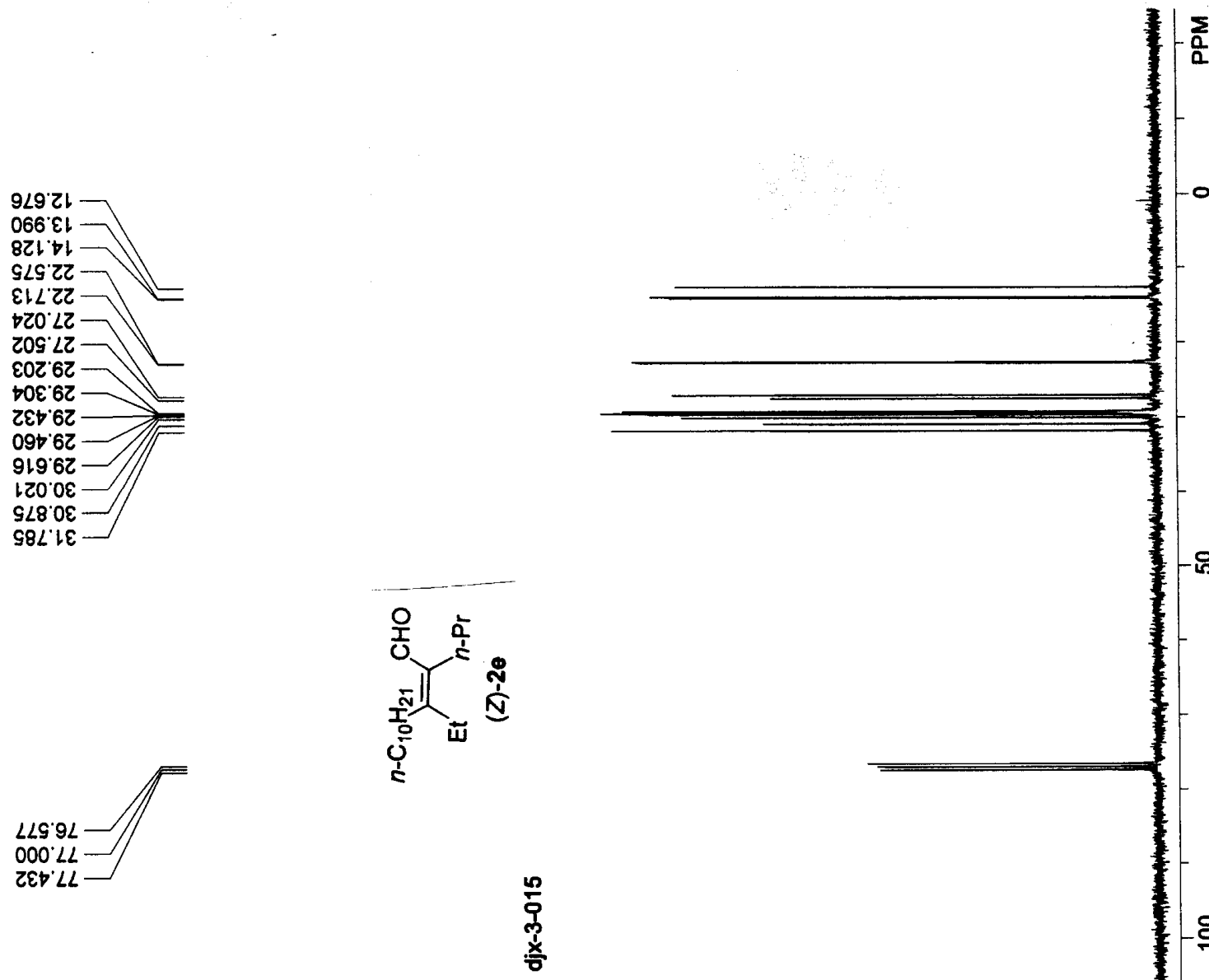

L8Z'9\&L

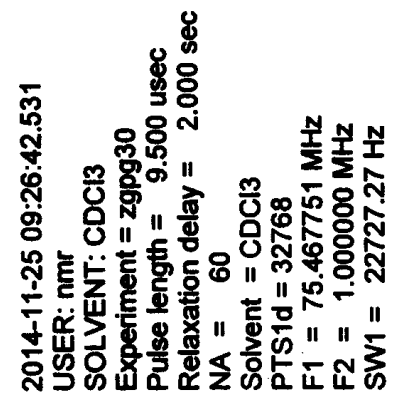

8 ' $16 t$ 

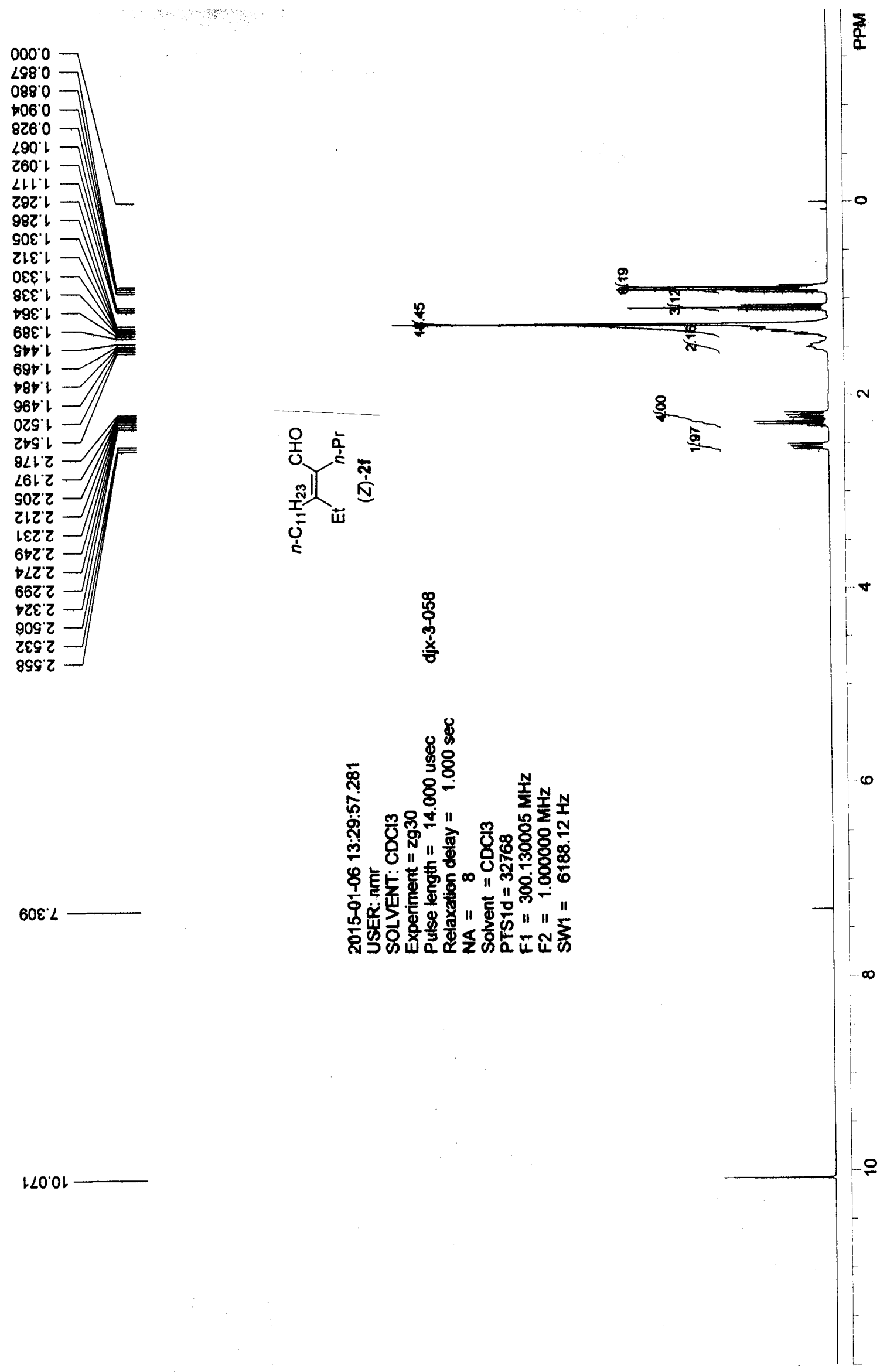

$60 \varepsilon^{\circ} L$

$1 \angle 0 \circ O$

오 


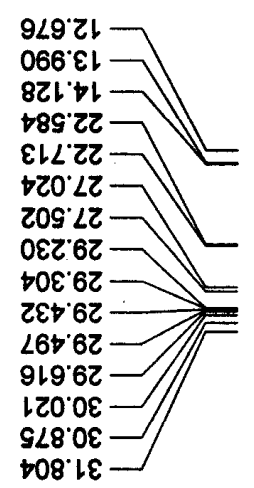

$\mid+2$

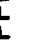

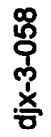

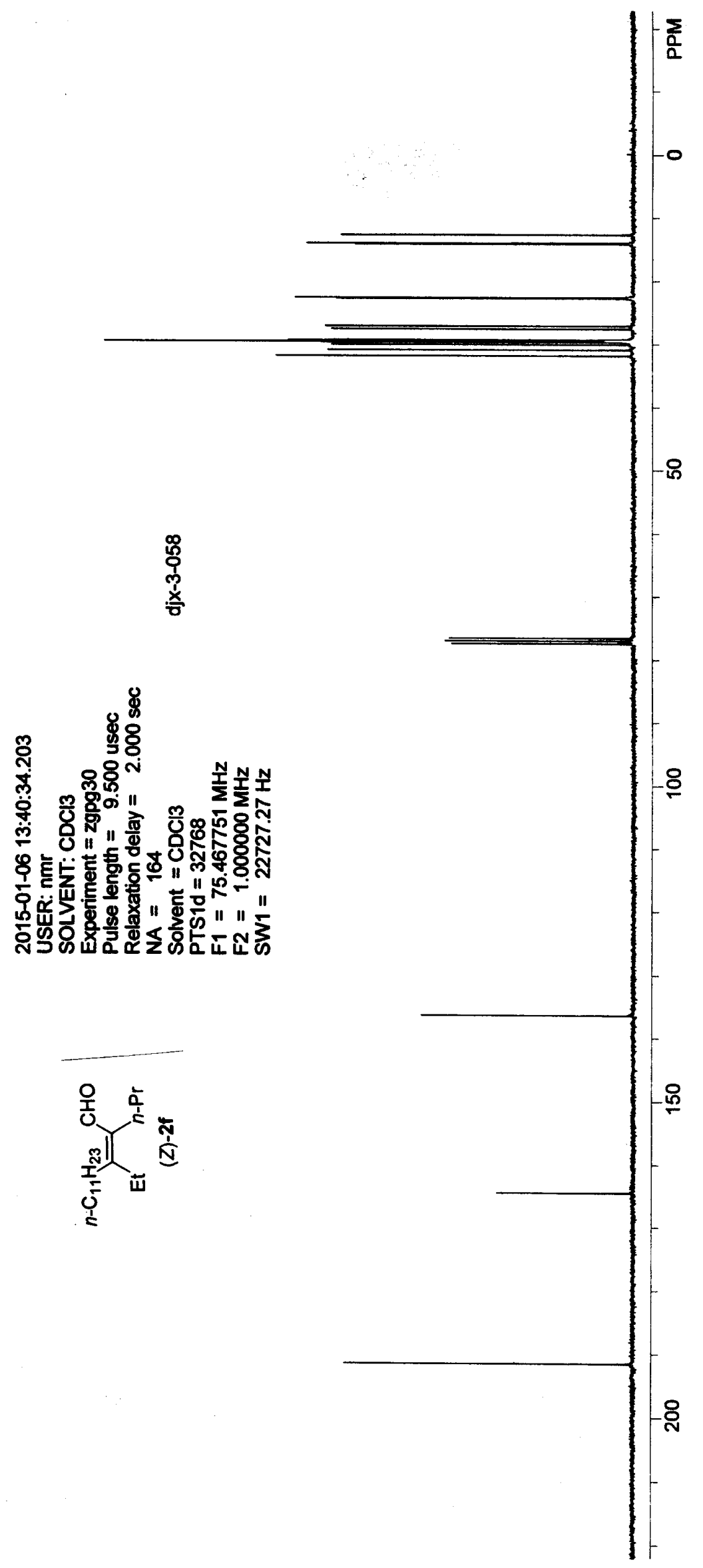

$\angle 8 Z^{\circ} 9 \varepsilon$

$000.2 L$

$\varepsilon ट \downarrow \backslash L$

$\left|Z 9^{\prime}\right| 6 \mid$ 

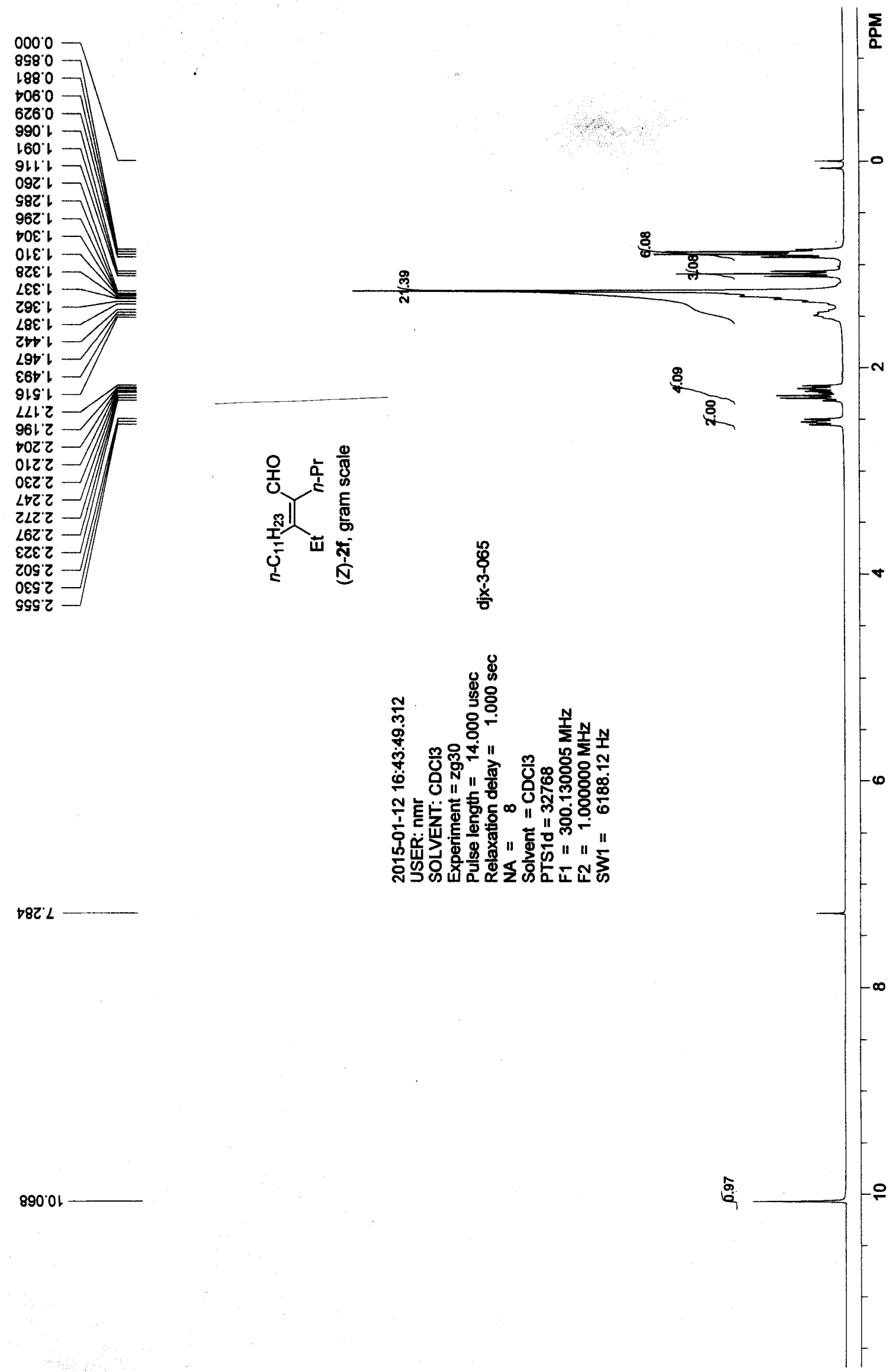

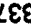

ट98

$\angle 90^{\circ} l$

E6เ'

olc

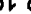

o\&Z

$\angle \forall Z Z$

ZLZ

sGs'

$890^{\circ}$ O

$\nabla 8 Z^{\circ} L$
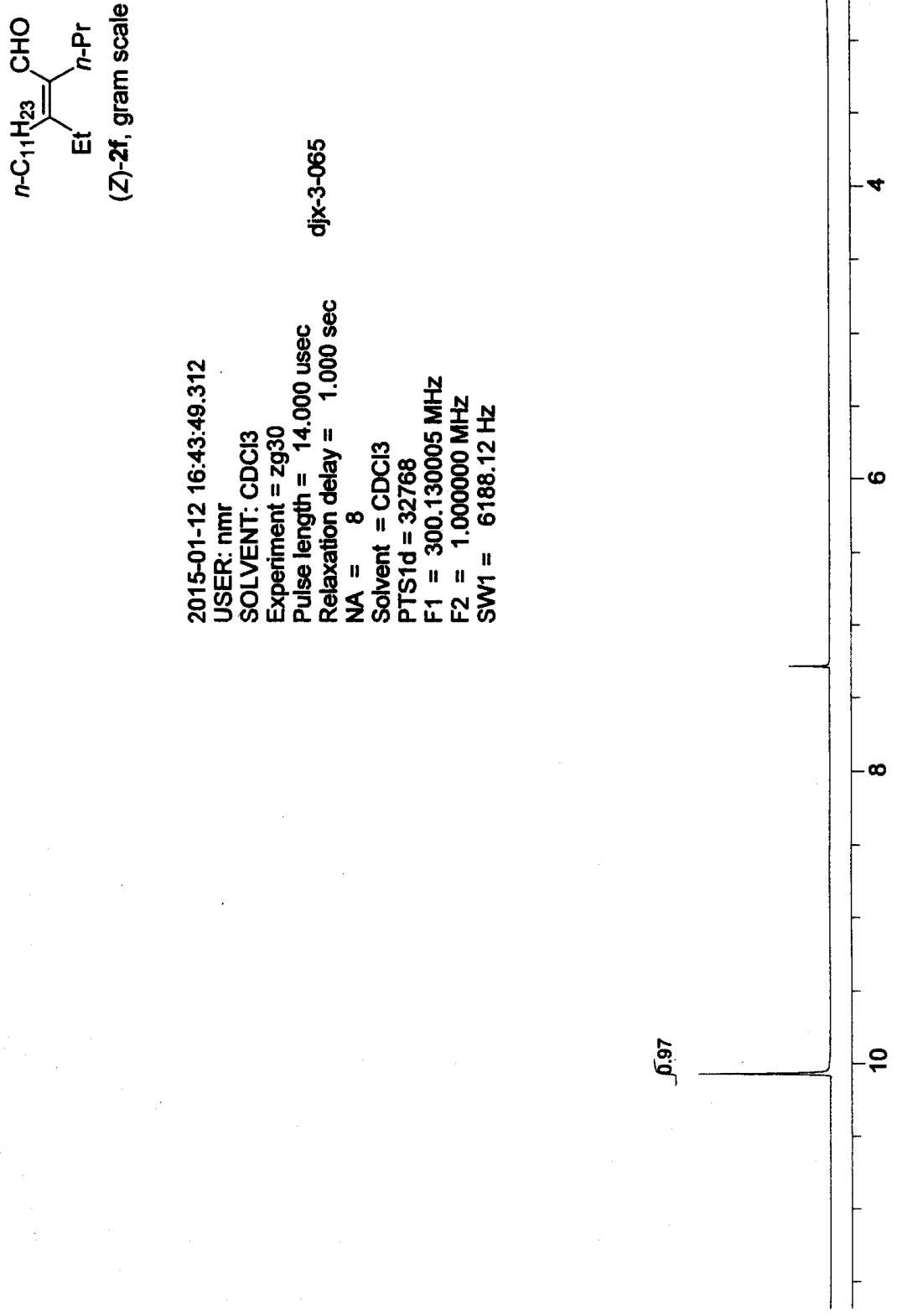

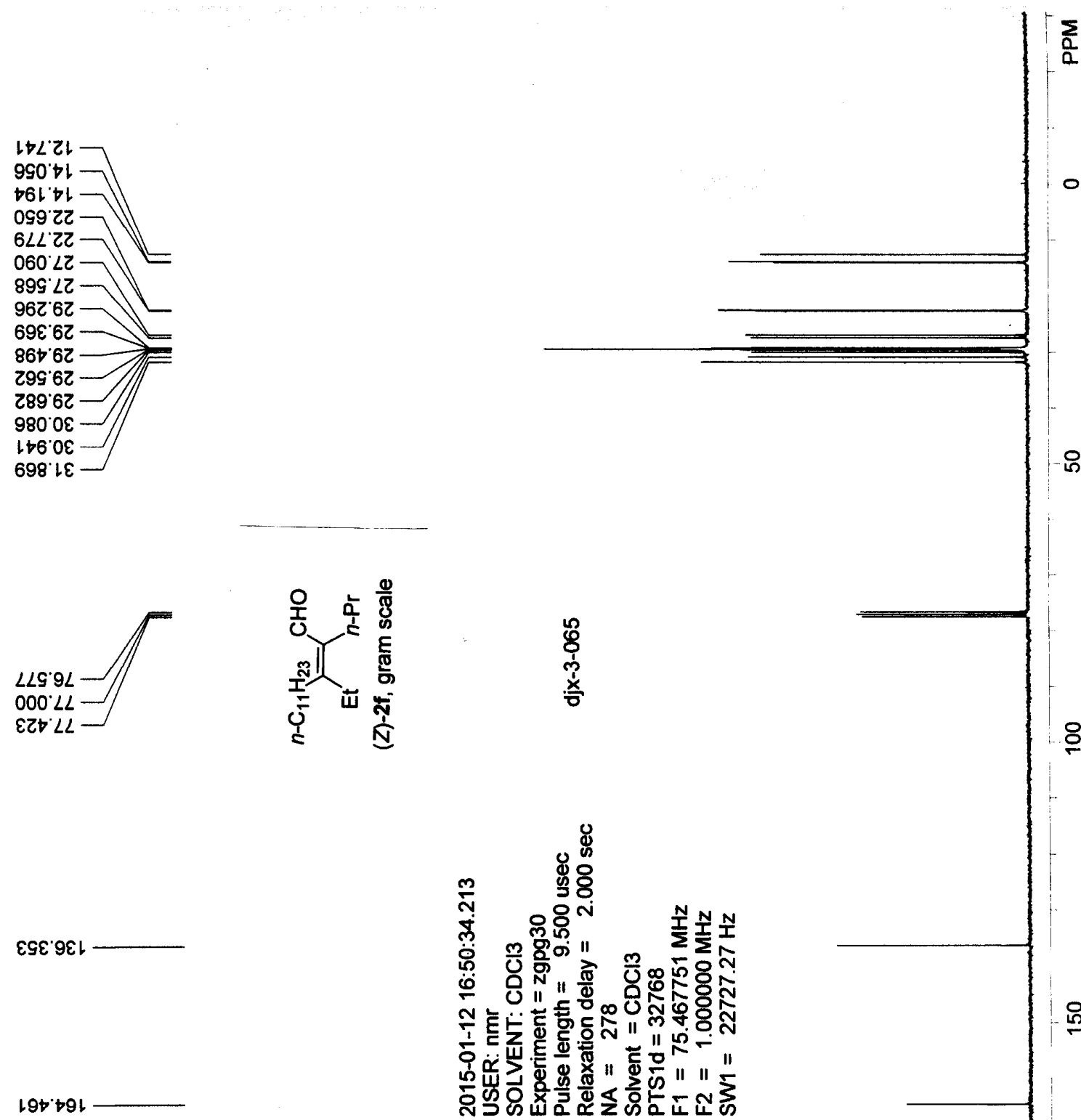

$989^{\prime} L 6$

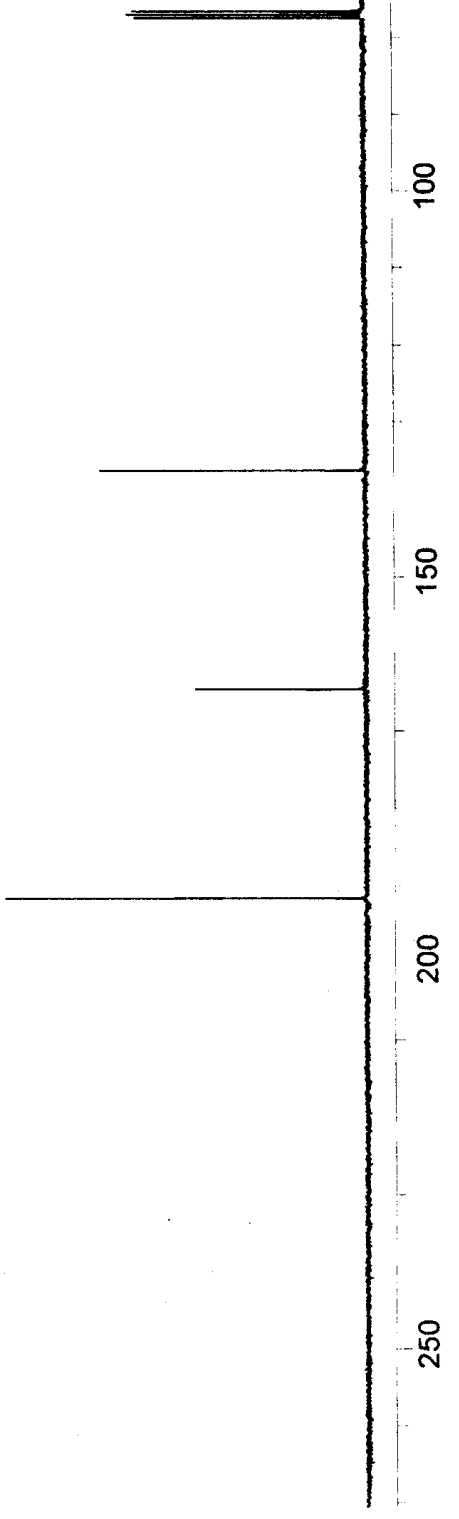



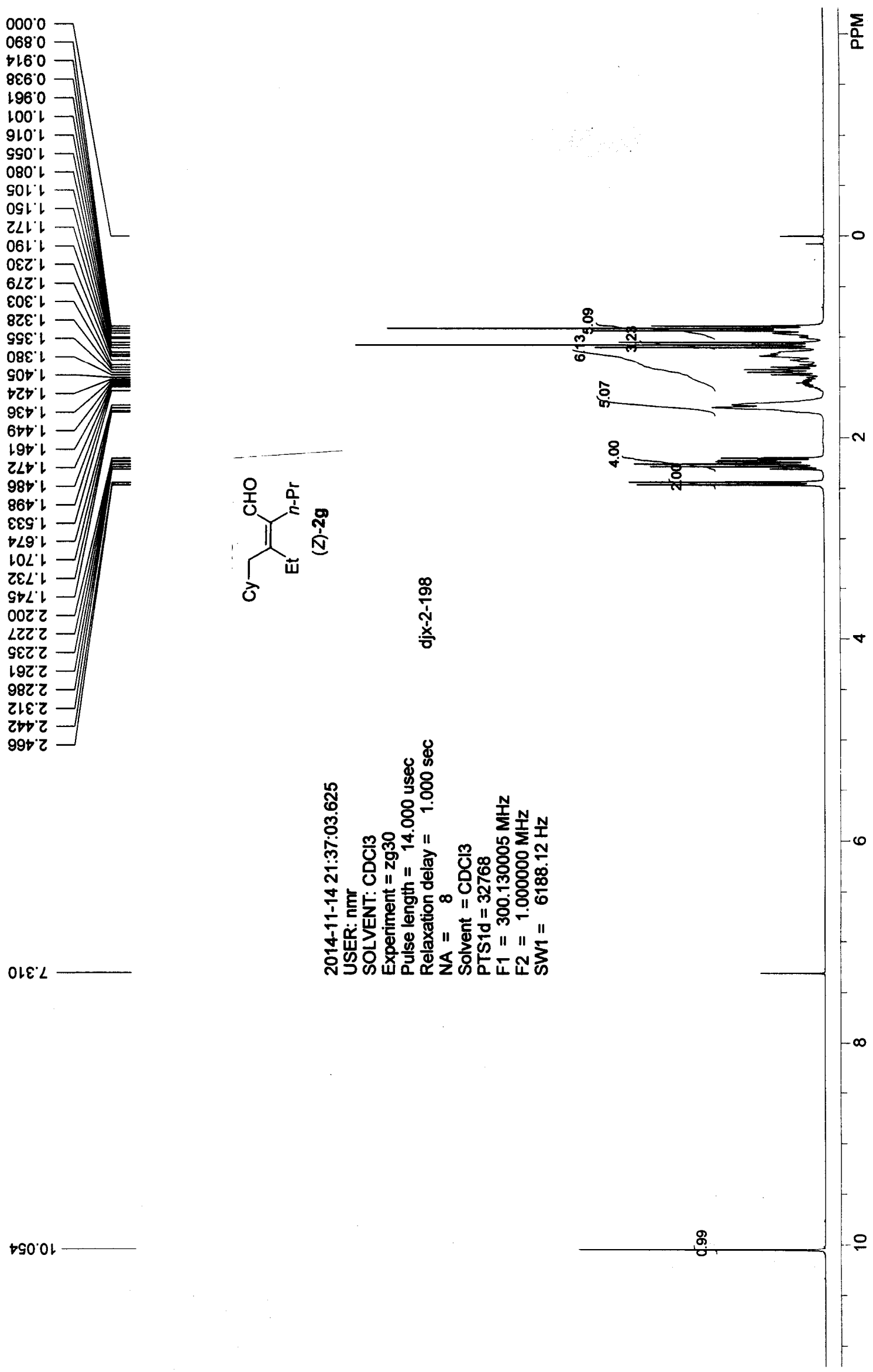
$08 \mathrm{~S} 2$

$090 \circ 1$

169 22

เᄂL 92

EHLLZ

$80 \mathcal{S}^{\prime} \angle Z$

เดเ ह

เ 16.98

ZSเ' $8 \varepsilon$

ع89' $9 L$

$900 \angle L$

$8 \varepsilon+\angle L$

$68 S^{\circ} \angle \varepsilon$

E99' 291

$\left\llcorner 9 \varepsilon^{\prime}\llcorner 6 \downarrow\right.$

$\mid$ t亯

$\frac{1}{2}$

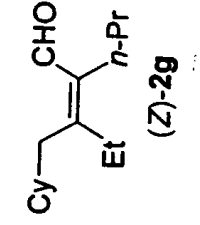

$\frac{\mathscr{Q}}{\stackrel{\%}{\grave{1}}}$

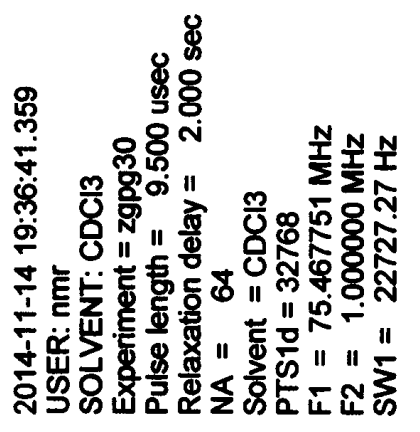

$\stackrel{8}{\circ}$

$\stackrel{8}{\circ}$

웅 

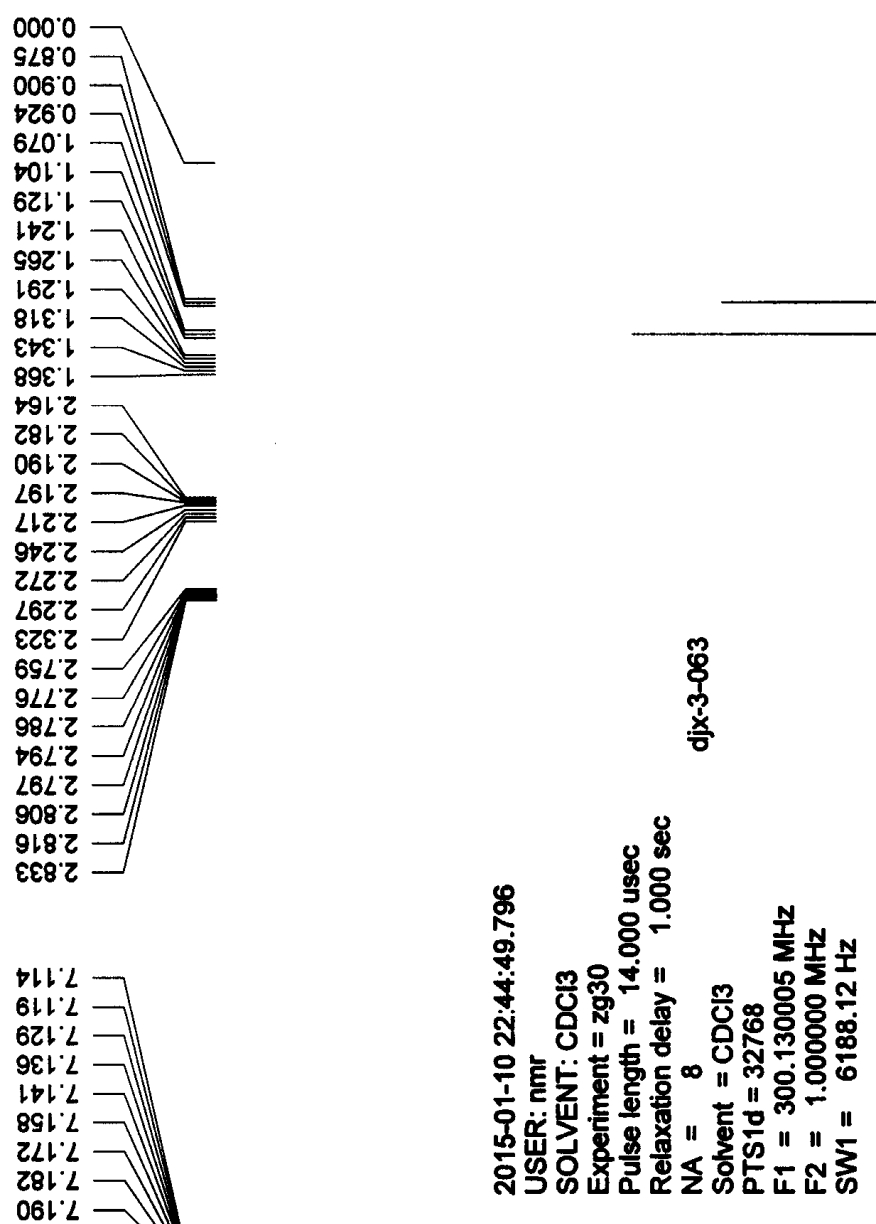

loz'

902 '

। $2 L$

\&\&Z

ItZ'L

$S t Z ' L$

$09 z<$

S9Z'L

$\backslash L Z L$

$282 \mathrm{~L}$

$882 \mathrm{~L}$

E6Z' 2

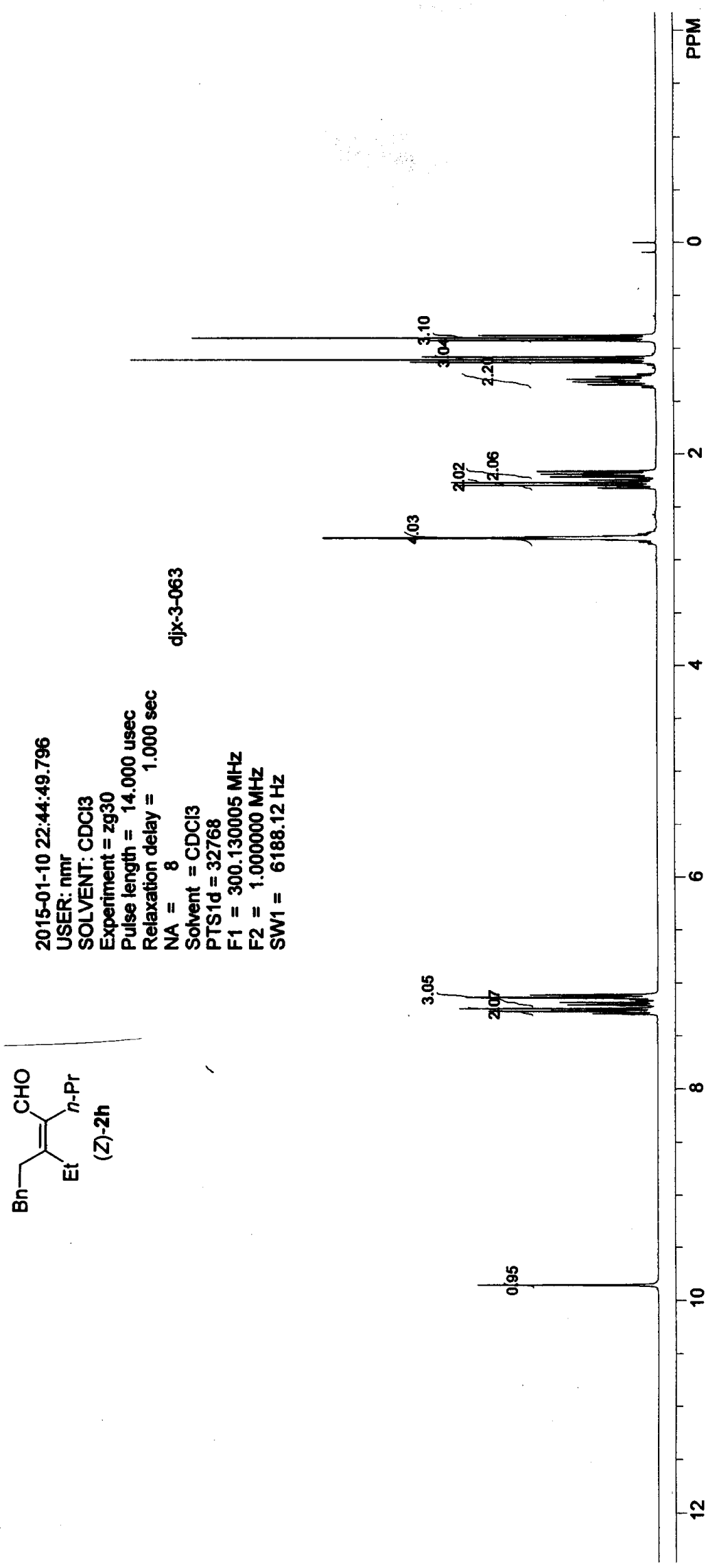

[ 
12921

s9l'tr

०६9' $z 2$

190.22

$\angle \varepsilon \varepsilon \cdot \angle Z$

†08'L

ols' $9 \varepsilon$

LLS' $9 L$

Zहम.

696 เ9เ

S6L'9ZL

s9E' 82 b

टा6 98

S6z'아

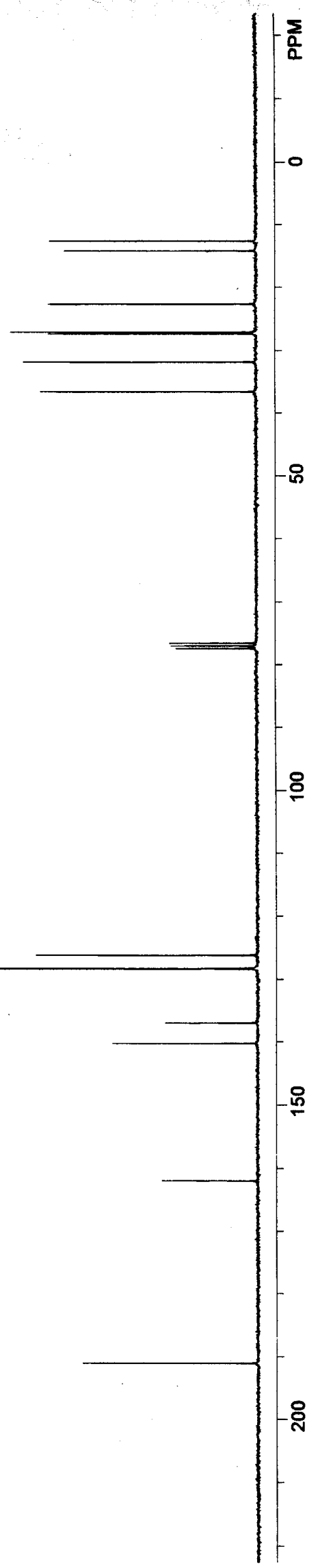



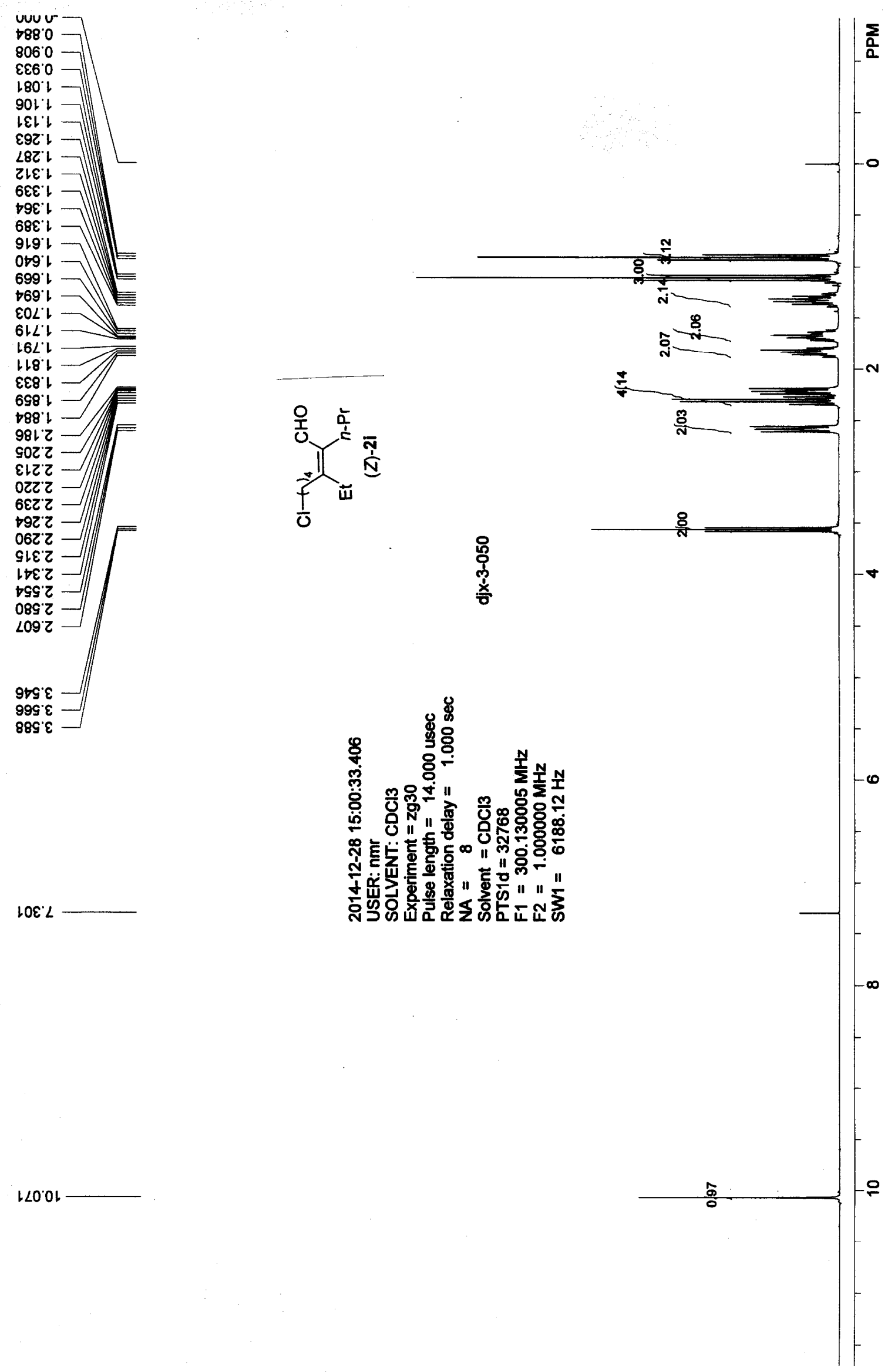
$989 \mathrm{Zl}$

99LbL

$\triangle O L Z$

GZVLL

6!t LZ

OSL $\angle Z$

6Z 62

เ8เ $2 \varepsilon$

$\angle 6 \varepsilon$

$\angle \angle S 9 L$

$0002 L$

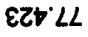

$9 g L 9 \varepsilon L$

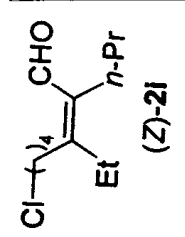

앵
ơ
뜸

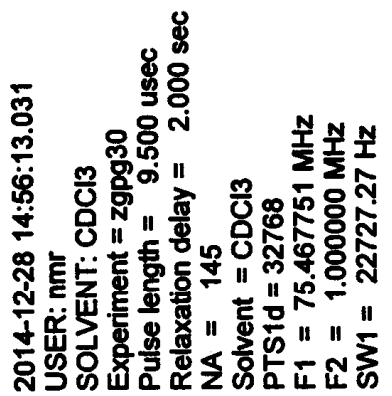

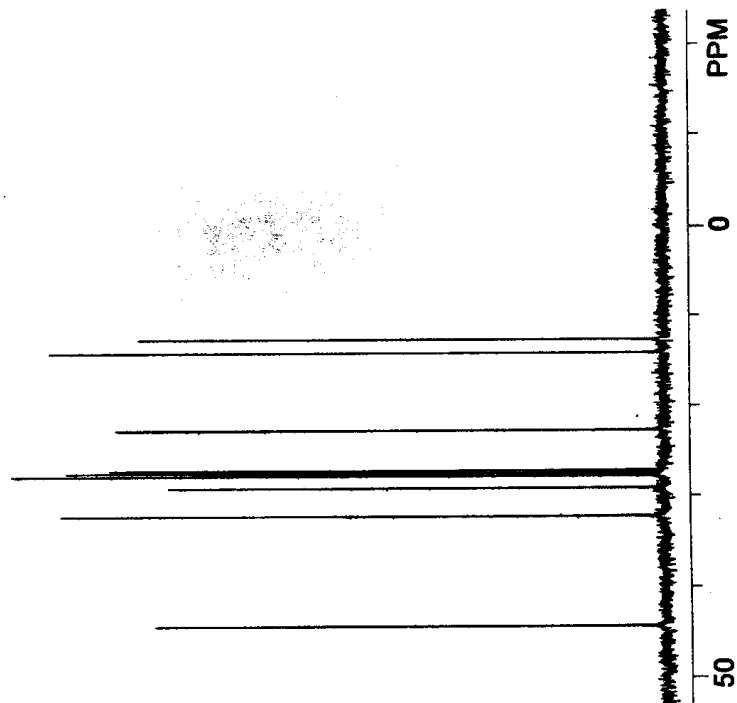

8

$26 l^{\circ 9} \varepsilon$

Lะะ เ6เ 

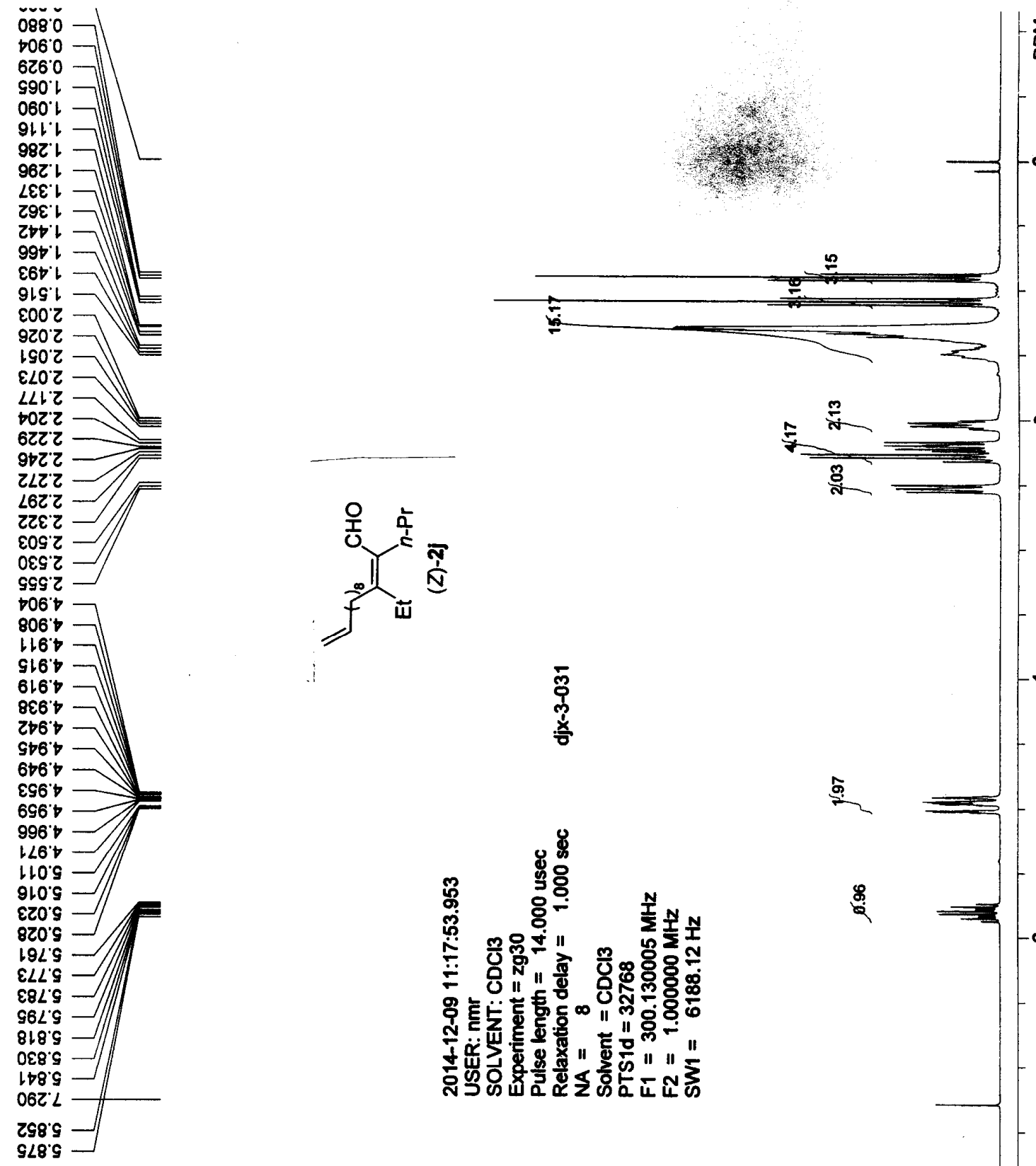

$\angle 90^{\circ} \mathrm{OL}$

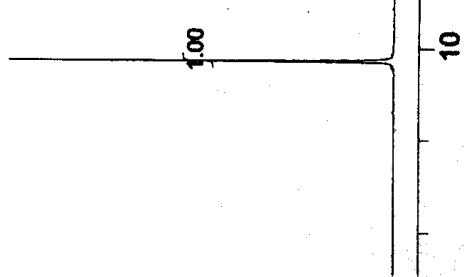



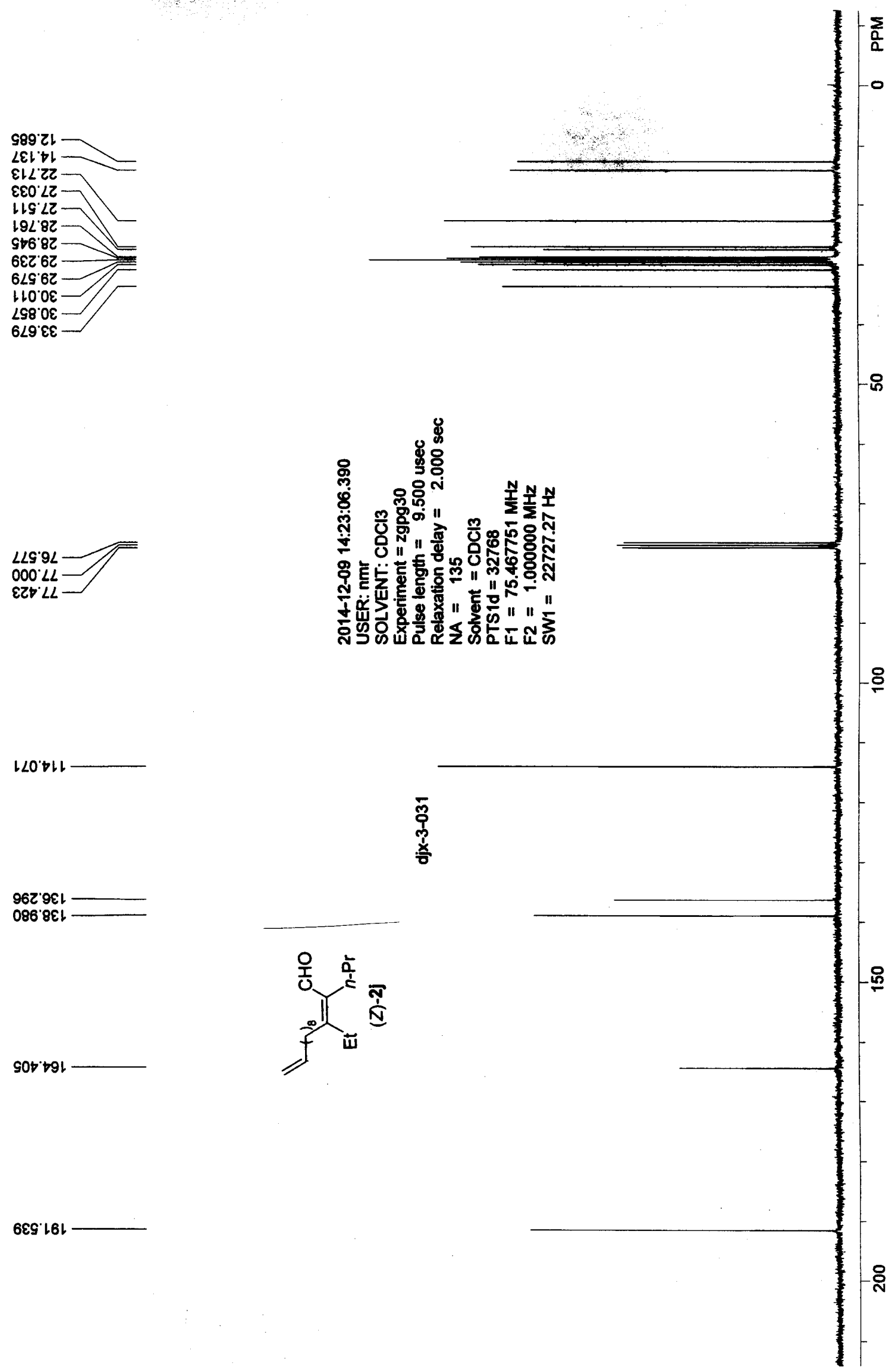

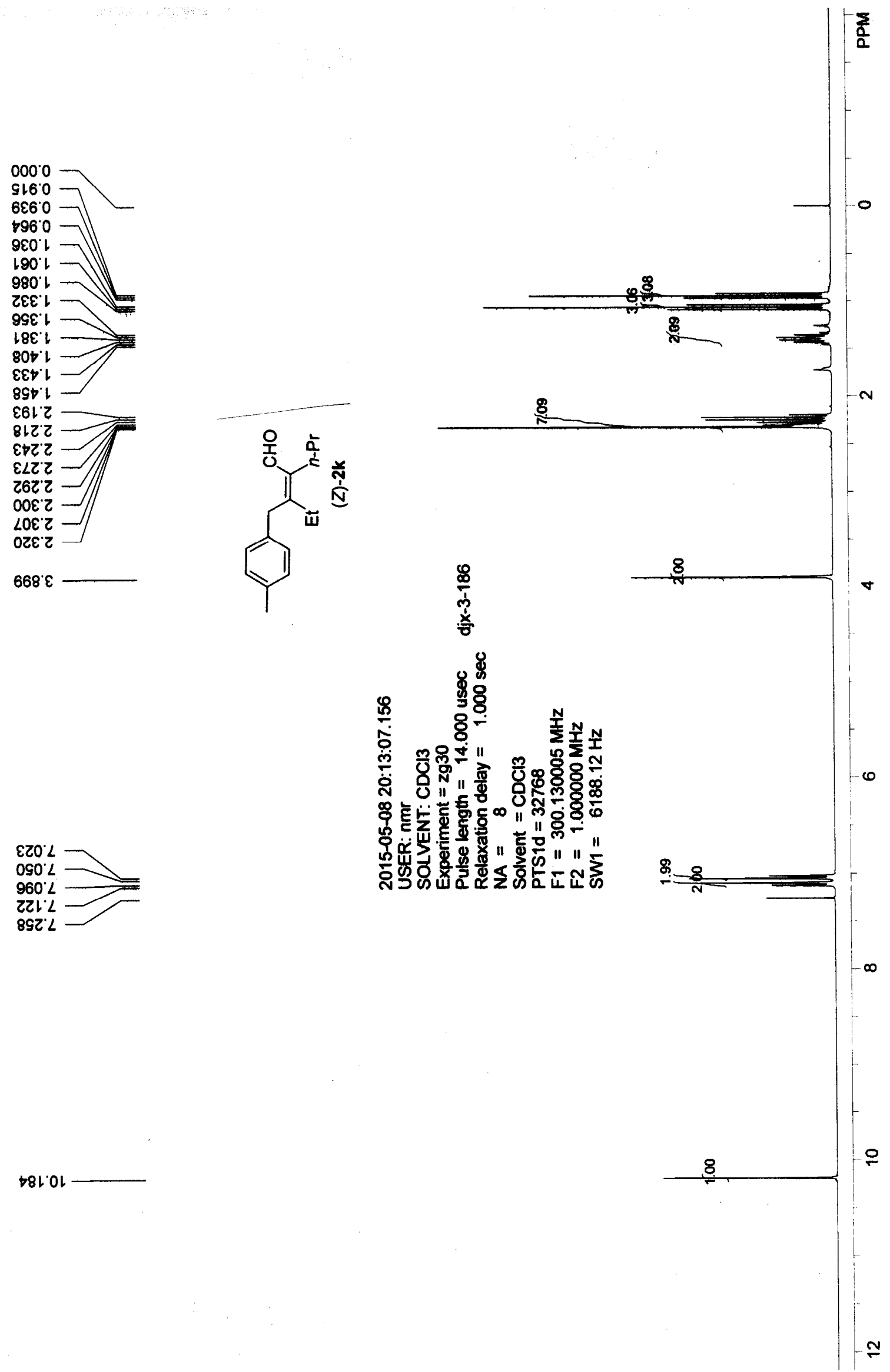

$\$ 8 \mathrm{~L}^{\circ} \mathrm{OL}$ 

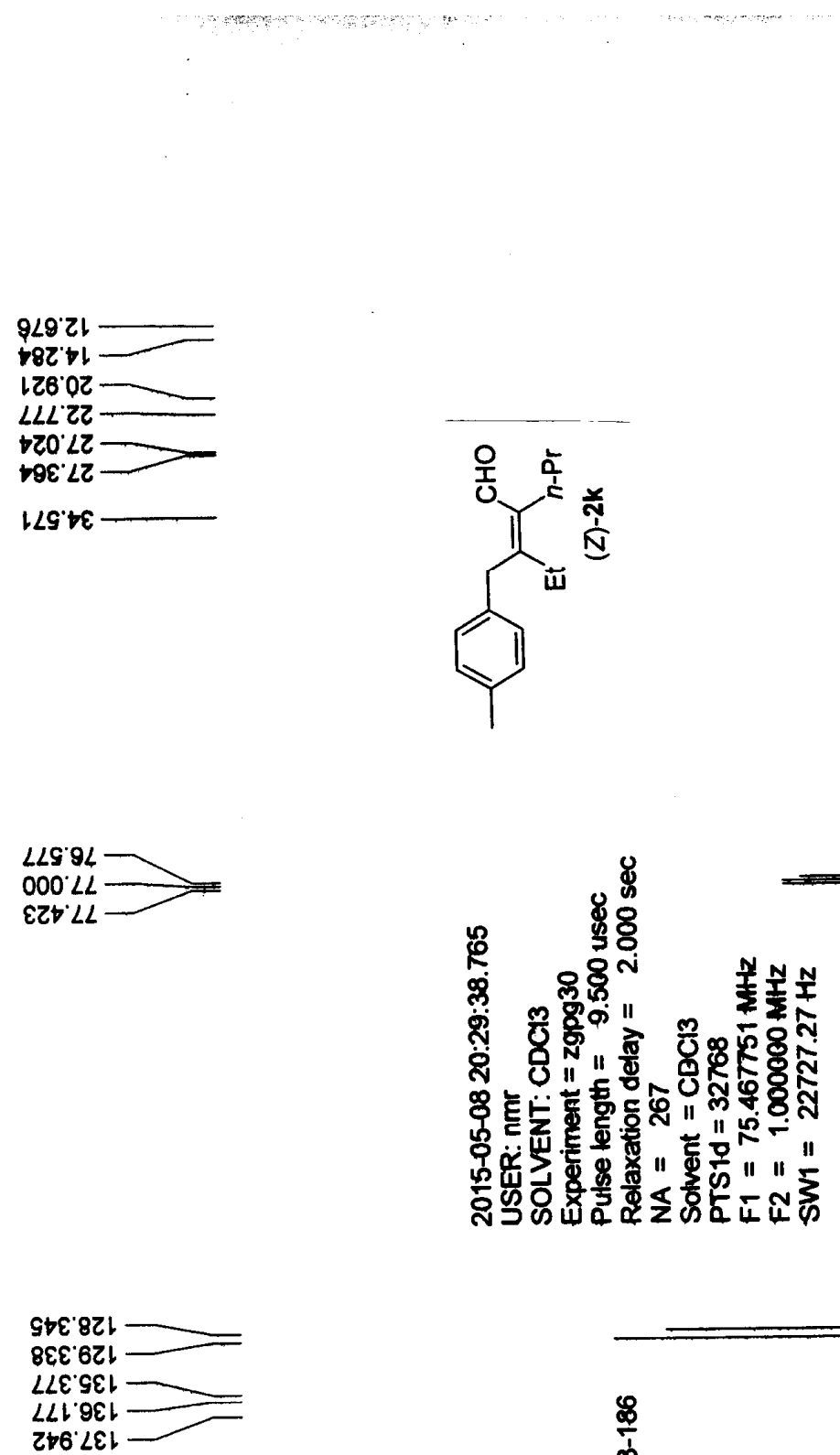

$\frac{\mathscr{8}}{\frac{1}{\dot{j}}}$

$\angle 80^{\circ} \cdot 9 l$

$800^{\circ} 261$ 

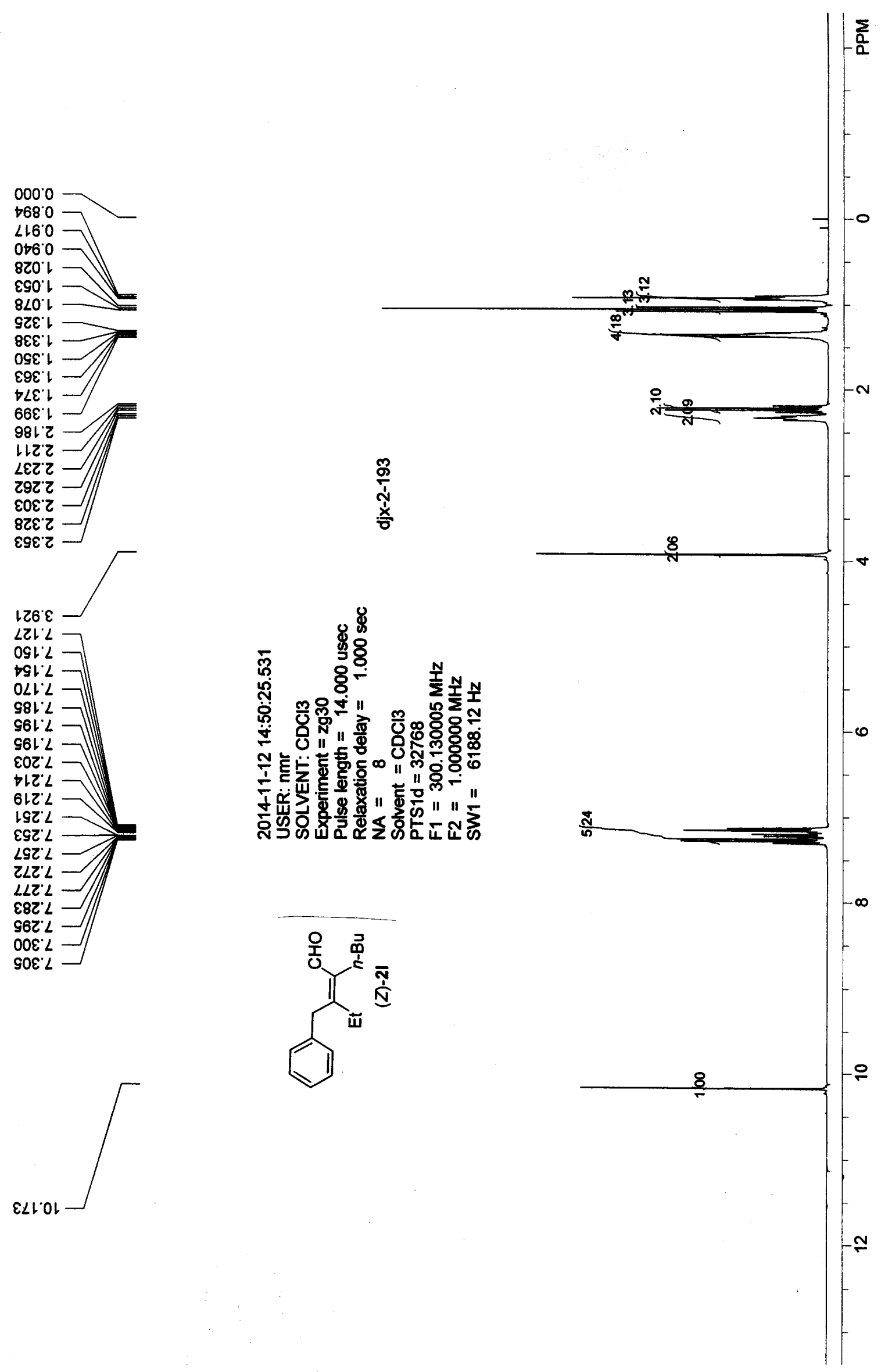


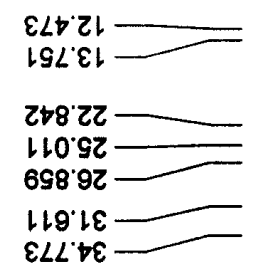

$\angle \angle G^{\prime} 9 L$

$000 \angle L$

$z \varepsilon+\angle L$
$\frac{\mathscr{O}}{\sigma}$
ஸे
$\frac{x}{\sigma}$

$\mid 15$

-
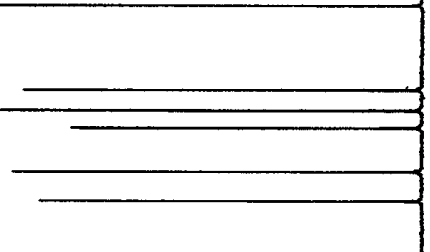

요

8

㱪

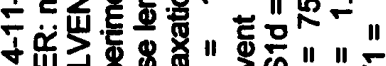

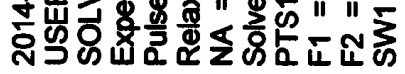

$69 \varepsilon^{\prime} 9 Z$

ع9ट 82

$0808 \varepsilon$

$6 เ \varepsilon^{\prime} 8 \varepsilon$ เ

$891^{\circ} 091$

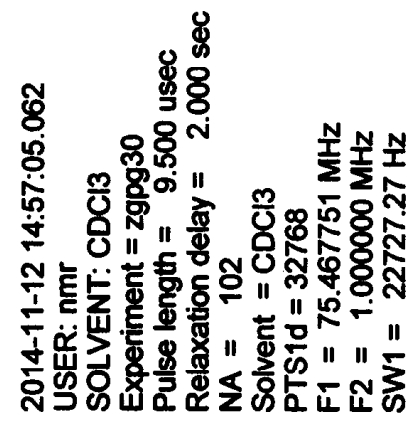

요<smiles>C=CC(=C)C(C)=C(C=C)Cc1ccccc1</smiles>

$689 '\llcorner 6 \downarrow$

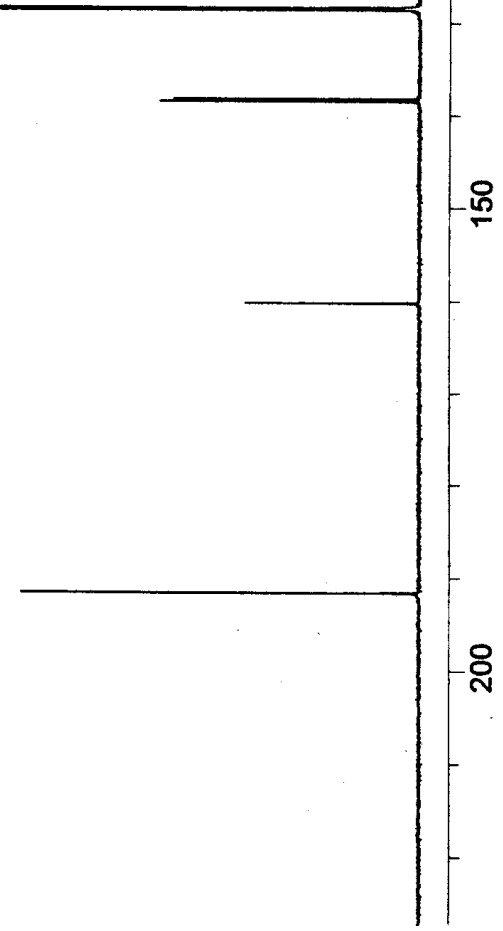



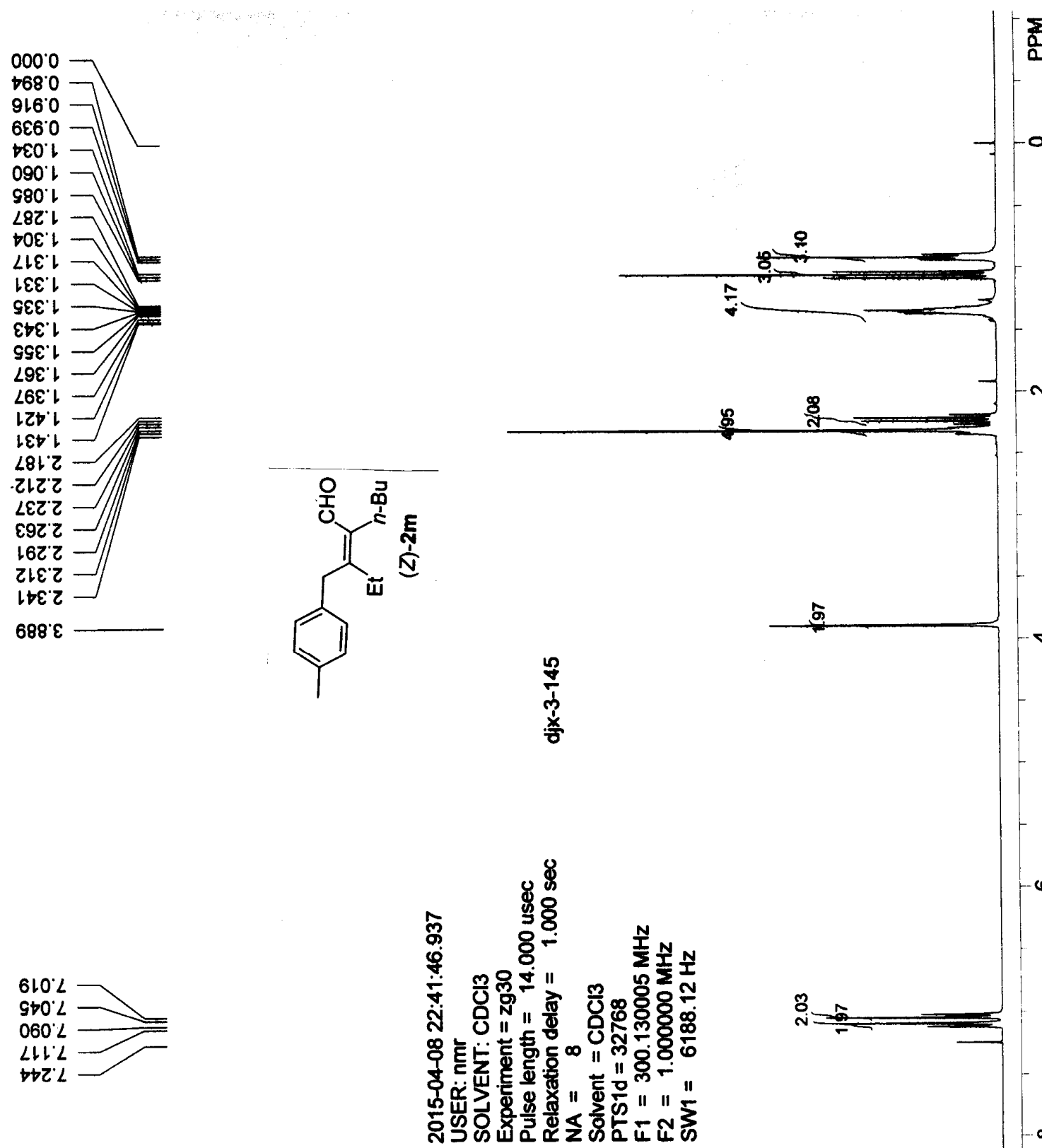

$6 \angle 1 \circ 2$

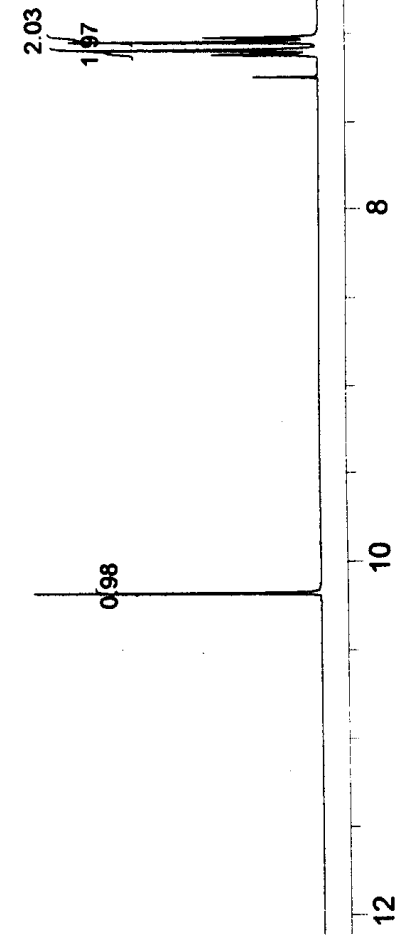



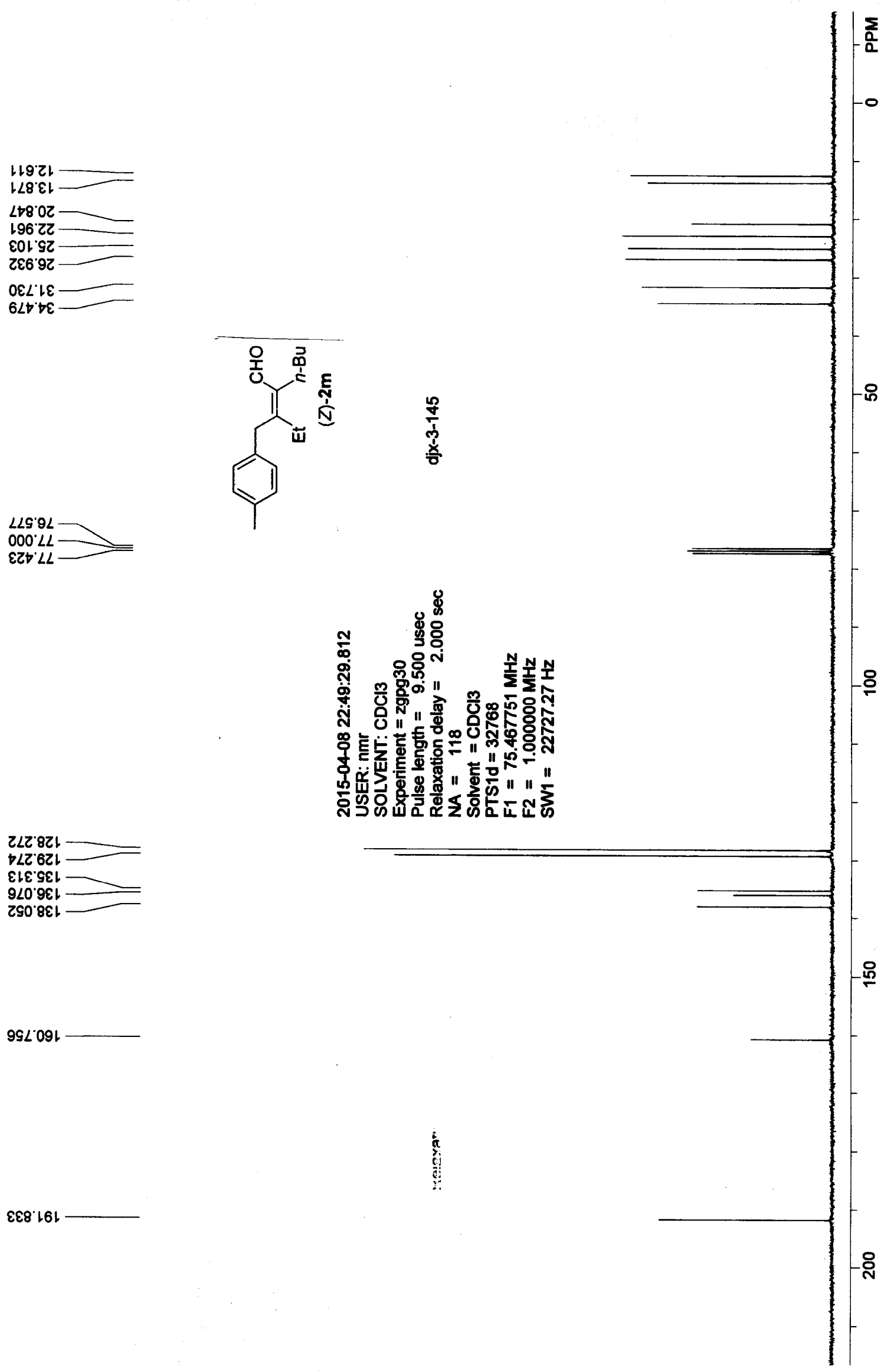

$Z \angle Z 8 Z 1$
$\checkmark \angle Z 6 Z 1$

EเE'GE

9L0 $98 \mathrm{~L}$

Z90'8ह

$99 L 09$ 

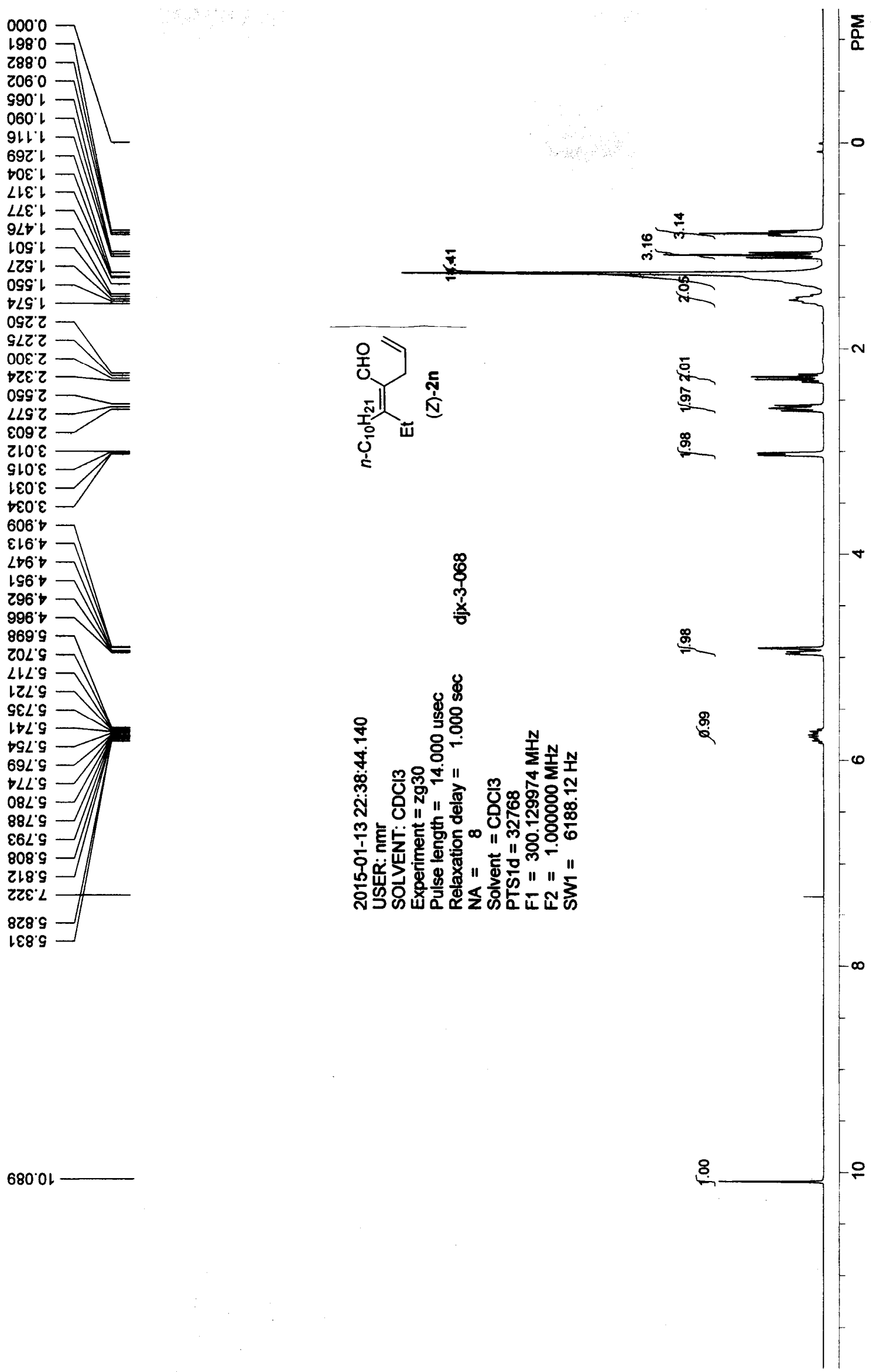
9๕๕乙レ

Lᄂ6'

2092

$\angle 992$

$\angle 08^{\circ} 82$

$8 \varepsilon L^{\prime} 6 \mathrm{Z}$

เZZ 62

$69 \varepsilon 62$

$96 \varepsilon^{\prime} 6 Z$

6LS'6Z

BSLOE

BEL'OE

ZLLLE

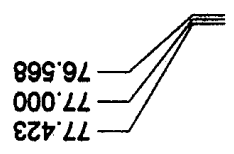

$282 \bullet \downarrow$

\&̊
ફे
츰

6เЬ $\varepsilon \varepsilon l$

OO० $S E L$

$6 \varepsilon 8$ ' 9 เ

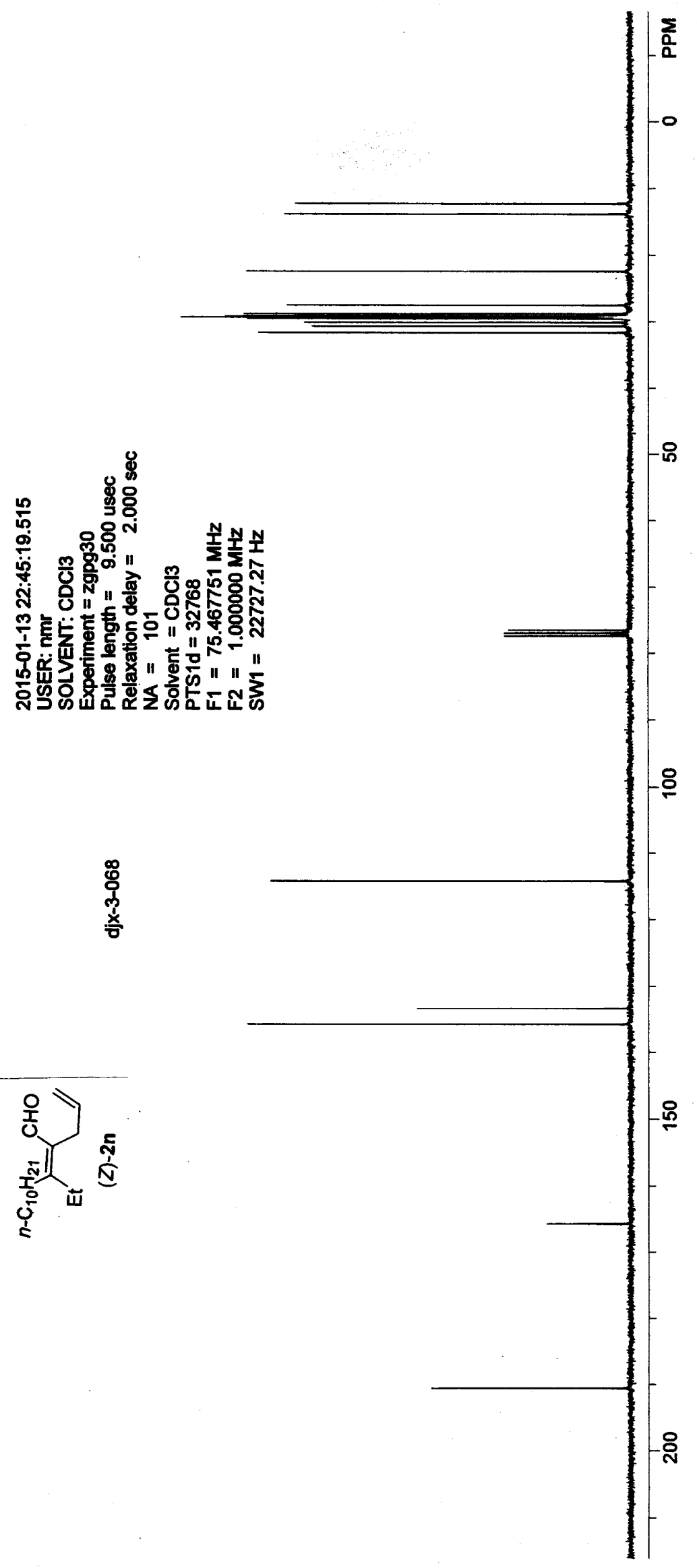



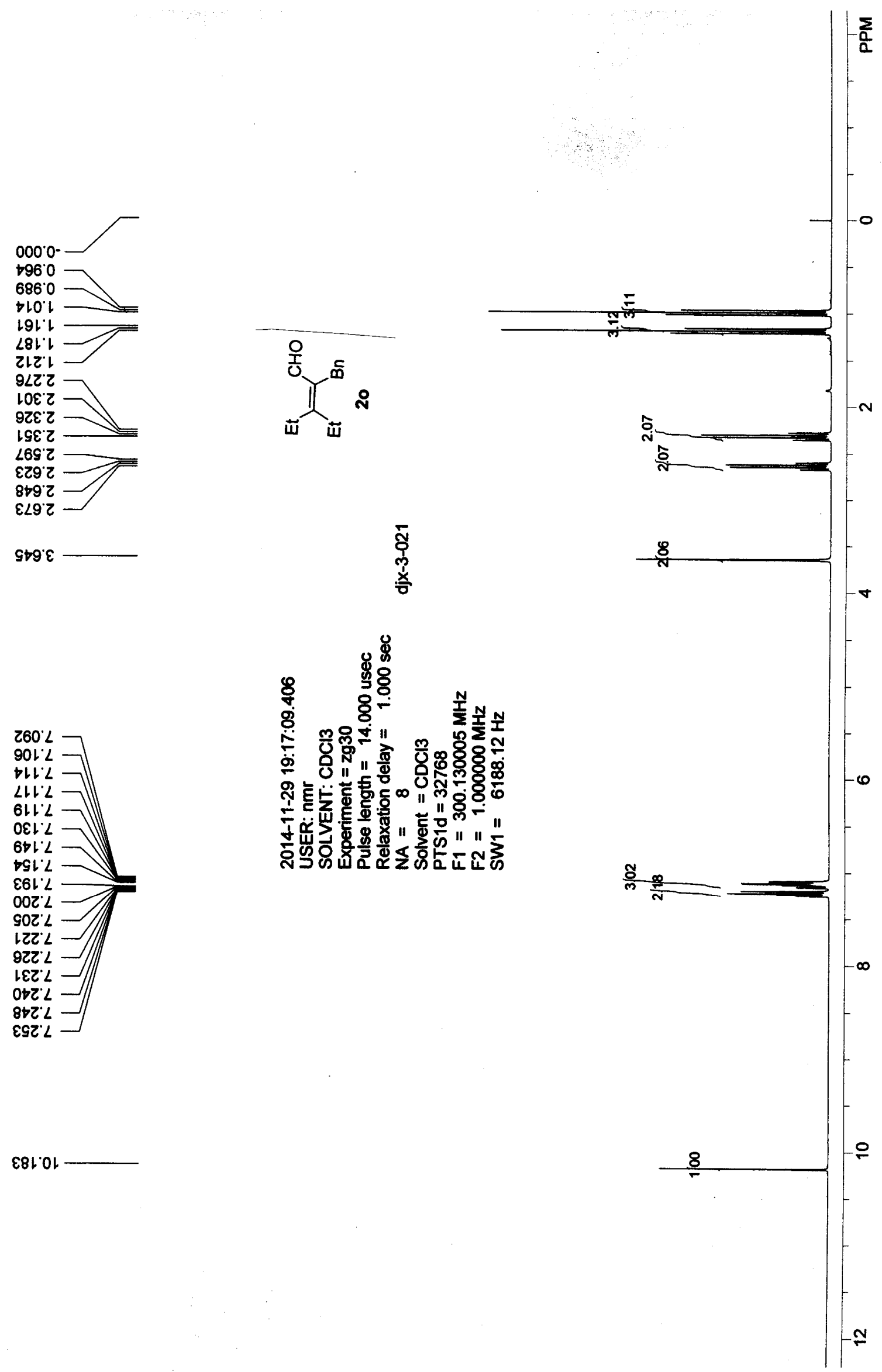

E8เ०O 

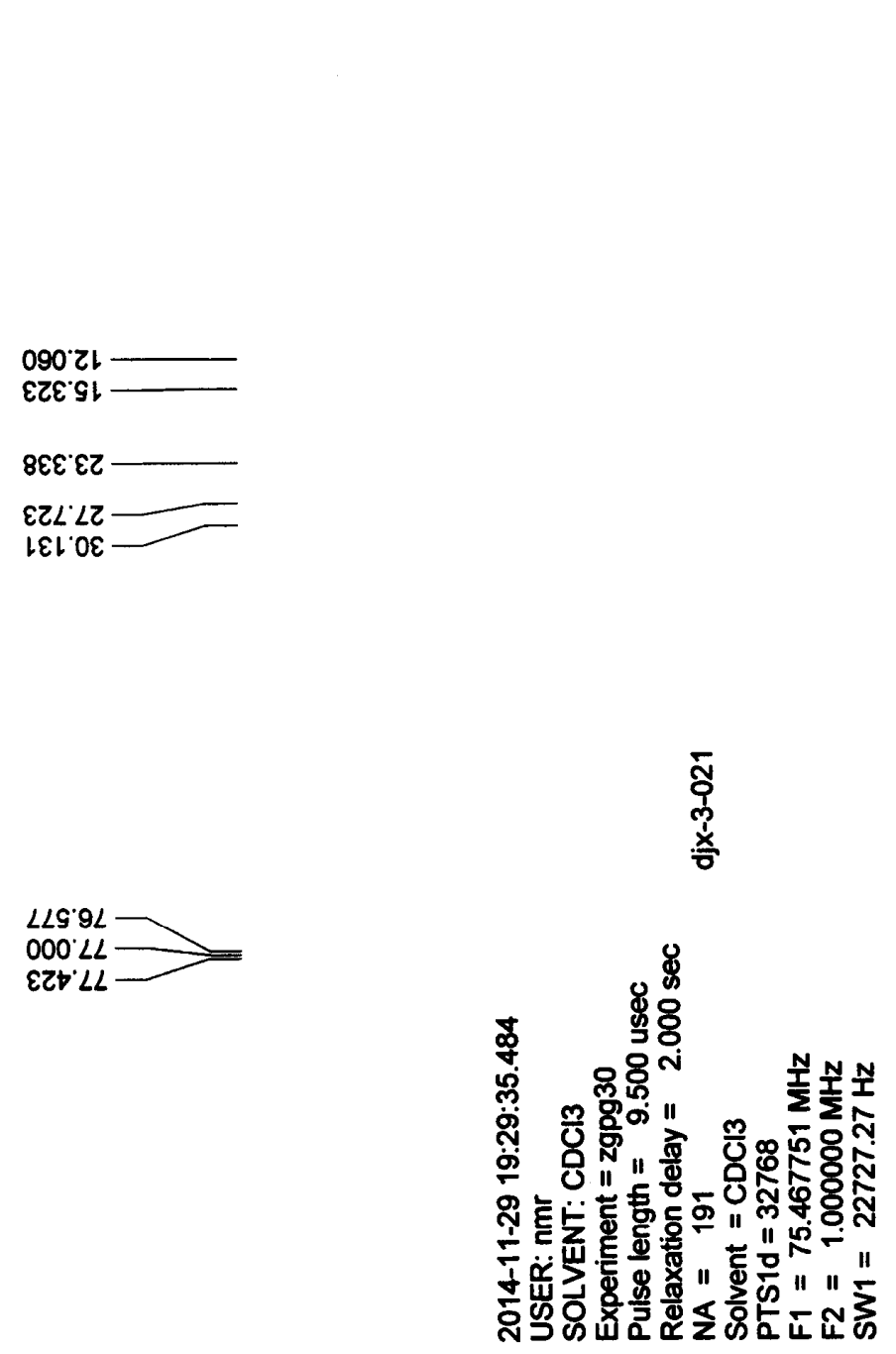

†\&9'G乙 -

$\angle L 8$ LZL 681.821

ट०६॰६เ

EOz'0t

$69 L \angle 9 l$

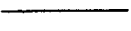

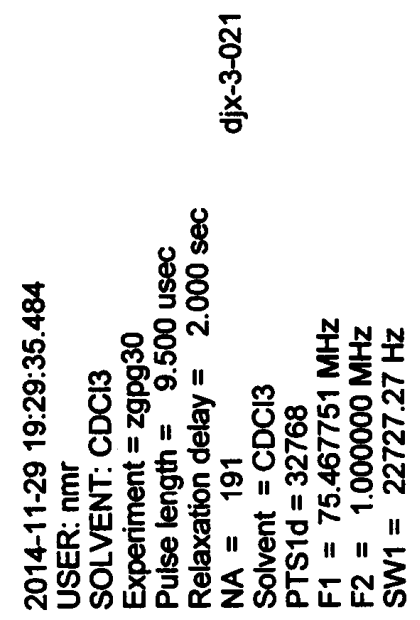

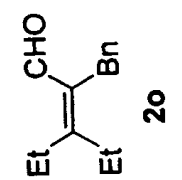

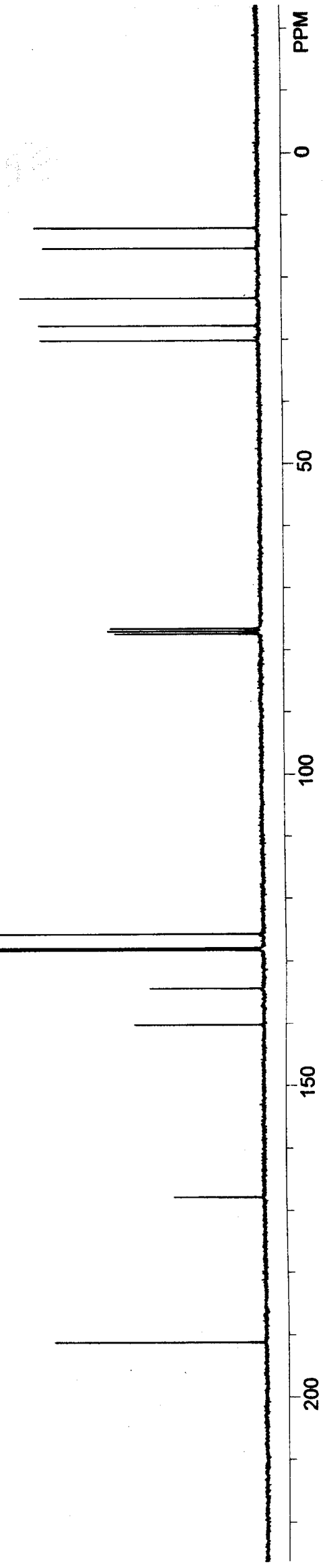

요

오 

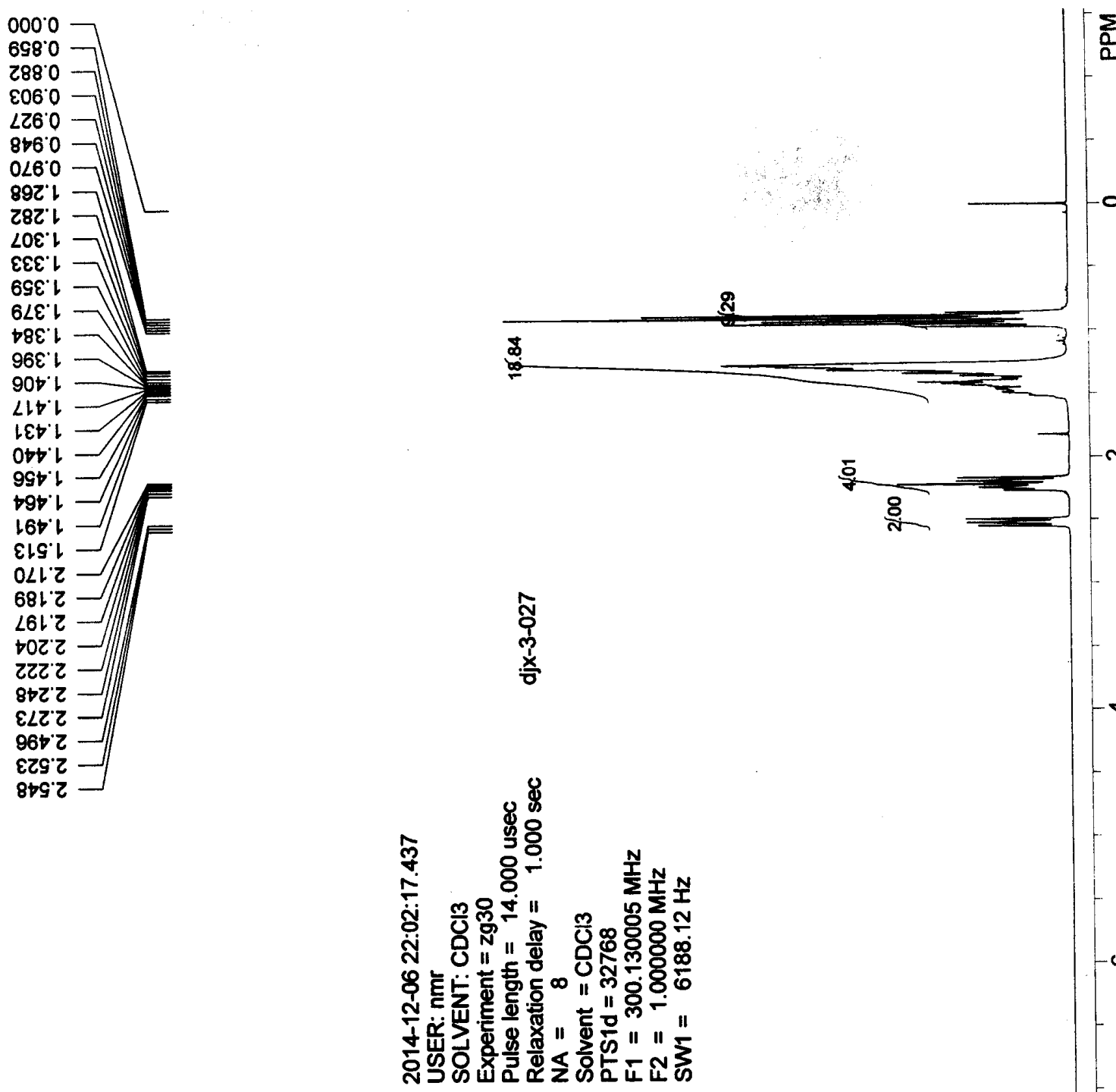

$98 Z^{\circ} L$

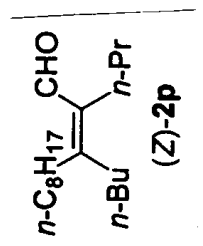

990 아 

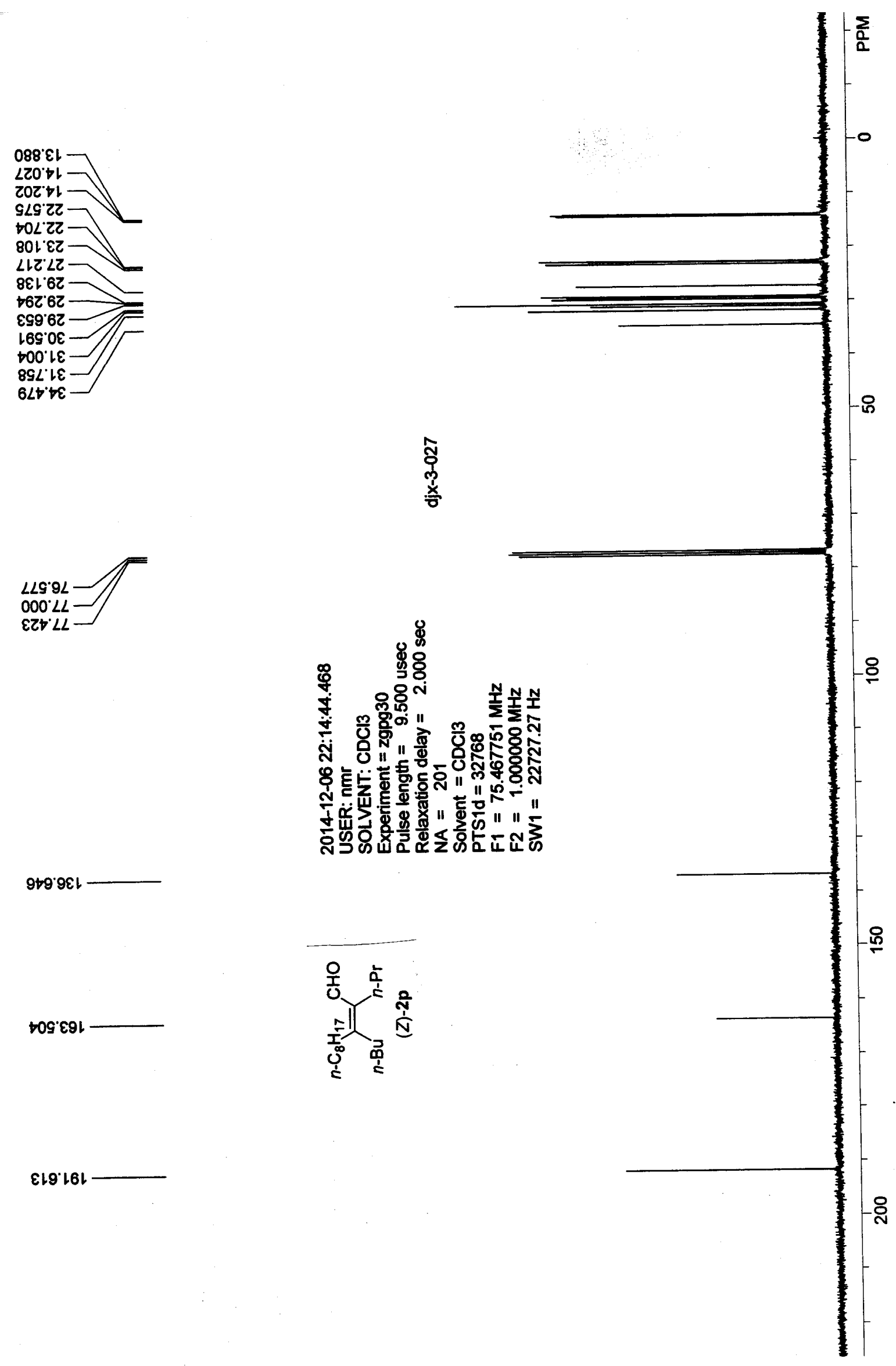

tog'ع9l -

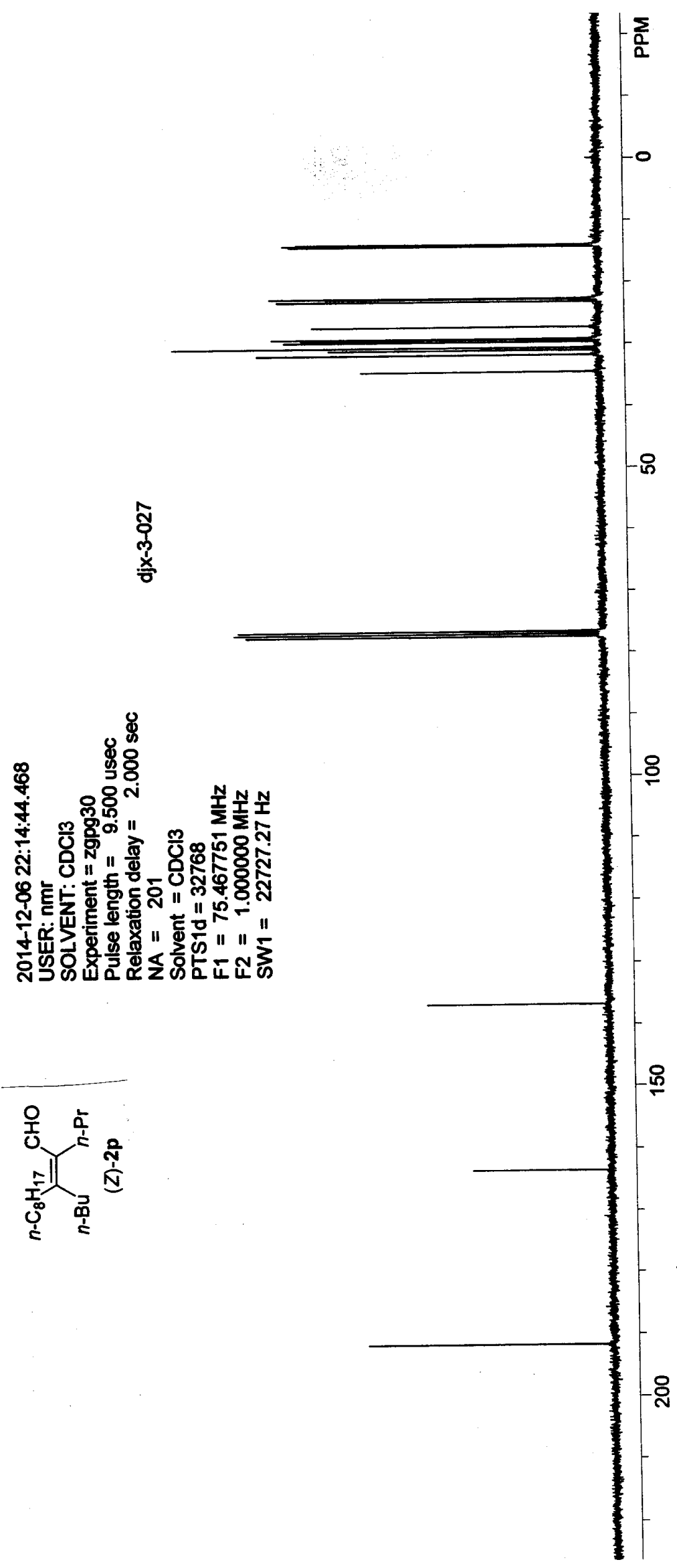

$\varepsilon เ 9^{\prime}$ เ6เ

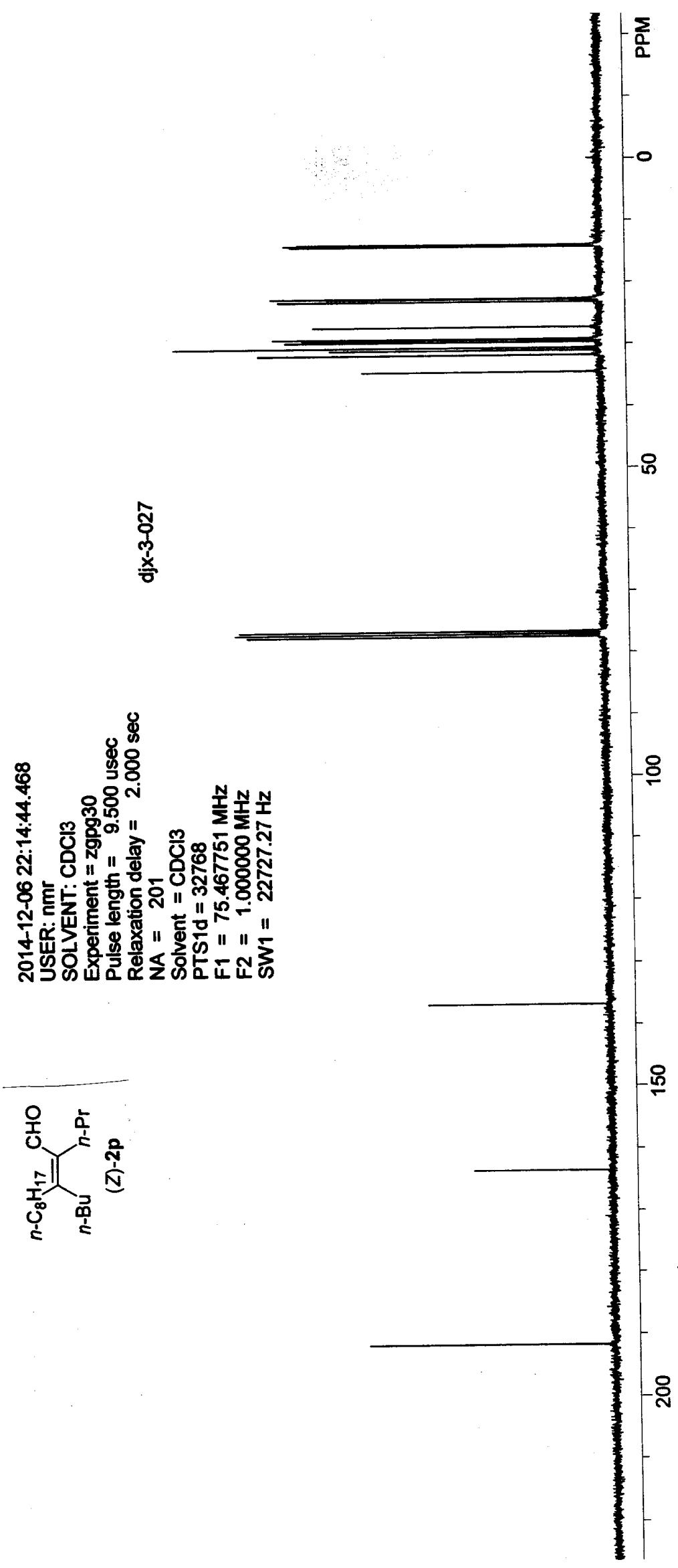

9t9.9ع।

$000.2 L$ 

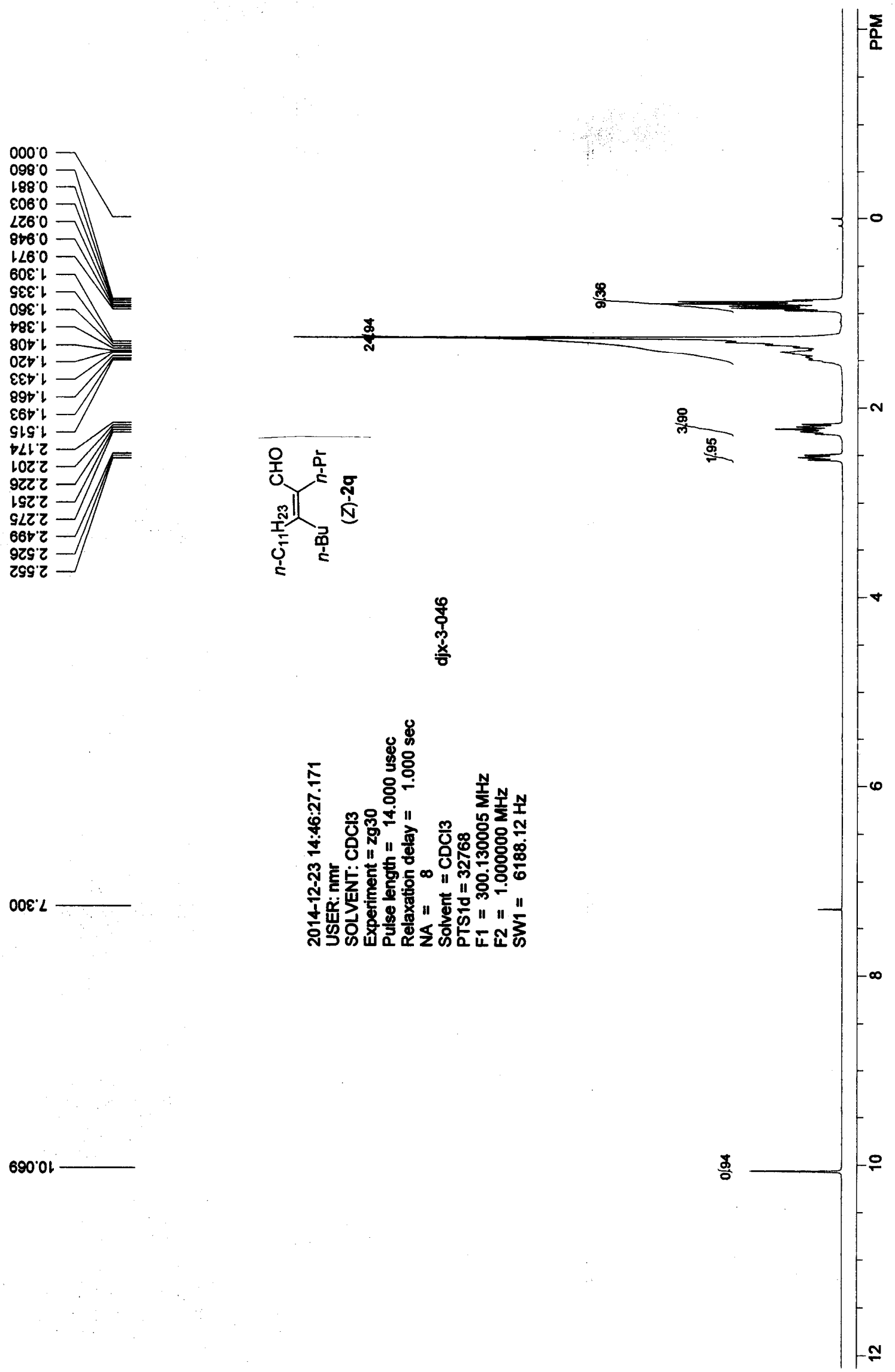

$690^{\circ} 01$ 


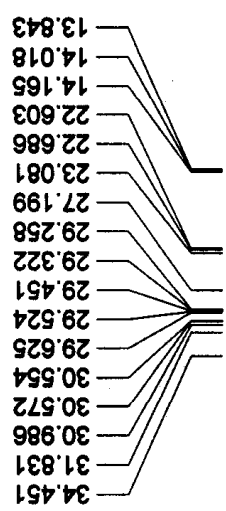

$\mid-\frac{\sum_{0}}{a}$

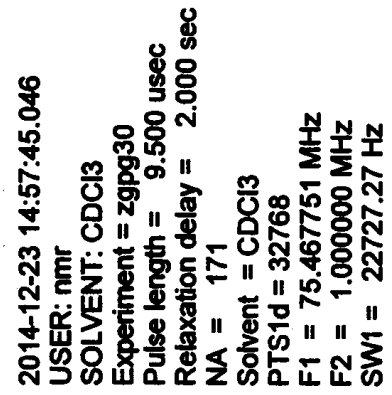

$9<\varepsilon$ ฉ9เ

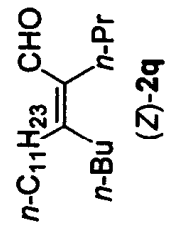

$\left|29^{\prime}\right| 6 \downarrow$

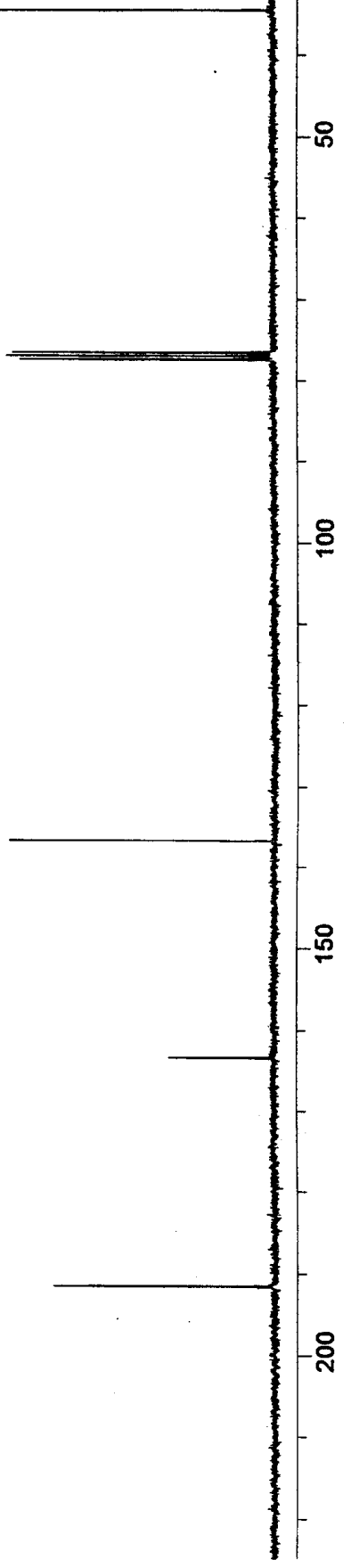



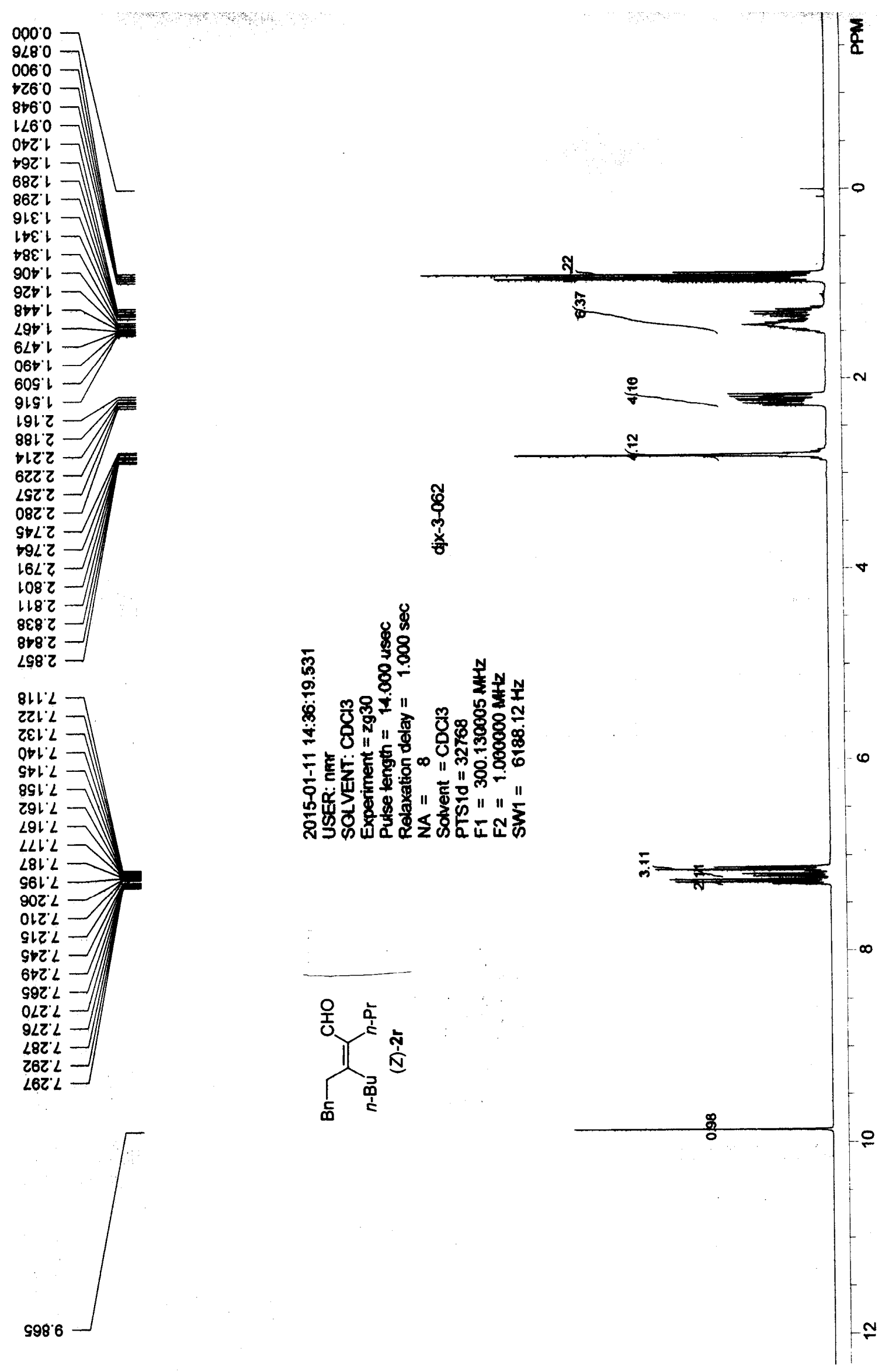

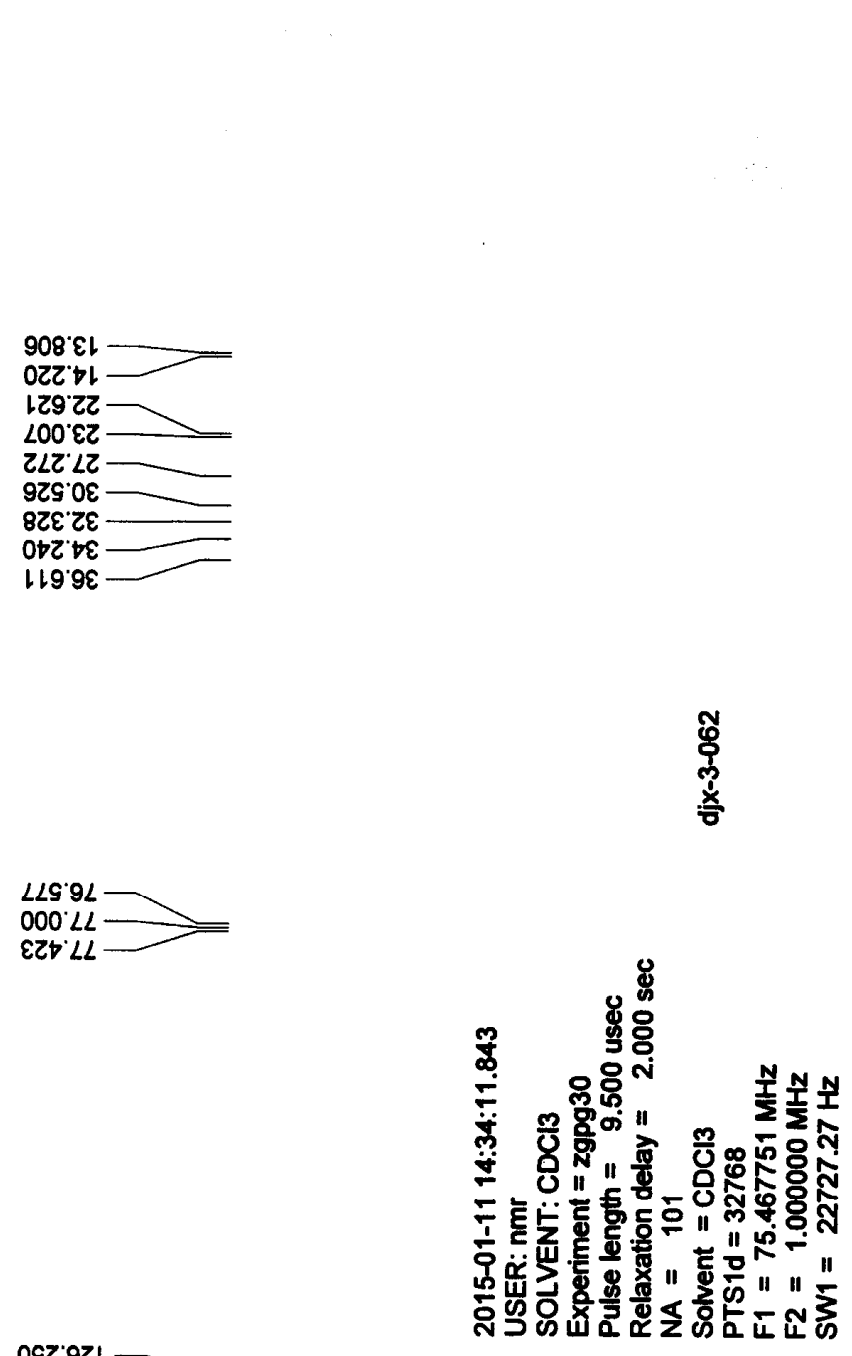

OSZ $9 Z 1$

$8 เ \varepsilon \cdot 82$

$\angle \angle G^{\prime} 9 L$

$000 \% L$

$\varepsilon Z 7 L L$

$16 \varepsilon \cdot 821 \longrightarrow$

$86 Z$ ' $\angle E L$

698'Ot

216.091
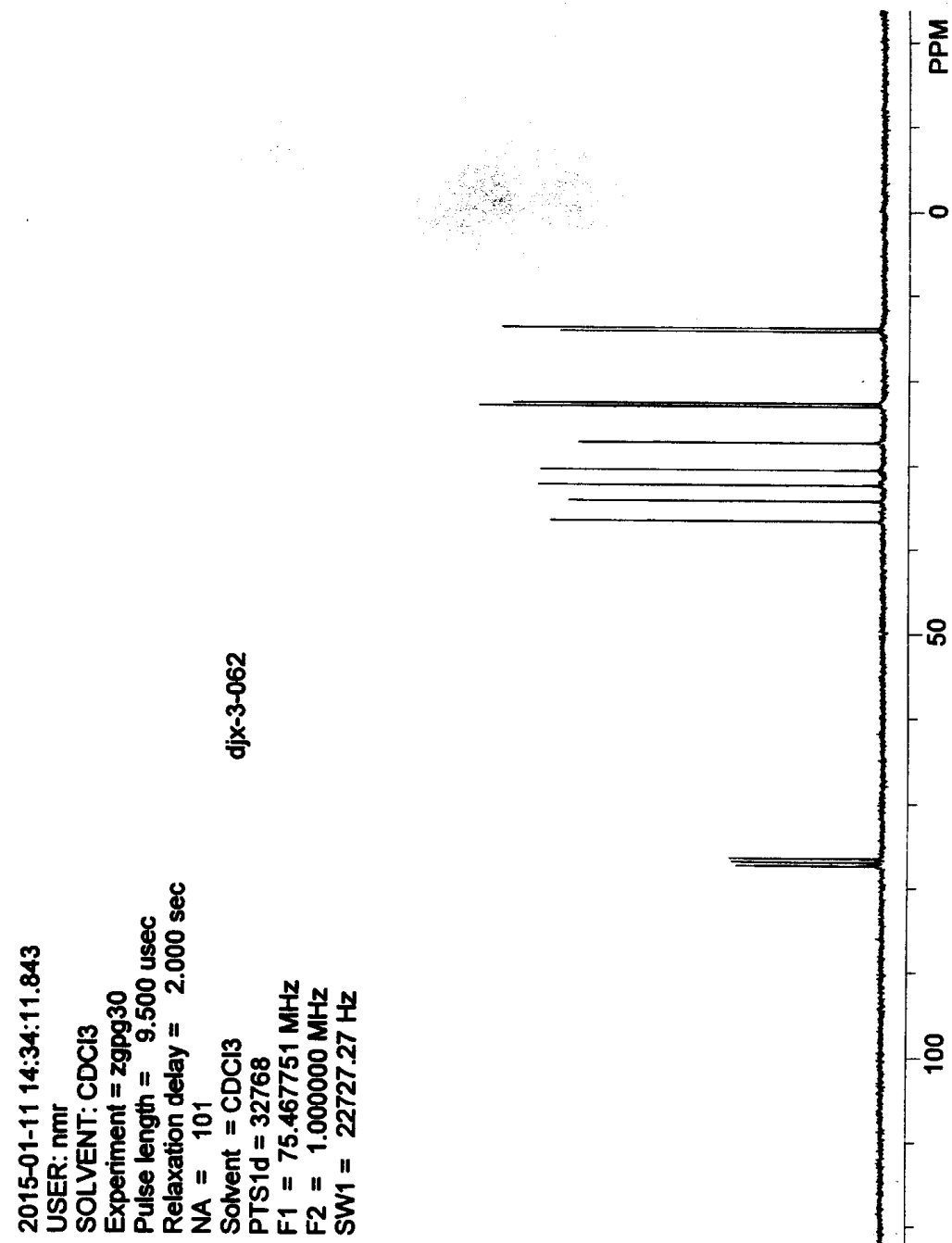

우

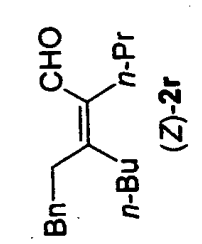

$190^{\prime} L 61$

오 

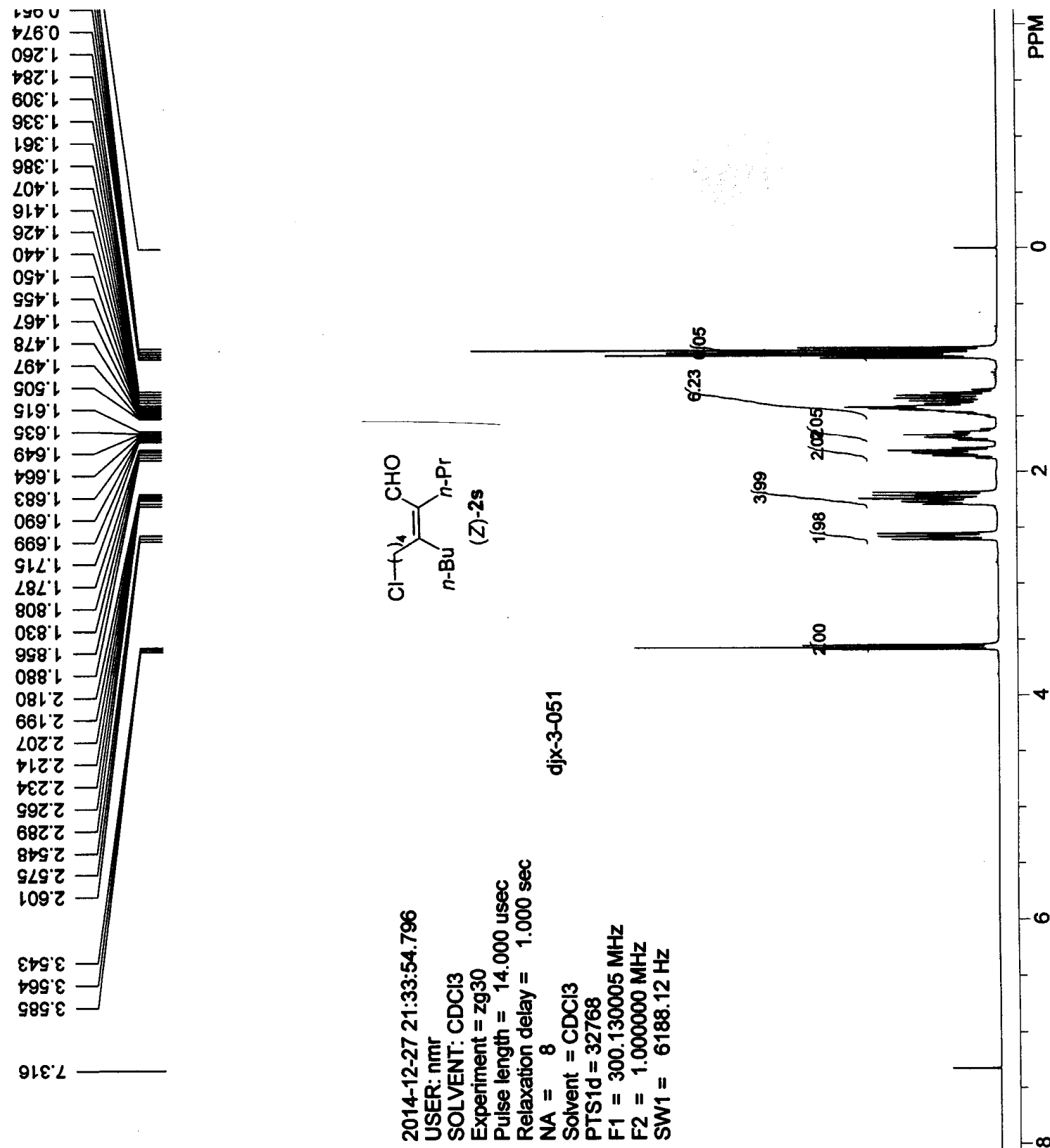

$890^{\circ}$ ㅇ

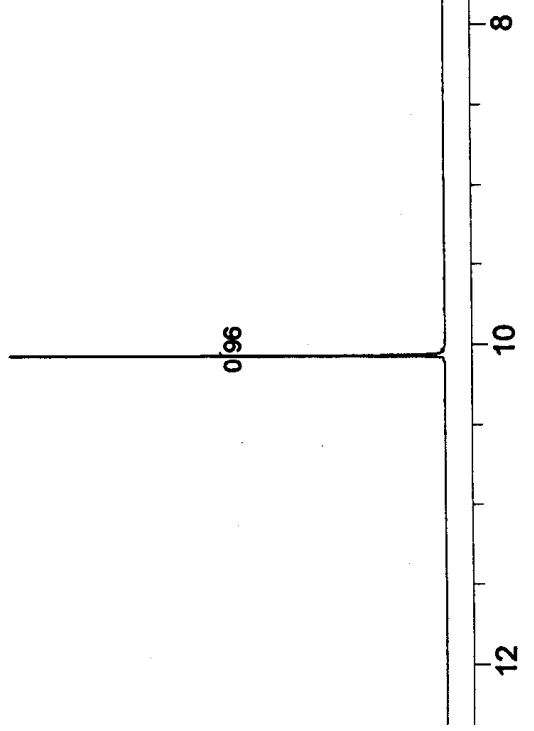




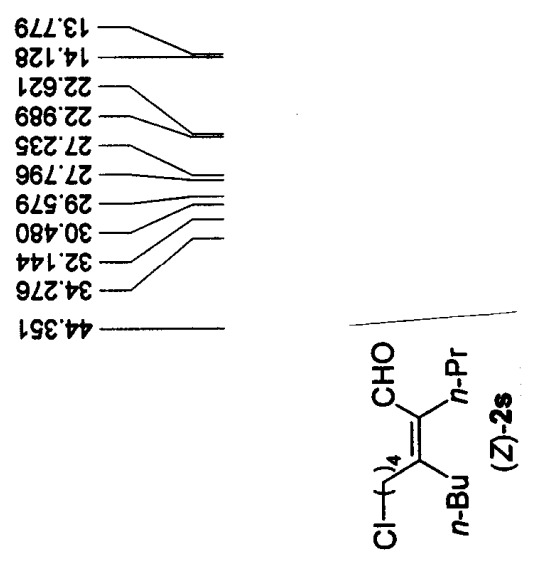

LLG' $9 L$

$000 \mathrm{LL}$

$\varepsilon Z t ' L L$

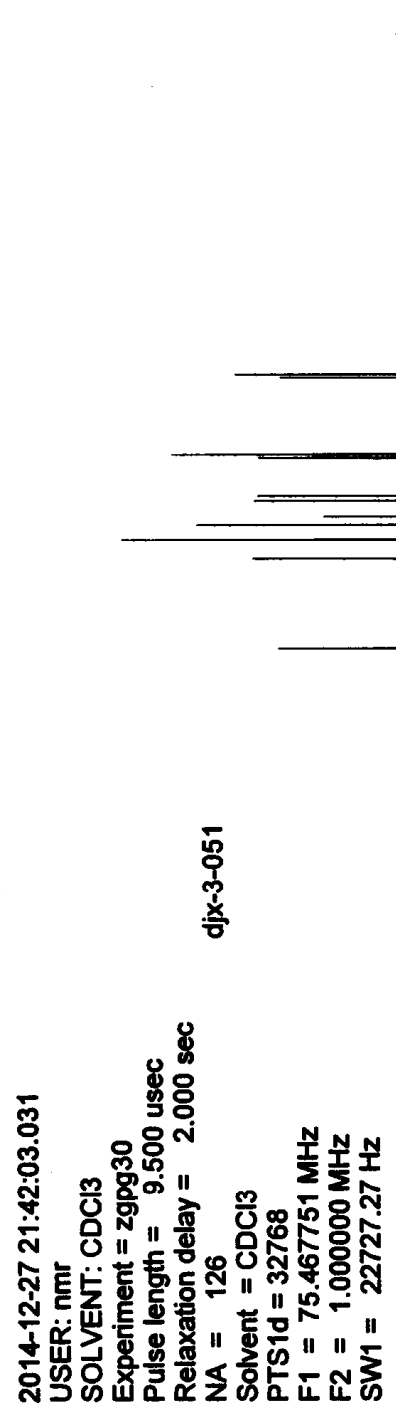

$\varepsilon Z 0^{\circ} \angle \varepsilon\llcorner$
$\varepsilon \neq 0 \cdot 29 \downarrow$

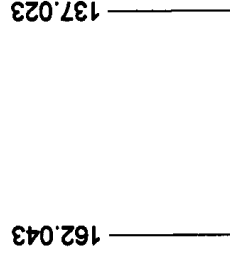

$802 \cdot 61$

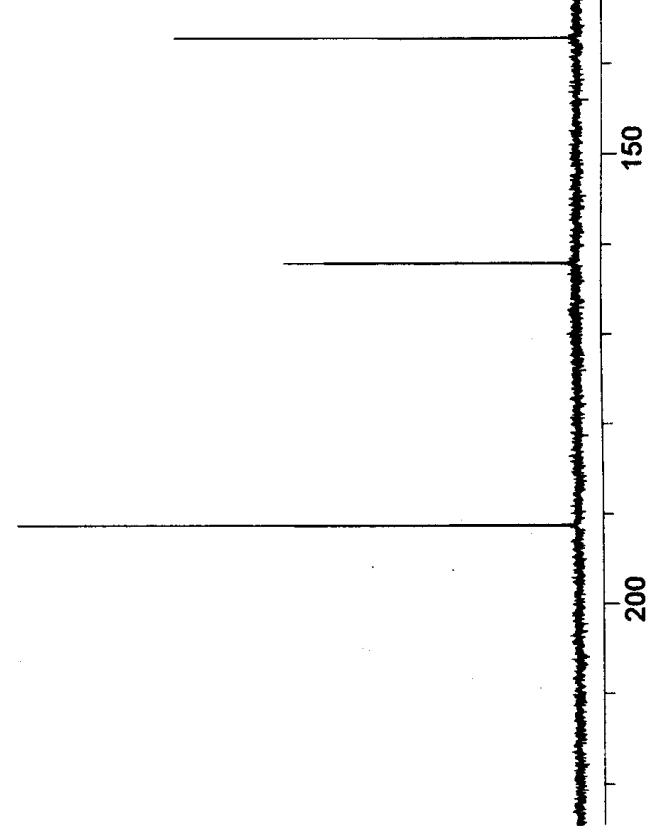



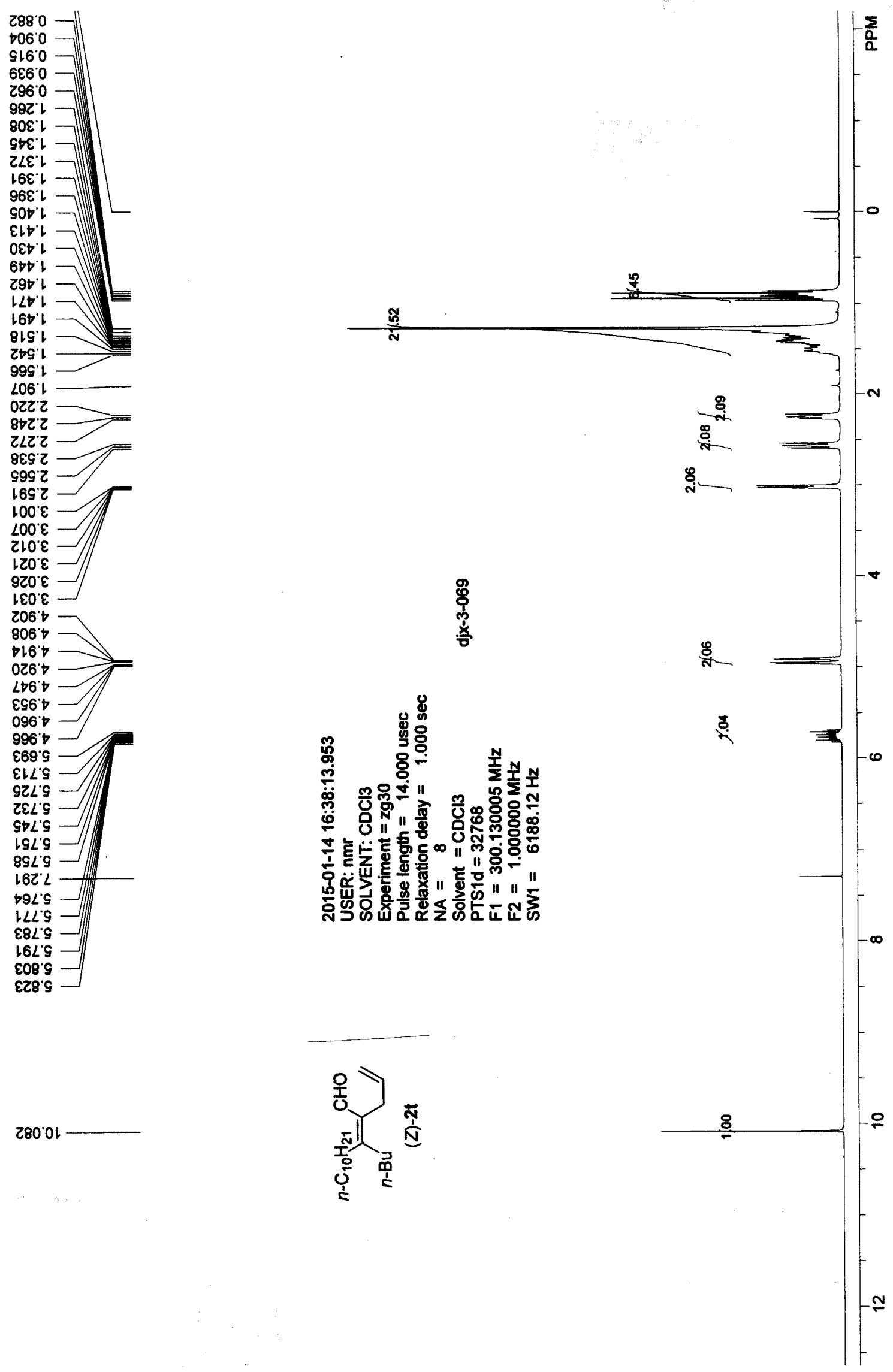

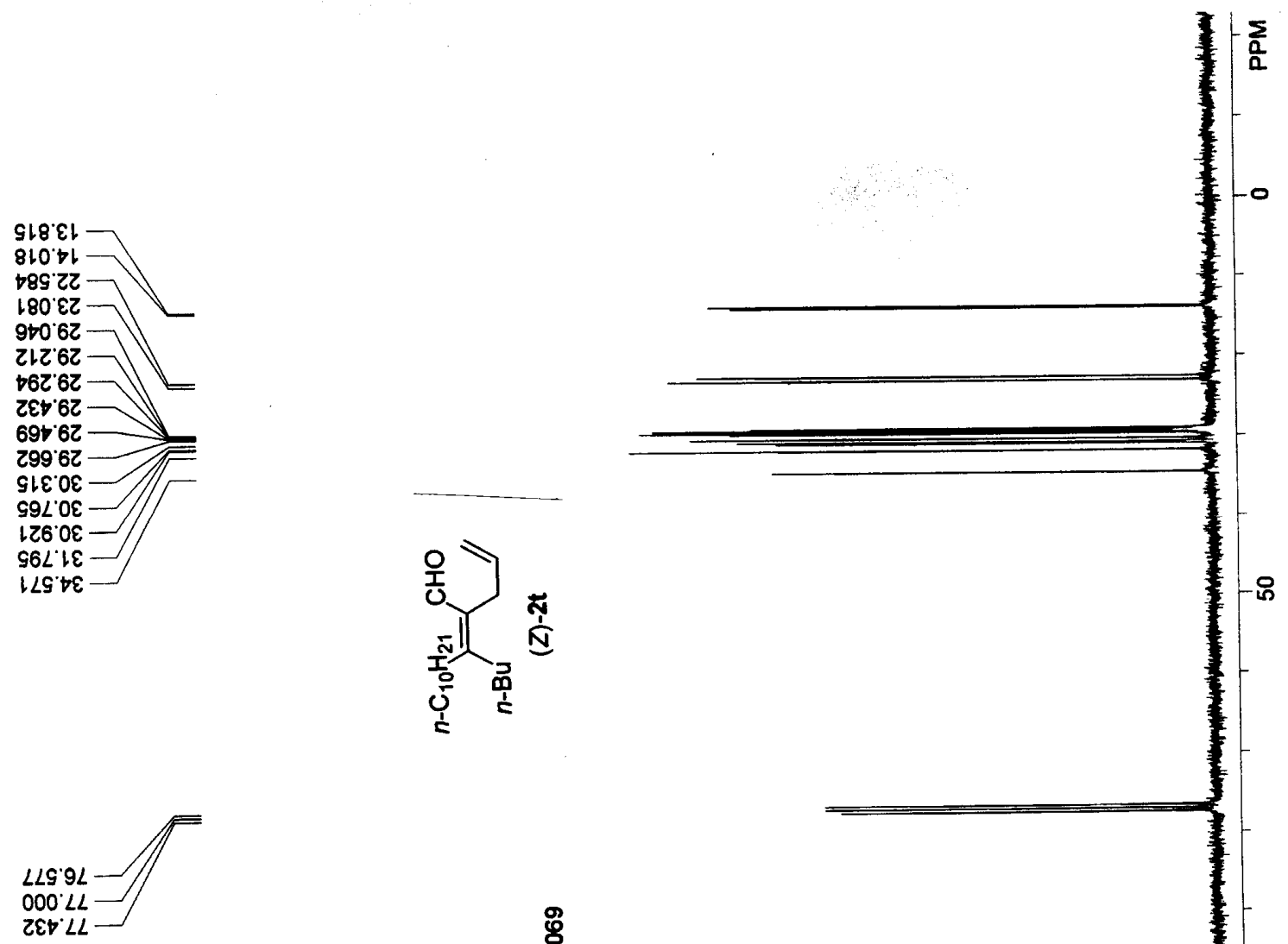

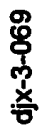

26\&เレ

$8 \angle L \varepsilon \varepsilon L$

Z68'GEL

$8+0.991$

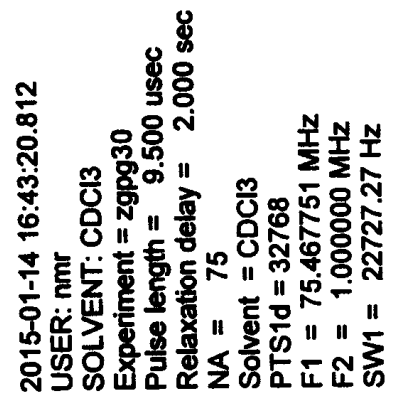

L9L 061

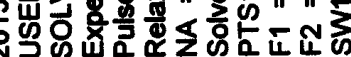



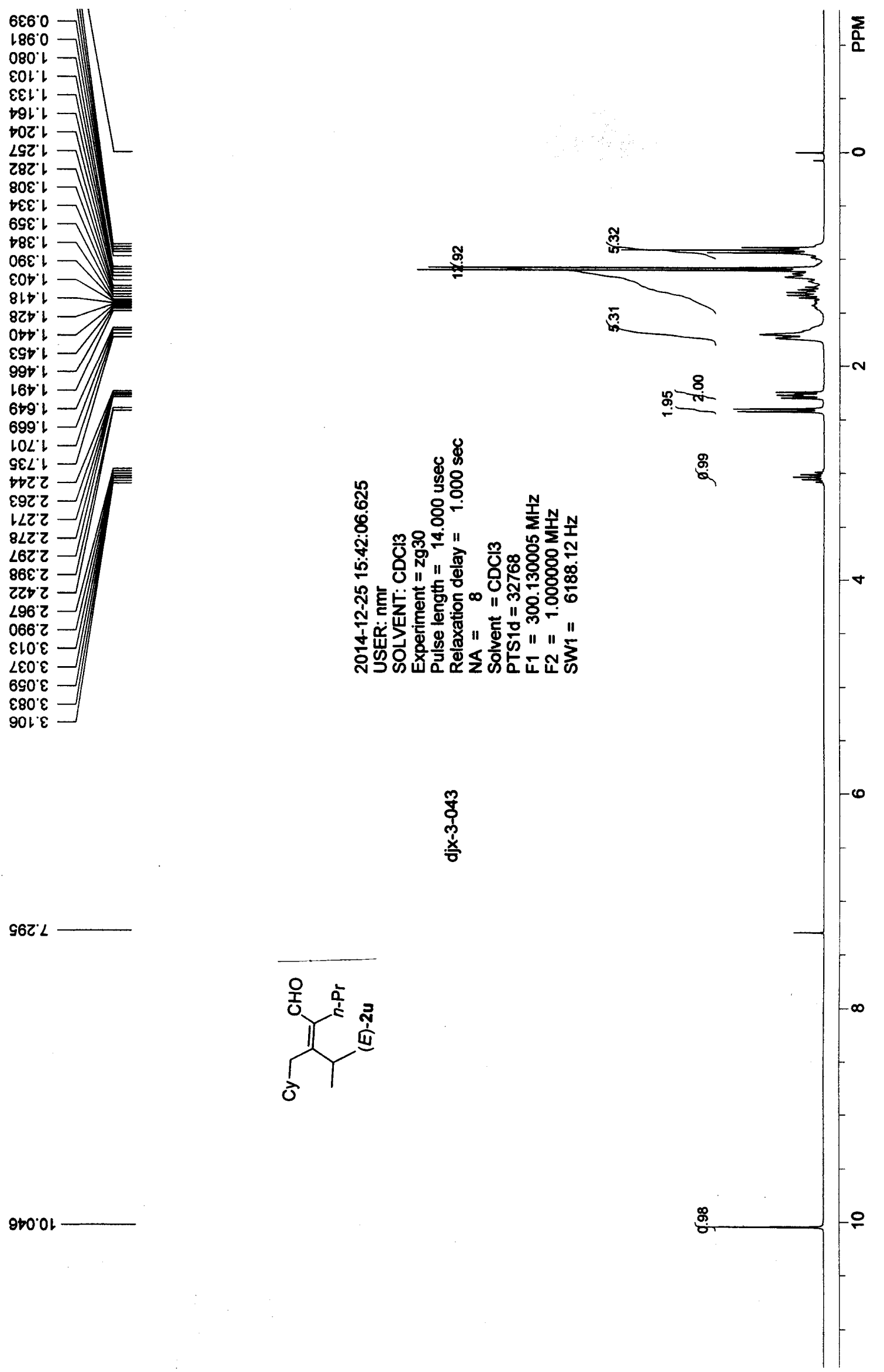

S6Z L<smiles>CC(C)C([O-])=C(C[O])C(C)C</smiles>

$9+0.01$ 

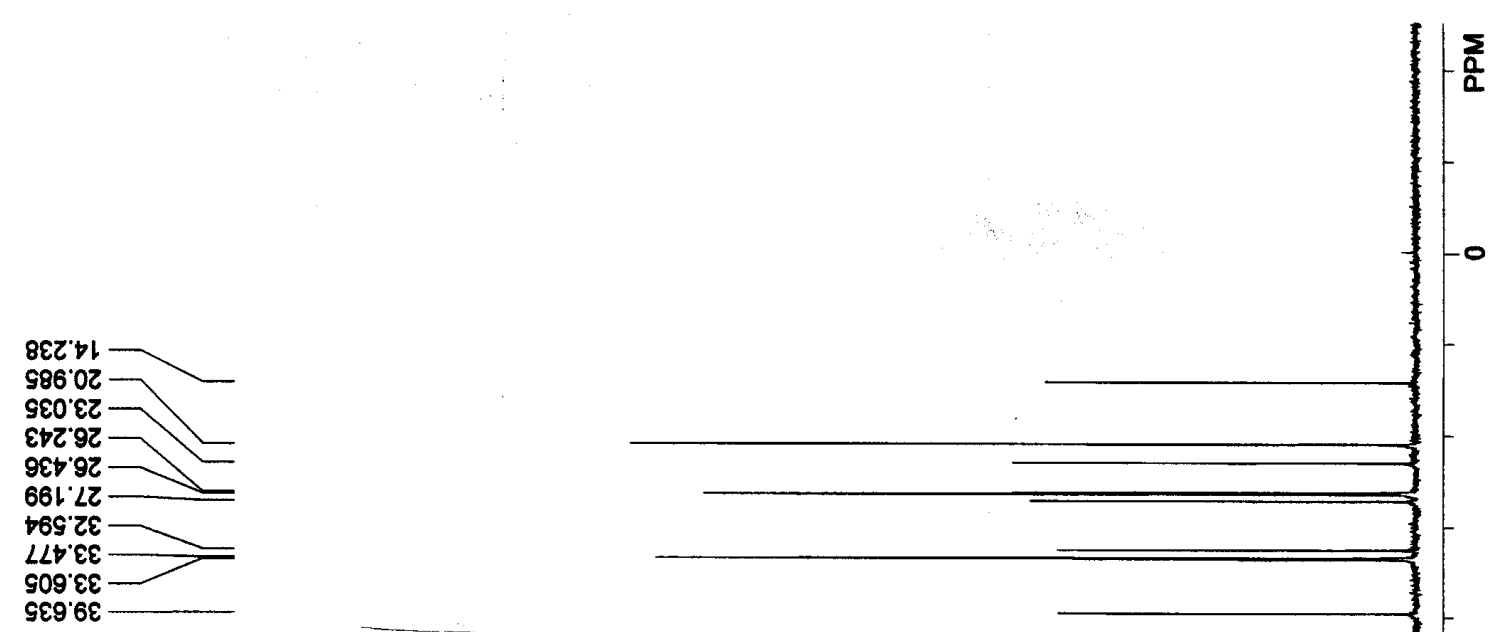

seg' 6

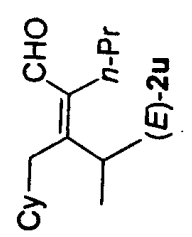

$\angle L S^{\prime} 9 L$

00022

के
\$े
爻

$\varepsilon \angle t<\varepsilon l$

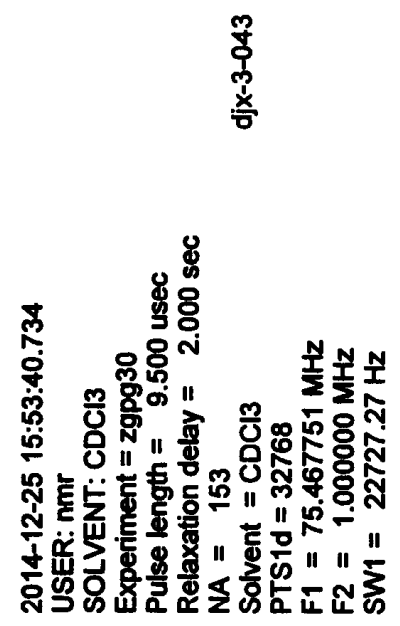

860 ' 991

osg' 261

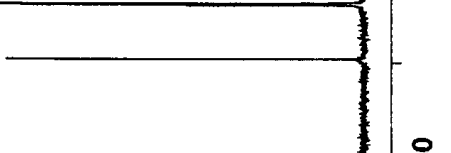



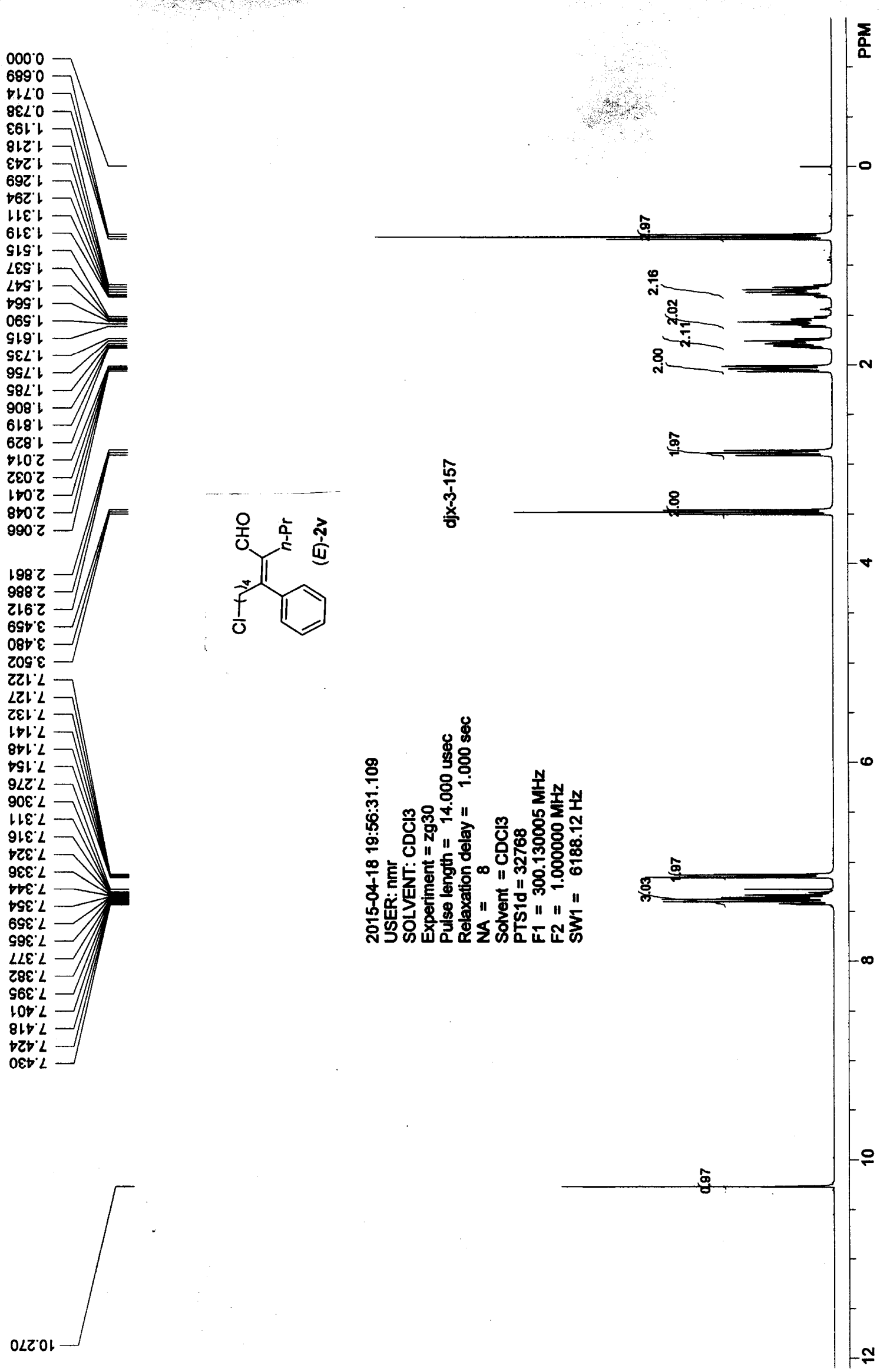
$+90 \circ b$

OZS ZZ

SOL' 92

$\angle \varepsilon 062$

IS6'L

$\varepsilon \mapsto 0 \cdot z \varepsilon$

$\llcorner\vdash \varepsilon \cdot t$

$\angle L S^{\prime} 9 L$

$000 \angle L$

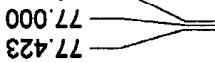

SL8'9Z

$\angle t 9^{\circ} \angle Z$

$06 Z^{\prime} 821$

$9 \succ \varepsilon \cdot 8 \varepsilon \downarrow$

Ot। Lt L

09t.695

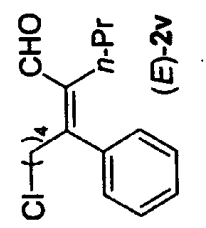

899' 61

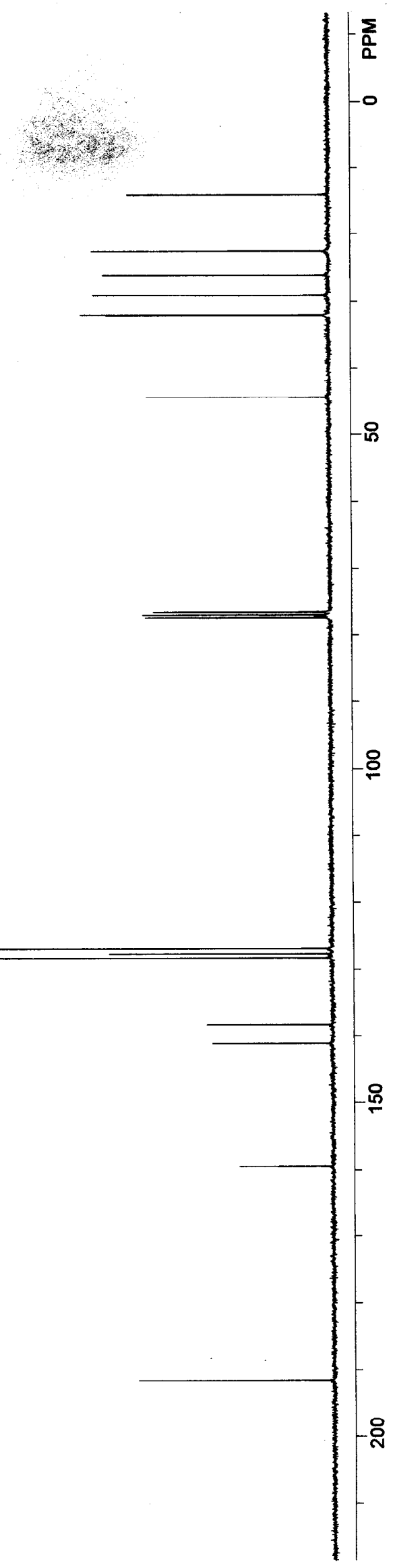



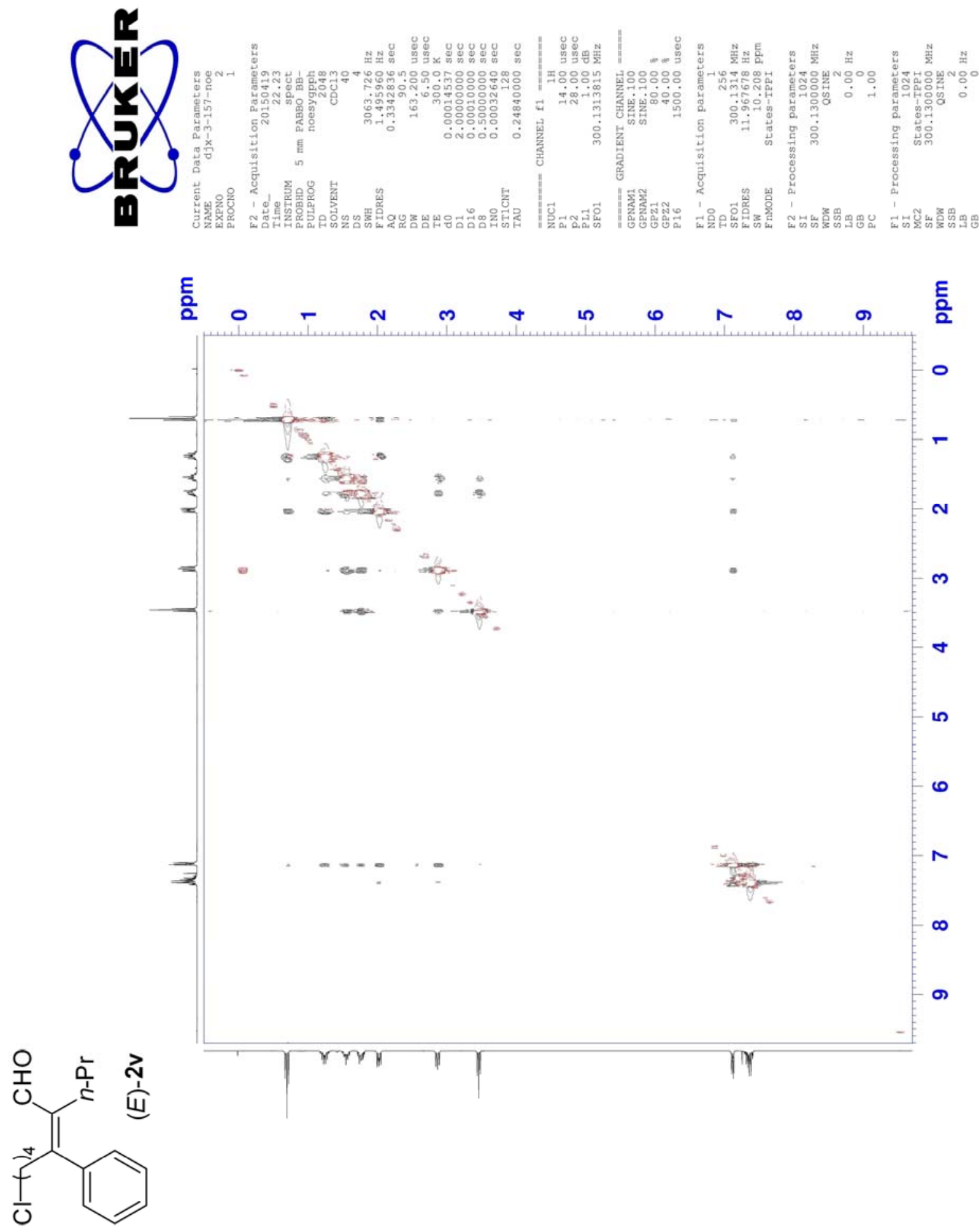

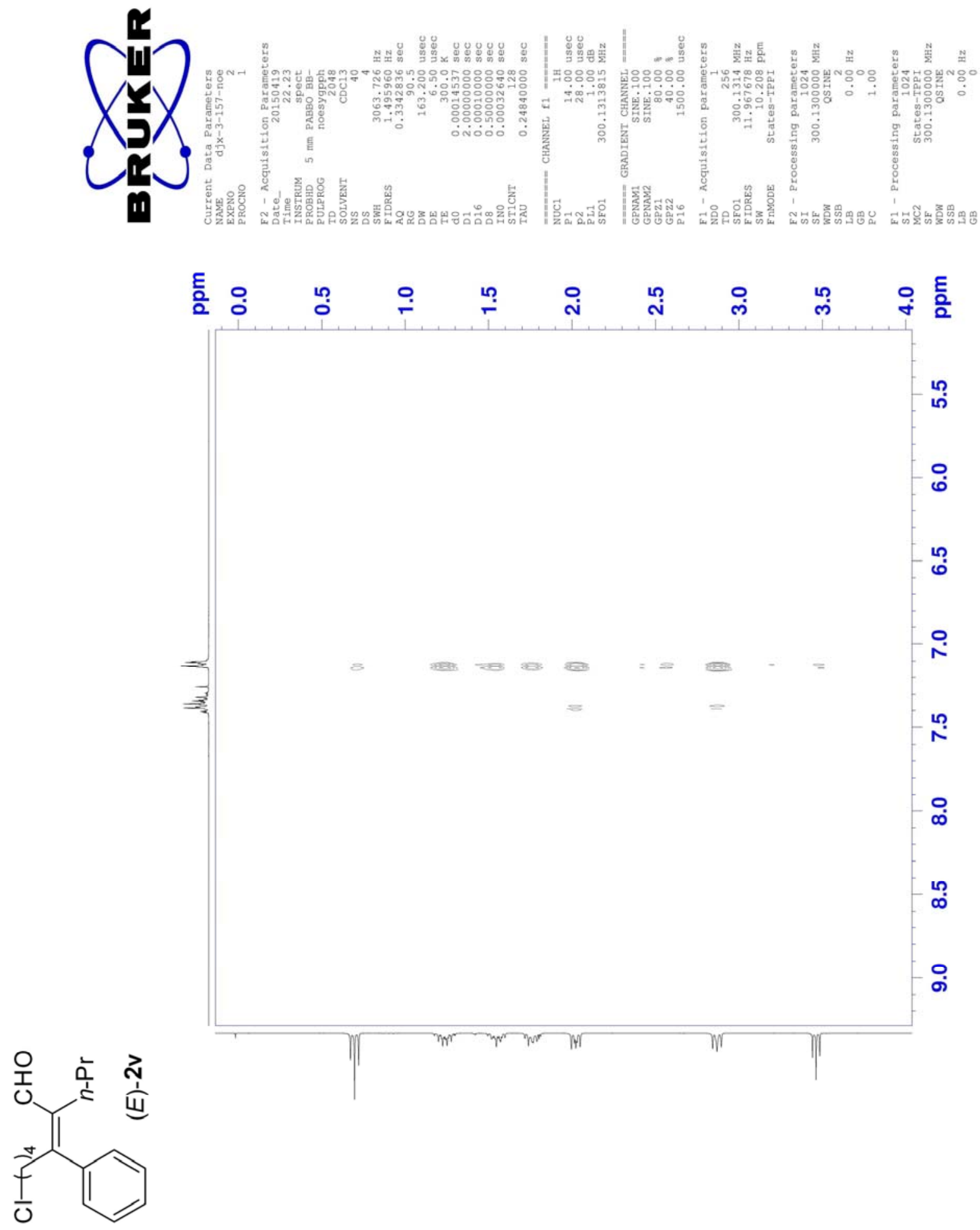

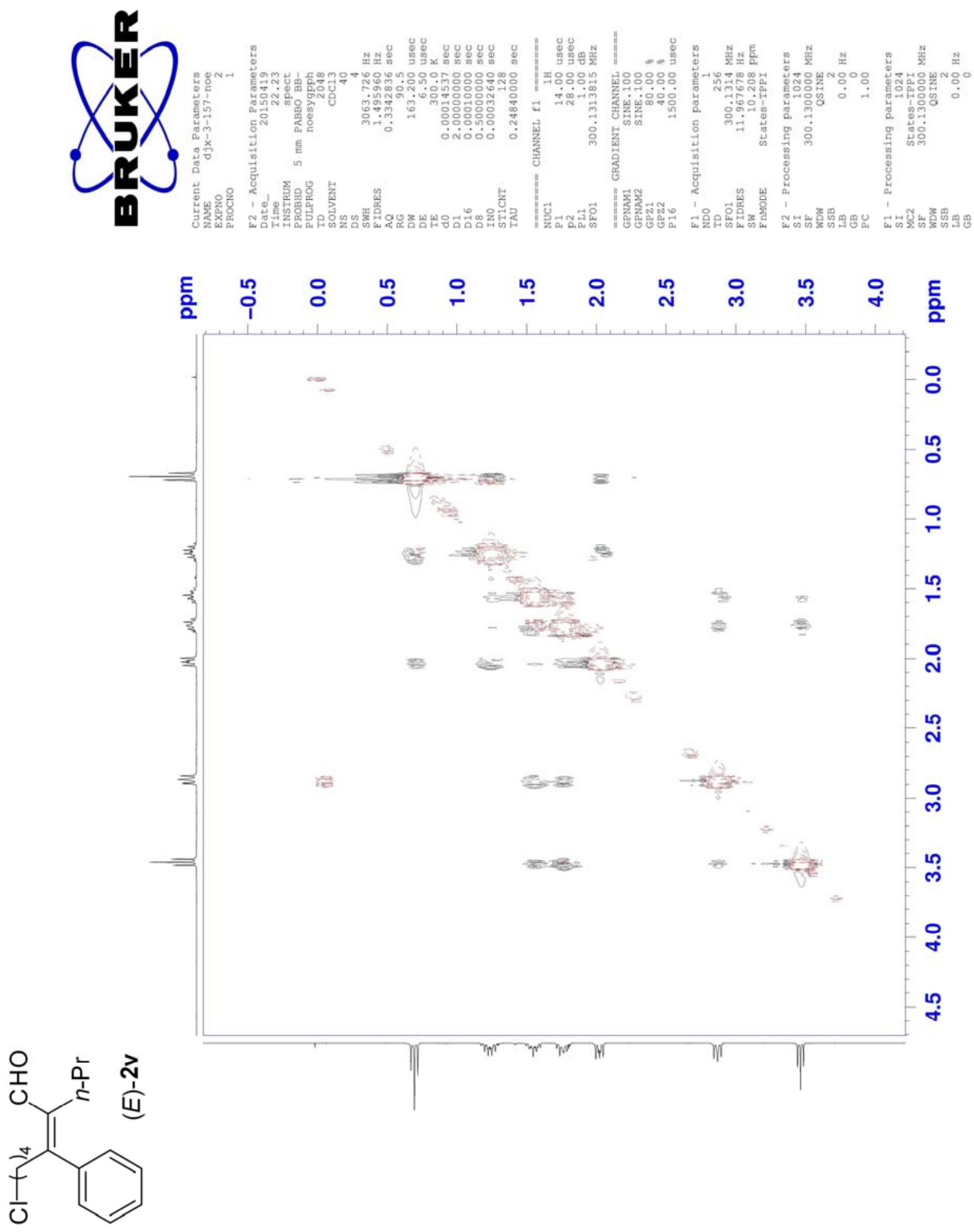

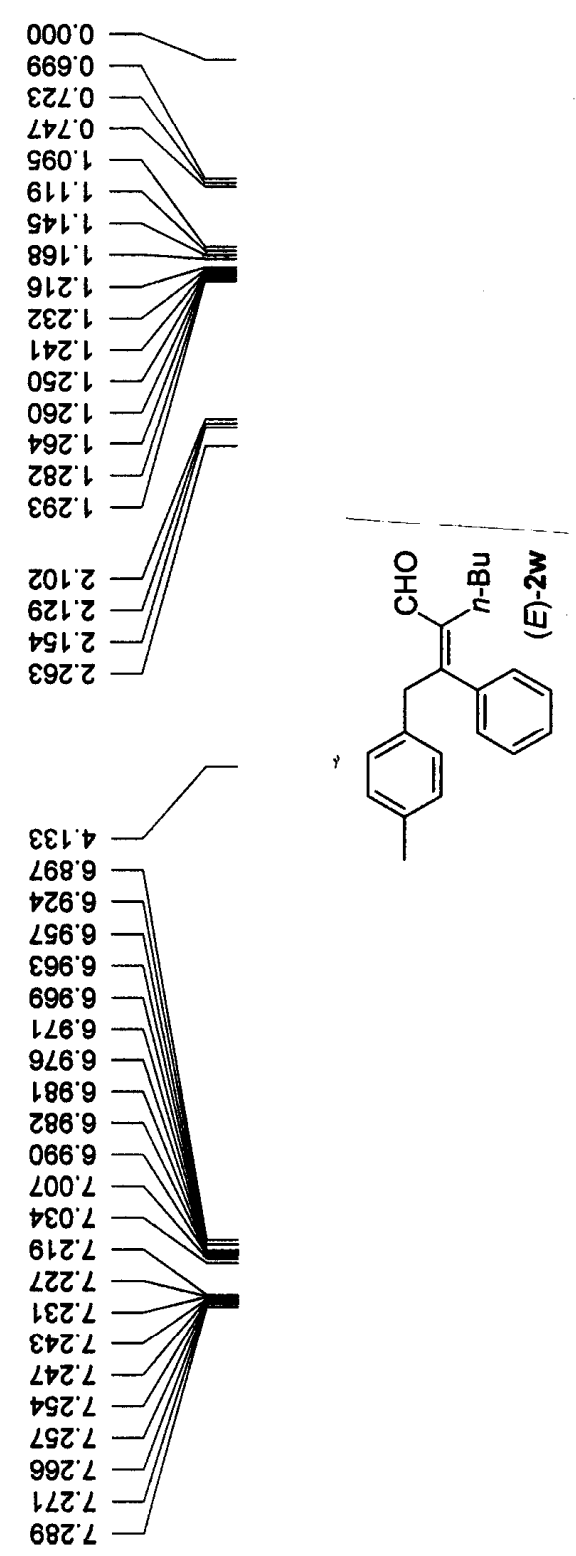

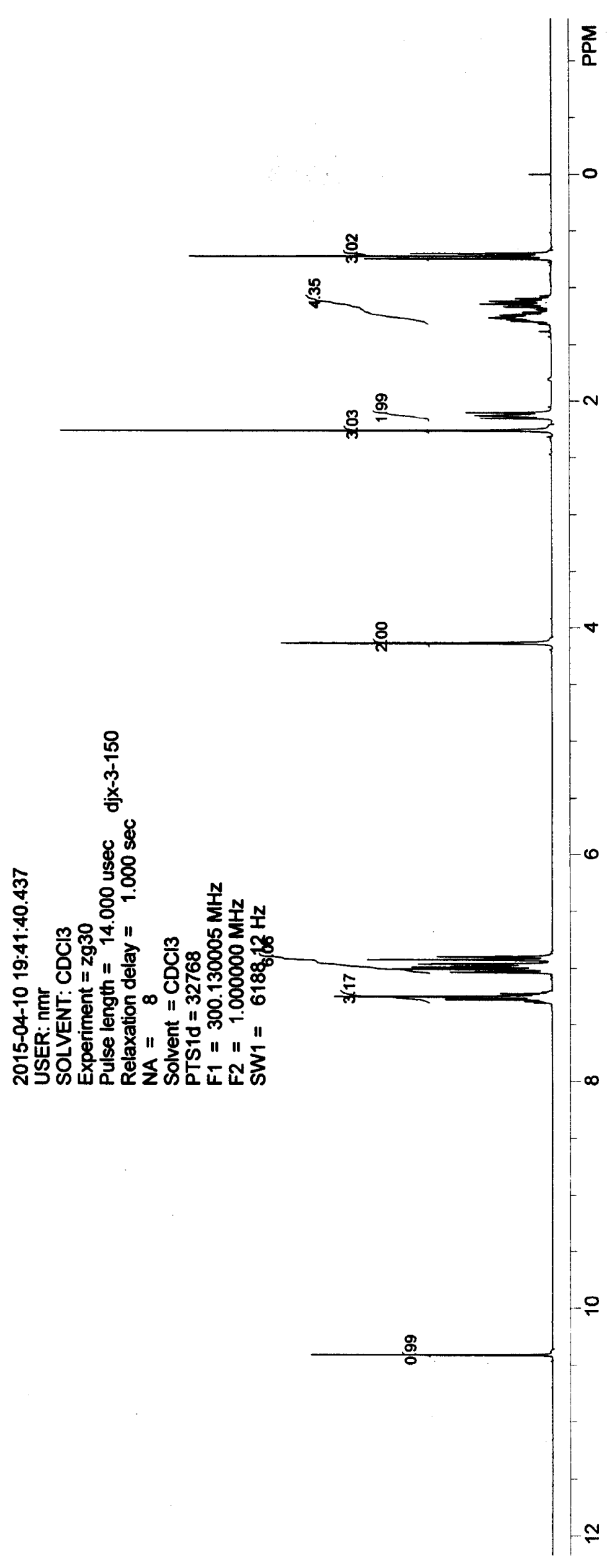



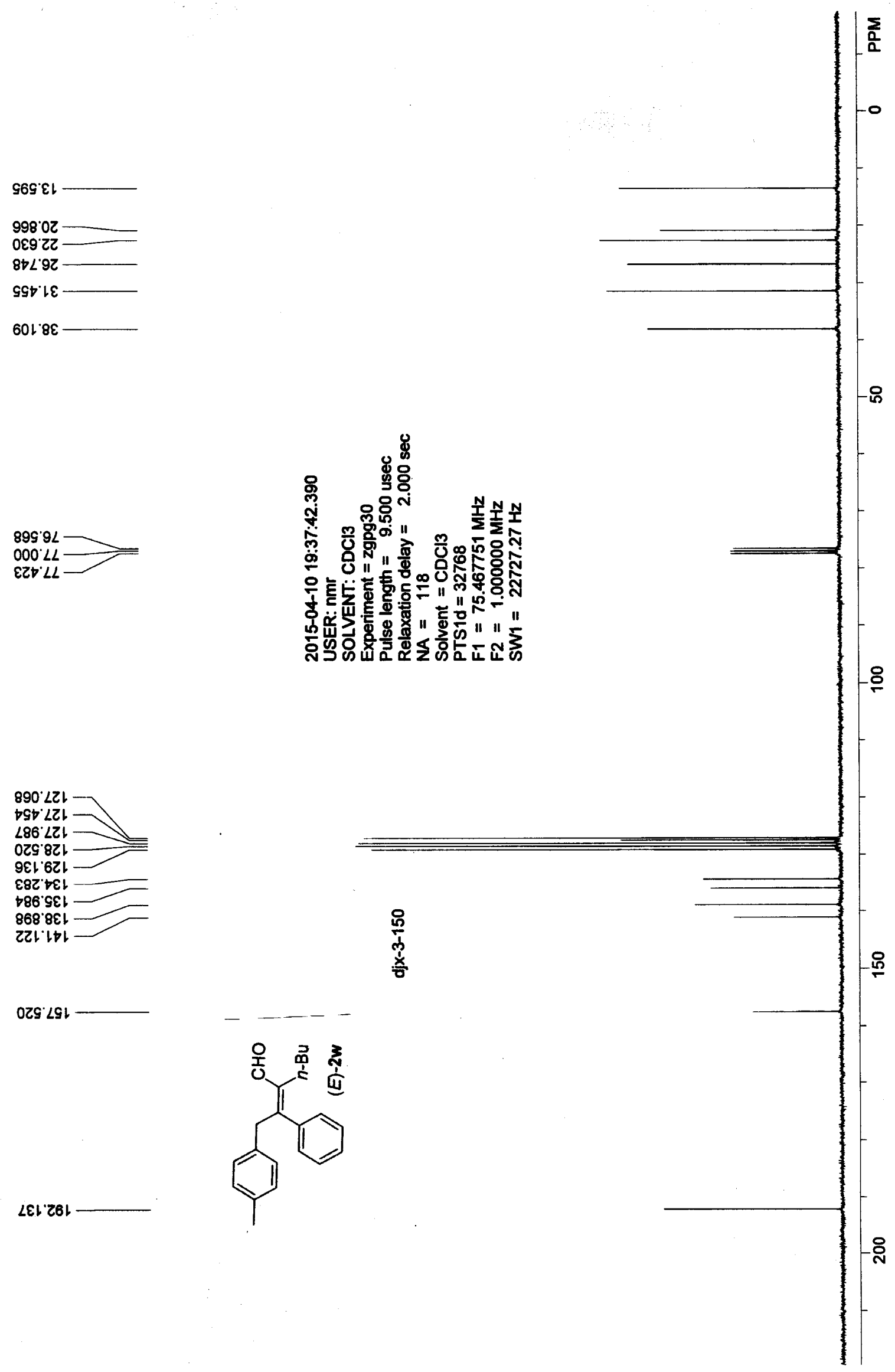

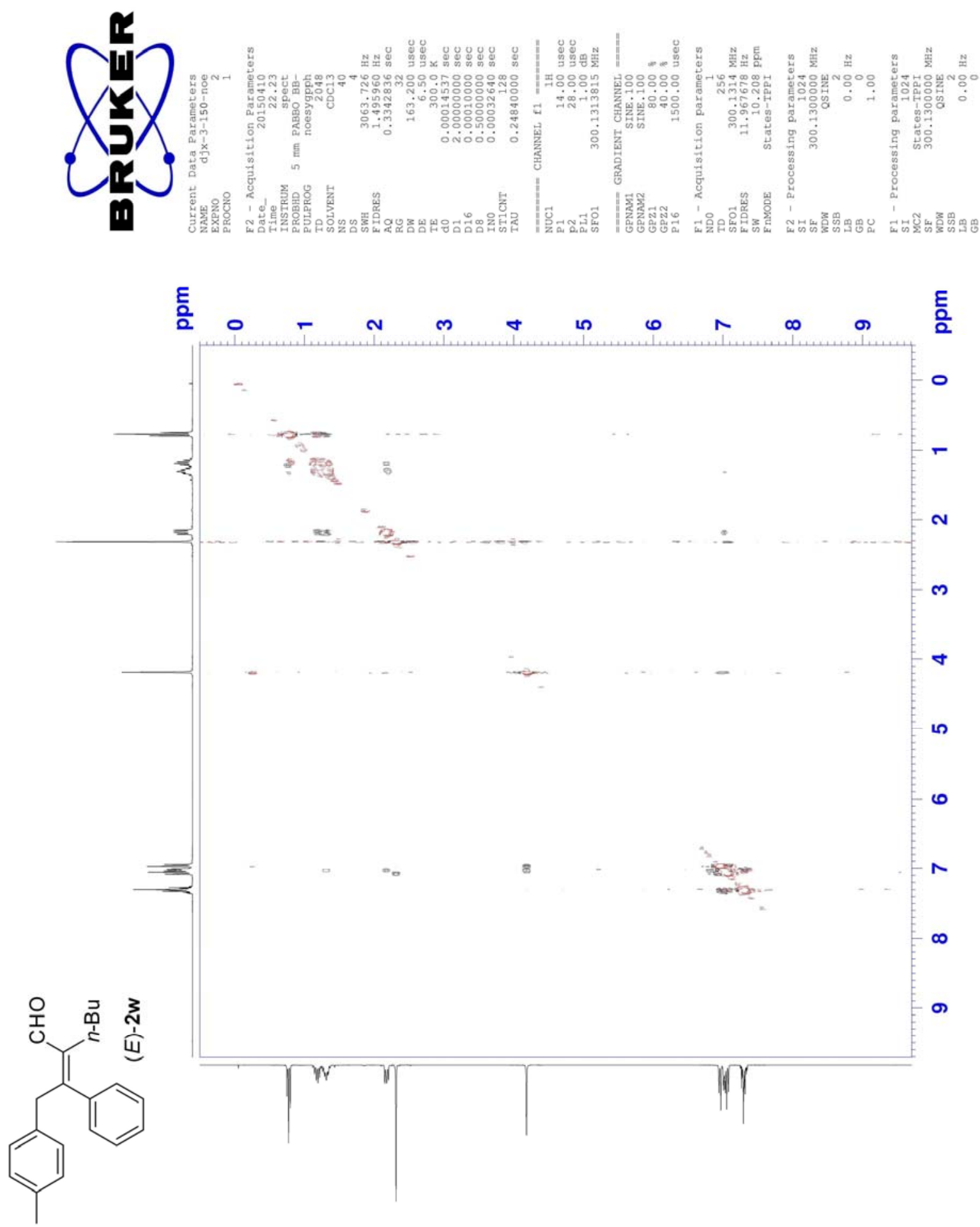

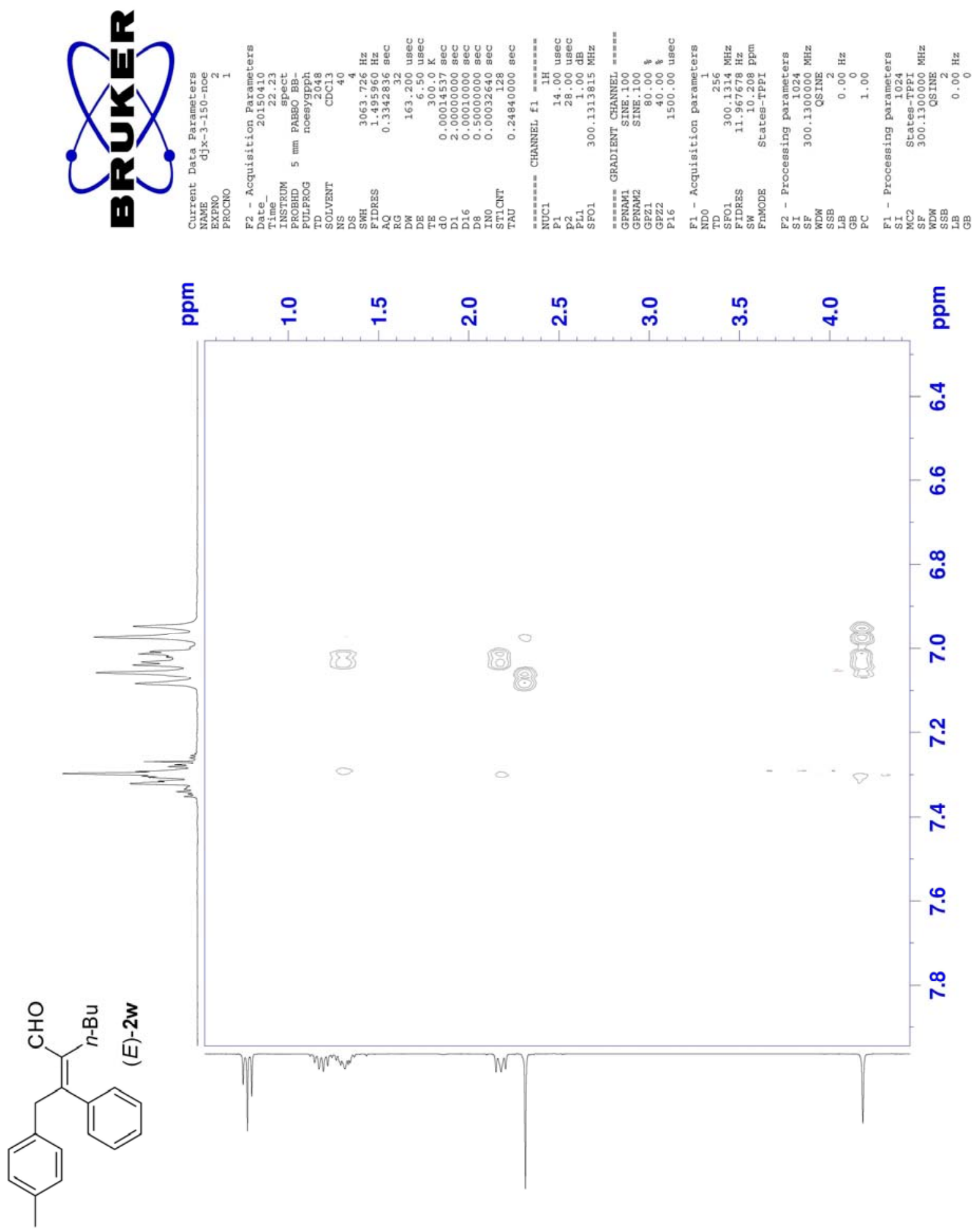

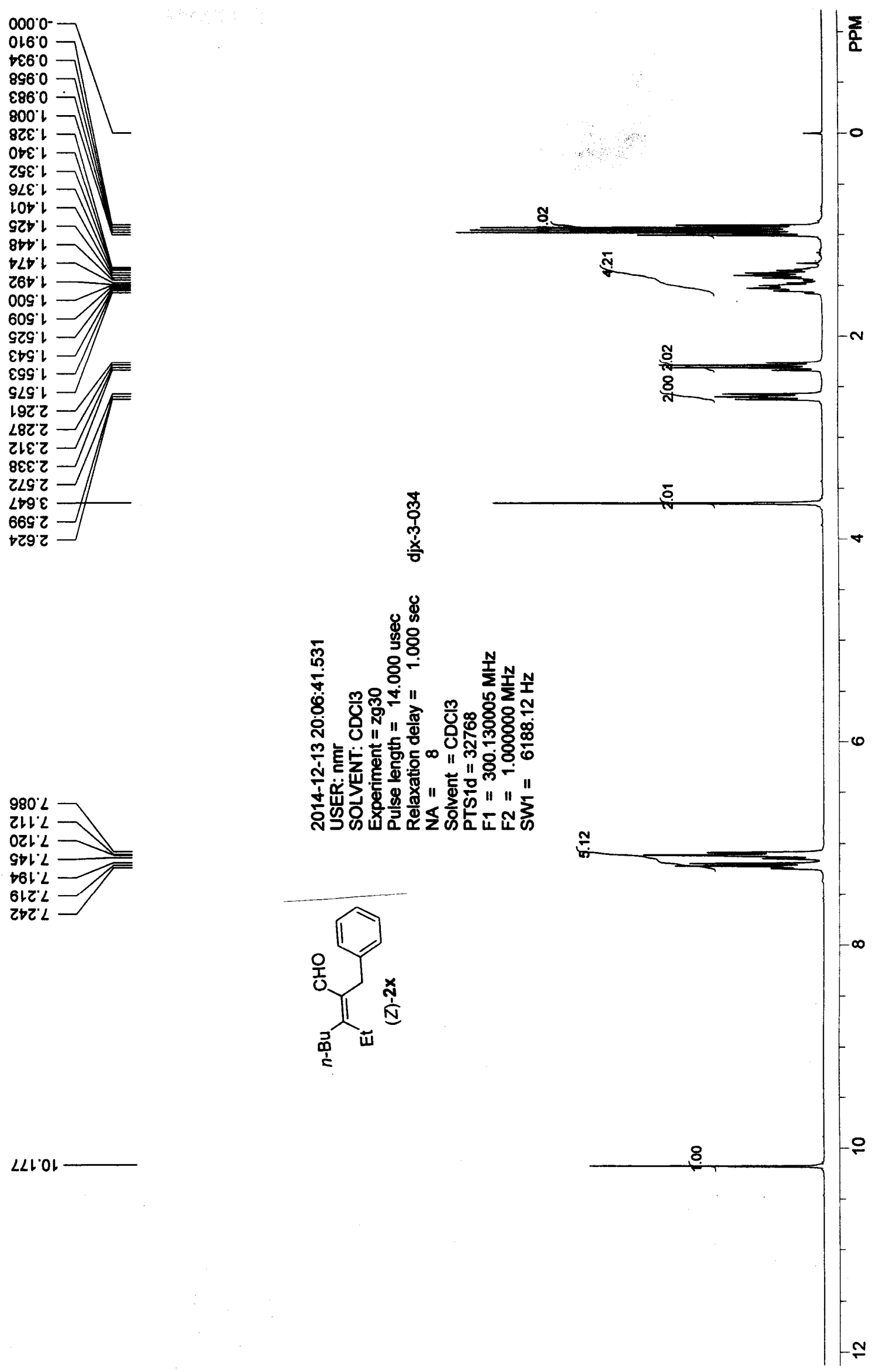

$\angle L L^{\circ} \mathrm{OL}$ 
$\angle 80$ '

EEL $E$ L

$89 L 2 Z$

$\checkmark 7082$

$\angle \forall 6.62$

98. $0 E$
L $\angle 6^{\circ} Z E$

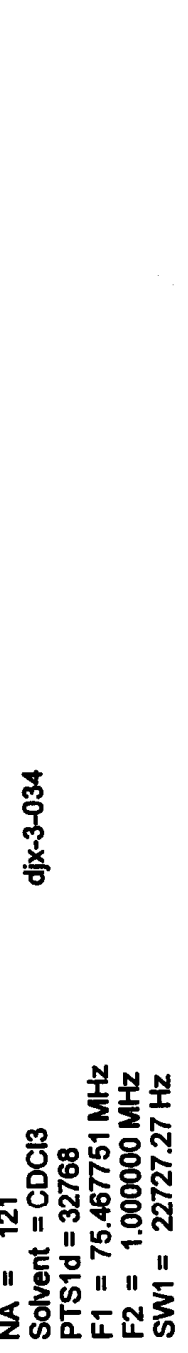

LLG'OL

$8 เ t^{\prime} 99 t$

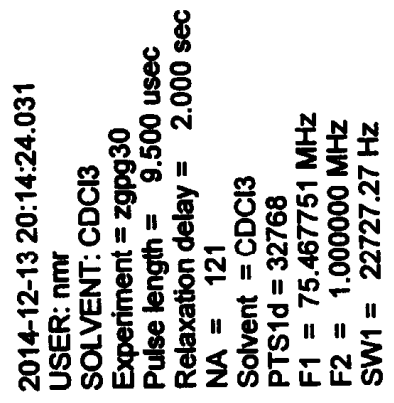

889 'cZl

$6 \vdash 8$ 'LL

Z9. 821

08Lt大El

clcobl

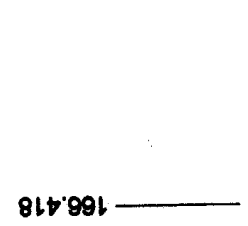

$921 \cdot 161$

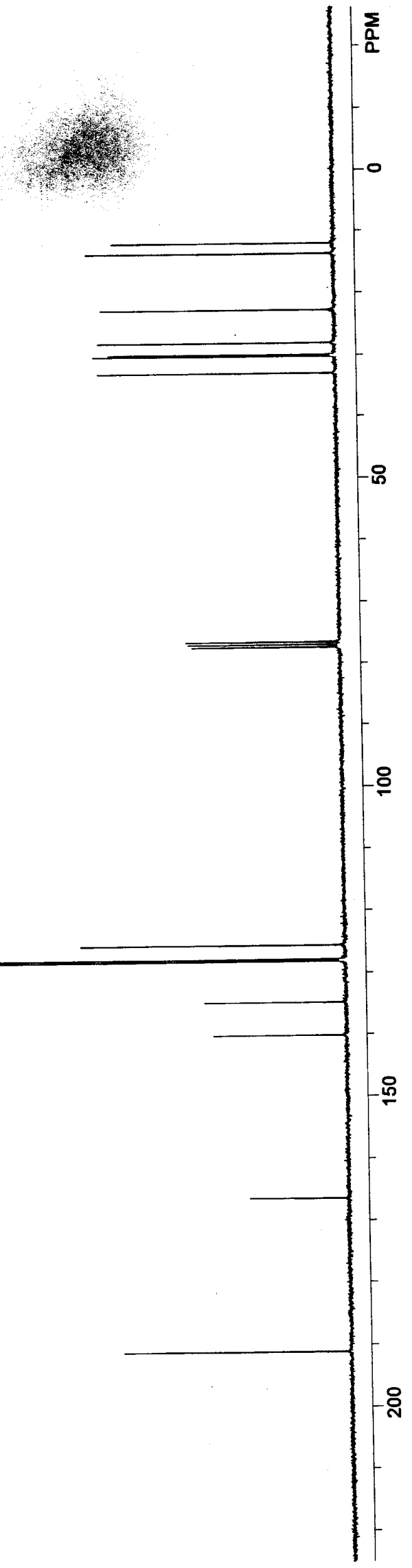



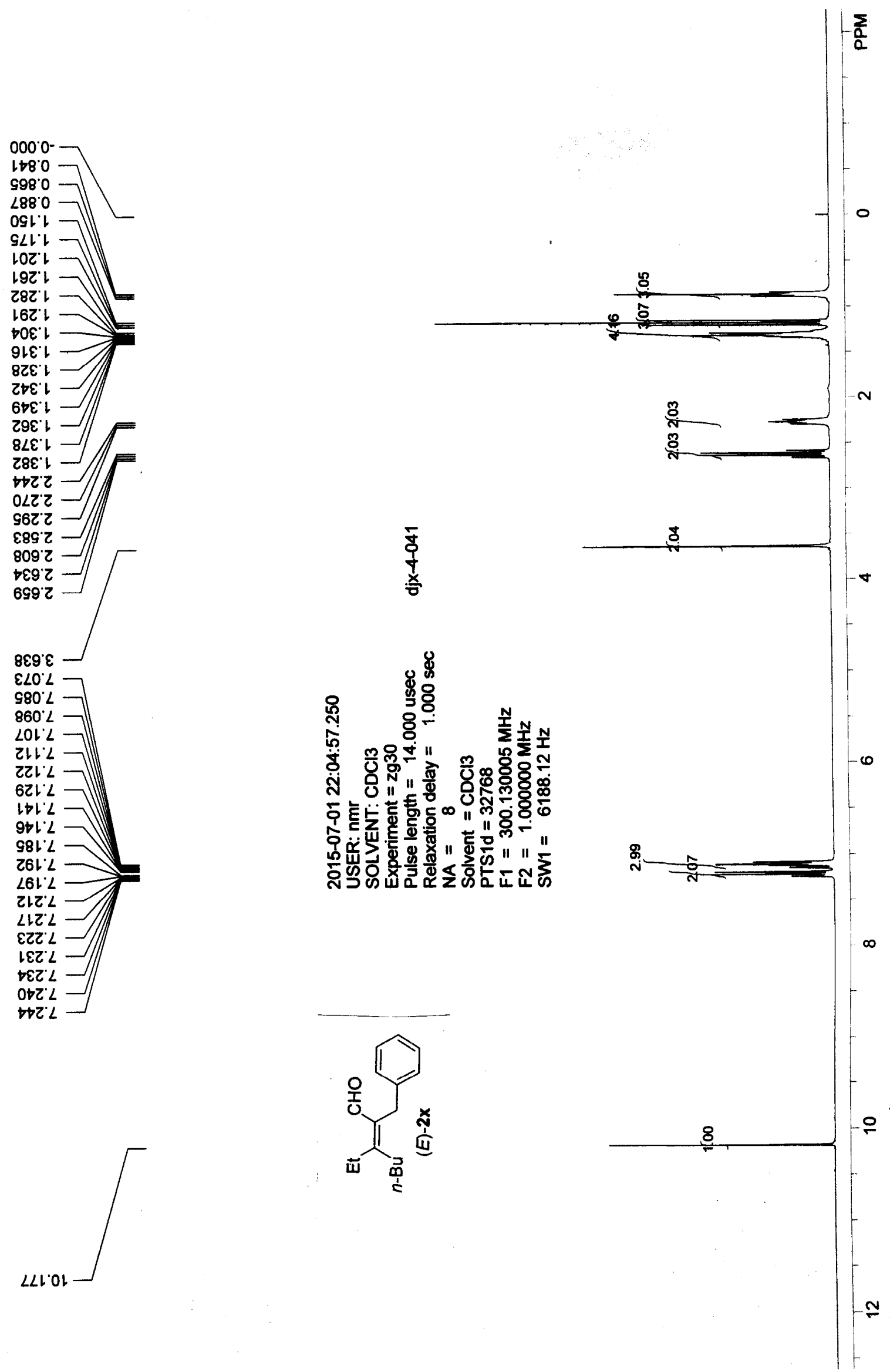


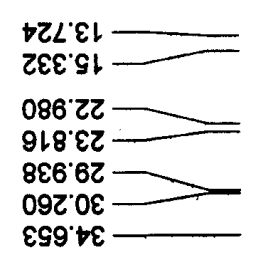

LLS' $9 L$

$000^{\circ} \angle L$<smiles>[CH]/C(OC)=C(/[O-])Cc1ccccc1</smiles>

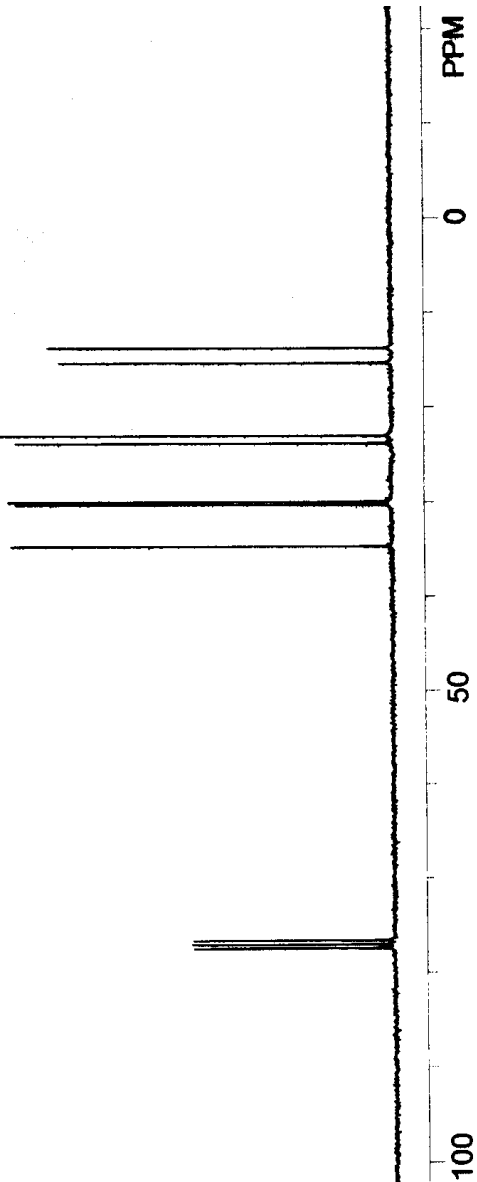

6LG'SZI

$6 \succ 8^{\circ} \angle Z L$

$\downarrow \varepsilon$ เ 82 L

$\varepsilon \varepsilon 9^{\prime} \downarrow \varepsilon$

9LZ OtL

$\frac{7}{0}$
$\dot{y}$
$\frac{x}{0}$

ষ্్ㅇ용

ชั

ธ

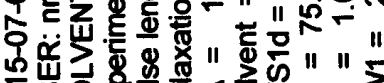

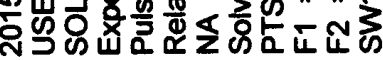

$+69^{\prime} 991$

$\downarrow \varepsilon 0.161$ 

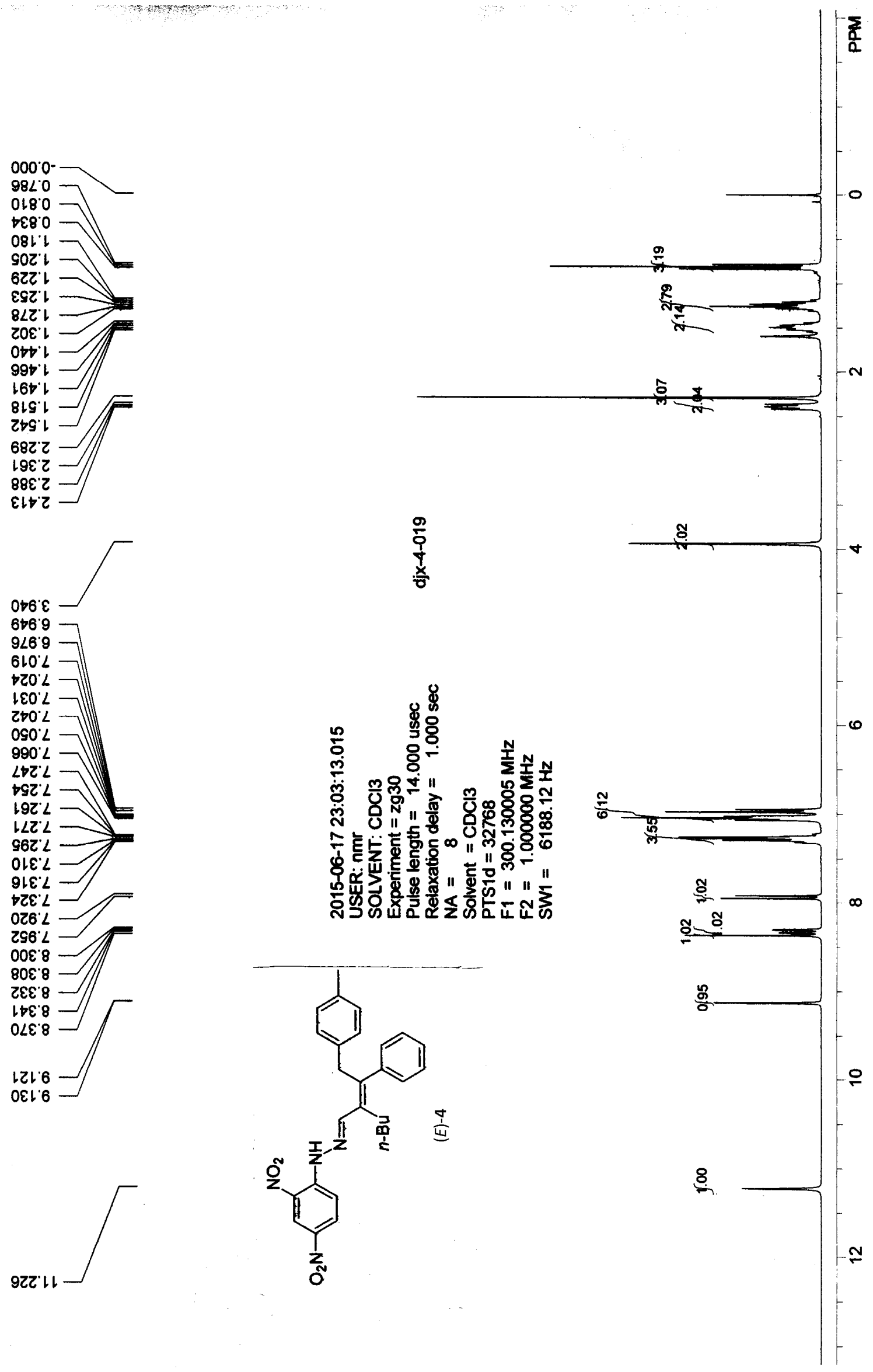


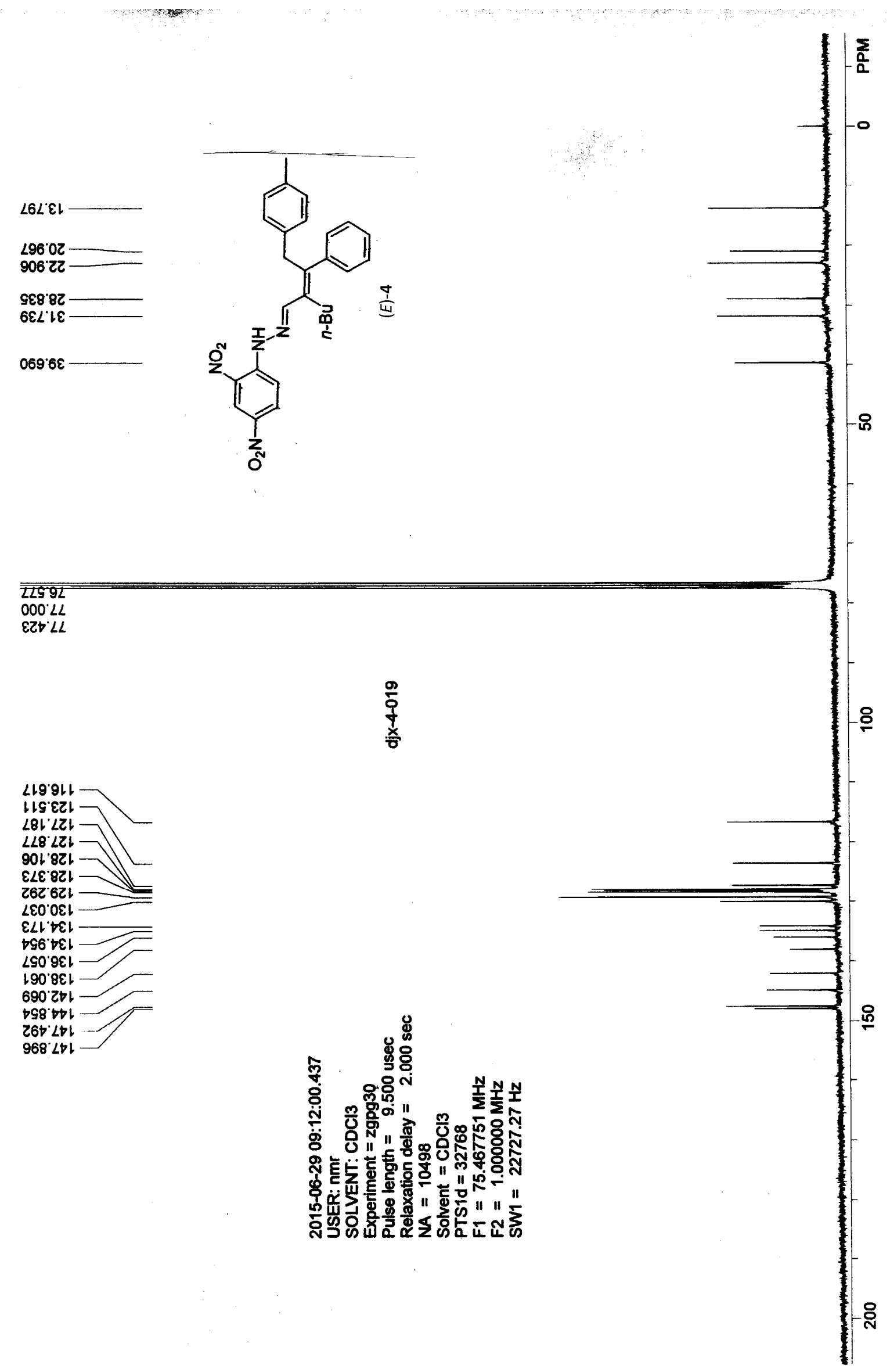



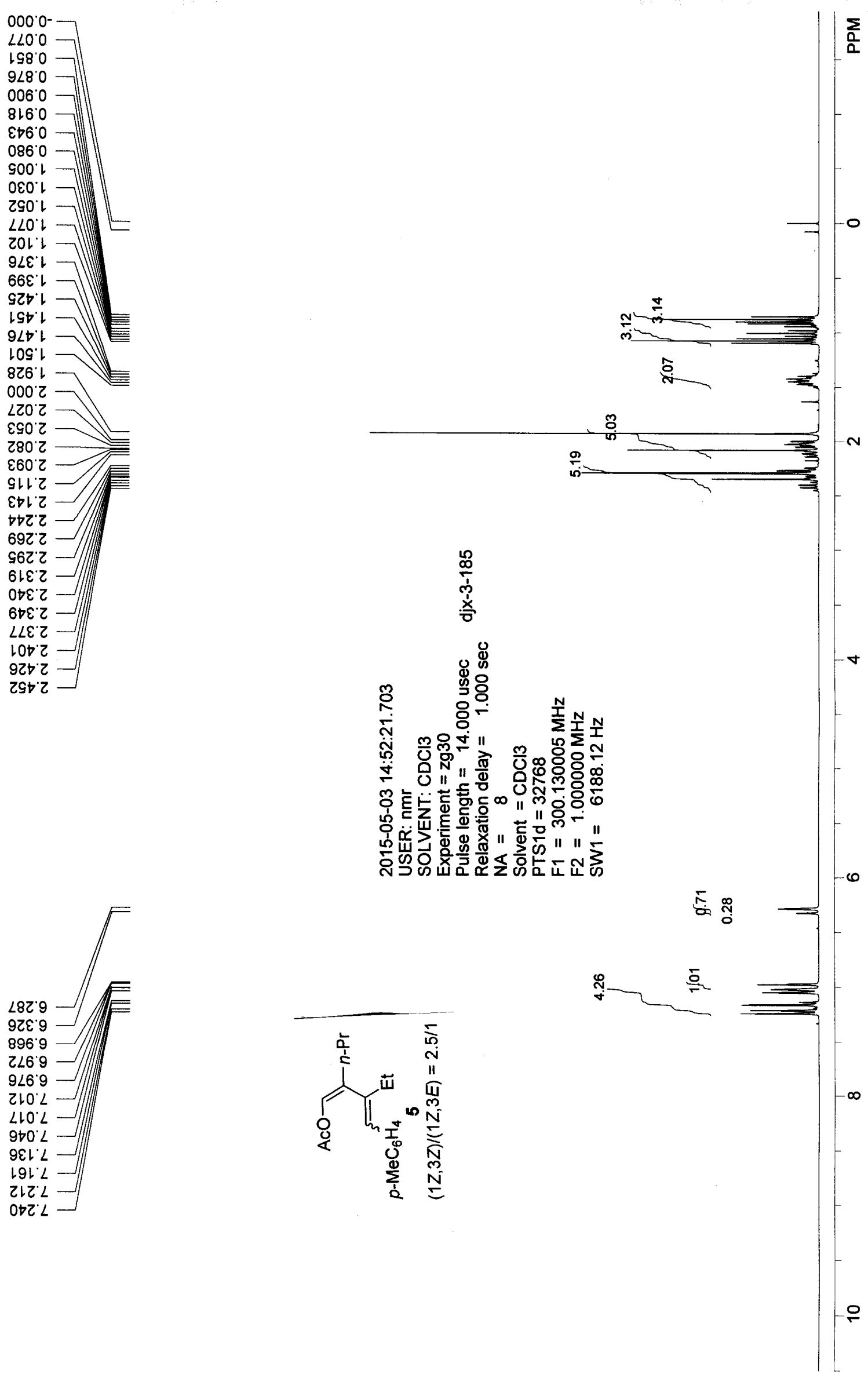

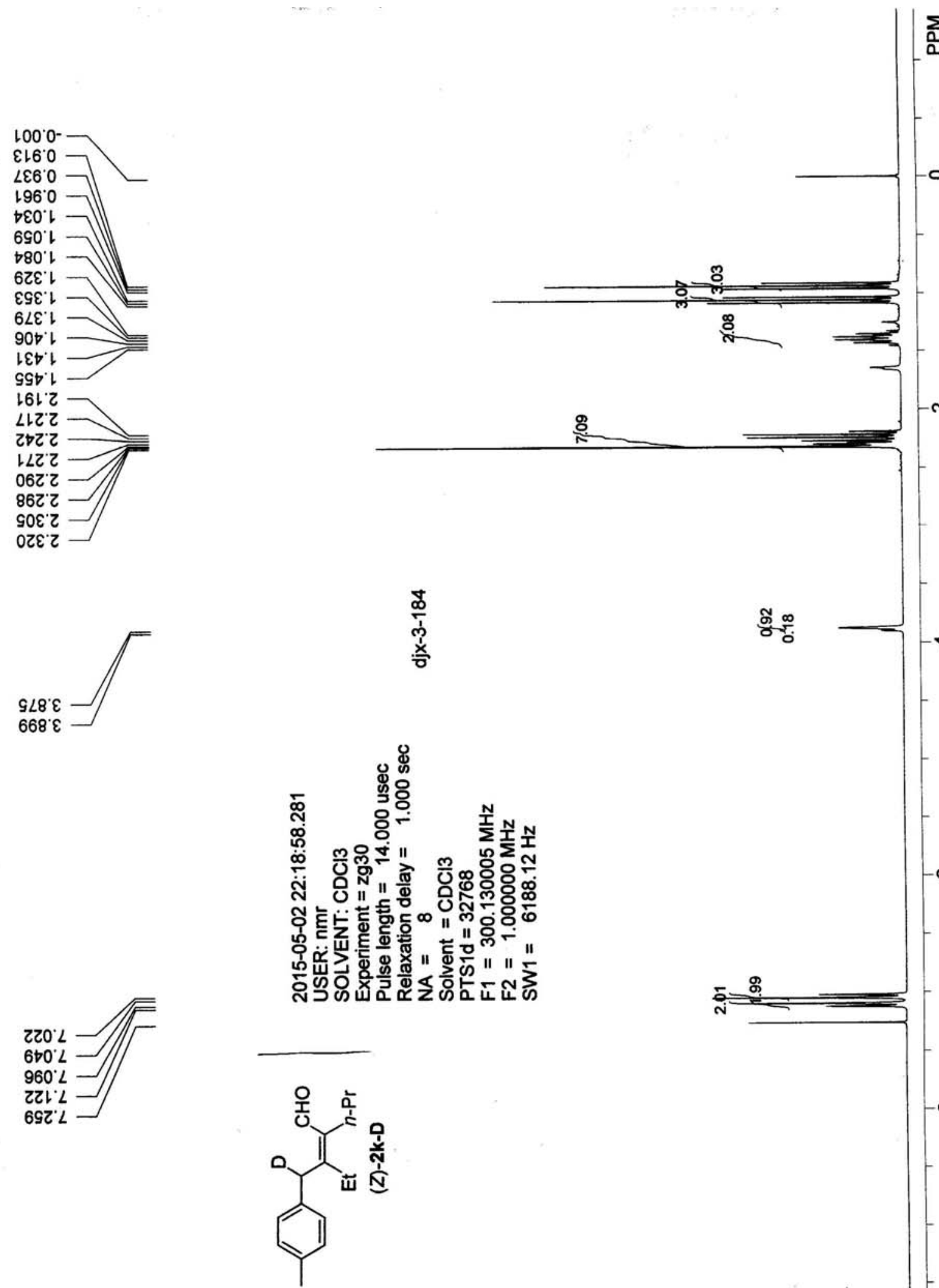

$281^{\circ} \mathrm{OL}$

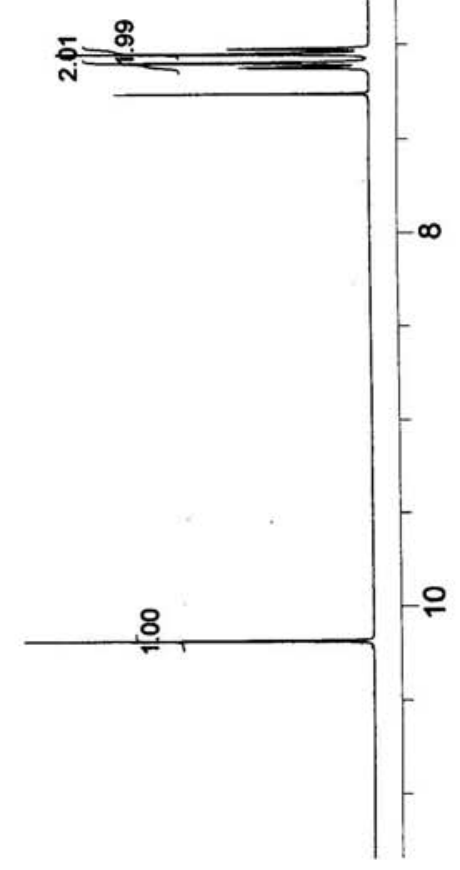




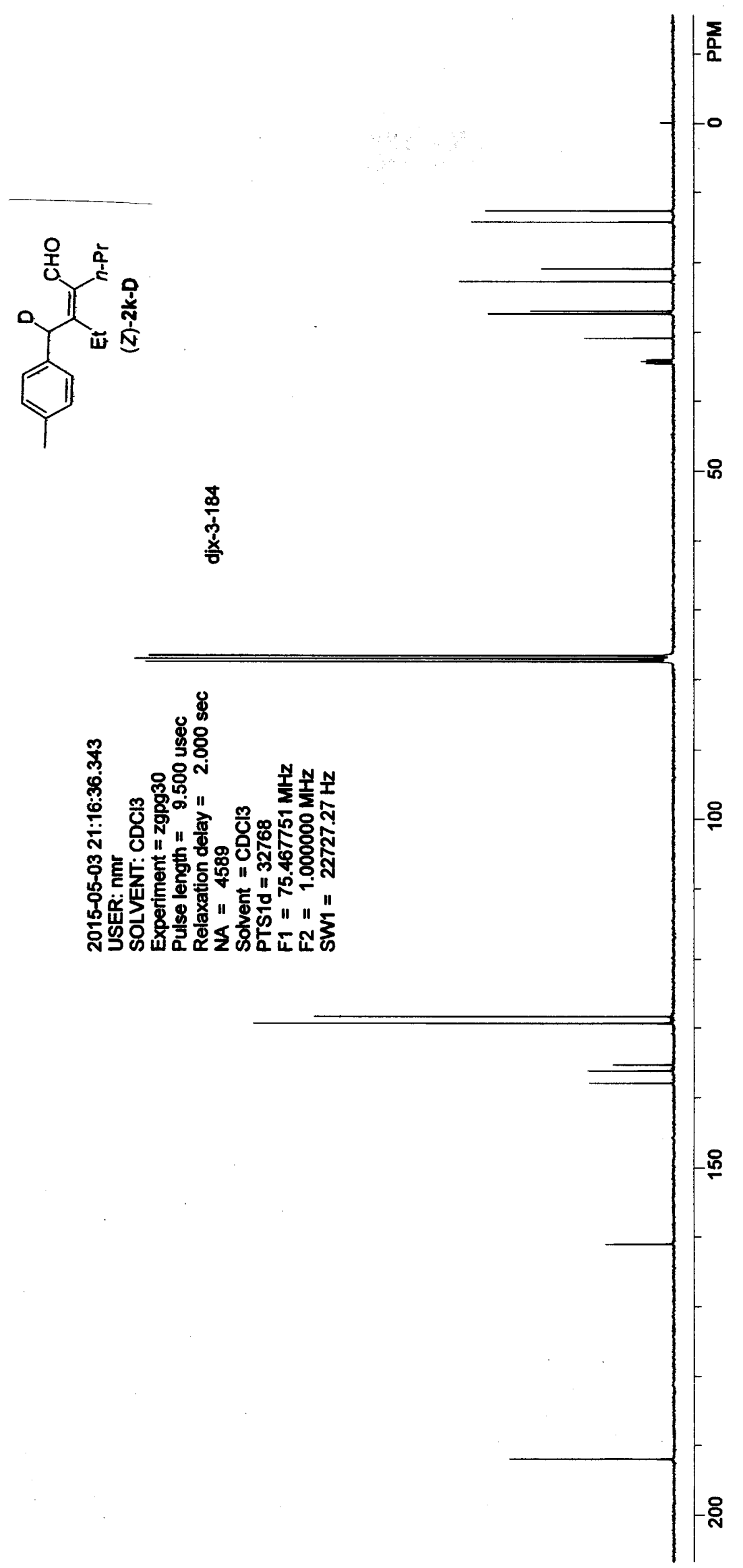

$000 \%$ $\varepsilon Z \downarrow \angle L$

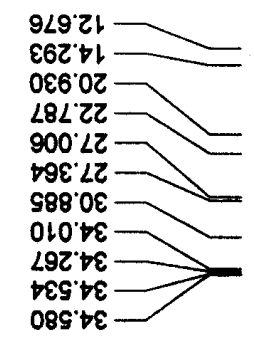

998 82

L४ع 62 L

0 te's

$961 \cdot 9 \varepsilon 1$

$2+6 . \angle \varepsilon$

$\angle 80^{\circ}$ L9

$\angle 10^{\circ} \mathrm{Z} 6 \mathrm{~L}$

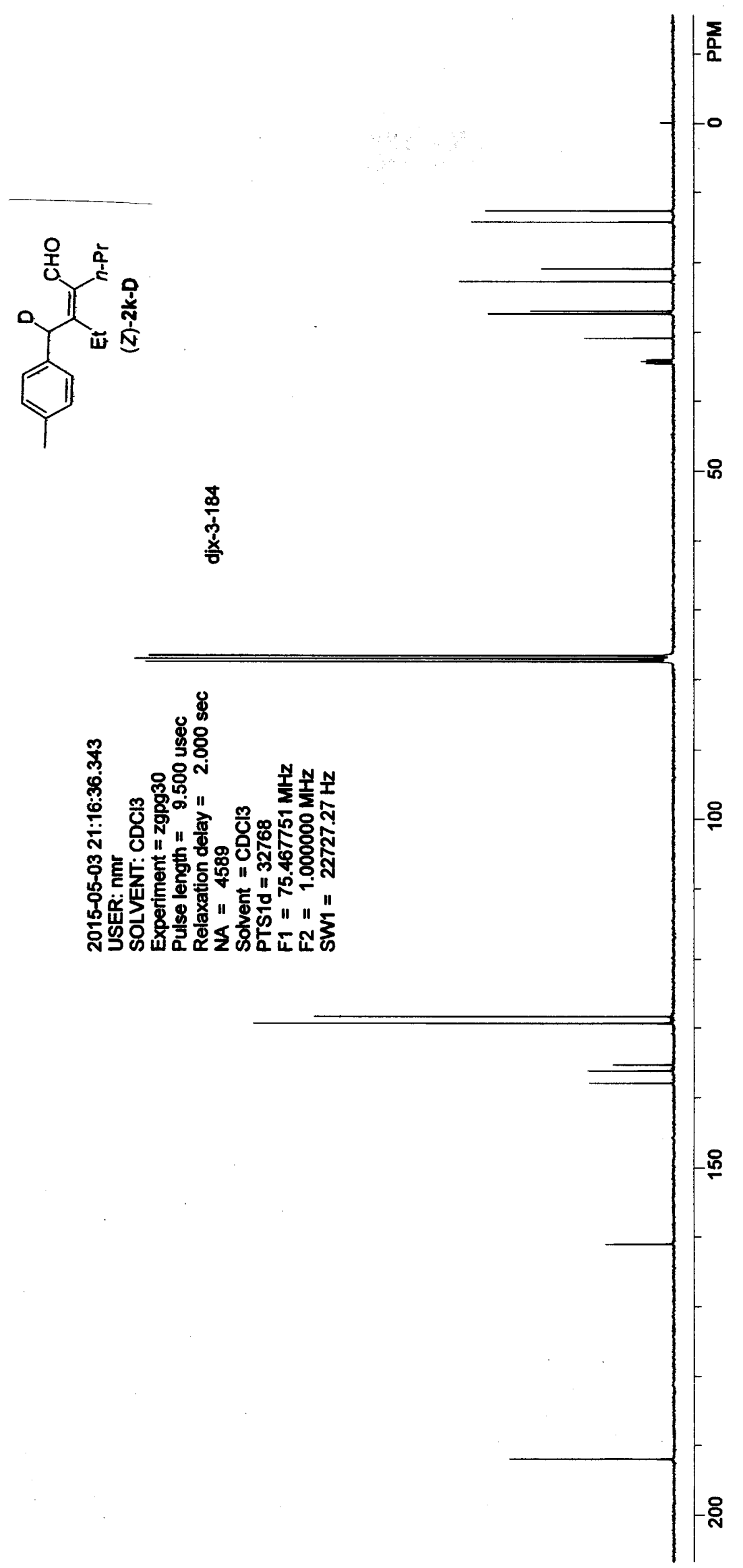



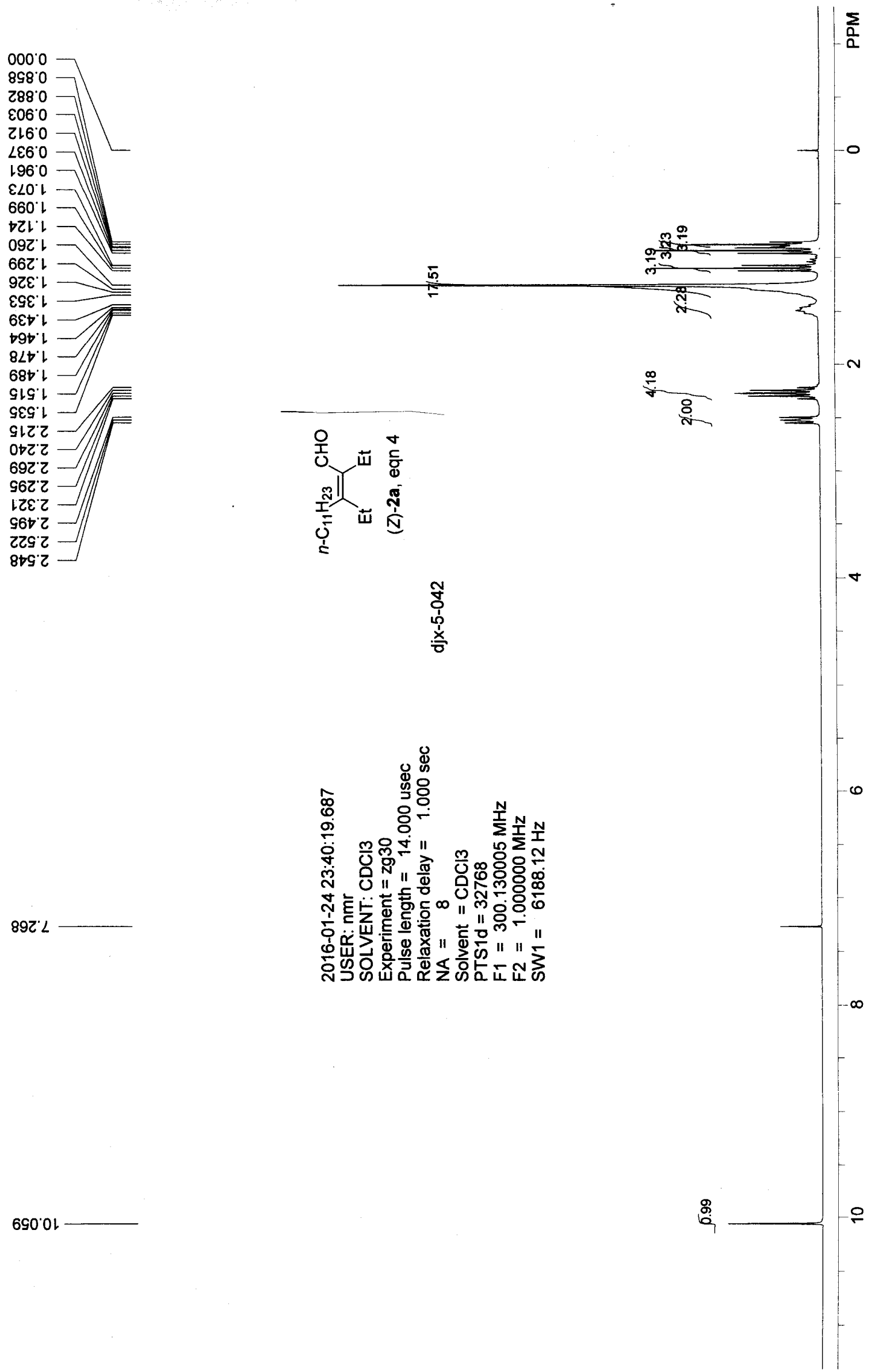
LLE 81

$99^{\circ} \mathrm{ZZ}$

$68 t^{\prime} L Z$

O०६'6Z

$\varepsilon 8 \varepsilon^{\circ} 6 \mathrm{Z}$

ZOS' 62

$9 \angle 962$

S69.6Z

$16 \mathrm{~L}^{\circ} 0 \mathrm{E}$

ZL8'OE

$\varepsilon 88^{\circ}\llcorner\varepsilon$

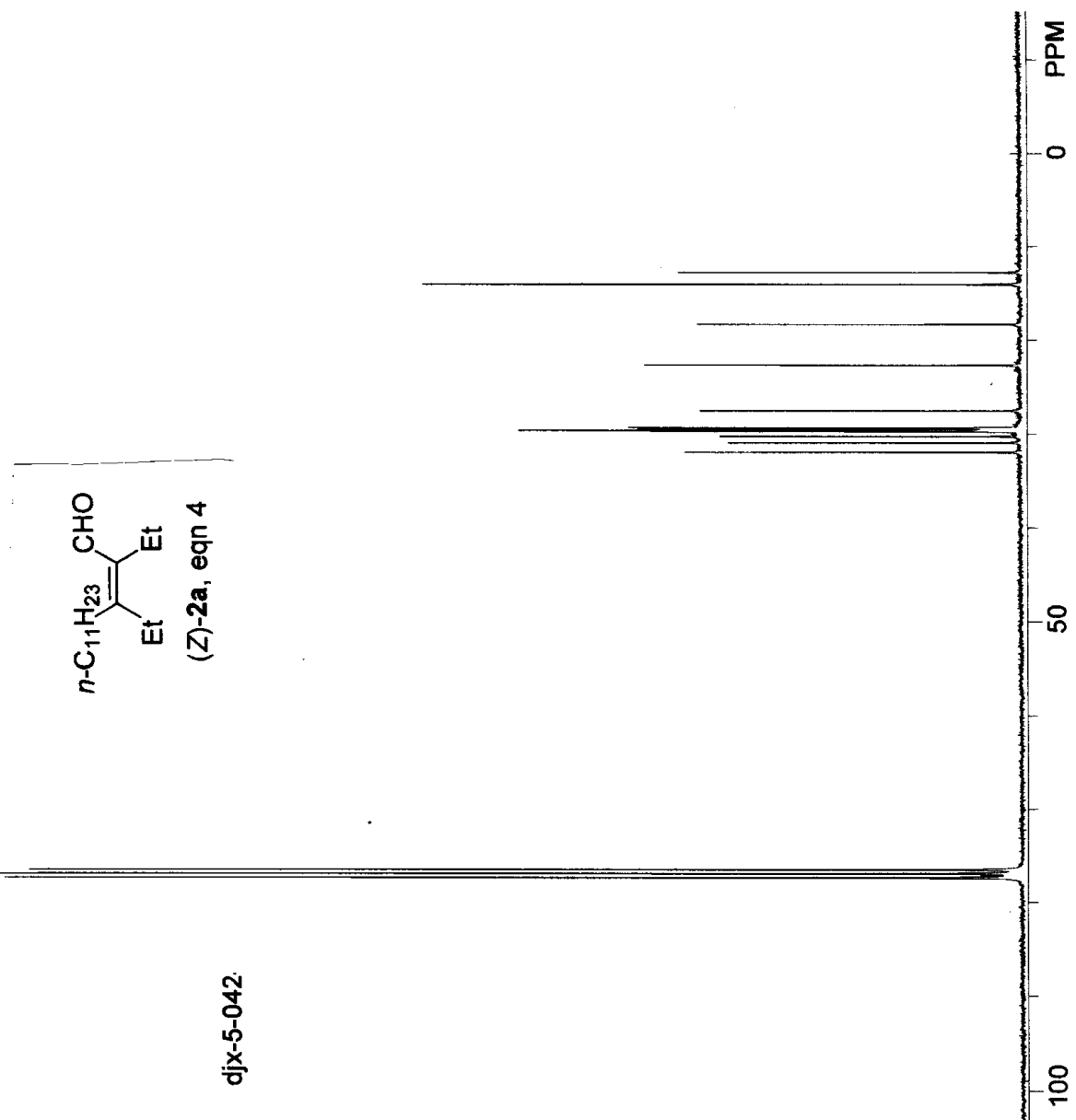

$8 \varepsilon 6^{\circ} L \varepsilon\llcorner$

$900^{\circ} L L$

$8 Z 7 L L$

totit $t 9 t$

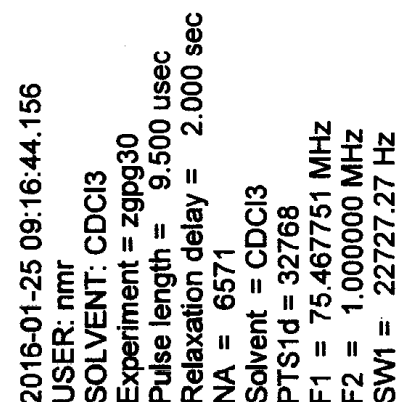

LtS'L6L 

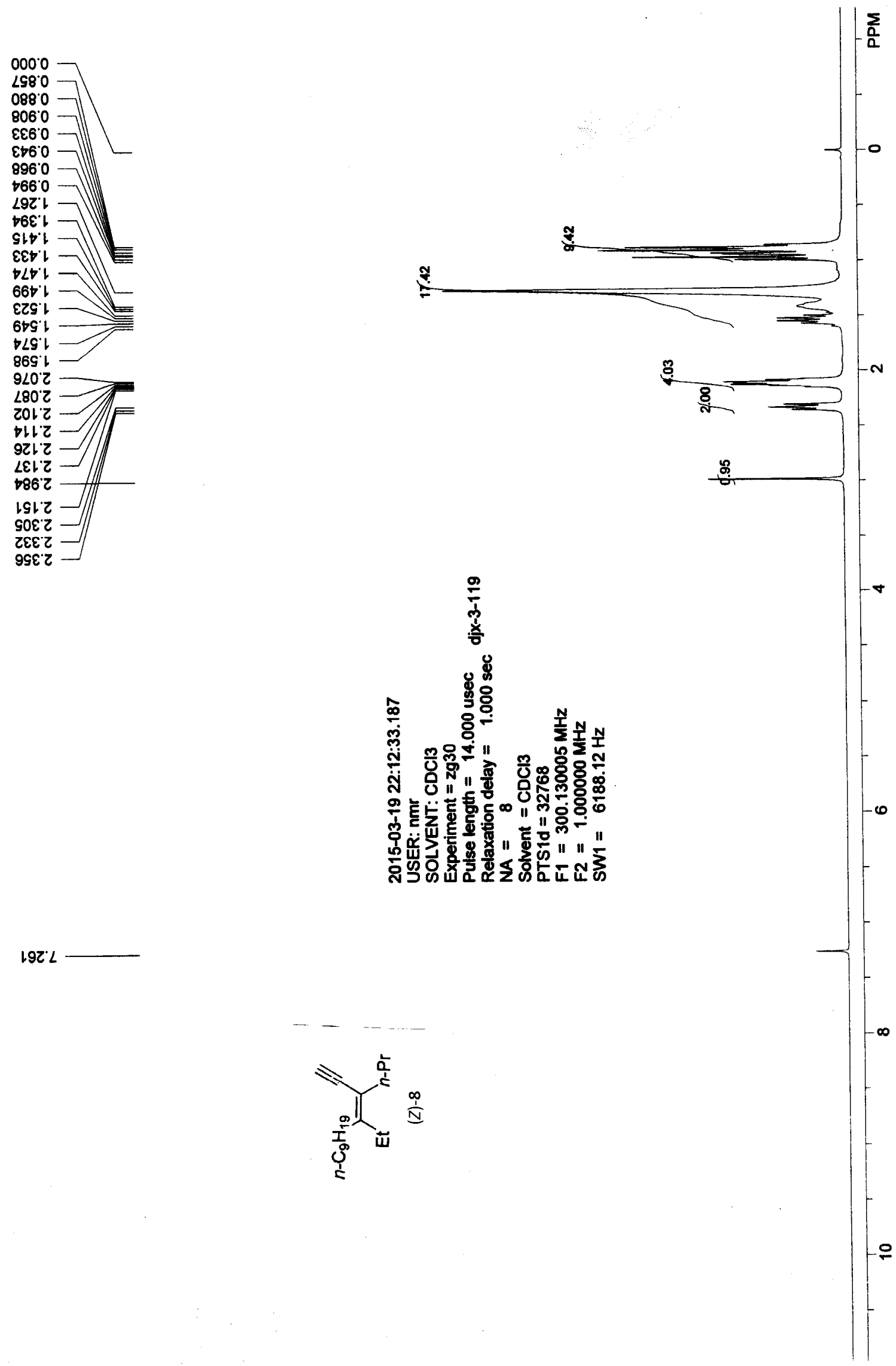

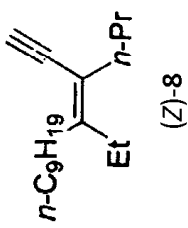

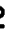



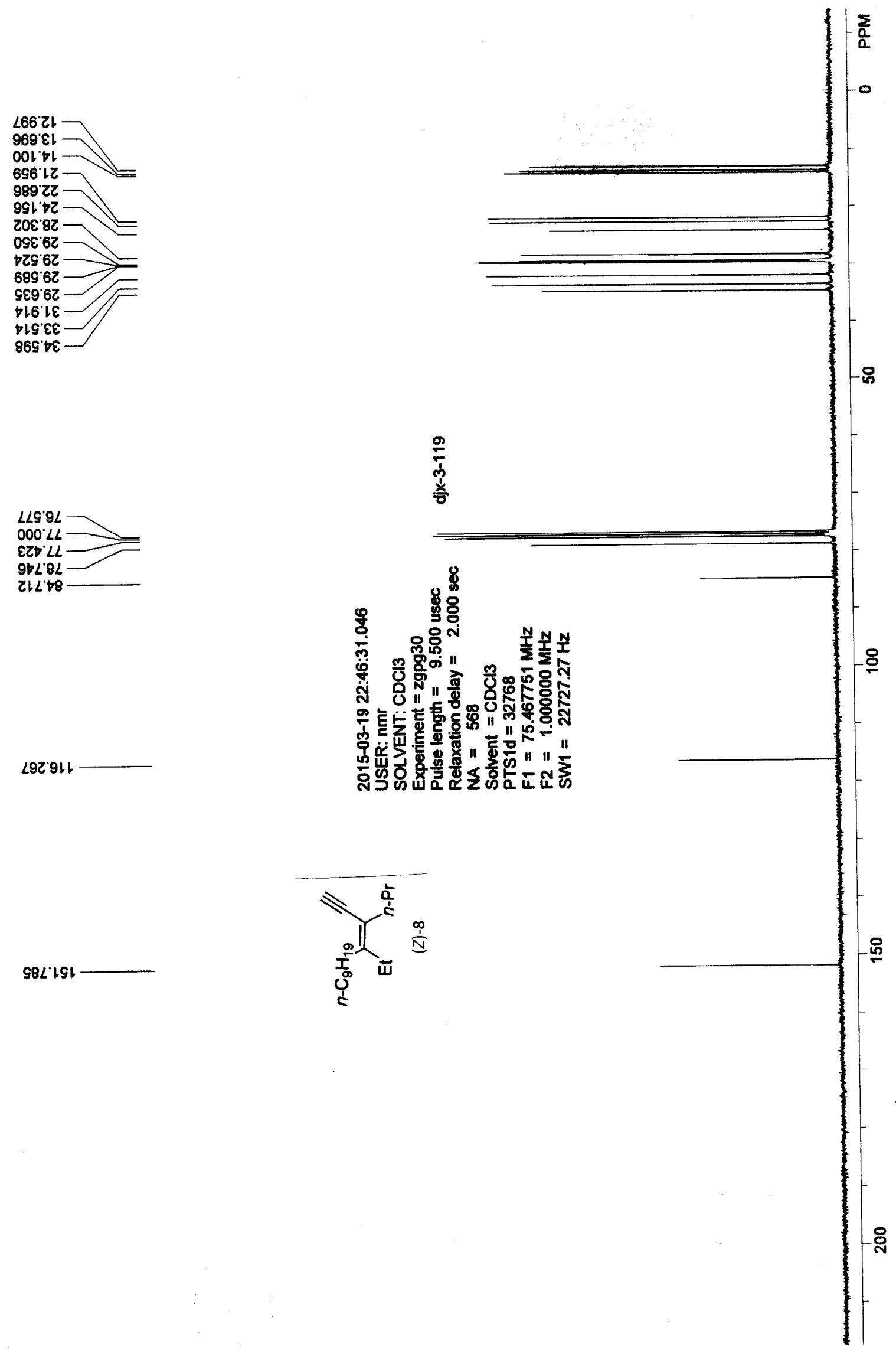

L9Z 9 LL

SRL'ISL

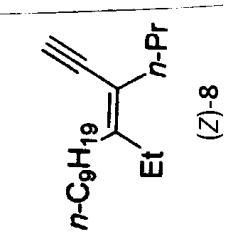



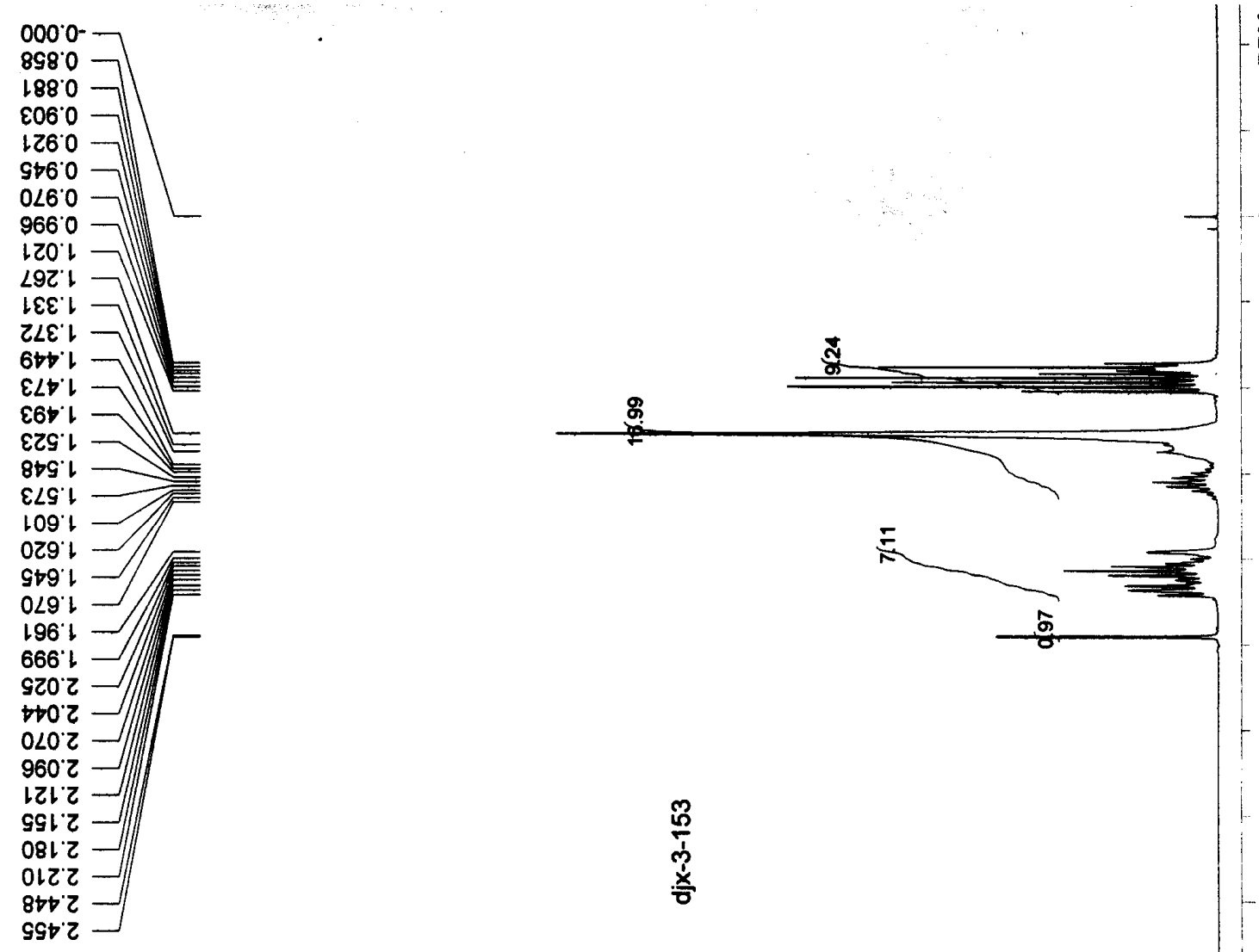

1629
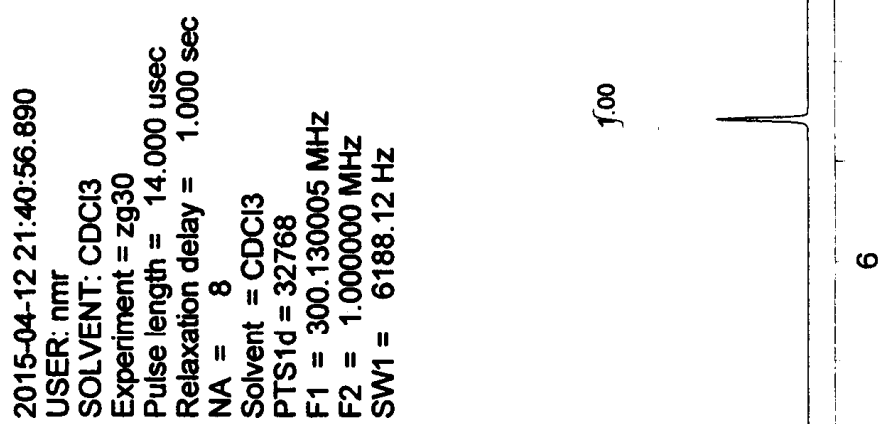

$8 L Z L$

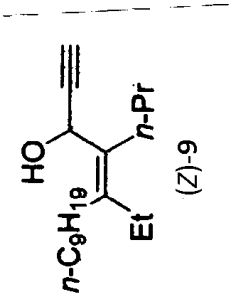


290 \&

909'

219.2Z

LZZ'bZ

\ZE'

$6 \checkmark 2 \cdot 62$

$09+62$

SIS $6 Z$

Etc. 62

$\checkmark \angle 8.62$

$9+908$

เ\&8'เย

198.19

cos. $\angle L S ' 9 L$

000.2

$\varepsilon Z \nabla^{\prime} L L$

Strit8

LOS' $|\varepsilon|$

๑ZEเレレ

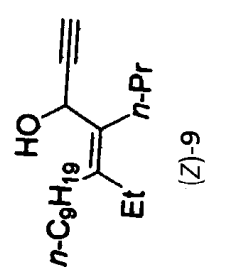

$\frac{m}{\frac{\pi}{0}}$
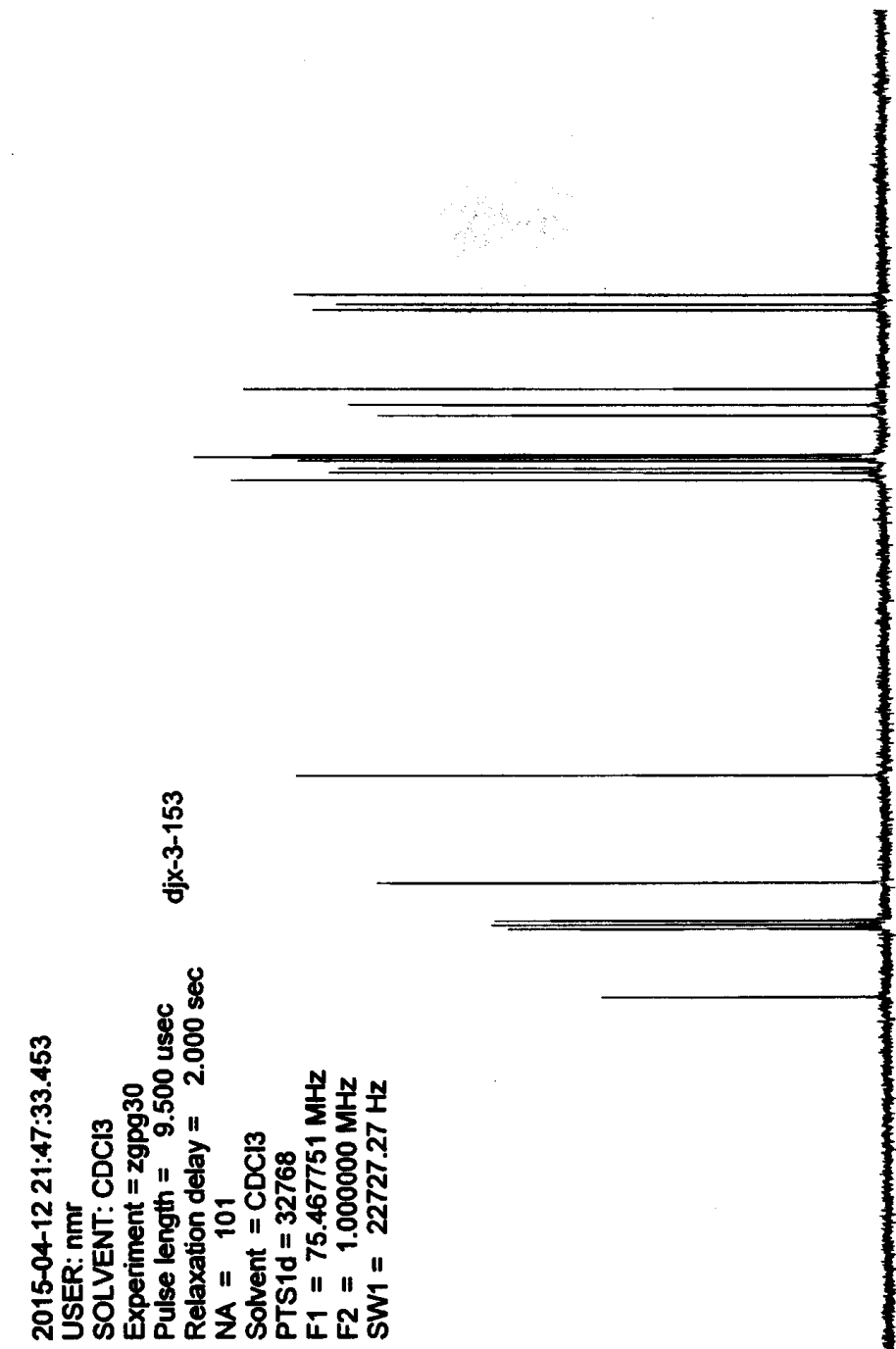

$-8$ 

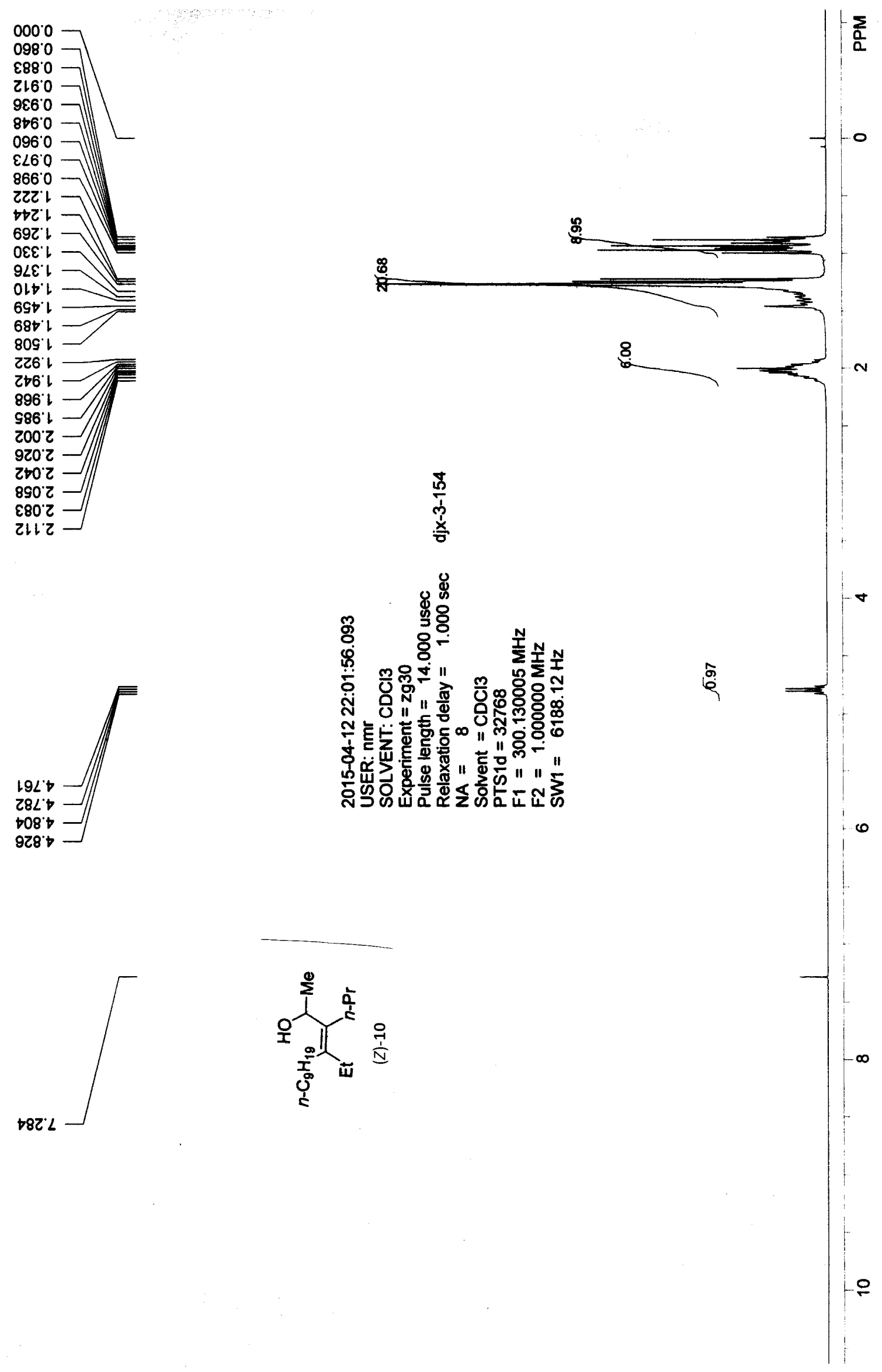

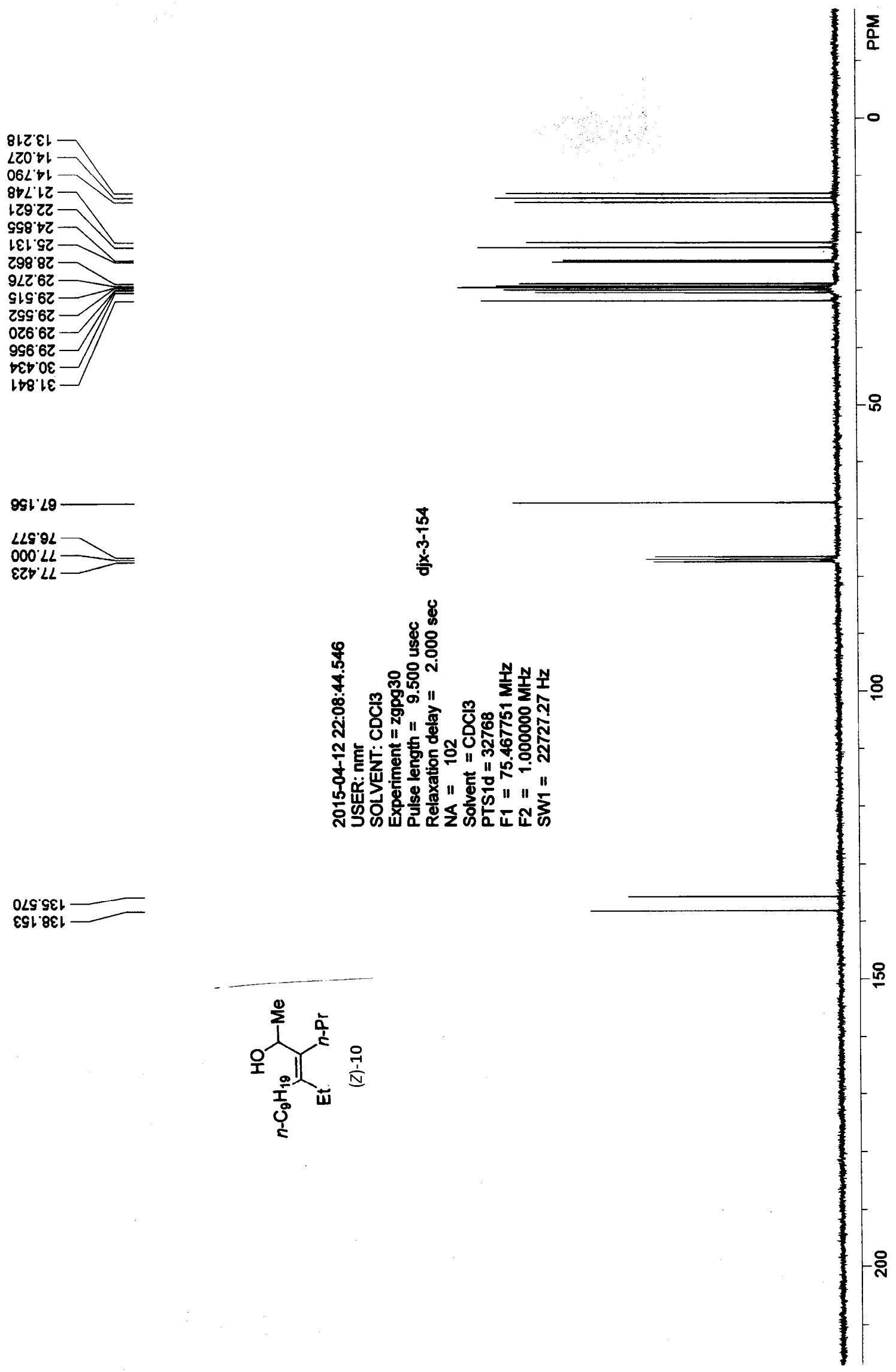

0L9' $9 \varepsilon$

ESL $8 E L$ 

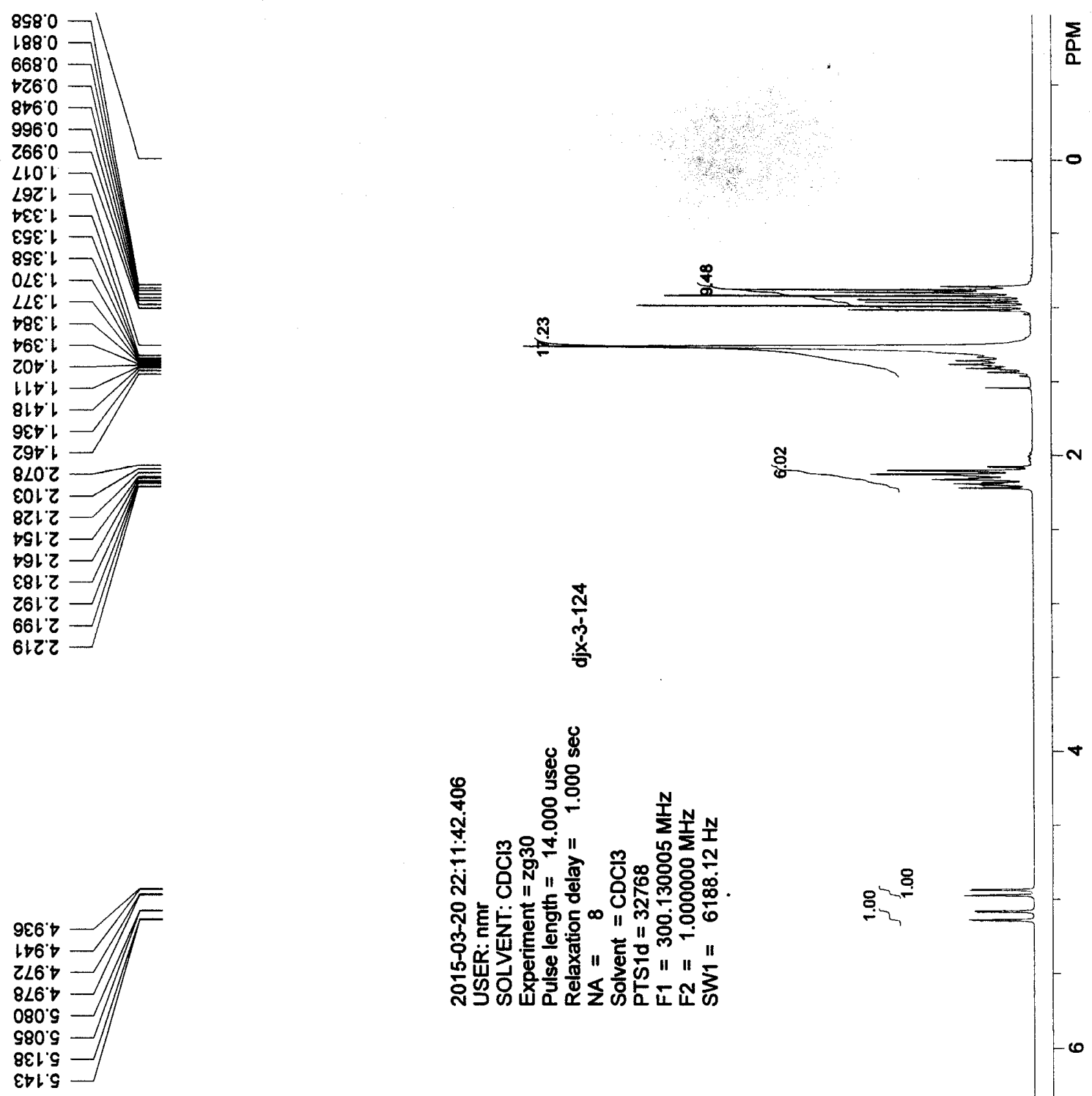

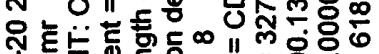

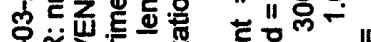

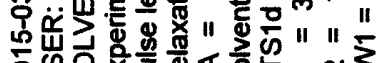

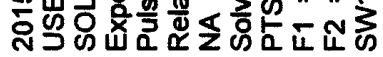
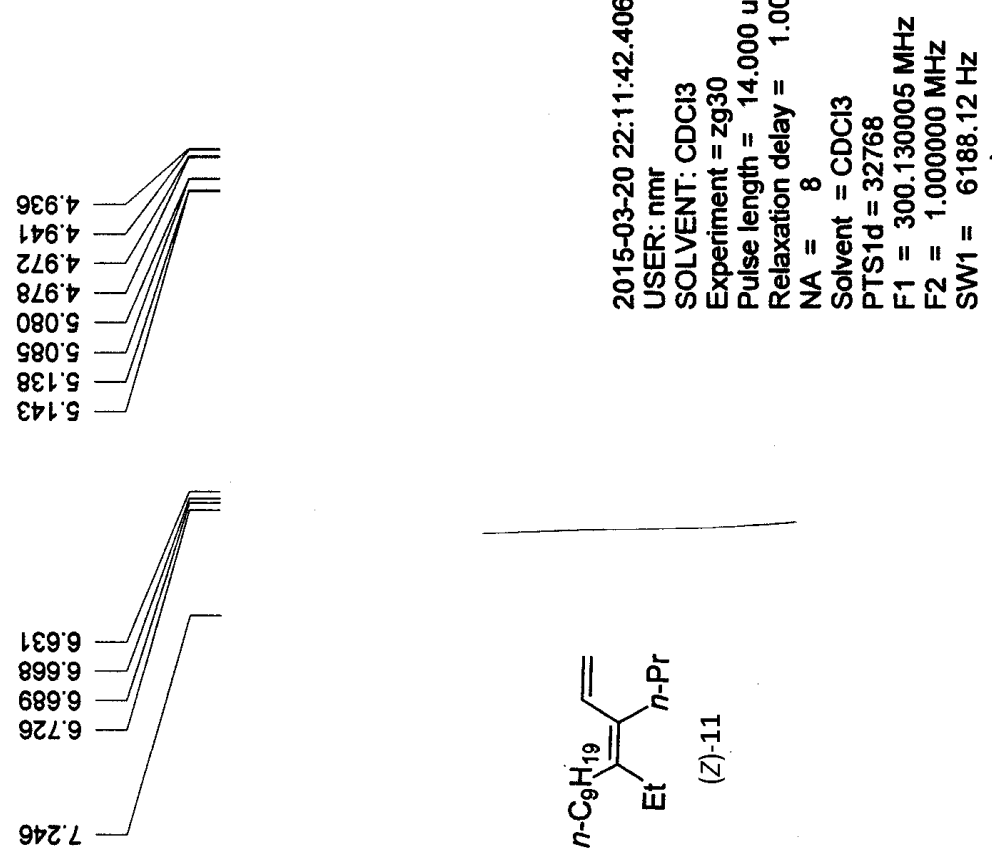

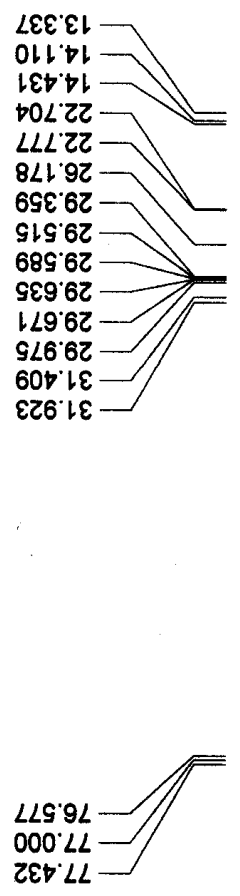

$606.0 \mathrm{LL}$
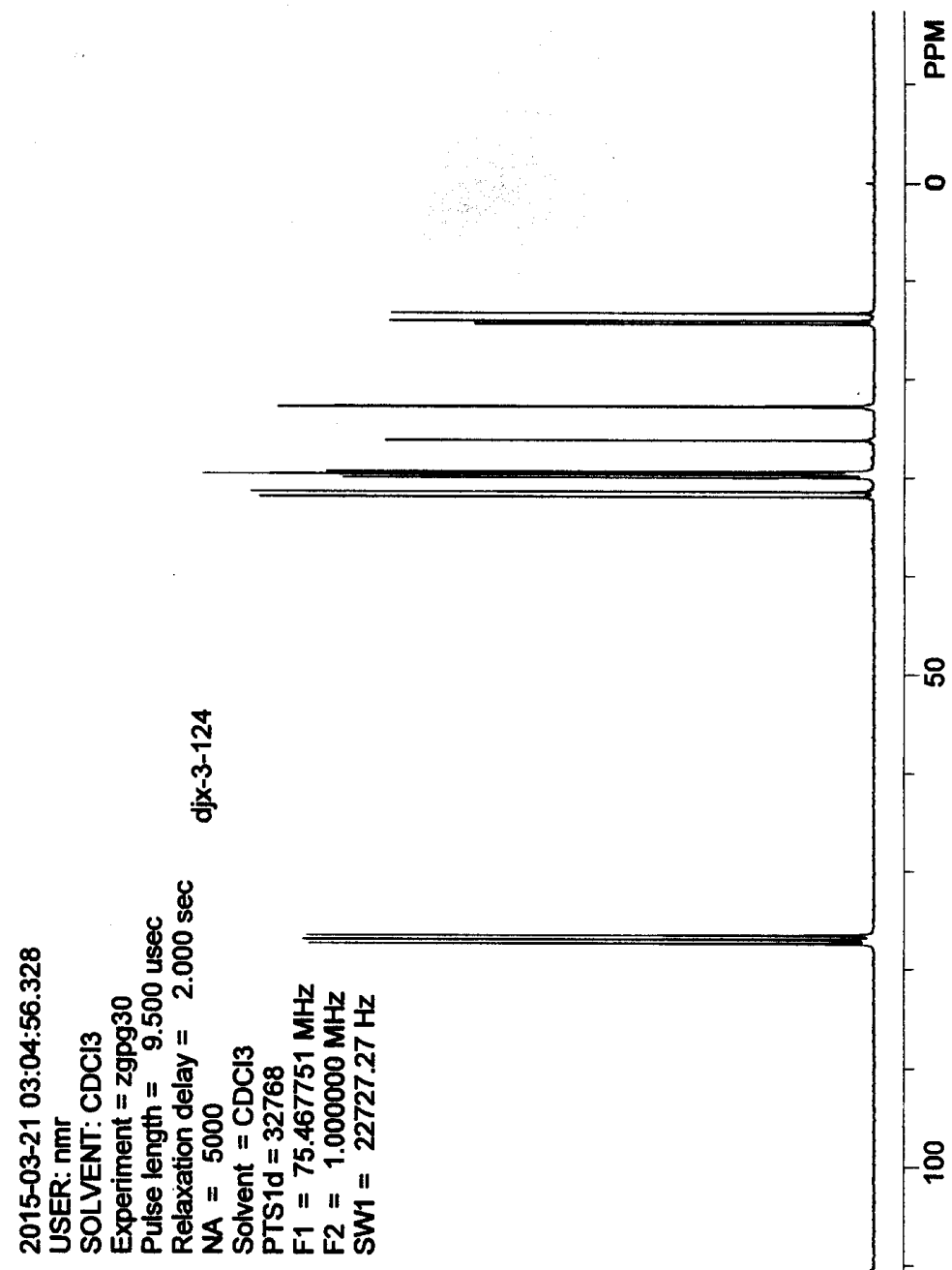

วะ乙 เยเ

68L' $D E L$

lal $2 t l$

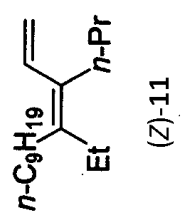

ホั
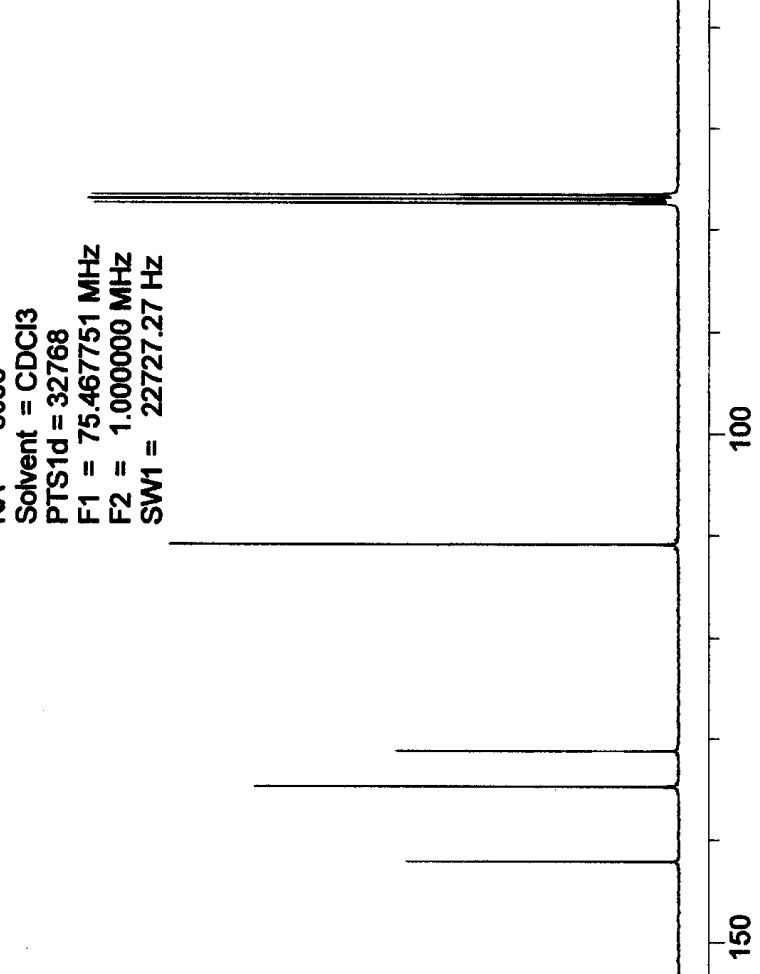

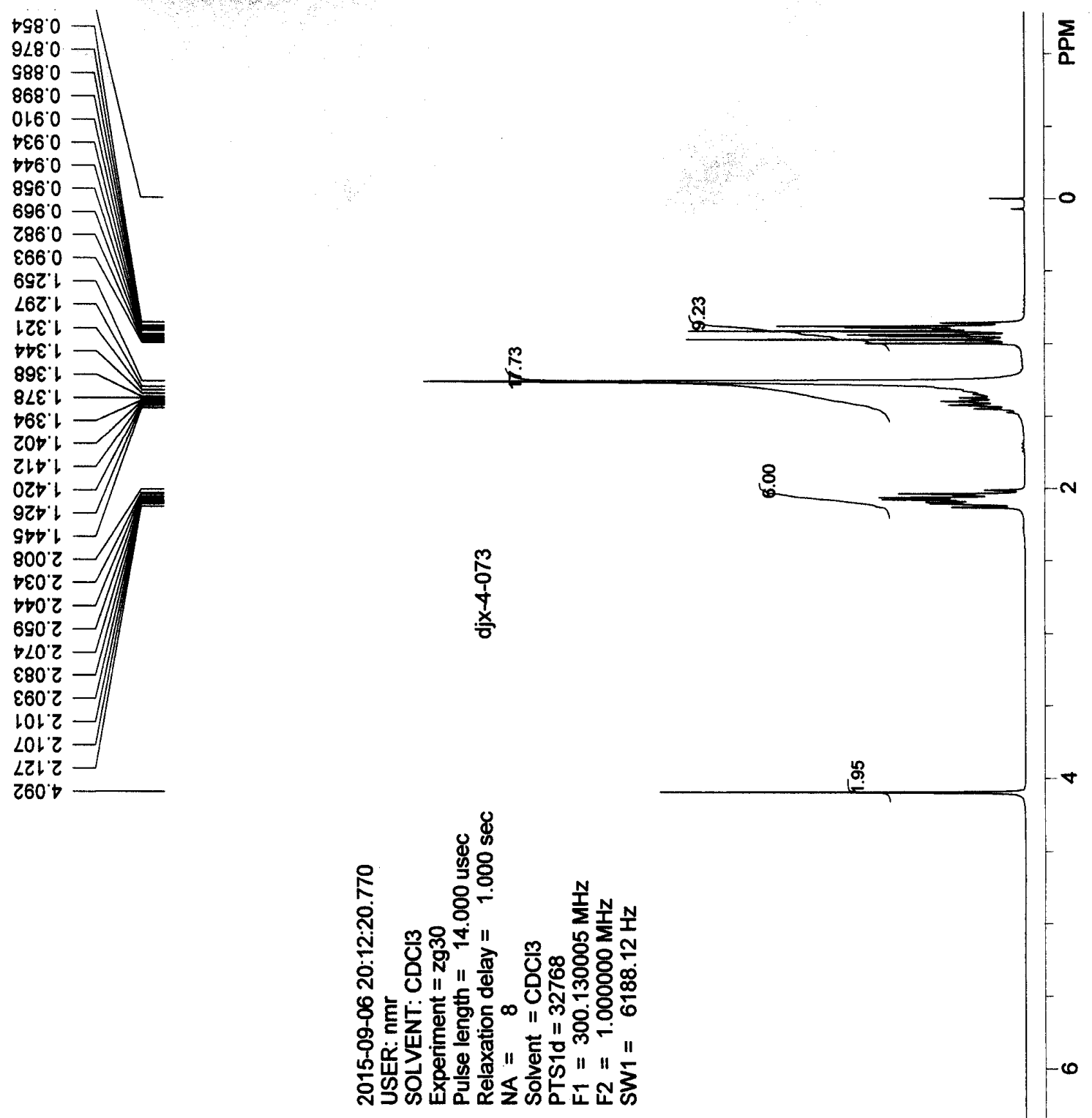

$Z \angle Z L$

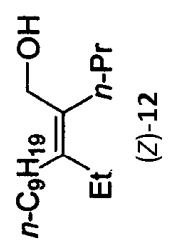



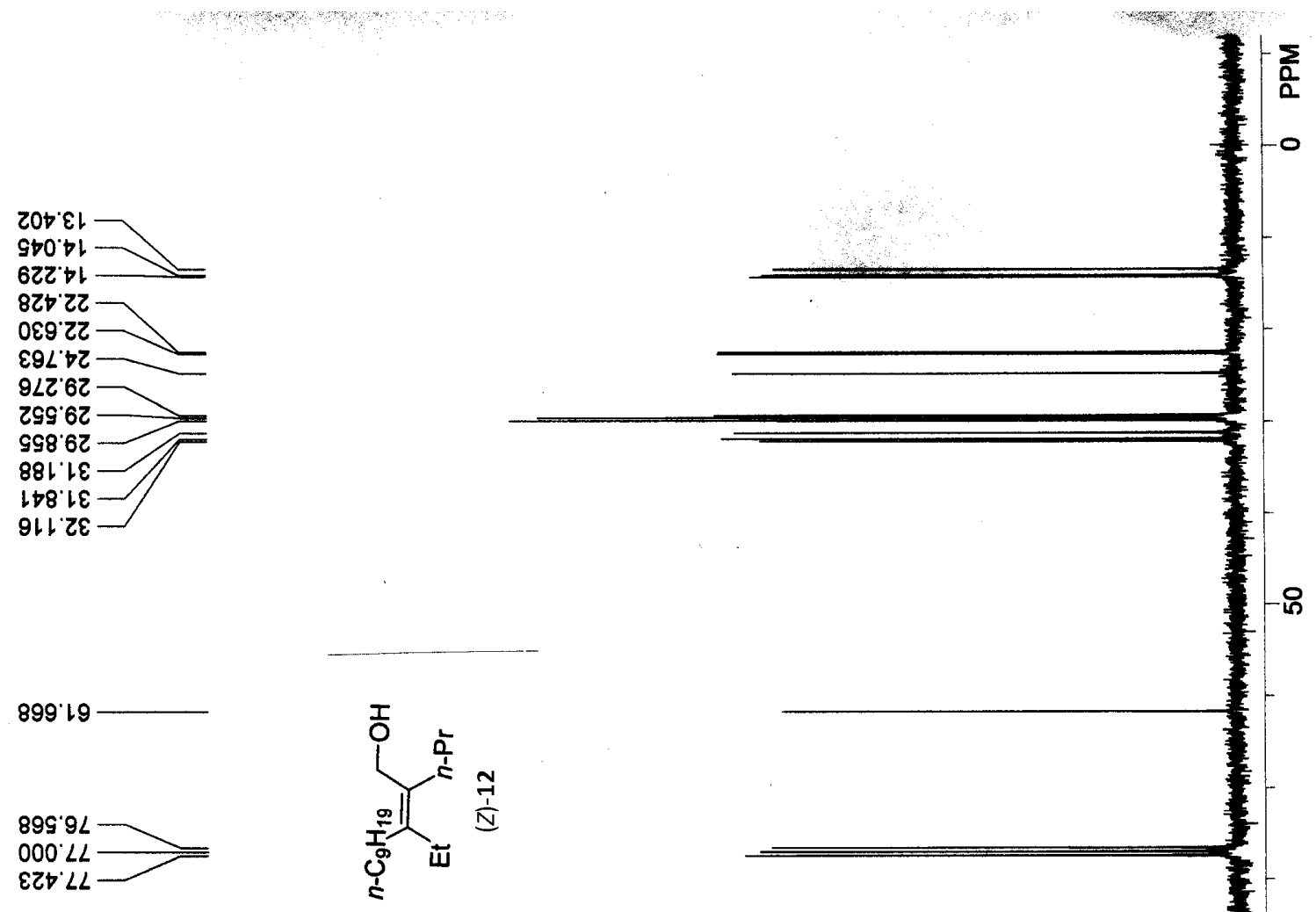

$\stackrel{2}{o}$
$o$
$\frac{1}{x}$
$\frac{x}{0}$

$86{ }^{\prime} Z \varepsilon$

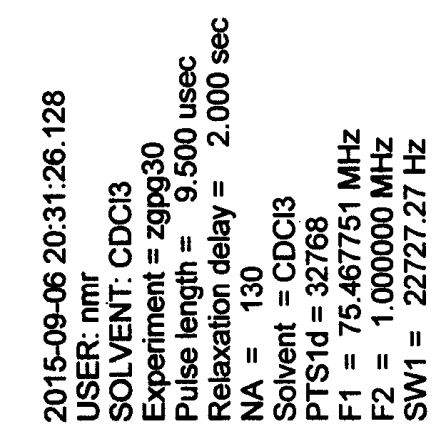

sE9 Ot 

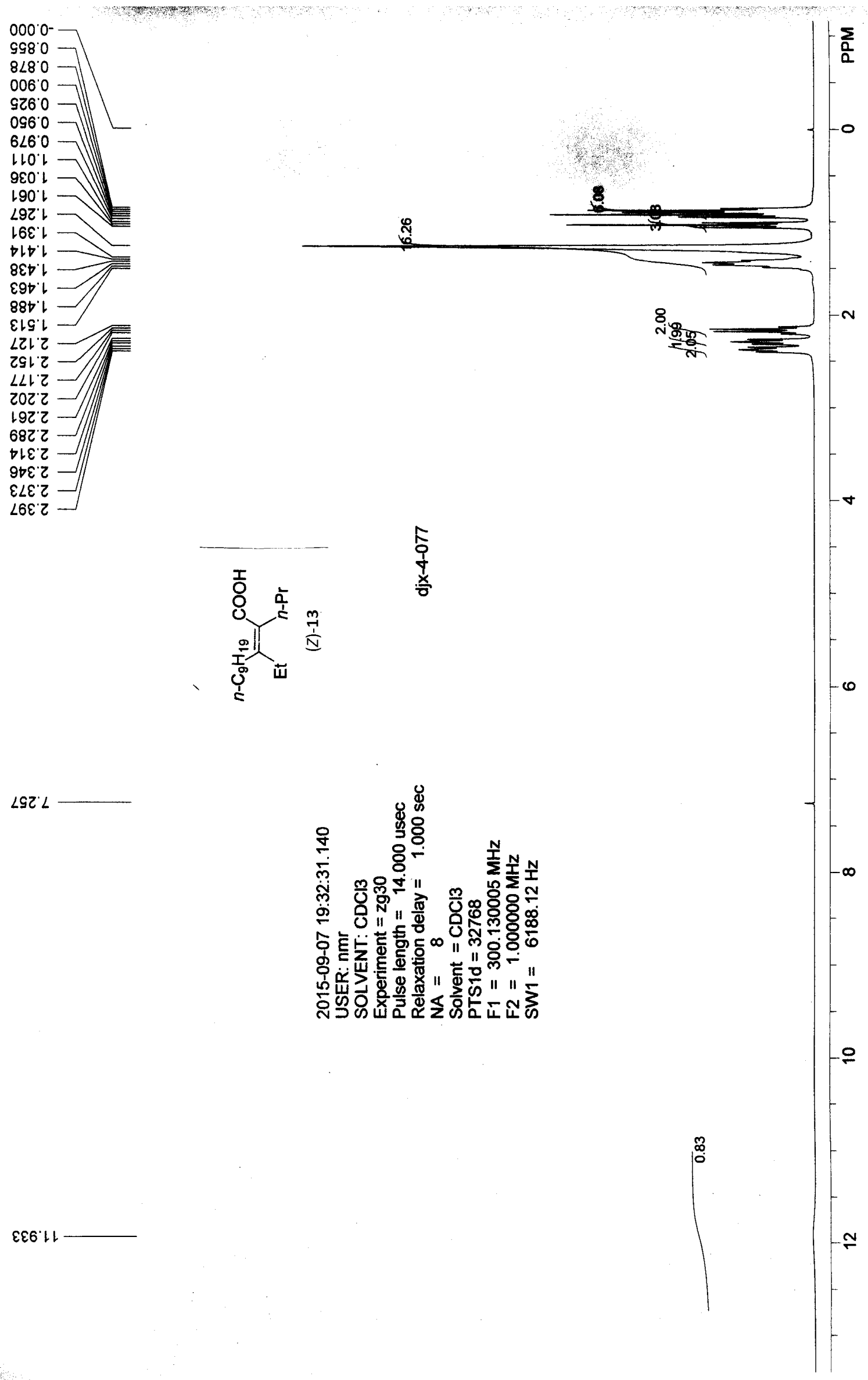

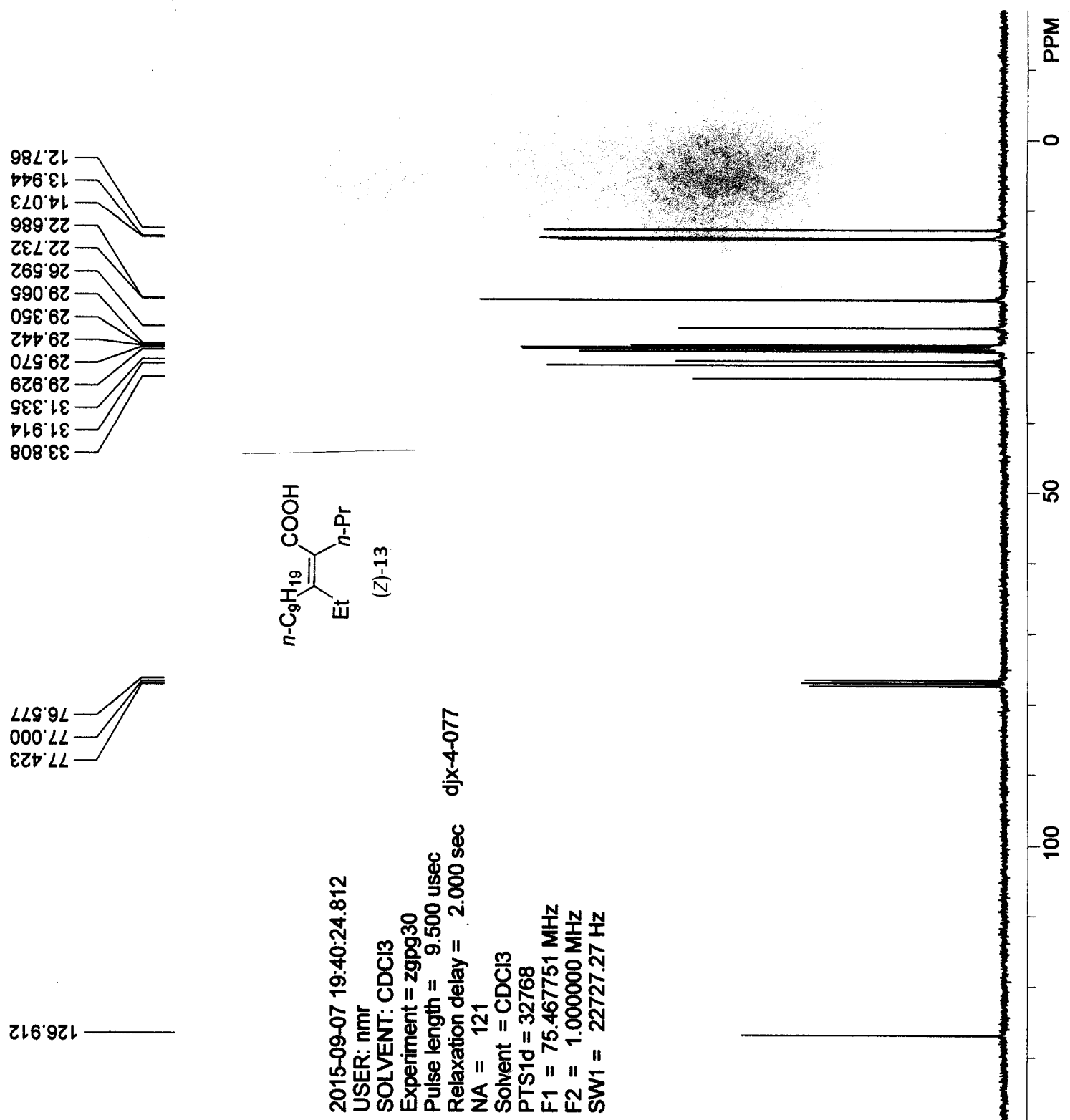

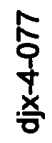

ZL6'9ZL

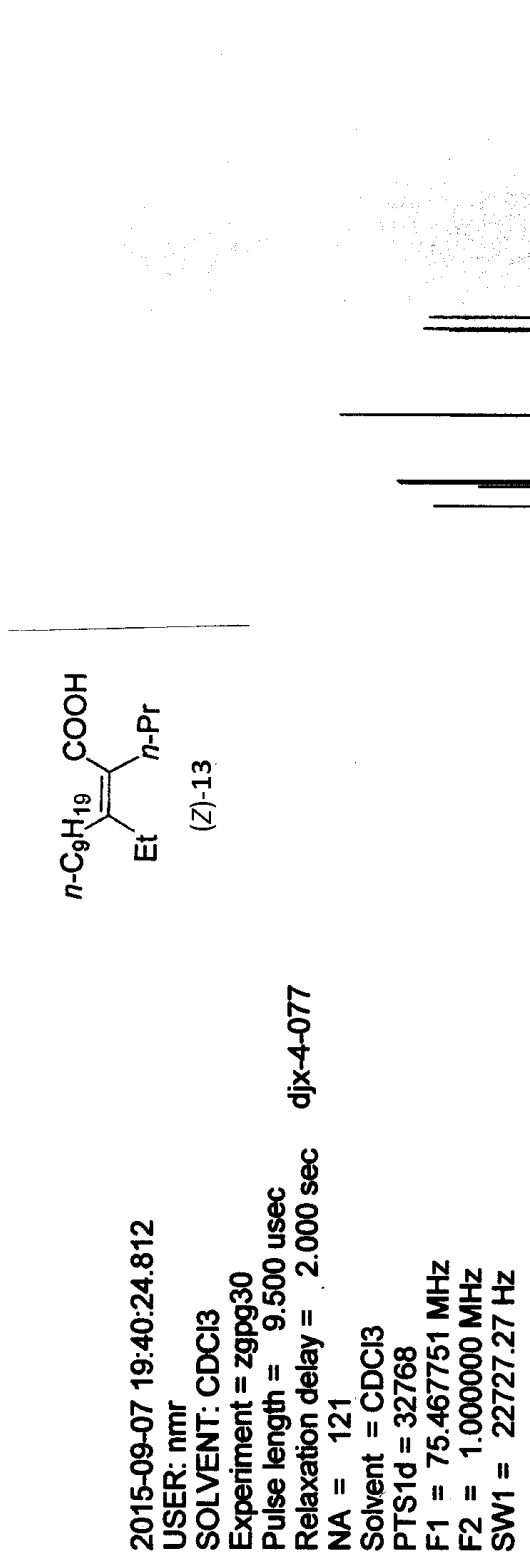

6ะ0'ง९

$968 ' 9 L b$

$\frac{2}{2}$ 